NBSIR 84-2982

\title{
Annual Report 1984 Center for Chemical Physics
}

P.J. Ausloos, R. A. Haines

U.S. DEFARTMENT OF COMMERCE

Netional Bureau of Standards

National Measurement Laboratory

Gaitinersburg, MD 2C899

\section{Ocroter 1384}

Issued December 1984

\section{Prepared for}

औS. DEPARTMENT OF COMMERCE ionai Bureau of Standards thersburg, MO 20899 



\section{ANNUAL REPORT 1984 \\ CENTER FOR CHEMICAL PHYSICS}

P.J. Ausloos, R.A. Haines

U.S. DEPARTMENT OF COMMERCE

National Bureau of Standards

Natioal Measurement Laboratory

Gaithersburg, MD 20899

October 1984

Issued December 1984

\section{Prepard for}

U.S. DEPARTMENT OF COMMERCE

National Bureau of Standards

Gaithersburg, MD 20899

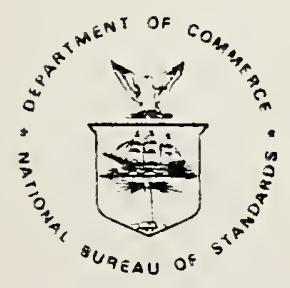

U.S. DEPARTMENT OF COMMERCE, Maicolm Baldrige, Secretary NATIONAL BUREAU OF STANDARDS, Ernest Ambler, Director 



\section{INTRODUCTION}

This book is a summary of the technical activities of the NBS Center for Chemical Physics (CCP) for the period October 1, 1983 to Seprember 30, 1984. The Center is one of four units in the National Measurement Laboratory. The Center for Chemical Physics (CCP), (1) develops and maintains the scientific competences and experimental facilities necessary to provide the nation with measurement methodologies and measurement services in the areas of thermochemistry, chemical kinetics, surface science and molecular spectroscopy; (2) measures, compiles, critically evaluates, and disseminates reference data to the industrial, government, and academic communities nationally and internationally; (3) develops theoretical methods and concepts, predictive equations and models; (4) develops standards and techniques of measurement; (5) provides federal, state and local governments, industry and the academic community with essential standards and certified standard reference materials needed to maintain the integrity of the measurement system; (6) provides consultative services and participates in collaboration efforts with other NBS Centers and the outside community.

The summary of activities is organized by division: Surface Science, Chemical Kinetics, Chemical Thermodynamics and Molecular Spectroscopy. Each division tells its own story and tells it in its own way. In general, there is an introduction that outlines the main concerns of the division, its goals and interactions. This is followed by a series of short reports on current research and future plans, of ten with explanations of why the work was undertaken. The publications during the year are listed.

A detailed table of contents has been provided. It will permit the reader to find those activities of greatest interest. More information about particular work may be desired. To obtain this, the reader should address the individual scientists or their division, c/o Center for Chemical Physics, Chemistry Building, B-i62, National Bureau of Standards, Gaithersburg, MD 20899. 
Certain commercial equipment, instruments, or materials are identified in this report to specify adequately the experimental procedure. Such identification does not imply recommendation or endorsement by the National Bureau of Standards, nor does it imply that the materials or equipment identified are necessarily the best available for the purpose. 
SURFACE SCIENCE - - DIVISION 541

1. Introduction

2. Surface Standards Program . . . . . . . . . . . . 1

A. Reference Data................ . . 4

B. Reference Materials . . . . . . . . . . 13

C. Review Articles and Books ............. 17

D. Standards-Committee Activities .......... 25

3. Surface Measurements Program ............ 30

A. Catalytic Surface Chemistry: Reaction Kinetics at High Pressures over Single Crystal Catalysts ........ . 30

B. Electron-Stimulated Desorption: Relation to Surface Molecular Structure and Reactivity ............. 32

C. Nucleation, Growth and Surface Atomic Structure of Ultrathin

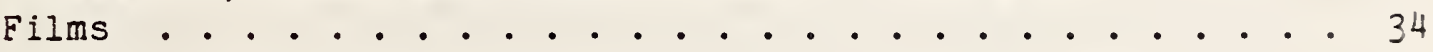

D. Electron Spectroscopy of Surfaces .......... 35

E. Ion-Surface Collisions and Atcmic Excitations ...... 35

F. Surface Theory . . . . . . . . . . . . 36

G. Fabrication of Fluorescent Screens . . . . . . . . 41

4. Surface Science Competence Program . . . . . . . . . . 42

A. Introduction ................ 42

B. Reports of Surface Science Competence Program Activities. . 42

C. Surface Science Experiments at the Brookhaven National Synchrotron Light Source............ . 58

5. Publications ............... . . 50

CHEMICAL KINETICS - - DIVISION 542

1. Introduction .................. i

2. Chemical Kinetics and Ion Kinetics Energetics Data Centers .. it 
A. Introduction . . . . . . . . . . . . . . . 74

B. Chemical Kinetics Data Center . . . . . . . . . . . . 74

C. Ion Kinetics and Energetics Data Center . . . . . . . . . 77

D. Future Plans . . . . . . . . . . . . . . . 78

3. Kinetics of Neutral Species . . . . . . . . . . . . . 82

A. Introduction . . . . . . . . . . . . . . . . 82

B. Gas Phase Kinetics . . . . . . . . . . . . . . 83

C. Condensed Phase Kinetics . . . . . . . . . . . . . 97

4. Ion Kinetics . . . . . . . . . . . . . . 103

A. Introduction . . . . . . . . . . . 103

B. Gas Phase Ion Kinetics ................. . 104

C. Liquid Phase Ion Kinetics . . . . . . . . . . . . 112

5. Publications ..................... . . 119

CHEMICAL THERMODYNAMICS - DIVISION 543

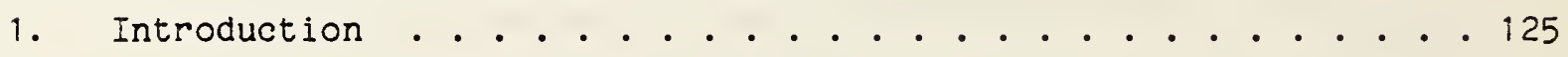

2. Chemical Thermodynamics Measurements . . . . . . . . . 135

A. Thermodynamics of Enzyme - Catalyzed Reactions . . . . . . 135

B. Weak Interactions in Model Enzyme - Substrate Systems . • . 138

C. Bomb Calorimetry of Key Biochemical Compounds . . . . . . 140

D. DSC Measurments and Standards . . . . . . . . . . . 141

E. Calorimetric Standards/OSRM . . . . . . . . . . . . . 141

F. Large Sample Combustion Flow Calorimetry . . . . . . . . 144

G. Drop Calorimetry of Non-Reacting Systems Below $2800 \mathrm{~K}$. . . 147

H. Raman Characterization of the Main Phase Transition in

Lipid Bilayers . . . . . . . . . . . . . . . 149

I. Euture Plans . . . . . . . . . . . . . . . . . 150 
3. Thermodynamic Data Compilations . . . . . . . . . . . 153

A. Chemical Thermodynamics Data Center . . . . . . . . . 153

B. Electrolyte Data Center . . . . . . . . . . . 157

C. Future Plans. . . . . . . . . . . . . . . . . 159

4. Publications . . . . . . . . . . . . . . . 161

MOLECULAR SPECTROSCOPY - - DIVISION 545

1. Introduction ... . . . . . . . . . . . . 165

2. High Resolution Spectroscopy Group . . . . . . . . . . . 168

A. Introduction . . . . . . . . . . . . . 169

B. Calibration Standards for the Infrared Spectrum . . . . . 174

C. Spectroscopy in Environmental and Space Science . . . . . . 179

D. Spectroscopy of Hydrogen-Bonded Systems and Other Clusters . . 182

E. Fourier Transform Spectroscopy . . . . . . . . . . 189

F. Development and Applications of Theories of Molecular Spectra 192

G. Microwave Spectra . . . . . . . . . . . . . 197

H. Molecular Spectra Data Center . . . . . . . . . . 198

I. Future Plans . . . . . . . . . . . . . . . 201

3. Laser Photochemistry Group . . . . . . . . . . . . . 201

A. Introduction . . . . . . . . . . . . . . . 201

B. Picosecond Studies of Molecular Energy Transfer . . . . . . . 201

C. Desorption of Molecules fron Surfaces . . . . . . . . . 205

D. Photochemistry in Molecular Beams . . . . . . . . . 205

E. Matrix Isclation Spectroscopy . . . . . . . . . . 208

E. Future Plans . . . . . . . . . . . . . . 212

4. Quantum Chemistry Group . . . . . . . . . . . . . . 216

A. Introduction . . . . . . . . . . . . . . 216 
B. Electronic Structure . . . . . . . . . . . . 216

c. Scattering . . . . . . . . . . . . . . . . 222

D. Future Plans . . . . . . . . . . . . . . . . 232

5. Publications . . . . . . . . . . . . . . 234 NBS Organization Chart

CCP Organization Chart

CCP Telephone Listing 


\author{
C. J. Powell, Chief \\ SUMMARY OF ACTIVITIES
}

Fiscal Year 1984

\title{
1. INTRODUCTION
}

\section{A. Background}

Over the last ten years, there has been a dramatic growth of surface science and its applications. At the national and local meetings of many professional societies, there are typically multiple sessions of papers concerned with the physical and chemical properties of surfaces. Similarly, many current professional journals and books are devoted in whole or in part to similar reports of recent advances in this field. These advances result from investigations of basic surface properties (such as composition, atomic structure, electronic structure) of surfaces and the dependence of many complex surface processes (such as molecular sticking, dissociation, diffusion, reaction, and desorption) on the basic properties and other variables relevant to catalysis, corrosion, thin-film systems, microelectronics, computer elements, and communications applications. Investigations of this type range from fundamental studies, at an atomic and molecular level, on clean single-crystal surfaces to similar studies on appropriately modified surfaces or structures and lead not only to the development of surface science but also to new materials, devices, and processes. Similar investigations, principally measurements of surface composition and of other needed properties, are frequently made of a large variety of materials (e.g., metals, polymers, semiconductors, oxides, glasses, etc.) after fabrication and at various time during their service life for quality control, process optimization, and failure analysis.

A large number of techniques are utilized for surface characterization. A choice of one or more techniques is made typically on the basis of the property to be measured, the specimen material, and the specific needs (e.g., spatial resolution, chemical information, sensitivity, degree of difficulty, and cost). The most common type of surface measurement is the determination of surface composition. Most surface analyses now are quaittative but there is a growing demand for quantitative analyses with improved accuracy. At the present itme, there are very few standard procedures and iimited reference data and reference materials. A variety of techniques is employed for the determination of surface atomic structures, surface electronic structure, and for investigations of surface processes such as reactions, diffusion, segregation, etc. Theories of the measurement process need to be developed for the newer techniques. For all techriques, there is a need 
for relevant procedures, tests to establish measurement reliability, and applicable reference data. In most cases, the measurement science needs to be developed since knowledge of the key concepts and parameters is often extremely limited.

The techniques utilized in surface science have generally been applied to characterize surfaces in an ultrahigh vacuum environment. The solution of many scientific and technical problems often requires removal of Iiquid- or gas-phase material prior to surface examination and/or the removal of solid-phase material to expose a region of interest. While this approach has often been successful, artifacts of many different types can arise during the removal of one or more phases. There is therefore a growing need for improved measurement methods that can be used for the in situ characterization of solid-solid, solid-liquid and solid-gas

interfaces with the sensitivity, elemental specificity, and spatial resolution comparable to those attained with the current surface-characterization methods.

\section{B. Goal, Objectives and Division Program}

The goal of the Surface Science Division is to improve the quality of existing surface-characterization measurements and to extend the present capability. Specific objectives of the Division are:

(1) Perform experimental and theoretical research in surface science to provide a scientific base for surpace-measurement methodology;

(2) Develop measurement methods and provide properties data for the physical and chemical characterization of surfaces and surface processes to meet identified needs of industry, government, and other groups;

(3) Develop standard procedures, calibration data, and reference materials in cooperation with national and international standards organizations; and

(4) Develop expertise in selected new areas of science in order that NBS can provide measurement services where needs are anticipated in the near future.

Objectives (1) through (3) are met organizationally through an NBS task titled "Development of Measurement Methods for Surface Properties" (Task 15502). The Division's program for this task is divided into two principal activities, a Surface Standards Program and a Surface Measurements Program. Work performed during the year in these two areas is reported in sections 2 and 3, respectively. This work is supported by NBS resources (including some funds transferred through the office of Standard Reference Data) and by contracts with three Federal agencies (Office of Naval Research, Department of Energy, and National Aeronautics and Space Administration). 
Objective (4) is met as a result of the NBS Competence Program through a task titled "Surface Science" (Task 26103). Some objectives of this task are also supported by contracts with other agencies (Office of Naval Research, Department of Energy). A separate report on these activities is given in Section 4.

\section{Technical Output and Professional Interactions}

Sections 5 and 6 of this report contain listings of the publications and talks, respectively, for the past year by Surface Science Division staff and for projects supported by the Division. These Iistings were prepared before the end of the fiscal year and include publications and talks for the latter part of FY 83 not given in the previous Division report.

Many members of the Division staff hold leadership positions in professional societies and groups, as listed in Section 8 . These interactions not only reflect the professional esteem in which these stafe members are held but provide useful mechanisms for both disseminating the results of NBS work and. for learning of significant measurement problems being faced by those using surface-characterization equipment.

Interest in surface science and surface characterization at NBS continues to grow. The Division has had an active Seminar program, as indicated in Section 7 , and these seminars have been attended by other NBS staff. The Division staff also participates in an informal weekly lunch meeting, attended by scientists from a number of NBS organizational units, at which developments in surface science are discussed. Members of the Division staff are frequently consulted on a wide range of NBS problems.

Much of the Division's work is carried out in collaboration with other scientists within NBS, within the U.S.A., and internationally. The nature of these collaborations is shown in Section 9 . These collaborations are considered important because they enable a relatively small Division staff to work synergistically with other experts, they enable us to have access to unique facilities or expertise, and they enable our expertise to be extended to a larger range of important scientific and technical problems.

The Division has been fortunate in having eleven visiting scientists work at NBS for varying periods during the past year, as indicated in Section 10. These scientists enrich our staff and our project3. A further enrichment of our programs comes erom Postdoctoral Research Associates who hold 1 - to 2-year appointments; six Postdcctoral Research Associates worked in the Division during FY 1984. 


\section{SURFACE STANDARDS PROGRAM}

The term "surface standards" is intended to cover a broad range of activities that are directed to improving the quality of surface-characterization measurements through: (1) development of needed reference data and standard reference materials; (2) leadership in standards-committee activities, the development of standard practices, and other actions to improve the accuracy and reliability of surface-analysis measurements; and (3) publication of review articles and books that enable scientists to make surface-characterization measurements of the needed quality.

\section{A. Reference Data}

We describe here results of projects in which needed reference data are being generated. Section 2.C includes recent review articles that summarize and evaluate data required in a number of surface spectroscopies.

\section{Measurement of Absolute Ion Sputtering Yields} (J. Fine)

The principal aim of this work is to obtain absolute sputtering yields of $1-5 \mathrm{keV}$ ions on well-characterized elemental samples in order to improve the accuracy of depth-profiling measurements by current surfacemethods. The accuracy of depth-profiling measurements is at present limited by the unavailability of accurate, reliable, sputtering-yield data and by the lack of knowledge of the topographical and morphological effects produced by ion bombardment.

The use of quartz-oscillator techniques to measure mass loss due to ion bombardment can result in very accurate determinations of ion-sputtering yields. In addition to the third-harmonic oscillator method deveioped at NBS, another system (operating in the fundamental mode) has been developed at the $J$. Stefan Institute in Ljubljana, Yugoslavia as part of a joint US/Yugoslavia program and is presently in operation.

Measurements have been made using the quartz oscillator facilities in Ljubljana to determine sputtering yields of $\mathrm{Ni} / \mathrm{Cr}$ multilayered thin-film materials of structure and morphology similar to those of the NBS $\mathrm{Ni} / \mathrm{Cr}$ SRMS (Section 2.B). Similar measurements have been initiated at NBS using the third-harmonic method. Quartz crystal of our own design, having highly polished surfaces, were used as substrates during the actual production-run deposition of our SRM structures. These same SRM materials will be used for mass-loss sputtering-yield determinations; such data will then permit cur $\mathrm{Ni} / \mathrm{Cr}$ SRMs to be useful in the calibration of depth scales for other materials whose sputtering yields are known.

Similar measurements are planned on a variety of in-situ prepared elemental targets to obtain ion-cose dependent sputtering yields. 
Sputtering techniques for depth-profile analysis are presently being used in conjunction with a number of surface analytical methods to analyze compositional changes of materials at surfaces and at interfaces. Many of these techniques have now progressed to the point of being able to provide quantitative sputter-depth-profile information yet the necessary sputtering yield data is not readily available in easy-to-use, evaluated rorm.

We therefore will prepare a compilation of evaluated sputtering-yield data for those parameters that will have specific use in surface analysis and depth profiling. This work is supported by the NBS office of Standard Reference Data. Our compilation will be in tabular form for those ion species ( $\mathrm{Ne}, \mathrm{Ar}, \mathrm{Kr}, \mathrm{Xe}, \mathrm{O}_{2}, \mathrm{~N}_{2}, \mathrm{Cs}$ ) and energies $(0.5$ to $20 \mathrm{keV}$ ) and angles of incidence used in surface characterization. We will initially prepare such compilations of sputtering yields for elemental targets only. we anticipate making use of the HP1000 Data System in the Center for Chemical Physics. are:

This project was initiated in June, 1984. Progress to date and plans

a. A system designed to compile sputtering yield data is being developed and is to be implemented on the HP1000 System.

b. A bibliography of sputtering yield data is being assembled and reprints are being collected.

In the coming year we intend to abstract, evaluate, and compile sputtering-yield data of interest to the surface-analysis and sputterdepth-profiling community on elemental targets.

3. Measurement of Core-Level Binding Energies

(C. J. Powell and N. E. Erickson)

Accurate reference values of the binding energies of core-level electrons are needed for the commoniy used technique of $x$-ray photoelectron spectroscopy (XPS) and for other surface-characterization methods. These values are needed for the calibration of instrumental energy scales. For this application, data are required for a limited number of pure materials that adequately cover the range of common measurements.

We have previously made high-accuracy measurements of the principal core-level binding energies of $\mathrm{Ni}, \mathrm{Cu}, \mathrm{Ag}$, and $\mathrm{Au}$ by XPS. These values compare favorably with similar high-accuracy values measured independently in other laboratories by other methods. We have similarly made high-accuracy measurements of the kinetic energies of Auger electrons irom $\mathrm{Ni}, \mathrm{Cu}, \mathrm{AE}$, and $\mathrm{Au}$. 
At the time that these measurements were being made, we noticed that literature values of XPS binding energies appeared to be systematically higher, by about $1 \mathrm{eV}$, than those measured by appearance-potential spectroscopy (APS). As systematic errors can occur and remain undetected in binding-energy measurements by any one technique, we have measured binding energies by XPS, APS, and core-level electron energy-loss spectroscopy (EELS) with the same samples and the same reference power supplies to eliminate the more obvious sources of possible error in comparisons of measurements made in different laboratories.

We have determined the $\mathrm{L}_{3}$ binding energies for $\mathrm{Ti}, \mathrm{V}, \mathrm{Cr}, \mathrm{Fe}$, $\mathrm{Co}$, and $\mathrm{Ni}$ by XPS, APS, and EELS. XPS measurements were made from the energy positions of the photoelectron peaks and the EELS measurements were made of the minimum energy loss for a particular core excitation. The latter measurements were made as a function of incident electron energy, from about $50 \mathrm{eV}$ above the threshold for $\mathrm{L}_{3}$-shell excitation to $1500 \mathrm{eV}$; in general, the measured EELS binding energies varied slightly with incident energy. The determination of an APS binding energy requires comparison of the measured spectrum with a calculated spectrum. The latter is based on a one-electron model in which the core-electron and the scattered electron both reach final states above the Fermi level. If agreement of the calculated and measured APS data is obtained, it is then possible to identify the electron energy on the experimental spectrum that corresponds to a final state with two electrons at the Fermi level.

Table 1 summarizes our numerical results. For $\mathrm{Ti}$ and $\mathrm{V}$, there was significant disagreement in the calculated and measured APS spectra so the indicated APS binding energies must be regarded only as estimates. For $\mathrm{Cr}, \mathrm{Fe}, \mathrm{Co}$, and $\mathrm{Ni}$, the differences between XPS and APS binding energies range between 0.6 and $1.5 \mathrm{eV}$, a result similar (for $\mathrm{Fe}, \mathrm{Co}$, and $\mathrm{Ni}$ ) though different in detail to that found in the literature. In general, the EELS energies do not interpolate between the APS and XPS binding energies, the limits expected for adiabatic and sudden excitation, respectively.

Our principal conciusions are as follows: (1) There is a breakdown of the simple one-electron model to describe the APS data for $\mathrm{Ti}$ and $\mathrm{V}$. (2) There may be different final states for each type of excitation. We do, in fact, observe changes in EELS Iineshapes for $\mathrm{Ti}, \mathrm{V}$, and $\mathrm{Cr}$, as the incident energy is varied. (3) There appears to be a varying screening response of the solid for each type of excitation. (4) Our results indicate that conventionally measured binding energies obtained by different methods may differ. A more detailed knowledge of the applicable final states and selection rules is needed to transfer binding energies from one spectroscopy to another.

We plan to compare our present method of calibrating electron energies using a thermionic electron source with a field-emission electron source. The latter method may be more accurate since no work-function correction is required. We also plan to use an existing $x$-ray monochromator as the detection device in a bremsstranlung isochromat spectrometer. Core-level binding-energy measurements by this technique will complement the current measurements with XPS, APS, and EELS. 
Table 1. Summary of $\mathrm{L}_{3}$-shell binding-energy data for $3 \mathrm{~d}$ elements obtained by APS, EELS, and XPS. The EELS binding energies were found to depend on incident electron energy; the lowest and highest measured EELS binding energies are shown here and correspond to the lowest and highest incident energies, respectively, used for each element. The uncertainty of each measurement is estimated to be $\pm 0.2 \mathrm{eV}$. The APS data for $\mathrm{Ti}$ and $\mathrm{V}$ are questionable as there was inadequate agreement of measured and calculated spectra.

Element

APS

Binding energy ( $\mathrm{eV}$ )

EELS

XPS

$\begin{array}{llll}\text { T1 } & 455.1(?) & 453.5-454.0 & 454.1 \\ \mathrm{~V} & 510.9(?) & 512.0-512.4 & 512.6 \\ \mathrm{Cr} & 573.9 & 573.7-574.5 & 574.6 \\ \mathrm{Fe} & 705.8 & 706.7-707.0 & 707.3 \\ \mathrm{Co} & 777.7 & 777.7-777.9 & 778.7 \\ \mathrm{Ni} & 852.2 & 851.6-851.8 & 852.8\end{array}$

4. Reference Data for X-ray Photoelectron Spectroscopy and Auger-Electron Spectroscopy

(C. J. Powell and N. E. Erickson)

A project was initiated in FY 82 with funding by the NBS office of Standard Reference Data to prepare compilations and evaluations of core-electron binding energies and of the kinetic energies of selected Auger electrons for a wide range of elements and compounds. In particular, data are being compiled of the "chemical shifts" that can give userul information on the chemical state of a particular element. Data of this type are now scattered throughout the scientific literature but there is a need both for a convenient single source and for evaluation of the sometimes conflicting data. The reference data are being compiled Dr. C. D. Wagner, a contractor to NBS, and the Surface Science Division is providing technical review. Dr. Wagner has currently zenerated over 8000 "lines" of data in his data files. It is expected that a report will be prepared for the Journal of Physical and Chemical Reference Data.

5. Inelastic Mean Free Paths and Attenuation Lengths of Electrons in Solids

(c. J. Powell)

The inelastic mean free path (IMFP) of low-energy electrons in solids is a key parameter in quantitative surface analysis by Augerelectron spectroscopy (AES) and $x$-ray photoelectron spectroscopy (XPS). For electrons of energy between 100 and $2000 \mathrm{eV}$, the range of practical 
interest for AES and XPS, the IMFP is typically between 3 and about $30 \AA$. Experimental measurements of the related quantity, the attenuation length (AL), are difficult and most published AL values are believed to have substantial uncertainties. (The IMFP is the average distance between successive inelastic collisions while the AL results from measurements analyzed with a model in which elastic electron scattering is assumed to be insignificant.) Theoretical IMFP calculations may also be in error on account of approximations and assumptions made in the analysis. It has therefore been difficult to reconcile available IMFP calculations and AL measurements and, in particular, to establish how IMFP and AL values vary with material and electron energy.

IMFP calculations have been made for eight materials ( $C, M g, A l$, $\mathrm{Al}_{2} \mathrm{O}_{3}, \mathrm{Cu}, \mathrm{Ag}, \mathrm{Au}$, and $\mathrm{Bi}$ ) from tabulations of dielectric data. The dielectric data, from the infrared to the $x$-ray region, satisfies various sum rules. As assumption needs to be made concerning the momentum-transfer dependence of the complex dielectric constant but this choice does not affect the conclusions appreciably.

Previous authors have fitted values of IMFP, $\lambda$, to a relation of the form $\lambda \propto E^{\mathrm{n}}$, where $E$ is the electron energy. A widely-used empirical formula based on AL data suggests that $n=0.5$ while simple theory indicates $n=0.75$. The present calculations show that $n=0.75$ for the "free-electron-like" solids (e.g., Mg, A2, Bi) but that $n=0.6$ for the noble metals. These differences, which are in accord with trends of the available AL data, can be explained by different spectral distributions in the dielectric data. Differences in the absolute values of IMEP from one material to another (for a constant electron energy) appear to be greater than expected from the empirical formula. These results indicate that additional IMFP calculations and more AL data are needed in order to perform quantitative surface analyses by AES and XPS with the desired accuracy.

6. Electron Attenuation Lengths in Condensed Molecular Films (R. L. Kurtz, R. Stcckbauer, N. Usuki, T. E. Madey)

Measurements of the electron attenuation length, $\lambda$, in condensed molecular layers have been made for electron energies from $18-70 \mathrm{eV}$ at the NBS SURF-II synchrotron radiation facility. The molecules $\left(\mathrm{H}_{2} \mathrm{O}\right.$, $\mathrm{CH}_{3} \mathrm{OH}$, and $\mathrm{C}_{5} \mathrm{H}_{12}$ ) were condensed with unit sticking probability at $90 \mathrm{~K}$ on clean $\mathrm{Cu}(100)$ by dosing with the known flux of a calibrated micro-capillary array doser. The drop in photoelectron emission intensity from the $\mathrm{Cu} 3 \mathrm{~d}$ band was measured as a runction of increasing layer thickness. By varying the incident photon energy, the resultant photoelectron kinetic energy was varied. Semi-logarithmic plots of the emission intensity versus the molecular dose yield straight lines whose inverse slopes give the attenuation length directly. Plots of $\lambda$ vs. electron energy for each molecule exhibit weak energy dependences, wi th broad minima in the range 30-40 eV. The mean minimum values of $\lambda$ for each film are: $C_{6} \mathrm{H}_{6},(6.1 \pm$ 1.5) $\AA$; $\mathrm{CH}_{3} \mathrm{OH},(7.3 \pm 1.8) \AA ; \mathrm{H}_{2} \mathrm{O},(9.0 \pm 2.2) \AA$. These are the first 
such measurements for these molecules in this energy range, and have important implications for the theory of electron transport in solics, as well as radiation damage processes in biomaterials.

7. Angular-Anisotropy Effect in Measurements of Electron Attenuation Lengths in Crystaliine Films

(i. E. Egelhoff)

Attenuation lengths of low-energy electrons in solids, important for quantitative surface analysis by Auger-electron spectroscopy (AES) and $x$-ray phctoelectron spectroscopy (XPS), have been frequently measured from the characteristic decay or growth of an AES and XPS signal as a film of increasing thickness is deposited on a substrate (see section 2.i(f)). Te have recently found evidence for a serious source of error that may arise in such measurements when the overlayer film is ordered.

Figure 1 shows a striking example of the observed effect. The iower curve shows XPS data for the nickel $2 \mathrm{p}_{3 / 2}$ photoelectron peak measured after 0.3 monolayers of nickel had been deposited on a Cu(100) substrate. The top curve shows similar data, recorded under the same measurement conditions, after the nickel film had been covered by 1.5 monolayers of copper. Instead of the expected decrease in the Ni signal due to electron attenuation in the $C u$ overlayer, there is a sharp increase if, for this case, the photoelectrons are collected at a polar angie of $45^{\circ}$ in the $\langle 100\rangle$ surface azimuth. For these conditions, the overlayer film acts to increase rather than attenuate the $\mathrm{Ni}$ signal.

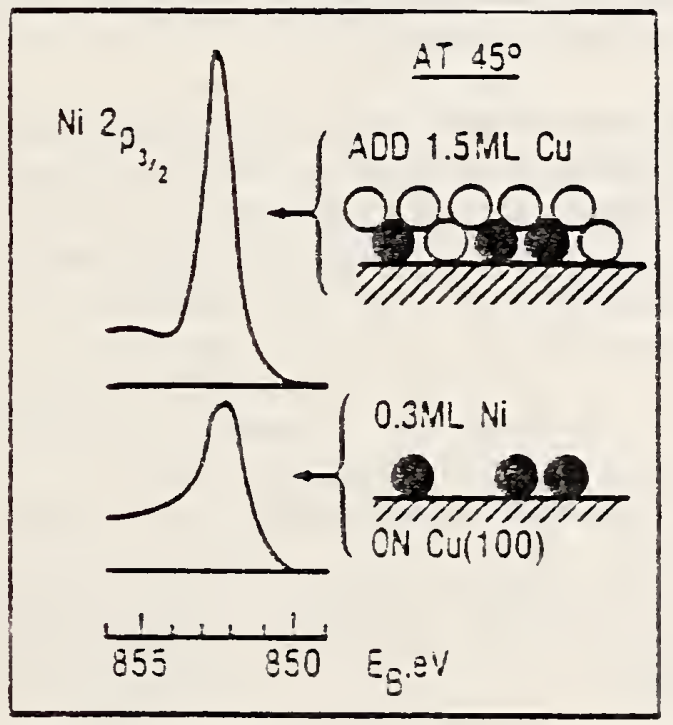

Fig. 1. X-ray photcelectron spectrum from 0.3 monolayers of nickel on 3 $\mathrm{Cu}(100)$ substrate (jottom) and following the addition of 1.5 monolayers of copper (top). 
The basis of this new phenomenon is the forward-scattering enhancement of photoelectron intensities along internuclear axes that is described in section 4.B.5. The overlying $\mathrm{Cu}$ atoms redirect the $\mathrm{Ni}$ core-level intensity from being nearly isotropic to being peaked at the $45^{\circ}$ polar angle. At other polar angles, the NI XPS signal decreases as expected.

These measurements indicate that conventional attenuation-length measurements by the overlayer-film method may have substantial errors. These errors will depend on the particular measurement geometries and the degree of crystalline order in the overlayer film. Work is in progress to determine the magnitude of the forward-scattering effect in other systems and to examine its impact in more detail on attenuation-length measurements.

8. Background Estimation in X-Ray Photoelectron Spectroscopy and Auger-Electron Spectroscopy

(W. F. Egelhofi)

A difficult problem that arises when $x$-ray photoelectron spectroscopy (XPS) and Auger-electron spectroscopy (AES) are used for quantitative surface analysis is the estimation and subtraction of the "background" intensity under the peaks of interest. No rigorous experimental or theoretical method has as yet been devised for making separations of the XPS and AES signals from the often iarge and sloping background of secondary and scattered electrons. Common practice now consists of making arbitrary (but reasonable) estimates of this background guided by inelastic electron scattering data.

A new approach to the background-estimation problem has been discovered based on the forward-scattering phenomena described in section 4.B.5. Figure 2 shows XPS data for $\mathrm{Ni}(100)$ that has been decomposed into two parts, XPS intensity which exhibits enhancement at a polar angle of $45^{\circ}$ and XPS background intensity (shaded) which does not. The basis of the decomposition is the observation that the primary photoelectron intensity is enhanced along internuclear axes whereas background electrons are emitted isotropically. Although this method of background estimation can be accomplished only with single-crystal materials, the background shape can be compared with results from electron transport calculations.

9. Theory of the Quantum Hall Effect: Application to Measurement of the Fine-Structure Constant and to Realization of a New Resistance Standard

(S. M. Girvin)

Theoretical investigations of the quantum Hall effect have been undertaken in support of the NBS Electricity Divisicn's quantized Hall resistance project. The quantization of the Hall resistance is being used to measure the fine structure constant to very high accuracy and to realize a universal standard of electrical resistance based solely on 


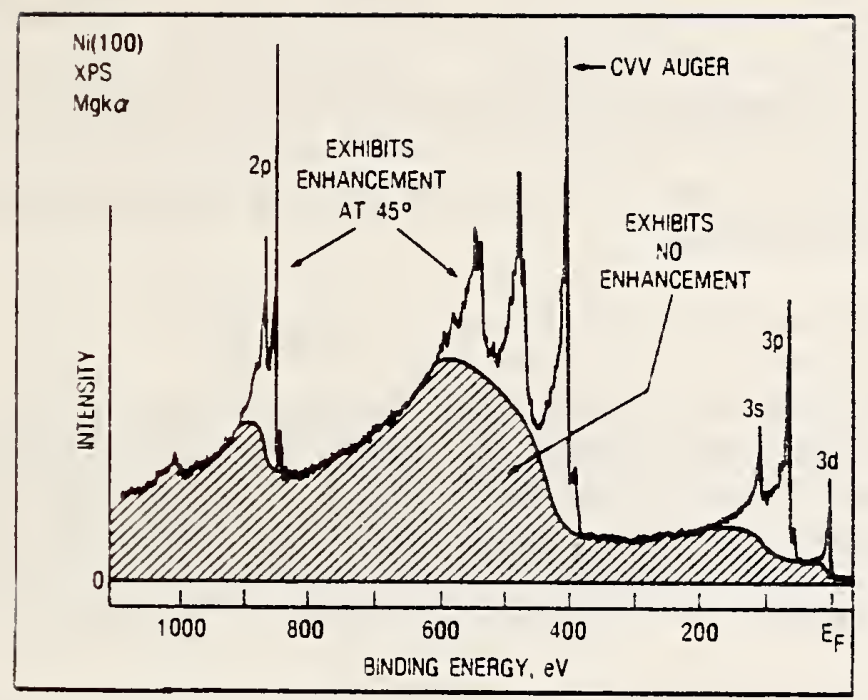

Fiz. 2. X-ray photoelectron spectrum of $\mathrm{Ni}(100)$. The shaded (background) region indicates the intensity that does not snow enhancement at a polar angie of $45^{\circ}$.

fundamental constants of nature. We have addressed both fundamental physics questions (see section 4.2 ( 8 ) as well as practical problems concerning possible sources of experimental error in the extremely high accuracy measurements being made. Recent topics of investigation in the latter category include thermoelectric effects and the role of pinite temperatures and disorder. Another important area has been the successful interpretation of the sudden, extremely non-linear onset of dissipative breakdown at high current densities. This phenomenon occurs when the carrier drift speed exceeds the speed of sound resulting in a phonon lasing epfect.

\section{Needs for RePerence Data}

The growth of surface science and its applications has led to needs for expanded reference data. These needs can be summarized in the following three categories.

\section{a. Surface Analysis}

Four techniques are now in common use for surface analysis: Auger-electron spectroscopy (AES), $x-r$ ay photoelectron spectroscopy (XPS) or electron spectroscopy for chemical and analysis (ESCA), secondary-ion mass spectroscopy (SIMS), and ion-scattering spectroscopy (ISS). With each technique, there is need for the following reference data: 
- evaluation of spectra from pure elements for common conditions of measur ement

- evaluation of chemical or matrix effects on spectra

- benchmark data for the calibration of the energy and intensity scales of instruments

- reference data on line positions in compounds

- reference data for key parameters in quantitative analysis (e.g., ionization cross sections (AES, XPS), ion scattering cross sections (ISS), electron inelastic mean free paths and attenuation lengths (AES, XPS), electron backscattering (AES), ion neutralization cross sections (SIMS, ISS), and multiple ionization (SIMS)

- algorithms for data analysis (e.g., determination of background due to multiple scattering (AES, XPS, ISS) and fragments (SIMS, ISS), deconvolution to obtain source Iineshape (AES, XPS, ISS), inelastic scattering (AES, XPS), and double scattering (ISS))

- data for ion sputtering yields in elements, alloys, and compounds as a function of ion type, ion energy, and bombardment geometry

- data for radiation damage rates during analysis, including cross sections for descrption from surfaces and decomposition of surface layers.

b. Surface Science

Many techniques are in common use for the determination of surface properties and processes. Data are needed in the following areas:

- reference data on vibrational energies and intensities for molecular species on surfaces (as measured by electron energy-loss spectroscopy, infrared spectroscopy, Raman scattering, neutron inelastic scattering)

- reference data for deternination of surface structures by low-energy electron diffraction and other techniques (e.8., library of basic data, algorithms to compute surface structure)

- reference data for absolute determination of surface coverages (atoms $/ \mathrm{cm}^{2}$ ) on surfaces of different structure and atomic composition

- reference data for surface processes (kinetic data for catalytic and surface reactions, desorption, diffusion, segregation) 
- Ion-surface interactions are of importance in sputtering and ion scattering spectroscopy. Reference data are needed to characterize the electron and photon spectroscopy of scattered and sputtered ions, as well as of excited neutral species leaving the surface.

c. Interface Science

There is rapid development now of techniques that can be used for the in situ characterization of solid-solid, solid-gas, and solid-liquid interfaces. A variety of techniques (e.g., Rutherford backscattering spectroscopy, infrared spectroscopy, Raman spectroscopy, extended $x$-ray absorption fine structure, neutron inelastic scattering, particle-induced $x$-ray emission) are being utilized and reference data and algorithms will be required for their effective application.

The Division plans to establish priorities for these data needs in collaboration with the interested groups including the NBS office of Standard Reference Data, the recently formed Standard Reference Data Subcommittee of ASTM Committee E-42 on Surface Analysis, and the Surface Chemical Analysis Project of the Versailles Agreement on Materials and Standards (section 2.D.2). A plan for generation of high-priority data will then be prepared.

B. Reference Materials

1. Development and Production of Standard Reference Materials (J. Fine)

The objective of this project is to develop standard reference materials (SRMs) that will allow convenient determinations of ion-beam sputter-removal rates in commonly used surface-analysis instrumentation. Thin film reference materials of known thickness will be used to calibrate ion-gun current densities for particular operating conditions. This information together with sputtering-yield data for a range of materials will establish depth scales in the measurement of depth profiles.

Prototype thin-film structures were initially fabricated in order to evaluate their usefulness as depth profiling SRM's and to develop appropriate characterization techniques. These structures were nade by sputter deposition as part of a joint US/Yugoslavia program between NBS and Dr. B. Navinsek at the J. Stefan Institute in Ljubljana. Analyses of these prototype materials at NBS and by members of ASTM Committee $\Xi-42$ on Surface Analysis were done using Auger-electron spectroscopy, $x$-ray fluorescence, secondary-ion mass spectroscopy, Rutherford backscattering spectroscopy, and proton-induced $x-r a y$ emission. The results were satisiactory and a stock of similar thin-film SRM's were fabricated in 1982 using the sputter deposition facility at the $J$. Stefan Institute in ¿jubljana. 
The analyses obtained on our nickel-chromium multilayered thin-film structures indicate that this material should be of considerable benefit as a depth-profiling SRM. Work on its characterization and calibration is now completed. We have determined the uniformity and periodicity of the thin-film layers, absolute film thicknesses, sputtered interface depth resolution, and structural stability. Measurement methods used to characterize this $\mathrm{Ni} / \mathrm{Cr}$ SRM include $\mathrm{N}(\mathrm{E})$ Auger sputter profiling, Rutherford backscattering spectroscopy (RBS), and neutron activation analysis (NAA).

Previous results of absolute film thicknesses and uniformity obtained by RBS indicate that samples taken from the same production batch, as well as from 3 separate batches are all uniform to \pm 3 percent for a given layer material. These values now have been further verified with NAA techniques by $\mathrm{Dr}$. R. Fleming of the NBS Inorganic Analytical Research Division. This method analyzes the total $\mathrm{Ni}$ and $\mathrm{Cu}$ content of the multilayered films by using a gravimetrically calibrated reference; the resulting accuracy is better than 1 percent. NAA results obtained on the $\mathrm{Ni}$ layers indicate that the average $\mathrm{Ni}$ layer thickness (areal density) determined from three batches is $58.3 \mu \mathrm{g} / \mathrm{cm}^{2}$ ( \pm 2 percent). The uncertainty in the film thickness variation in samples of a given batch is \pm 1 percent. The average $\mathrm{Ni}$ layer thickness as determined by NAA is 4 percent greater than the RBS value. This difference is probably due to the uncertainty in the stepping power data used in the RBS analysis; this uncertainty is generally recognized to be between 5 and 10 percent. Statistical analysis of our characterization results and calibration data is being completed. This Ni/Cr structure will be issued as NBS Standard Reference Material 2135 once the certificate has been written.

Future work includes characterization and calibration of a "marker" layer-type sputter depth profile SRM which we have recentiy fabricated in production quantities. This material also was fabricated in Dr. Navinsek's laboratory and consists of eight $\mathrm{Cr}$ layers ( - $40 \mathrm{~nm}$ each) separated by about 2 monolayers of $\mathrm{Cr}_{2} \mathrm{O}_{3}$.

\section{Quantitative Sputter Depth Profiling of Interfaces} (J. Fine)

Determination of composition-versus-depth at virgin solid/solid interfaces is at present difficult to achieve with sputter-depth-profiling techniques. This is due, to a large extent, to a lack of understanding of the dependence of the sputtered-interface depti resolution on ion bombardment conditions (i.e., ion beam energy, species, angle of incidence, current density, and on sputtered depth). A careful evaluation of such factors which affect interface resolution is not available for any given interface system. A consistent set of such measurements obtained for various ion beam parameters, all done on the same material, would significantly enhance our ability to assess the abruptness of interfaces before sputter-profile analysis took place and would be of value in determining optimum sputter-profile conditions. 
Our development of $\mathrm{Ni} / \mathrm{Cr}$ multilayered SRMs with known layer thickness and periodicity makes it both possible and practical to systematically examine the dependence of interface resolution on ion beam parameters. Use of the computer-controlled, sequential-mode $N(E)$ Auger data system whlch has been previously developed allows us to obtain reliable and accurate measurements of the interface depth resolution for the $\mathrm{Ni} / \mathrm{Cr}$ aaterial.

We have made the following measurements during the past year:

a. Sputtered interface depth data has been obtained for 1 and $2 \mathrm{keV}$ argon ion bombardment at various ion current dens:ties at $50^{\circ}$ angle of incidence as a function of sputtered depth. We have demonstrated that measured interface deptins (using the 10 to 90 percent intensity-change criterion) as narrow as $5.0 \mathrm{~nm}$ can be obtained with these SRMs.

b. In collaboration with Dr. B. Navinsek and his group at the $J$. Stefan Institute in Ljubljana we have made use of quartz crystal microbalance techniques to monitor the rate of mass-loss during sputter profiling. This method allows us to observe interface depths as evidenced by the change in sputtering rate due to the differences in the sputtering yields of $\mathrm{Ni}$ and $\mathrm{Cr}$ at an interface. Our results indicate that interface depths as narrow as $3.5 \mathrm{~nm}$ can be observed on our $\mathrm{Ni} / \mathrm{Cr}$ SRMs during sputtering with $8 \mathrm{keV}$ argon ions.

c. We also are collaborating with Dr. P. Lindfors of the Perkin-Elmer Corporation - Physical Electronics Division and $\mathrm{Dr}$. D. Mitchell of the National Research Council of Canada, Ottawa to document interface deptin resolution dependence on ion beam angle of incidence and on current density. Due to the outstanding structural composition of our SRMs, we have been able to collect a large set of data, and we will be able to define interface "shapes" as "well as interface depths. This data should be adequate to warrant calculations of the collision cascade broadening effect at sputtered interfaces.

3. Needs for Reîerence Materials

The foliowing needs for additiona: reference materials have been identified.

\section{a. Depth-Profiling SRMs}

Surface analysis is inequently combined with ion splutering to measure composition versus depth and to investizate solid-solid interfaces. It is believed that sputter-depti-profiling applications wili continue to grow and, with the development of new techniques for deposition of materials (e.j., molecular-beam epitaxy), the development of better depth-profiling techniques, and demands for better accuracy, use of 
new materials, and desire for sharper interfaces, there will be an increased demand for suitable SRMs to ensure the reliability of the relevant measurement system.

NBS has recently developed a multi-layer periodically-modulated SRM consisting of alternate layers of $\mathrm{Ni}$ and $\mathrm{Cr}$ (section 2.2 (a)). Similar structures consisting of $\mathrm{Ni} / \mathrm{Ag}$ and $\mathrm{Cr} / \mathrm{Cr}_{2} \mathrm{O}_{3}$ have been fabricated but remain to be characterized. We have also Pabricated films of $\mathrm{Ta}_{2} \mathrm{O}_{5}$ on $\mathrm{Ta}$ and these also have to be characterized.

There are needs for similar SRMs involving other types of materials in high-technology industries, for example, $\mathrm{SiO}_{2}$ on $\mathrm{Si}$, epitaxial modulated-thickness films of GaAs/GaAlAS, thin layers of metals on semiconductors (Schottky barriers), and polymer films on metal substrates.

\section{b. Calibration of Surface Analysis Instruments}

There is a growing need for the characterization and calibration of surface-analysis instrumentation, particularly since the newer computer-controlled instruments require greater system stability and reproducibility than before. Until recentiy, most surface analyses have been qualitative but there is increasing demand for quantitative analyses of defined accuracy. These developments require SRMs for instrument calibration (e.g., of the instrument transmission functions for Augerelectron spectrometers (AES) and $x$-ray photoelectron spectrometers (XPS) and for the determination of instrument performance (e.g., the detection of trace constituents).

Pure elemental and multicomponent materials can be utilized for calibration of the energy and intensity scales (and instrument transmission functions) of AES and XPS instruments. A suite of pure elements (e.g., $\mathrm{Cu}, \mathrm{Ag}$, and $\mathrm{Au}$ ) could be used for both purposes. Liquid alloys (e.g., Ga-Sn or Ga-In) can be cleaned readily to generate two-component surfaces of known compcsition for checks of instruments and algorithms for analyses. Multicomponent materials with known quantities of trace elements are needed for determining the ability of an instrument to detect trace species. Suitable materials could include ion-implanted impurities in silicon (with a specified depth distribution and maximum concentration) and multi-component liquid alloys (with homogeneous compositions or with segregation of trace species at the surface).

\section{c. Surface Science}

There is a need for SRMs that would lead, with the user following a prescription, to surfaces of known composition (e.g., to known coverage of a chemisorbed layer of molecules on a metal substrate or to a monatomic layer of a metal on a substrate). A single crystal SRM (e.g., $\mathrm{Ni}$ ) could be cleaned and exposed to different gases (e.8., $\mathrm{O}_{2}, \mathrm{~N}_{2}, \mathrm{H}_{2}, \mathrm{CO}$, $\mathrm{H}_{2} \mathrm{~S}$ ) to establish known surface coverages of such gases. Such an SRM would enable calibration of AES and XPS equipment now used for measuremerts of surface coverages. Another use of the SRM could be through the deposition of several monolayers of a metal (e.g., Ag), again after 
cleaning, with appropriate thermal treatment to remove all but a monolayer of the deposit. This process could also be used for the calibration of AES and XPS equipment used for investigations of novel properties of ultrathin metal pilms.

The Division intends to establish priorities for new SRMs in collaboration with the NBS Office of Standard Reference Materials and the Standard Reference Materials Subcommittee of ASTM Committee E-42 on Surface Analysis. Plans for producing additional SRMs will also depend on sales of the recently developed Ni/Cr SRM 2135 (section 2.2 (a)) that will be announced shortly.

C. Review Articles and Books

1. Review Articles

The Division staff authors many review articles and book chapters that provide information on measurement methods, relevant reference data, reference materials, and theories of the measurement process for many surface spectroscopies.

Division staff members have authored 24 review article recently. The titles and abstracts of review articles published since 1983 are listed below:

a. "Adsorption on Metal Surfaces: Some Key Issues," J.W. Gadzuk, in Atomistics of Fracture, eds: R. M. Latanision and J. R. Pickens (Plenum Press, NY), 391-418 (1983).

The physical picture and basic philosophy of the principal techniques for performing theoretical calculations of the electronic structure of adsorbates on metal surfaces are presented. A discussion of spectroscopic probes and non-adiabatic effects is ziven. These ideas are illustrated via specific case studies.

b. "Fundamental Excitations in Solids Pertinent to Desorption Induced by Electronic Transitions," J. W. Gadzuk, in Desorption Induced by Electronic Transitions DIET-I, eds: N. H. Tolk, M. M. Traum, J. C. Tully and T. E. Madey (Springer-Verlag, NY), 4-25 (1983).

Various aspects of the importance of treating the initial electronic excitation and subsequent atomic, ionic, or molecular nuclear moticn as a coherent single process is emphasized. Theoretical modeling is carried out in terms of Franck-Condon factors for ooth translational degrees of freedom as well as intrinsic substrate excitations. Several desorption scenarios are discussed and model desorbate enersy disiributions which follow from the theory outlined here are presented. 
c. "The Determination of Molecular Structure at Surfaces using Angle Resolved Electron and Photon Stimulated Desorption,"

T. E. Madey, F. P. Netzer, J. E. Houston, D. M. Hanson, and R. L. Stockbauer, in Desorption Induced by Electronic Transitions DIET-I, eds: N. H. TOIK, M. M. Traum, J. C. Tully, and T. E. Madey (Springer-Verlag, NY), 120-138 (1983).

We review recent data and theoretical models related to the use of angle-resolved electron and photon stimulated desorption in determining the structures of molecules at surfaces. Examples include a variety of structural assignments based on ESDIAD (electron stimulated desorption ion anguiar distributions), the observation of short-range local ordering effects induced in adsorbed nolecules by surface impurities, the influence of electron-beam damage on surface structure, and a direct comparison of ESD and PSD ion yields for the same system.

d. "The Quantum Hall Effect I," M. E. Cage and S. M. Girvin, Comments on Solid State Physics, 11, 1 (1983):

The recent surprising observation of a quantization of the Hall resistance in units of $\mathrm{h} / \mathrm{e}^{2}$ in quasi-two-dimensional conductors presents the possibility of obtaining an improved value of the fine structure constant and development of a quantum standard of resistance using a solid state device.

e. "The Quantum Hall Effect II," S. M. Girvin and M. E. Cage, Comments on Solid State Physics 11, 47 (1983).

The recent surprising observation of a quantization of the Hall resistance in units of $\mathrm{h} / \mathrm{e}^{2}$ in quasi-two-dimensional conductors has necessitated a major rethinking of our theoretical picture of transport in these systems. The central problem is understanding why ideal behaviour persists even in the presence of strong disorder.

f. "Thin Film Reference Materials for Sputter Depth Profile Calibration," J. Fine and B. Navinsek, in Proc. of Invited Lectures and Progress Reports, Symposium on the Physics of Ionized Gases, ed: G. Pichier, Institute of Physics of the University (Zagreb, Yugoslavia), 223 (1983).

Standard reference materials for caliorating depth-oferosion scales in ion beam sputtering are presently being developed jointly by the National Bureau of Standards, the Josef Stefan Institute, and ASTM Committee E-42 on Surface Analysis. Eour separate types of thin film structures have been selected and produced in order to satisfy various needs in depth profile analysis using surface spectroscopies. Advantages of these specific structures as well as details of their fabrication will be discussed. Prelininary results on the characterization and compositional analysis of these prototype materials have beer obtained regarding uniformity and periodicity of multilayered films, interface widths, absolute film thickness, film stoichiometry, and film 
stability and diffusion. The measurement methods used to characterize these thin-film structures include compositional sputter depth profiling using Auger spectroscopy as well as $x$-ray fluorescence, Rutherford backscattering spectroscopy, chemical analysis, weight-loss measurements due to sputter erosion using quartz oscillator techniques, and proton induced x-ray emission. The results of these analyses indicate that such materials may be well suited for use in sputter depth profile calibration.

8. "Electron and Photon Stimulated Desorption: Benefits and Pitfalls," T. E. Madey, D. L. Doering, E. Bertel, and R. Stockbauer, Ul tramicroscopy 11, 187 (1983).

Electron beam irradiation of solids often results in damage-producing events along with information-producing events. In the present paper we explore mechanisms of beam damage in solids, as well as examples of the benefits of electron (and photon) stimulated processes to study molecules at surfaces. Information about the geometrical structure of adsorbed species can be obtained from measurements of the angular distribution of ions released by electron or photon stimulated desorption. The directions of ion emission are directly related to the orientations of the surface bonds which are ruptured by the excitation. The method of Electron Stimulated Desorption Ion Angular Distributions (ESDIAD) has proven particularly useful in characterizing local molecular structure at surfaces in the absence of long range order; recent measurements of bonding configurations stabilized by impurities or by lateral interactions are discussed. Photon stimulated desorption (PSD) studies using synchrotron radiation are providing new insights into fundamental electronic excitation processes at surfaces. Mechanisms for the excitation and desorption of ions are examined (valence, shallow core level and deep-core-level excitations), and examples include ion desorption from adsorbed monolayers, as well as ion formation and desorption processes in condensed molecular films.

h. "An Overview of Research at NBS using Synchrotron Radiation at SURF-II," D. L. Ederer, R. P. Madden, A. C. Parr, E. B. Saloman, G. Rakowsky, J. Copper, R. Stockbauer, T. E. Madey, and J. L. Dehmer, Proc. of the Conference on the Applications of Accelerators in Research and Industry, IEEE Trars. On Nuclear Science NS30, 1020 (1983).

The National Bureau of Standards (NBS) Synchrotron Ultraviolet Radiation Facility (SURF-II) is used in conjunction with several high throughput monochromators to study the interaction of vacuum ultraviolet photons with solids and gases. Recent work has been concerned with the photon stimulated desorption of atomic and molecular ions from surfaces, with the effect of electric fields on molecular photoabsorption and with the study of molecular photoionization by angle-resolved photoelectron spectroscopy. These research programs yield new information about molecular bonding at surfaces, molecular dynamics near ionization thresholds, and the coupling of the electronic and nuclear motion near resonances in molecules. In addition to these programs in basic research, SURF-II is used for the calibration of transfer standard detectors over a 
photon energy range 20-250 eV. Calibration of monochromator systems is achieved over the photon energy range $5-250 \mathrm{eV}$ by using the now calculable spectral intensity radiated by the electrons, which are confined in a neariy circular orbit.

\section{1. "The Use of Angle Resolved Electron and Photon Stimulated}

Desorption for the Determination of Molecular Structure at Surfaces," T. E. Madey and R. Stockbauer, Rev. Brasil. Apl. Vac. (J. of Brazilian Vacuum Society) $\underline{3}, 47$ (1983).

This paper is a brief review of recent data related to the use of angle-resolved electron stimulated desorption and photon stimulated desorption in determining the structures of molecules at surfaces. Examples include a variety of structural assignments based on ESDIAD (electron stimulated desorption ion angular distributions), the observation of short-range local ordering effects induced in adsorbed molecules by surface impurities, and the application of photon stimulated desorption to both ionic and covalent adsorbate systems.

j. "Desorption of Ions from Surfaces: Mechanisms of Photon Stimulated Desorption," R. Stockbauer and T. E. Madey, Annals of the Israel Phys: Soc. 6,483 (1983).

A review is ziven of the mechanisms of Photon Stimulated Desorption (PSD) from ionic, covalent and van der Waals bonded surface molecules. An interatomic Auger decay process describes desorption from ionically bonded, maximal valency compounds. The mechanism for ion desorption from covalently bonded systems is thought to involve relatively long-lived two-hole states. Ion desorption from thick molecular films presents a challenge to theory since heavy fragments $\left(\mathrm{C}_{\mathrm{y}} \mathrm{H}_{\mathrm{y}}\right)$ desorb from some (cyclohexane) and not erom others (water, methanoi).

k. "Instrumentation for Photon Stimulated Desorption," R. Stockbauer, Nucl. Instr. Meth. 222, 284 (1984).

Photon stimulated desorption (PSD) refers to the ejection of ions, atoms or molecular fragments from a surface initiated by the absorption of single photons; to date, most studies have concentrated on detection of ions. The properties of PSD which make it attractive as a surface characterization tool are its extreme surface sensitivity, the ions being ejected only from the topmost layer and the rapidity with which the ions are ejected. Since the desorption is fast $\left(-10^{-14} \mathrm{~s}\right)$, with respect to molecular vibrations $\left(-10^{-12} \mathrm{~s}\right)$, the ion trajectory reflects the initial bonding geometry of the particle to the surface.

To study the ion desorption process, one would like to know the mass, kinetic energy distribution, angular distribution and the yield (desorption rate vs. photon energy) of the desorbing ions. Time-of-flight (TCF) analyzers are used where the time between the light pulses from the synchrotron iight source is on the same order as the flight time of the ion from the surface to the detector. One can measure easily ion mass and yield but not kinetic energy or angular distributions with this class of 
analyzer. Electrostatic deflection analyzers are used to measure ion ylelds and kinetic energy distributions and, in some instruments, ion angular distributions. In general, they are useful where the time structure of the synchrotron light is too short to allow TOF analysis. Optical methods have recently been applied to the detection of excited states of neutral Li seen in PSD of LiE.

1. "Inelastic Scattering of Electrons in Solids," C. J. Powell, in Electron Beam Interactions with Solids for Microscopy, Microanalysis, and Microlithography, D. F. Kyser, H. Niedrig, D. E. Newbury and R. Shimizu, eds. (SEM Inc., Chicago), 19-31 (1984).

The principal mechanisms and available data for the inelastic scattering of electrons in solids are reviewed. The processes relevant for electron-probe microanalysis, electron energy-loss spectroscopy, Auger-electron spectroscopy, and $x$-ray photoelectron spectroscopy are described and examples of relevant electron energy-loss data are shown. The discussion is based on the dielectric description of inelastic scattering and treats processes important in the excitation of both core electrons and valence electrons. Information is given on the cross sections for ionization of core levels, inelastic mean free paths of huger electrons and photoelectrons in solids, and radiation damage.

m. "Non-adiabatic Effects in Elementary Surface Reactions: State-to-State Molecular Beam Experiments as a Probe," J. 'N. Gadzuk, in Many-Body Phenomena at Surfaces, D. Langreth and H. Sunl, eds. (Academic Press, NY), 517(1984).

A theory of elementary chemical reactions at solid surfaces is described in terms of surface induced diabatic transitions between "reactant" and "product" potential curves. It is then shown how the internal vibrational state distribution of a diatonic molecule scattered irom a surface could provide unique dynamical information required as input to the diabatic transition theory.

n. Early Applications of Vacuum, from Aristotle to Langmuir," T. E. Madey, J. Vac. Sci. Tech. A2, 110 (1984).

Highlights of the development of vacuum science and technology from ancient times to the early twentieth century are reviewed. The view of the Greek philosophers that vacuum was an impossibility hampered understanding of the basic principles of vacuum until the mid 17 th century. Verifiable vacua were first produced in Italy by Berti and Toricelli; von Guericke's dramatic experiments vividiy demonstrated atmospheric pressure. Piston-like "air pumps" were widely used in England and the European continent through the 18 th and ear'y 19 th centuries to produce and characterize the properties of vacuum (lack of sound iransmission, inability to support life, gas discharges, etc.). The industrial Revolution was made possible through the zenius of Newcomen, who designed huge atmospheric engines (based on condensation of steam to form a vacuum beneath a piston, which was then driven by the pressure of the atmos- 
phere). A system of "atmospheric railways" propelled by vacuum pistons was built in England in the mid 19 th century. Serious scientific developments of the 19th century which necessitated vacuum included Crooke's and Faraday's gaseous discharge measurements, the first sputtering experiments by Grove, the isolation of the rare gases by Ramsey, the standards work of Miller, the discovery of the electron by Thomson and of X-rays by Rontgen. The development of the incandescent light by Edison provided a background for the remarkable achievements of Langmuir in vacuum and surface science at the dawn of the 20th century. An appendix is included which lists museums containing vacuum-related exhibits.

\section{Review Articles in publication}

We list below the titles and abstracts of review articles in the course of publication.

a. "The Chemisorption of Carbon Monoxide by the Transition Metals," J. T. Yates, Jr., T. E. Madey, and J. C. Campuszno, The Chemical Physics of Solid Surfaces and Heterogeneous Catalysis, eds: D. A. King and D. P. Woodruff.

A comprehensive review of the adsorption of carbon monoxide by transition and noble metals is presented. The systematics of $C O$ adsorption and dissociation are discussed, along with detailed examinations of the surface structures, electronic and vibrational spectra, and kinetic behavior of adsorbed $\mathrm{CO}$.

b. "The Thermal Desorption of Adsorbed Species," J. T. Yates, Methods of Experimental Physics, ed: R. L. Park.

The experimental and theoretical basis of thermal desorption spectroscopy as a tool for surface characterization is described. Methods of analysis of thermal desorption spectra to obtain kinetic information (desorption energies, desorption order, coverages of adsorbed species) are included, together with numerous examples of desorption of small molecules from single-crystal metal surfaces.

c. "Experimental Methods in Electron and Photon Stimulated Desorption," T. E. Madey and R. Stockbauer, Methods of Experimental Physics, ed: R. I. Park.

We review experimental techniques and applications of electron stimulated desorption (ESD) and photon stimulated desorption (PSD) of atomic, molecular, and ionic species from surfaces. The physical principles of these methods, as well as details of experimental measurements and data analysis are ciscussed. We close with a discussion of beam damage in surface analysis. 
d. "Laser Studies of Surface Chemical Reactions," R. R. Cavanagh and D. S. King, Chemistry and Physics of Solid Surfaces V, Springer-Verlag.

A review of laser studies of surface chemical dynamics with emphasis on thermal desorption processes is presented. The correlation of gas-phase and liquid-molecular dynamics with analogous surface processes is demonstrated with primary emphasis on experimental techniques for monitoring relevant quantum state populations. Recent results for the desorption of NO from Ru(001) and NO from oxidized Ru crystals are compared in terms of rotational populations, and velocity and angular flux distributions.

e. "Final-State Resolved Studies of Molecule-Surface Interactions," D. S. King and R. R. Cavanagh in New Laser and Optical Investigations of Chemistry and structures at Interfaces, Verlag Chemie.

Quantum state-specific diagnostics as applied to surface dynamics are discussed. The NO molecule is used as an illustration of the experimental determination of gas-surface interactions. Topics reviewed included beam-surface scattering, thermal desorption, vibrational accommodation, Doppler profiles, and Fourier-transform infrared emission spectroscopy.

f. "Characterization of Surfaces Using Electron and ?hoton Stimulated Desorption," T. E. Madey, D. E. Ramaker, and R. Stockbauer, Ann. Rev. Phys. Chem.

We review various mechanisms of electron- and photonstimulated desorption of ions and neutrals from surfaces. Examples include desorption from ionic surfaces, from covalent adsorbates on metal surfaces, and from layers of physically adsorbed atoms. The use of the electron stimulated desorption ion angular distribution (ESDIAD) method for determining local structures of surface molecules is described.

g. "Inelastic Mean Free Paths and Attenuation Lengths of Low-Energy Electrons in Solids," C. J. Powell, Scanning Electron Microscopy/1984.

A review is given of calculations of inelastic mean free paths and measurements of attenuation lengths of low-energy electrons in solids. The emphasis of the review is on the systematics of the dependences of these quantities on material and electron energy. Calculations of inelastic mean free paths from experimental dielectric data indicate that different dependences on electron energy occur in different materials and that deviations from simpie theoretical or empirical expressions are to be expected. 
h. "Innershell Ionization Cross Sections," C. J. Powell in Electron Impact Ionization, T. D. Märk and G. H. Dunn, eds., Springer-Verlag.

A review is presented of cross-sections for the lonization of inner-shell electrons by electron impact. Calculations, measurements, and empirical formulas are described and compared. Recommendations are given for the selection of cross-section data. Finally, applications of inner-shell ionization cross-section data to materials and surface analysis problems are described.

i. "Core Level Binding-Energy Shifts in Solids at Surfaces," W. F. Egelhoff, Jr., Surface Sci. Reports.

This review presents an overview of the theory and of various successful approaches to the interpretation of core-level binding-energy shifts observed in photoelectron spectroscopy. The theoretical background is presented at a level readily accessible to the general reader. Particular attention is paid to relative merits of the two basically different conceptual frameworks for interpreting core-level binding-energy shifts, the initial-state-final-state approach and the equivalent-core-Born-Haber-cycle approach.

j. "Methods for Producing Clean Liquid Surfaces," J. Fine and S. Hardy, in Treatise on Clean Surface Technology, K. L. Mittal, ed.

A review of techniquas which have been used to produce atomically clean surfaces on liquids is presented. Methods are discussed for the handling and pre-cleaning of liquid specimens. Ultrahigh vacuum techniques for obtaining clean liquid surfaces are divided into two types: those which do so by creating a new surface and those which act by removing impurities that are present. The techniques include a number of variations on drawing pure liquid from the interior of a reservoir, deposition of liquid films, skimming, evaporation, dissolution, chemical reactions, and sputtering by ion bombardment. A number of specific liquid systems are discussed.

3. Books

(C. J. Powell, T. E. Madey)

While there are many books and review articles on different aspects of surface science and its applications, there is a dearth of information on how to make reliable surface-characterization measurements. There is, in essence, a large inpormation gap between review articles in which the latest scientific advances are discussed and manufacturers' handbooks in which the operation of a commonly used surface-characterization equipment is described. Arrangements have been made with a commercial puolisher to produce a series of books, "Methods of Sunface Characterization," which will address this need. Two Division 
staff members (Powell and Madey) and three other scientists (A. $N$. Czanderna at the Solar Energy Research Institute; D. M. Hercules and J. T. Yates at the University of Pittsburgh) constitute an editorial board that w11 oversee the production. A series of four volumes is currently planned which will describe the principles, techniques, and methods considered important for surface characterization. It is intended to describe how important surface-characterization measurements are made and how to ensure that the measurements and interpretations are satisfactory (to the greatest extent possible). The approach of the series will be pedagogical or tutorial.

Volumes 1 and 2 of the book series will describe the techniques and methods for electron spectroscopy and ion spectroscopy, respectively, which are in widespread use for surface analysis. These volumes will be concerned largely with techniques for which commercial instrumentation is available. It is hoped that writing for Volume $i$ will be completed in 1984 and that for Volume 2 by early 1985.

Volume 3 will describe techniques for specimen handling and depth proplling. For specimen handling and treatment, this volume will provide a compliation of methods that have proven useful. This volume will also address the common artifacts and problems associated with the bombardment of solid surfaces by electrons and ions. Einally, a description will be given of methods for depth profiling. Authors have been selected to write chapters for this volume.

Volume 4 will bring together a description of methods for vibraticnal spectroscopy of molecules on surfaces. Most of the techniques are currently under active development; commercial instrumentation is available for some techniques but this situation could change in the next few years. The current state-op-the-art of each technique will be described as will the relative capabilities. An important component of the volume will be the summary of the relevant theory. Authors have been selected to write chapters for this volume.

D. Standards-Commi ttee Activities

Three staff members of the Division hold offices on two ASTM Committees, the E-42 Committee on Surface Analysis and the D-32 Committee on Catalysts, and on the recently approved Surface Chemical Analysis iorking Party established under the auspices of the Versailles Agreement on Materials and Standards. Participation in the activities of these groups provides an opportunity for leadership, for dissemination of NBS expertise, and to learn of significant measurement problems in relevant areaz.

1. ASTM $\Xi-42$ Committee on Surface Analysis

(C. J. Powell, J. Fine)

The ASTM Committee E-42 on Surface Analysis founded in 1975, now has scme 140 members; in addition, a large number of non-memoers, joti within the U.S. and throughout the world, participate in Committee activities. The purpose of the Committee is to advance the pield o? 
surface analysis and the quality of surface analyses through the development of appropriate standards, standard practices, reference data, reference materials, round robins, symposia, workshops, and publications. There is substantial activity in all of these areas.

Committee activities of the past year include approval of the following standards:

- E673-84: Standard Definitions of Terms Relating to Surface Analysis (includes additions of terms for AES, ESCA, and SIMS)

- E983-84: Standard Guide for Electron Beam Effects in Auger Electron Spectroscopy

- E984-84: Standard Guide for Identifying Chemical Effects and Matrix Effects in Auger Electron Spectroscopy

- E995-84: Standard Guide for Background Subtraction Techniques in Auger Electron Spectroscopy

- E996-84: Standard Practice for Reporting Data in Auger Electron Spectroscopy

- E1015-84: Standard Practice for Reporting Spectra in ESCA (Electron Spectroscopy for Chemical Analysis)

- E1016-84: Standard Practice for Determining and Specifying the Properties of Electrostatic Electron Spectrometers

The following proposed standards are at various stages of Committee or Subcomittee ballot:

- Additions to Standard Definitions of Terms Relating to Surface Analysis (Por ESCA and Energetic Ion Analysis methods)

- Standard Guide for Specimen Handling

- Standard Practice for Reporting Depth Profile Data in Secondary Ion Mass Spectrometry

Other standards are being drafted.

The Committee sponsors two symposia each year, one at the annual Pittsburgh Conference on Analytical Chemistry and Applied Spectroscopy in the spring and the other at the National Symposium of the American Vacuum Society in the fall. Papers presented at these symposia are published. A workshop is held at each meeting to discuss particular measurement problems. At the 1983 American Vacuum Society Symposium the topic was "Case Studies in Ion Sputtering: Applications and Limitations," and the topic at the 1984 Pittsburgh Conference was "Solving Industrial Problems Using Surface Analysis." 
Technical Subcommittees of the E-42 Committee have organized a number of round robins to compare the results of nominally identical measurements in different laboratories. The following round robins are currently in progress :

- Comparison at the relative intensities of Auger-electron peaks in a series of $\mathrm{Cu}-\mathrm{Au}$ alloys

- Comparisons of depth distributions measured by secondary-ion mass spectroscopy (SIMS) for ${ }^{11} \mathrm{~B}$ implanted in $\mathrm{Si}$

- Comparisons of the relative intensities of peaks in SIMS data using metallic glasses

- Comparisons of peak intensities determined by ion-scattering spectroscopy (ISS) for $\mathrm{Cu}-\mathrm{Ag}-\mathrm{Au}$ alloys

Comparisons of elemental sensitivity factors for ISS signals of $\mathrm{Cu}$ and $\mathrm{Au}$

- Comparisons of core-level binding energies determined by $x-r a y$ photoelectron spectroscopy with instruments calibrated using a field-emission electron source.

The E-42 Committee created a Standard Reference Data Subcommittee during the past year. This new Subcommittee will: (i) establish needs for reference data in the area of surface analysis; (ii) develop criteria for the evaluation of data; (iii) set priorities for needed data evaluations; and (iv) identify groups and experts to perform the evaluations. It is anticipated that the Subcommittee will give advice on these matters to the Division and the NBS Office of Standard Reference Data.

Work at NBS on the production and characterization of standard reference materials for surface analysis (see section 2.2) and on other surface-standards activities is reported to and disseminated by the $\mathrm{E}-42$ Committee.

2. Surface Chemical Analysis working Party of the Versailles Agreement on Materials and Standards

(C. J. Powel1)

The "Versailles Agreement" arose from a meeting of the Heads of Government (Economic Summit) or industrialized nations at Versailles in 1982. The participants represented Canada, Federal Republic of Germany, France, Italy, Japan, United Kinsdom, United States of America, and the Commission of the European Communities. A Sumit Working Group on Technology, Growth, and Employment was establishing consisting of government science advisers. This group has established Working Groups in areas such as photovoltaic solar energy, iocd technology, advanced robotics, biotechnology, high speed trains, remote sensing from space, and 
advanced materials and standards. A summary of developments resulting from the Versailles Agreement was published as a News and Comment article recently in Science 224, 1317 (1984).

The Versailles Project on Advance Materials and Standards (VAMAS) has considered the establishment of Technical Working parties in specific materials areas. A proposal for a Surface Chemical Analysis Working Party was prepared by Dr. M. P. Seah of the UK National Physical Laboratory and $\mathrm{Dr}$. C. J. Powell of NBS and approved by the VAMAS Steering Committee in June, 1984. The proposal described the growth and diversity of surface analysis in the development of advanced materials in modern technologies and, additionally, the use of surface analysis for improved films and coatings. The principal techniques of surface analysis in common use were identified and the technical limitations to accurate surface analyses summarized. Accurate surface analyses require: (i) knowledge of the principles of the measurement method, ( $i i)$ knowledge of the behaviour of the measuring instrument, and (iii) correct interpretation and expression of the final measured result. Standards for the measurement of surface composition with known accuracy and for the reliable determination of chemical state will thus require: (a) a base of principles, definitions of terms, and suitable equations, (b) reference procedures for the measurement of intensities and spectral features, (c) procedures and data for instrument calibrations, (d) reference data for material parameters such as elemental sensitivity factors, matrix terms, chemical state spectra, ion sputtering rates, etc., and (e) standards for specifying an analysis. Specific needs were identified for the common methods of surface analysis, Auzer-electron spectroscopy, x-ray photoelectron spectroscopy, and secondary-ion mass spectrometry together with the needs for ion sputtering which is used to obtain composition versus depth information in films and coatings. Existing standards activities in the member countries were reviewed and suggestions were made for additional standards for surface chemical analysis by scientists in the member states of VAMAS.

As yet, the VAMAS Steering Committee has not developed appropriate policies concerning the mechanisms of operation of the different Working Parties. Although the precise nature of the relationship between national groups (such as the ASTM E-42 Committee) and VAMAS is not clear, it appears that VAMAS will provide the means to develop surface analysis standards on an international basis and with support from a high level in each government. There should therefore be greater participation in the development of surface analysis standards than would happen through the ASTM E-42 Committee alone. At present, technical representatives in the VAMAS nations are being contacted to identify areas for technical activities and possible experts who might contribute. Current activities of the ASTM E-42 Committee will be coordinated with corresponding VAMAS activities. 
3. ASTM D-32 Committee on Catalysts

(R. D. Kelley)

The activities of the ASTM Committee D-32 on Catalysts are concerned with the physical and chemical properties of commercial

catalysts and with developing suitable procedures for catalytic activity measurements. The membership of the Committee numbers approximately 120. Semi-annual meetings are attended by between 60-80 percent of the membership including West Europeans and South Americans. The Subcommittee on Chemical Analysis is chaired by R. D. Kelley.

In 1984, the committee celebrated the tenth anniversary of its founding and continues to maintain an active pace. Since its inception the committee has focused its activities on tests which are used routinely in the buying and selling of catalysts. The types of tests which have been examined include surface area, pore-size distribution, density, crush strength, particle size, attrition and abrasion, chemical analysis, amongst others. These tests involve methods which were thought to be "well established", and thus particularly suitable for standardization. However, experience has shown that many of these tests have not yielded satisfactory results in round robin testing. Two examples of these problems can be seen in the standardization of the multipoint BET method for surface area and the chemical analysis for platinum in reforming catalysts. In both of these cases, standardization activity in progress for about six years and involving many problems in both procecures and materials has been substantially completed in 1984.

Highlights of the past year include:

the certification of surface area standards by the NBS Office of Standard Reference Materials (to be available shortly) and the initiation of a program to certify a platinum-containing catalyst reference materials (to be completed in $1-2$ years).

- completion of satisfactory round-robin tesing for the chemical analysis of nickel (a wet chemical method and an atomic absorption method) and of palladium (wet chemical method) in catalysts.

- initiation of standarcization of a procedure for multi-element chemical analysis of cataiysts by atanic absorption, an ambitions project which will be of great value to both producers and users of catalysts.

- Twenty test methods have been submitted for committee ballot. 


\section{SURFACE MEASUREMENTS PROGRAM}

The Division undertakes a number of projects directed to the improved physical and chemical characterization of surfaces and surface processes. These projects meet Division objectives through the development of new measurement techniques, improvement of existing techniques, development of theories of the measurement process, applications to important scientific and national problems, development of measurement procedures, tests of the measurement system, development of new concepts, and provision of needed data.

A. Catalytic Surface Chemistry: Reaction Kinetics at High Pressures over Single Crystal Catalysts

(T. E. Madey, R. D. Kelley, T. J. Udovic, M. J. Wax)

The overall objective of this research is to provide a molecular understanding of heterogeneous catalytic chemistry. Among the areas studied are the catalytic synthesis of hydrocarbons from carbon monoxide and hydrogen on metal surfaces, the mechanism of catalytic poisoning and promotion, and the molecular and electronic nature of chemisorbed species. Results of the past year are described below:

We have completed a study designed to address a controversy concerning the catalytic activity of tungsten as a methanation catalyst. We had previously found that polycrystalline $W$ wire exhibits a high activity for $\mathrm{CH}_{4}$ synthesis from $\mathrm{H}_{2}$ and $\mathrm{CO}$, whereas van Langeveld and Ponec have recently reported that evaporated $W$ films are very poor methanation catalysts. In neither of these studies were surface analysis techniques used to ascertain the surface composition of the catalyst. In an attempt to resolve this controversy, a closer look was taken at the methanation activity of $W(110)$ (the most densely-packed crystal face of $W$ ) over a range of reactant partial pressures and temperatures in conjunction with pre- and post-reaction surface analysis utilizing Auger electron spectroscopy (AES). The results of this study indicate that the $W(110)$ surface does, indeed, have the ability to hydrogenate co to form methane as well as higher hydrocarbons, but the appearance of this activity requires higher hydrogen partial pressures than were utilized by van Langeveld and Ponec. Figure $3(a)$ illustrates the methanation behavior of $W(110)$ as compared with that of $\mathrm{Ni}(100)$ determined earlier in this laboratory. The plot of turnover number for methane production versus inverse temperature indicates a lower apparent activation energy for $W(110)$ ( $56 \mathrm{~kJ} \mathrm{~mol}^{-1}$ ) compared to that for $\mathrm{Ni}(100)\left(103 \mathrm{~kJ} \mathrm{~mol}^{-1}\right)$ as well as a higher activity for $W(110)$ at lower temperatures. The plot of methanation turnover number versus partial pressure of hydrogen, figure $3(b)$, indicates the strong dependence on hydrogen partial pressure for $W(110)$ compared to $\mathrm{Ni}(100)$. For low partial pressures of hydrogen (ca. 1 Torr), the regime studied by van Langeveld and Ponec, W(110) behaves like a poor methanation catalyst. For higher hydrogen partial pressures (ca. 100 Torr), the regime previously studied in this laboratory, $W(110)$ behaves like a good methanation catalyst. These results demonstrate the importance of analyzing a wide range of reaction parameters when characterizing the catalytic activity of a surface. 

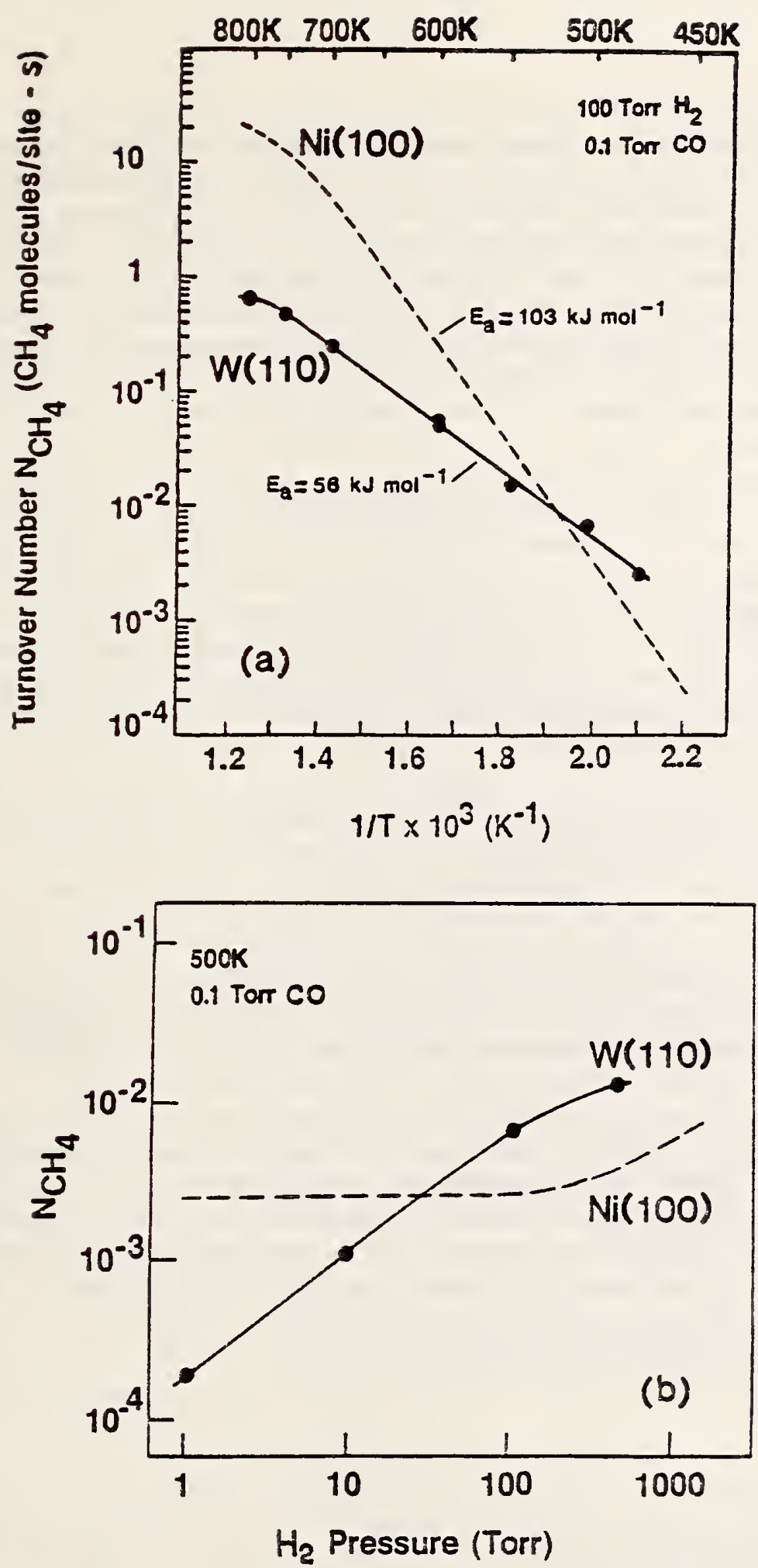

Figure 3. (a) Methanation activity as a function of temperature for $\mathrm{W}(110)$ and $\mathrm{Ni}(100)$ (partial pressure of hydrogen $=100 \mathrm{Torr}$ and partial pressure of carbon monoxide $=0.1$ Torr). (b) Dependence of methanation turnover number on the partial pressure of hydrogen for $\mathrm{W}(110)$ and $\mathrm{Ni}(100)$ (reaction temperature $500 \mathrm{~K}$, partial pressure of carbon monoxide $=0.1$ Torr). 
Research has been initiated on the reactivity of model supported catalysts. Equipment has been constructed which allows controlled deposition of aluminum and platinum on tungsten single crystals. After each experiment, a clean tungsten surface may be obtained by heating above the desorption temperature of any deposited materials. Various amounts of platinum, used commercially for hydrocarbon reforming, may be evaporated directly onto tungsten, or onto thin films of alumina prepared in situ. Use of these well-defined materials will allow examination of catalytic activity as a function of support structure and of catalyst electronic structure, which, at least in the submonolayer region, varies with platinum coverage.

We plan to examine the hydrogenolysis activity of platinum deposited on tungsten and on thin layers of alumina on tungsten. We will vary the Pt particle size by appropriate heat treatment and attempt to correlate activity with particle size. We will also complete a study of the hydrogenolysis activity of two single-crystal tungsten surfaces, (110) and (100). In addition, we will study the hydrogenation of co over refractory metal surfaces (Mo single crystals) and over alloy catalyst surfaces (e.g., Cu/Ni single crystals).

B. Electron-Stimulated Desorption: Relation to Surface Molecular Structure and Reactivity

(T. E. Madey, F. P. Netzer, K. Bange)

A continuing challenge in surface science is the determination of the structures of molecules on surfaces, particularly in the absence of long range order. The goal of this project is to provide a direct determination of the structures of adsorbed molecules using the Electron stimulated Desorption Ion Angular Distribution (ESDIAD) method, and to characterize the fundamental electronic excitations resulting in the stimulated desorption of ions. ESDIAD has the advantage of being sensitive to the local bonding geometry of adsorbed molecules, even in the absence of long range order in the adsorbed layer. The present primary thrust of this project is to use ESDIAD to characterize the influence of surface additives (e.g., catalyst promoters and poisons) on the local structure of small molecules on metal surfaces. Another objective is to determine molecular structures in controversial cases. 
During the last year, we found clear evidence for a local interaction between $\mathrm{CO}$ and $\mathrm{Na}$ adsorbed on Ru(001). For low coverages of $\mathrm{Na}\left(\theta_{\mathrm{Na}} \leq 0.15\right.$ ML) and saturation coverages of $\mathrm{CO}$ at $80 \mathrm{~K}$, a fraction of the CO molecules undergo a substantial change in bonding configuration: molecular co bound perpendicular to the $\mathrm{Ru}(001)$ surface changes to an "inclined" configuration in the presence of low coverages of $\mathrm{Na}$. The bonding interaction which causes the tilting is believed to be primarily electrostatic in nature.

We also examined the influence of two electronegative additives, 0 and $\mathrm{Br}$, on the structure and reactivity of $\mathrm{H}_{2} \mathrm{O}$ adsorbed on an $\mathrm{Ag}(110)$ surface. The interactions of $\mathrm{Ag}+\mathrm{O}$ and $\mathrm{Ag}+\mathrm{Br}$ with $\mathrm{H}_{2} \mathrm{O}$ are of interest in electrochemistry, and this study has provided a new view of the structure and kinetics.

We found that $\mathrm{H}_{2} \mathrm{O}$ adsorbed on clean $\mathrm{Ag}(110)$ forms hydrogen-bonded arrays having short-range local order; $\mathrm{H}_{2} \mathrm{O}$ desorbs in molecular form at $170 \mathrm{~K}$, with no evidence for dissociation. When $\mathrm{H}_{2} \mathrm{O}$ is coadsorbed with atomic $\mathrm{O}$ on $\mathrm{Ag}(110)$, there is evidence for new oxygen-stabilized molecular $\mathrm{H}_{2} \mathrm{O}$ having different local structure, as well as a reaction between $\mathrm{H}_{2} \mathrm{O}$ and $\mathrm{O}$ to form $\mathrm{OH}$, i.e., $\mathrm{H}_{2} \mathrm{O}+\mathrm{O}(\mathrm{ad}) \rightarrow 2 \mathrm{OH}(\mathrm{ad})$. In addition, the $\mathrm{H}_{2} \mathrm{O}$ interacts so strongly with molecular $\mathrm{O}_{2}(\mathrm{ad})$ that dissociation of $\mathrm{O}_{2}$ and formation of $\mathrm{OH}$ appear to occur even at - $100 \mathrm{~K}$. The $\mathrm{OH}(\mathrm{ad})$ is "tilted" on $\mathrm{Ag}(110)$, with the molecular axes inclined along [001] azimuths. Desorption of $\mathrm{H}_{2} \mathrm{O}$ due to recombination of $\mathrm{OH}(\mathrm{ad})$ occurs at $-300 \mathrm{~K}$.

In contrast, there is no hydrogen-abstraction reaction between $\mathrm{H}_{2} \mathrm{O}$ and $\mathrm{Br}$ on $\mathrm{Ag}(110)$. The $\mathrm{H}_{2} \mathrm{O}$ forms locally-ordered structures, and the binding energy increases, but no reaction to form $\mathrm{OH}$ (or $\mathrm{HBr}$ ) occurs.

We have used ESDIAD to address a controversy concerning the structures of atomic and molecular oxygen on $\mathrm{Ag}(110)$. Many previous authors have suggested that both $\mathrm{O}$ and $\mathrm{O}_{2}$ are adsorbed in the troughs, in high symmetry positions; $\mathrm{O}_{2}$ was believed to be bonded with the 0-0 axis parallel to the surface. Our data indicate that both atomic oxygen ( $T$ ads $>300 \mathrm{~K}$ ) and molecular oxygen ( $\mathrm{T}_{\text {ads }}=80 \mathrm{~K}$ ) are bonded in directions along [001] azimuths. The structures based on these measurements (e.8., molecular $\mathrm{O}_{2}$ bonded via one $\mathrm{O}$ atom, and "inclined" slightly in the [001] direction) are at variance with most existing models, and provide new insights into the $\mathrm{Ag}$-oxygen interaction.

There appear to be major differences in the "activity" of oxyzen in promoting hydrogen abstraction on different surfaces. On Ru(001), for example, the interaction of $\mathrm{H}_{2} \mathrm{O}$ or $\mathrm{NH}_{3}$ with adsorbed oxygen causes an increase in molecular binding energy, but no dissociation. On $N i(110)$ and $\mathrm{Ag}(110), \mathrm{O}(\mathrm{ad})$ abstracts hydrogen from $\mathrm{H}_{2} \mathrm{O}$ to form $\mathrm{OH}(\mathrm{ad})$. During the coming year, we will examine the ability of both atomic and molecular $\mathrm{O}_{2}$ to promote hydrogen abstraction reactions on different surfaces. Specifically, we will study $\mathrm{NH}_{3}+0$ on $\mathrm{Ag}(1 i 0)$, where the oxygen is relatively 
weakly bound, and $\mathrm{NH}_{3}+\mathrm{O}$ on $\mathrm{Fe}(100)$, where the oxygen is more strongly bound. Other polar molecules $\left(\mathrm{H}_{2} \mathrm{O}, \mathrm{CH}_{3} \mathrm{OH}\right)$ and other electronegative additives $(\mathrm{Br})$ will also be studied.

Iron is an excellent catalyst in $\mathrm{NH}_{3}$ synthesis, and adsorbed $\mathrm{K}$ is known to "promote" the reaction by increasing the rate. In an attempt to understand at the molecular level how $K$ influences the bonding geometry of $\mathrm{NH}_{3}$ to $\mathrm{Fe}$, we will use ESDIAD to examine the structure and reactivity of $\mathrm{NH}_{3}$ on clean $\mathrm{Fe}(100)$, and on $\mathrm{K}$-dosed $\mathrm{Fe}(100)$. The influence of $\mathrm{K}$ on the local structure of $\mathrm{CO}$ will also be studied; such interactions may be relevant to Fischer-Tropsch synthesis.

Recent angle-resolved ultraviolet photoemission spectroscopy measurements have indicated that molecular $\mathrm{O}_{2}$ is bound to a $\mathrm{Cu}(110)$ surface through one 0 atom, with the $0-0$ axis perpendicular to the surface. These results seem to be at variance with previous measurements, in which the $\mathrm{O}_{2}$ was presumed to be lying down. We will attempt to verify this structure using ESDIAD; we will also study the influence of additives $(O, B r)$ on the structure and reactivity of $\mathrm{H}_{2} \mathrm{O}$.

We will examine the structure of $\mathrm{H}_{2} \mathrm{O}$ on $\mathrm{Cu}(110)$ and the influence of additives ( $\mathrm{Na}, \mathrm{Br}, \mathrm{O})$ on the $\mathrm{H}_{2} \mathrm{O}$ structure; these measurements will be compared with ongoing theoretical calculations of the same system by $D r$. U. Landman of the Georgia Institute of Technology (and recently appointed part-time Expert to the Division staff).

C. Nucleation, Growth and Surface Atomic Structure of Ultrathin Filins

(A. J. Melmed, A. Ciszewski)

There is considerable interest in the nucleation and growth of ultrathin films of metals and semiconductors. The interest is concerned with relationships between particular physical and chemical properties and the morphology and atomic structure of the film, particular for thicknesses in the monolayer range. For example, surface atoms of samarium metal have been found to be divalent, while the bulk atoms are trivalent, and this has led to the suggestion that the surface lattice parameters should be larger than the corresponding bulk values or that surface reconstruction should occur. In order to investigate the surface atomic structure of $\mathrm{Sm}$, we determined the conditions for producing clean $\mathrm{Sm}$ surfaces by epitaxial crystal growth from the vapor. The initial work was done in a field electron microscope, and then the methods developed were transferred to a low-energy electron diffraction apparatus, where (0001) $\mathrm{Sm}$ crystal layers were grown on a (011) W substrate. Initial results indicate that the (0001) Sn surface is not reconstructed, but that is is expanded relative to the bulk by $4-15 \%$, depending on layer thickness. We plan to complete the determination of surface lattice parameter for (0001) Sm during the coming year and to clarify the homogeneity of Sin coverage during the early stages of deposition on (011) $\mathrm{W}$. 
During the past year, methods developed earlier to prepare clean flims by epltaxy from the vapor phase in the ultrahigh vacuum of a fleld-electron microscope were extended to relatively high-vapor-pressure materials. In collaboration with Drs. V. Maurice, O. Frank, and J. H. Block of the Fritz-Haber Institute in Berlin, the rare-earth metal europium was grown on rhenium and tungsten substrates. Growth modes, nucleation sites, and epitaxial relationships were determined. An unexpected type of film growth was observed to occur, namely the growth of fcc europlum on one and only one rhenium plane $[\operatorname{Re}(203)]$; this growth mode could be correlated with the surface atomic structure of $\operatorname{Re}(203)$.

D. Electron Spectroscopy of Surfaces

(T. J. Jach)

Preliminary experiments have been conducted in an attempt to observe the surface shifts of core-level binding energies by means of glancing-incidence $x$-ray photoemission. In collaboration with Drs. P. Cowan of the NBS Quantum Metrology Group and L. B. Holdeman (COMSAT), measurements have been made of photoelectron spectra of nickel and gold for $x-r a y$ angles of incidence less than and greater than the macroscopic critical angle for total external reflection in order to observe shifts in binding energies for predominantly surface or bulk atoms. Recent work has centered on the requirement to have smooth surfaces at an atomic level in order to get a significant contribution from the surface atoms. The correlation of the $x$-ray reflectance of the surface with other means of roughness measurement (stylus, scanning electron microscopy) may lead to a new method of measuring average surface roughness where the scale of the roughness is less than 20 A.

E. Ion-Surface Collisions and Atomic Excitations

(J. Fine, R. Klein)

Energetic ion-surface collisions generate extensive near-surface collision cascades in a solid. The energy transfer and atomic excitations that take place as a result of such collisions are not weil understood in part because, until rather recently, the primary method for monitoring these collision phenomena was to measure macroscopic effects such as the toral sputtering yield. The collision cascade causes the ejection of atoms, ions, electrons, and photons from the surface but it has only been in the past few years that measurements of the ejected particles or radiation have been utilized to obtain detailed information of specific atomic collisions that take place in condensed materials.

In recent experiments in which aluminum or silicon targets were bombarded by argon ions, we have shown that the Auger decay of collisionally excited Al or Si atoms can take place outside the solid depending on the distance from the surface (within the material) at which the excitation occurred, the atom's velocity, and the lifetime or the atomic excitation. As a result of our Monte-Carlo calculations to analyze these experiments, we expect that such sputtered atoms will have racher high kinetic energies - extending up to hundreds of electron volts. An experimental determination of the kinetic energy distribution and state 
identification of those specific atoms which have undergone Auger decay would give more information concerning the atomic excitation and collisional energy transfer mechanisms of ion collisions in solids.

In collaboration with Drs. L. J. Moore (NBS Inorganic Analytical Research Division) and T. B. Lucatorto (NBS Radiation Physics Division), a new series of experiments has been planned and designed to determine the kinetic energy distributions of specific sputtered atoms (ions) and their states of excitation. Resonance multiphoton laser-ionization schemes have been developed to detect sputtered atoms in specific excited states and time-of-flight techniques will be used to measure their kinetic energy distributions. Pulse timing and gating sequences have been designed and estimates made of signal intensity indicate that such measurements are feasible. An apparatus has been designed for the preliminary investigation of this problem. An ultrahigh vacuum chamber for ion-beam-angle dependent measurements is under construction and will be coupled to two ultravioiet pulsed laser systems. We plan to investigate atoms and ions sputtered from surfaces of $\mathrm{Mg}, \mathrm{Al}$, and $\mathrm{Si}$ and to study the distance beneath the surface at which collisional excitation occurs.

F. Surface Theory

(J. W. Gadzuk)

The theoretical physics projects of the Division are focused on understanding both static and dynamic behavior of atoms, molecules, solids, surfaces, radiation and their mutual interactions. This understanding comes from consideration of the microscope atomic-scale properties of individual entities as well as from larger-scale statistical properties of ensembles. Based upon our studies, phenomenological model theories are constructed which relate to the chemical physics and statistical mechanical behavior and to the intrinsic properties of relevant physico-chemical systems. In addition, a significant effort is invested in the study of the role of an actual measurement process in determining observed quantities in different surface spectroscopies. Ways in which measurement-process-specific quantities such as line shapes, satellite structures, etc. provide additional information on system dynamics are of considerable interest.

A large-scale theoretical effort has been underway aimed at the modeling of reaction dynamics at metallic surfaces and at interfaces of electro-chemical significance. Major focus in the past has been on the role of internal degrees of freedom (vibrational, rotational, and electronic) of molecules in determining energy flow and redistribution occuring in elementary nolecular processes at surfaces. Several major advances deserve mention here.

An analytic and exact model which enables calculation of the classical trajectories for a diatomic molecular beam incident upon a surface has been constructed in collaboration with Dr. S. Holloway of the University of Liverpool, England. Account is taken of the possibility for the molecule to: i) scatter in a vibrationaliy excited state; ii) form an adscrbed negative-molecular-ion resonance state; iii) dissociatively 
adsorb; and iv) undergo electronic charge transfer than thus potential surface hopping. In the spirit of past gas-phase work of Polanyi, we have explored the ramifications of potential-energy-surface (PES) topologicai features in determining the outcomes of dynamic processes at surfaces. For instance, the PES shown in Fig. 4 has been useful in studying the branching ratios for competing processes:

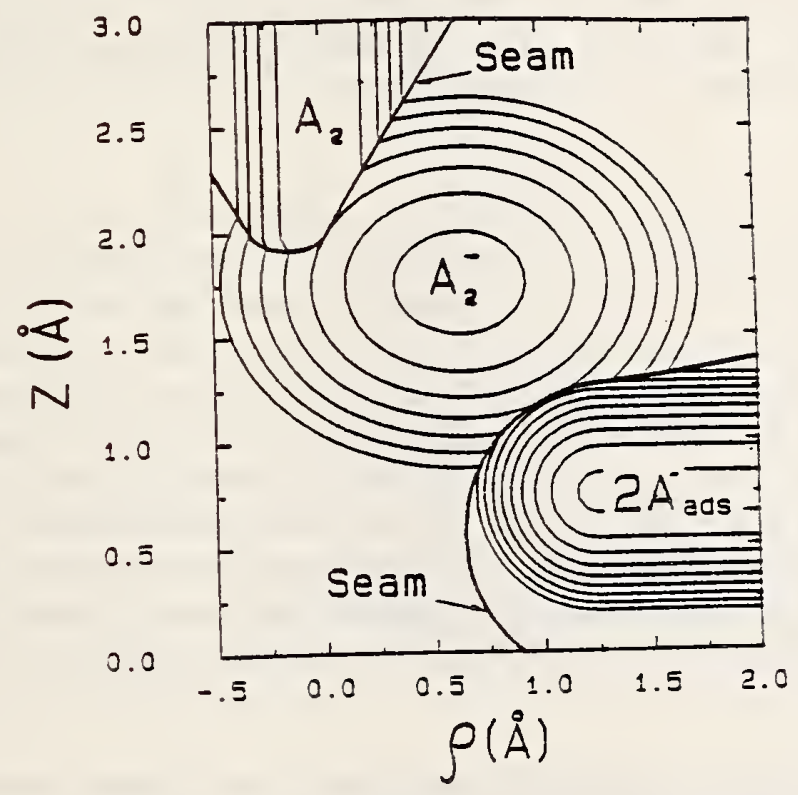

:18. 4 Diabatic potentiai-energy surface for diatomic nolecule $A_{2}$ approaching a solid surface with $z$ the center-of-mass distance from the surface and o the intra-molecular separation relative to the neutral molecule equilibrium distance. The entrance $(z \rightarrow \infty)$ and exit $(p \rightarrow \infty)$ channels correspond to an intact molecule ( $\left.A_{2}\right)$ and 2 adsorbed particles $\left(2 A_{a d s}\right)$, respectively, and are separated by a negative molecular ion resonance $\left(\mathrm{A}_{2}{ }^{-}\right)$. Transitions between the various states can occur along the "seam" where the potential energies of adjacent states are equal.

$\mathrm{A}_{2}$ (gas) $\rightarrow \mathrm{A}_{2}^{-}$(nondissoc. ads.) $\rightarrow \mathrm{A}_{2}{ }^{*}$ (gas)
$\mathrm{A}_{2}$ (gas) $\rightarrow \mathrm{A}_{2}$ (nondissoc. ads.)
$\mathrm{A}_{2}$ (gas) $\rightarrow \mathrm{A}_{2}$ (non dissoc. ads.) $\rightarrow 2 \mathrm{~A}$ (dissoc. chemisorbed)

We have studied the activated dissociative chemisorption of a diatomic molecule for the case where an activation barrier exists in the exit channel along the seam (curve crossing) separating the $A_{2}$ - resonance from the dissociated state. 
Due to the fact that the incident $A_{2}$ molecule first goes through the $\mathrm{A}_{2}{ }^{-}$resonance, memory of the initial partitioning of energy (normal versus parallel translational, vibrational, etc.) is erased. Consequently, activated adsorption could depend upon the total energy available, not just the normal translational component, as recently observed experimentally. This conclusion runs counter to all previcus surface-science intuitive models based on one-dimensional potential-energy curves, thus demonstrating the utility of new conceptual models. We have calculated mean translational-to-vibrational energy redistributions, dissociative sticking probabilities, and have included the role of substrate viscous forces and mode mixing. We are in the process of establishing the role of chaotic versus quasi-periodic intra-molecular dynamics on surface processes.

Charge-transfer processes in molecule-surface interactions constitute a large class of events which are significant in determining chemical outcomes. From the point of view of quantum intra-molecular dynamics, charge transfer (harpooning) between a surface and an incident molecule is equivalent to potential surface hopping in which the wavepacket representing the initial state of a particular molecular degree of freedom is instantly placed at a non-equilibrium location on a new potential surface. By following the time evolution of the wavepacket, observable dynamic properties relevent both to spectroscopy and chemistry can be calculated, often in a significantly more economic way than by using traditional stationary-state techniques and almost always in a physical more transparent manner.

Adapting the wavepacket-dynamics approach developed previously by Heller to surface dynamics, we have constructed a wavepacket model for describing intra-molecular dynamics in surface scattering processes involving charge transfer. The essence of the model is shown in Fig. 5 where intra-molecular potential curves for a neutral diatomic molecule and a negative molecule ion are shown. The center-of-mass translational motion is described by some imposed surface scattering trajectory which sets the time interval $\tau_{R}$. Initially the molecule $A_{2}$ is in its vibrational ground state. At time $t=0$, an electron transfers from the substrate to the molecule, the $\mathrm{A}_{2}{ }^{-}$curve is switched on, and the wavepacket propagates on the new curve until time $t=\tau_{R}$, when the electron returns to the solid. This event has the effect of switching back on the $A_{2}$ potential and allowing the now-moving wavepacket to propagate on the neutral curve. We have developed a quantitative theory for the vibrational excitation distribution in the scattered molecule in terms of the molecular parameters and the iffetime of the $\mathrm{A}_{2}{ }^{-}$intermediate state. A typical set of vibrational distributions is shown in Fig. 6 for bromine as a function of $n$, the vibrational quantum number, and with the intermediate state lifetime treated parametrically. Not only is substantial excitation experienced, which varies with $\tau_{R}$, but also significant dissociation can occur due to curve hopping. The wavepacket dynamics serves as a complement to the classical studies and holds promise for being fairly easily zeneralized to polyatomic molecules with several degrees of freedom. Work is in progress to extend these studies to more complicated systems, and into the area of stimulated desorption. 


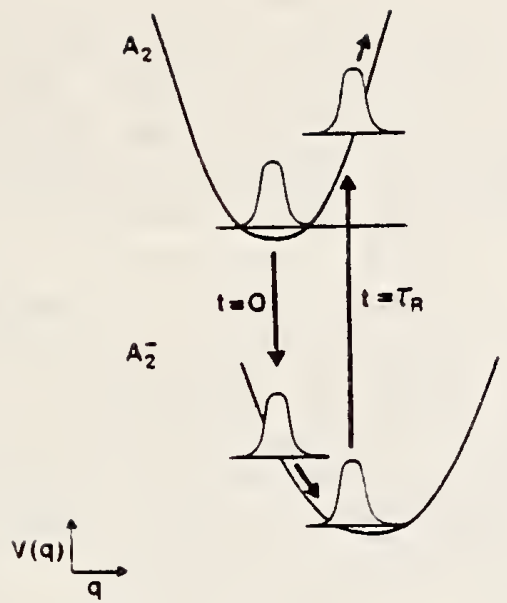

Fig. 5 Intramolecular potential energy curves illustrating the wave-packet propagation of the relative nuclear coordinates associated with the existence of a negative-molecular ion within the time interval $0<t<\tau_{R}$. The return of the wave packet onto the $A_{2}$ curve at $t=\tau_{R}$, while possessing both kinetic and potential energy, is responsible for vibrational excitation of the final state of the $\mathrm{A}_{2}$ molecule.

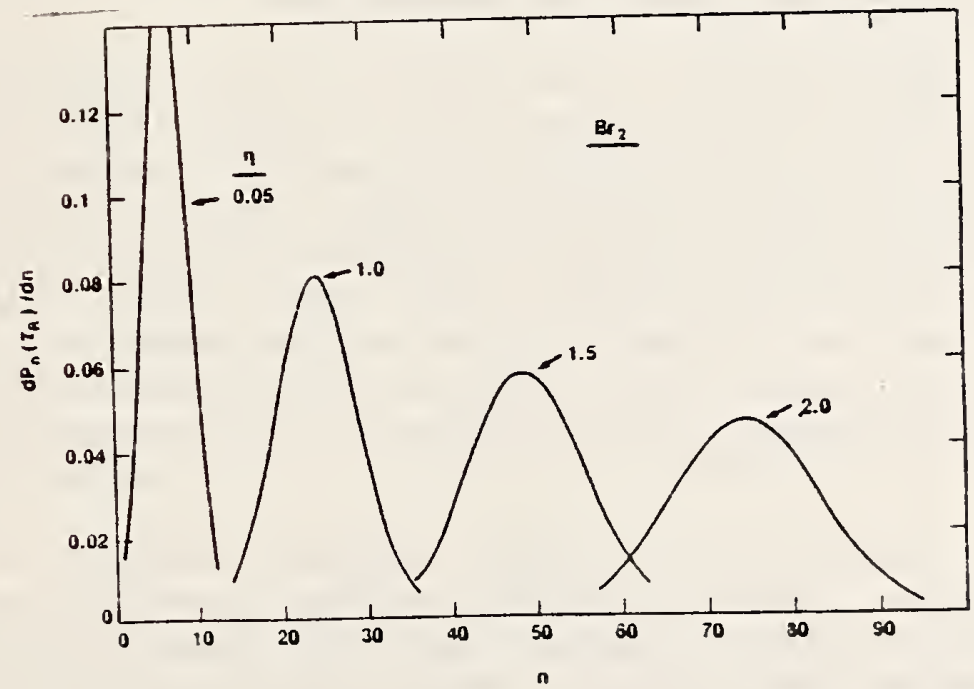

Fiz. 6 Predicted vibrational excitation distributions of scattered molecules as a function of vibrational quantum number for tromine molecules which have experienced the sequence of curve hopping 3 shown in Fig. 5. The resonance lifetime $\tau_{R}$ (via $\eta=\tilde{\omega} \tau_{R}$ with $\bar{w}$ the $\mathrm{Br}_{2}{ }^{-}$frequency) is treated parametrically. The curve labeled $n=2$ corresponds to an internal vibrational population distribution of the oromine molecule containing $1 \mathrm{eV}$ total vibrational energy. 
Vibrational spectroscopy of molecules adsorbed on solid surface is one of the dominant tools for obtaining microscopic chemical information of chemisorption systems. While the principal use has been through interpretation of the energy position of a loss feature, increasing attention has been focused on the information content of the lineshape and width. Aside from inhomogeneous broadening effects, the surface science community has, almost without exception, assumed that the linewidth is a direct measure of inelastic damping of an excited oscillator outside a surface. Many debates on the relative roles of phonon versus electronhole pair contributions to dissipation have relied on the characteristics of the observed linewidth as the final determinant. Although elastic dephasing collisions are well known in other vibrational spectroscopies to usually be the dominant source of observed linewidths in systems involving condensed phases, their importance in surface vibrational spectroscopy has not been recognized. In collaboration with $\mathrm{Dr}$. A. Luntz of the IBM Research Laboratory, San Jose, we have developed a theory based on the exchange-coupling dephasing model due to Harris, in which there is anharmonic coupling between intra-molecular $\left(-2000 \mathrm{~cm}^{-1}\right)$ modes and largeamplitude, low-frequency modes associated with bonding to the surface. The latter modes, coupled to the stochastically fluctuating phonon both of the substrate, are responsible for dephasing collisions that can yield large $\left(-10-50 \mathrm{~cm}^{-1}\right)$ vibrational linewidths. The predicted temperature dependence of the linewidth is in accord with very recent experimentally measured values, adding strong support to our theory. From this work we conclude that the vibrational linewidths of intra-molecular adsorbates modes cannot generally be related to dissipative effects, as has been assumed previously, without independent measurements which distinguish between dissipative and dephasing broadening mechanisms.

We plan to continue work in the area of surface reaction dynamics with near-term emphasis on the determination of self-consistent reaction trajectories, realistic electron-hole pair coupling constants, determination of energy-redistribution patterns amongst translational, vibrational, rotational, and electronic degrees of freedom of "reactant" molecules and electron and phonon modes of surfaces under controllable dymamic and reactive conditions, and synthesis of the various components of the elementary reaction theories into theories of experimentally realizable processes. Special emphasis will be placed on the phenomenon of dissociative surface processes and on the role of non-linear dynamics.

In addition, analysis will be made, whenever possible, of novel experimental results. Current interest includes laser-assisted surface processes and state-to-state analysis, high-resolution surface vibrational spectroscopy, neutron spectroscopy of metal hydrides, and other experimental probes of non-adiabatic effects. 
G. Fabrication of Fluorescent Screens

(A. J. Melmed)

Fluorescent phosphor screens are widely used in the Surface Science Division and in other parts of NBS, and very often the phosphor screen must be custom-made. The preparation process usually requires two or more days, especially if a high-quality, uniform phosphor deposition is required.

In collaboration with Dr. O. Frank of the Fritz-Haber Institute, Berlin, a simple method was developed for preparing fluorescent screens on 3lass or metal substrates. Whereas the former method required a careful balance of large volumes of complex chemical solutions for maintaining proper ion and binder concentrations in the phosphor settling solution, the new process replaces the chemistry with rapid mechanical steps and smaller volumes of liquid. The entire process can be completed in about three hours. 


\section{A. Introduction}

The Surface Science Competence Program is a part of the NBS "Competence Program." The NBS program was introduced to provide long-term support for the establishment and maintenance of areas of excellence in science and technology contributing to the NBS mission and the projected needs of the NBS clientele.

The Surface Science Competence Program NBS Task 26103 was established with new staff $51 / 2$ years ago. Initial plans envisaged new research activities in three areas: (a) chemical dynamics on surfaces, (b) ion interactions with surfaces, and (c) surface properties of novel materials; there are, however, appreciable overlaps among these areas with other Division projects. Specific projects have been selected based on a judgement of their scientific merit and on assessments of the future scientific and technical challenges requiring NBS expertise and action. Our strategy has been to involve existing Division staff in the new activities to the maximum extent possible and to implement both new experimental and new theoretical activities.

Section 4.B contains reports of the technical activities initiated as part of the Surface Science Competence Program. Section 4.C contains reports of activities by Surface Science Division staff concerned wi th the development of experiments at the Brookhaven National Synchroton Light Source. These experiments are being conducted as part of NBS Task 26105 which was initiated in 1980.

B. Reports of Surface Science Competence Program Activities

1. ESDIAD-EELS Studies of the Structure and Reactivity of Adsorbed Species (N. D. Shinn, T. E. Madey)

The determination of the local bonding geometry of adsorbed species on surfaces is of fundamental importance to the development of a conceptual basis of surface chemistry. Fundamental studies of adsorbate structures on single crystals are just beginning to give baseline information to technologies such as heterogeneous catalysis, corrosion prevention, electrochemistry, and semiconductor design.

We have constructed an unique apparatus combining ESDIAD (Electron Stinulated Desorption-Ion Angular Distributions) and EELS (Electron Energy-Loss Spectroscopy). These techniques in combination make a powerful facility for surface structural characterization.

This year high resolution electron energy loss spectroscopy (EELS), electron stimulated desorption ion angular distributions (ESDIAD), low energy electron diffraction (LEED) and Auger electron spectroscopy (AES) have been used to identify two distinct, sequentially populated, molecular CO binding states at $120 \mathrm{~K}$ on $\mathrm{Cr}(110)$. The first is a new molecular state 
$\left(a_{1}\right)$ with a substantially weakened $C O$ bond, believed to be a precursor state to dissociation, whereas the second state $\left(\alpha_{2}\right)$ is comparable to terminally bonded $\mathrm{CO}$ on other transition metals. The CO stretching prequencies of the $\alpha_{1}$ state $\left(-1150-1330 \mathrm{~cm}^{-1}\right)$ are substantially shifted from the gas phase $\left(2143 \mathrm{~cm}^{-1}\right)$, and are believed to be the lowest values ever reported for adsorbed molecular $\mathrm{CO}$ on any clean or promoted transition-metal surface.

Initially, co adsorbs with a sticking probability of -0.9 which decreases upon formation of a $c(4 \times 2)$ CO overlayer. EELS spectra reveal a weak $450 \mathrm{~cm}^{-1}$ loss and, surprisingly, two strong features at 1150 and 1330 $\mathrm{cm}^{-1}$. All losses increase with additional co exposure, shifting to - 475 , 1200, and $1330 \mathrm{~cm}^{-1}$ at full $\mathrm{c}(4 \times 2) \mathrm{CO}$ overlayer formation. With further CO exposure, the $c(4 \times 2)$ LEED pattern reverts to $(1 \times 1)$ symmetry with high background intensity, and a new triplet of EELS loss features appears at 495, $1865,1975 \mathrm{~cm}^{-1}$. ESDIAD measurements as a function of co exposure show atypically weak $\mathrm{O}^{+}$ion emission along [110], increasing in intensity only after the appearance of the latter triplet of EELS features.

These data are suggestive of $\alpha_{1}$ CO molecules oriented nearly parallel to the $\operatorname{Cr}(110)$ surface, while $\alpha_{2}$ CO molecules are oriented normal to the surface at ei ther atop or bridge-bonded sites. Figure 7 illustrates representative EELS spectra for the sequentiaI $\alpha_{9}, \alpha_{2}$.CO population experiment. Further investigation of the unique $\alpha_{1}$, co state and the decomposition of both $\alpha_{1}$ and $\alpha_{2}$ co are planned.

Also this year, an EELS/ESDIAD/LEED (low-energy electron diffraction) study of $\mathrm{O}_{2}$ adsorption on $\mathrm{Cr}(110)$ has found an unexpected molecular adsorption state below 200K. As seen in figure 8 , the vibrational spectrum at $120 \mathrm{~K}$ includes Iosses at $615 \mathrm{~cm}^{-1}$ (due to atomic oxygen) and $1020 \mathrm{~cm}^{-1}$, the latter being attributed to the $0-0$ stretch of $\mathrm{O}_{2}$ (ads). At $300 \mathrm{~K}$, only atomic oxygen remains with a characteristic loss at $605 \mathrm{~cm}^{-1}$. Low oxygen exposures at $300 \mathrm{~K}$ result in the formation of a $\mathrm{p}(4 \times 2) 0$ overlayer and the observation of a $290 \mathrm{~cm}^{-1}$ loss feature, possibly due to a surface phonon of the ordered overlayer.

ESDIAD measurements of the low temperature adsorption have identified six azimuthally ordered cones of $\mathrm{O}^{+}$ion emission away from the surface normal. These correlate with the appearance of the 0-0 vibrational loss, indicating that there are six discrete tilted orientations for $\mathrm{O}_{2}$ (ads), reflecting the pseudo-hexagonal symmetry of the $\operatorname{Cr}(110)$ surface. In contrast, atomic oxygen results in a single [110] directed cone of $0^{+}$ emission observable at all temperatures.

In addition, an example of co-adsorbate interactions inducing a significant molecular reorientation has been found in $\mathrm{CO} / \mathrm{O}_{2}$ titration experiments on $\mathrm{Cr}(110)$. $\mathrm{Cr}(11)$ pre-dosed with atonic oxygen to form a $\mathrm{p}(4 \times 2)$ structure completely blocks the $\alpha_{1}$ CO adsorption state. Only the 495, 1865, and $19 \overline{75 \mathrm{~cm}^{-1} \text { CO }}$ vibrational features are seen, in addition to the $\mathrm{Cr}-0$ stretch at $605 \mathrm{~cm}^{-1}$. A more dramatic result is found when a $c(4 \times 2)$ Co adlayer - i.e., the saturation coverage of the $\alpha_{1}$ binding state - is subsequently exposed to oxygen. The stepwise increase in suriace 


\section{$\operatorname{Cr}(110) / C O$}

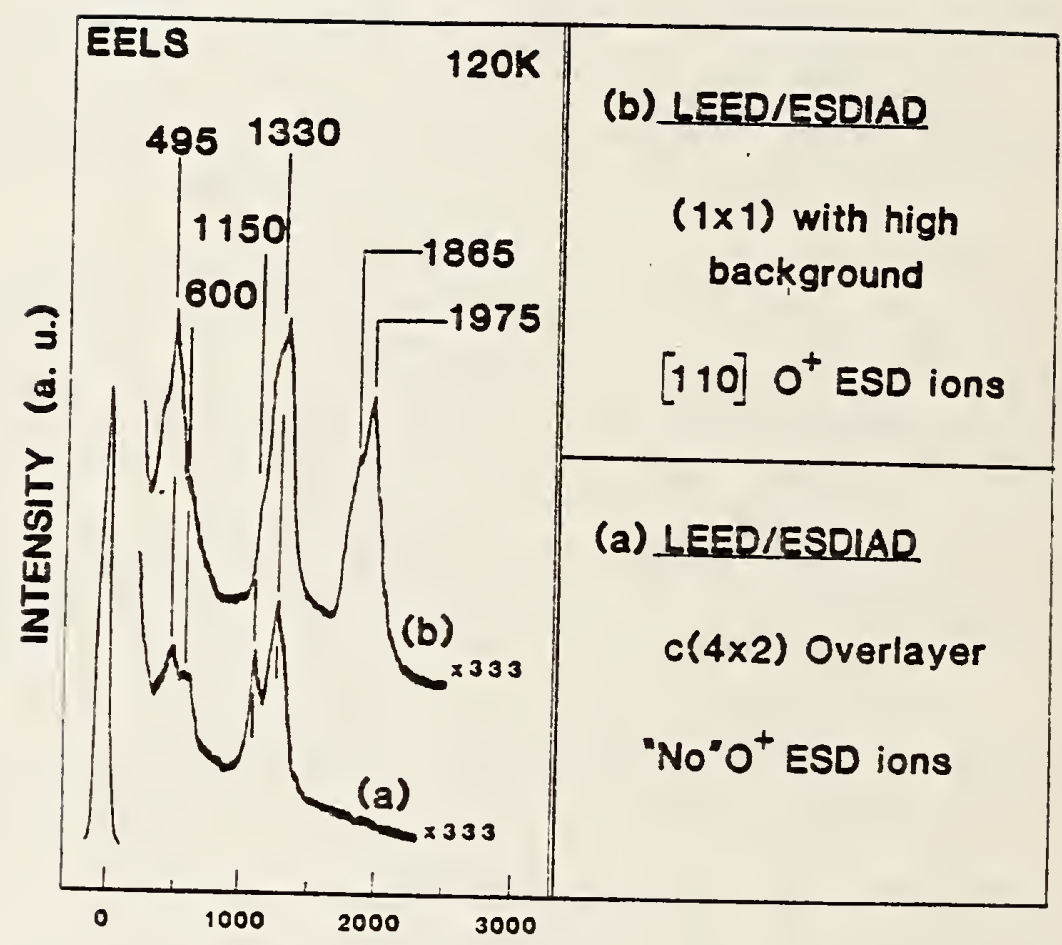

ENERGY LOSS $\left(\mathrm{cm}^{-1}\right)$

Eig. 7. Summary of EELS/ESDIAD/LEED data for CO adsorption on Cr(110) at $120 \mathrm{~K}$. Panel at left shows representative spectra for two stages of chemisorption. $\alpha_{1}$ CO is characterized by spectrum (a) with CO stretching frequencies at 1150 and $1330 \mathrm{~cm}^{-1}$, as well as a $\mathrm{Cr}-\mathrm{CO}$ mode at $-475 \mathrm{~cm}^{-1}$ and a residual $\mathrm{cr}-0$ stretch at $600 \mathrm{~cm}^{-1}$. Further CO exposure populates the $\alpha_{2}$ CO state with corresponding, site-specific CO stretching frequencies at 1865 and $1975 \mathrm{~cm}^{-1}$. The Cr-CO stretching mode is observed at $495 \mathrm{~cm}^{-1}$. 


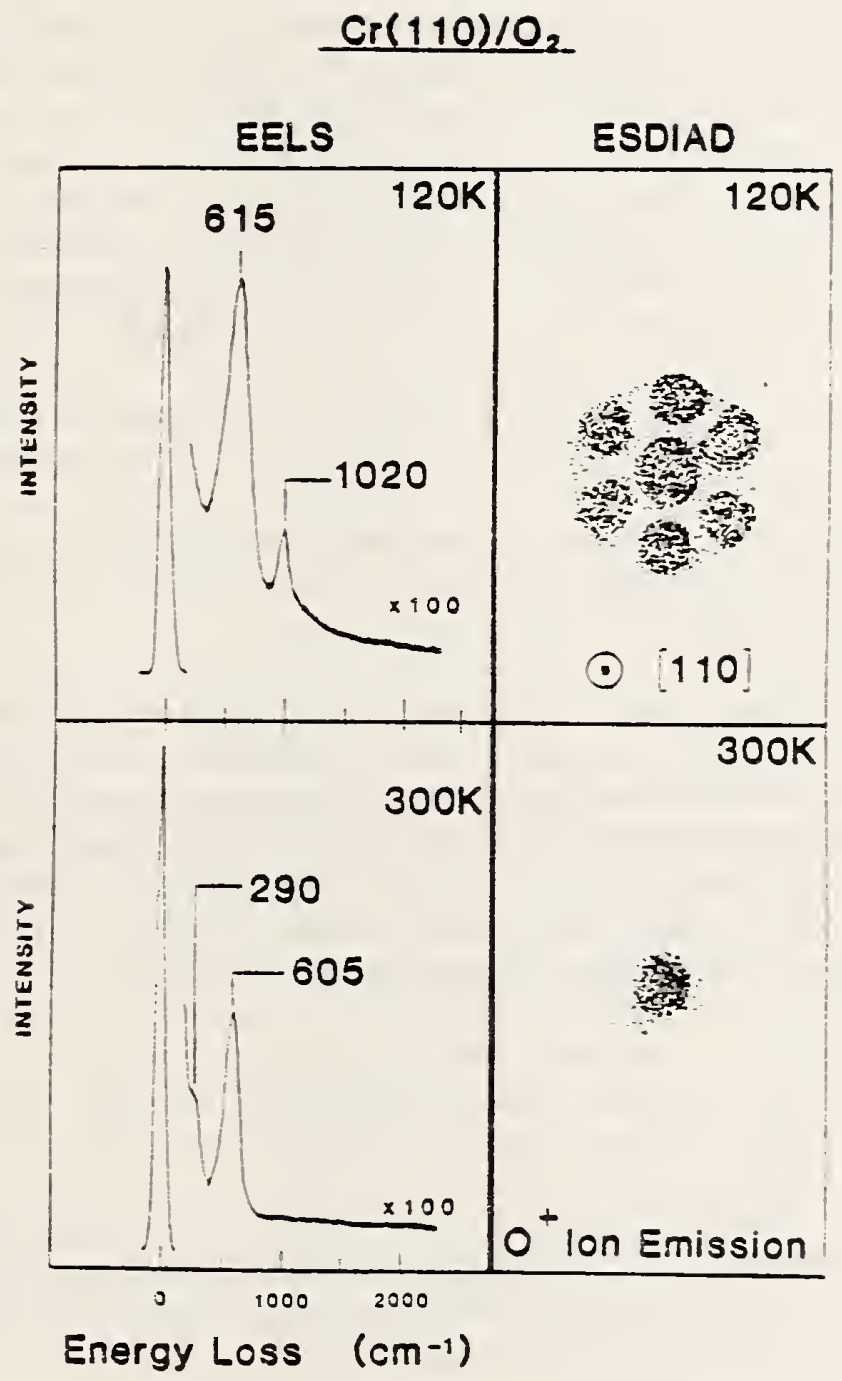

Fig. 8 Vibrational spectra for oxygen chemisorption on Cr(110) with corresponding ESDIAD patterns. At $300 \mathrm{~K}$, atomic oxyzen results in a single [110] directed $\mathrm{O}^{+}$ion beam and a Cr-0 szretching erequency of $605 \mathrm{~cm}^{-1}$. The $2909 \mathrm{~cm}^{-1}$ shoulder is observable only at low oxygen coverages. Low-temperature adsorption populates a new molecular state, with 0-0 stretching frequency of $1020 \mathrm{~cm}^{-1}$ and $\mathrm{s} 1 \mathrm{x}$ new off-normal $0^{+}$ion beams. 
oxygen causes the $1200-1300 \mathrm{~cm}^{-1}$ Co intensity to gradually decrease while a commensurate increase is seen in loss features at $-470, \overline{1865 \text { and }} 1975$ $\mathrm{cm}^{-1}$. This oxygen-induced conversion of $\mathrm{CO}$ from the first binding state to the second (terminally bound) state further demonstrates the existence of two molecular $c 0$ states at $120 \mathrm{~K}$. Detailed coverage-dependent studies are underway to elucidate these apparent site-blocking and site-competition effects.

We plan to continue of the $\operatorname{Cr}(110)$ chemisorption studies with particular attention to the effects of steps, sub-suriace oxide, and contaminants. Further investigation of the complex interaction of $\mathrm{CO}$ and $\mathrm{O}_{2}$ is necessary to confirm evolving models for this system. Additional experiments with $\mathrm{N}_{2}, \mathrm{CO}_{2}$, and $\mathrm{H}_{2} \mathrm{~S}$ are anticipated to assess the chemical activity of $\mathrm{Cr}(110)$ for dissociative adsorption and the role of common bulk impurities $(\mathrm{N}, \mathrm{S})$ in adsorbate ordering. The investigation of $\mathrm{Cr}(100)$ surface chemistry and adsorbate-induced surface reconstruction continues. In view of the surprising results of the $\mathrm{Cr}(110) / \mathrm{CO} / \mathrm{O}_{2}$ investigation, comparable experiments are planned for the less dense $\mathrm{Cr}(100)$ surface in order to address the question of site-specific $C O$ chemisorption modes on chromium. To date, the only work on molecular adsorption on singlecrystal chromium surfaces is that done in this laboratory, although there is a wider interest in assessing chemisorption trends in the family of $\mathrm{Cr}$, Mo, and $W$ transition metals.

We plan to interface the high-resolution EELS spectrometer to an LSI 11 microcomputer. This will enable semi-automated data acquisition in a pulse-counting mode as well as facilitated and expanded data-manipulation capabilities. These improvements to the EELS instrument are necessary for the satisfactory performance of relevant, low-signal experiments. Initial experiments planned will probe the low coverage interactions of $\mathrm{H}_{2} \mathrm{O}$ and $O$ (ads) on $\mathrm{Ag}(110)$ and also $\mathrm{NH}_{3}$ on oxygen pre-dosed $\mathrm{Ni}(110)$. In both cases, vibrational data is needed to confirm the models developed in previous ESDIAD/LEED/TDS (thermal desorption spectroscopy) studies for the respective hdyrogen abstraction reactions $\mathrm{H}_{2} \mathrm{O}+\mathrm{O}$ (ads) $\rightarrow 2 \mathrm{OH}(\mathrm{ads}$ ) (on $\mathrm{Ag}(110))$ and $\mathrm{NH}_{3}+\mathrm{O}(\mathrm{ads}) \rightarrow \mathrm{NH}_{2}(\mathrm{ads})+\mathrm{OH}(\mathrm{ads})$ (on $\mathrm{Ni}(110)$.

We will complete the fabrication, testing, and incorporation of the "high pressure" reaction chamber and transfer mechanism to the EELS/ESDIAD system. This powerful combination of ultrahigh vacuum spectroscopic techniques and actual kinetic measurements

on the same model catalyst will allow the correlation of reaction rates to models derived irom detailed chemisorption studies. Of particular interest is the hydrogenation of Co over the atomically corrugated $\mathrm{Ni}(110)$ and $\mathrm{Ni}(311)$ surfaces. Although recent studies of other structured model catalysts have revealed interesting chemisorption results, the significance of these results has not been established. Our studies will directly test the catalytic importance of an atomically rough surface as well as expand our knowledge of chemisorption on "defective" surfaces. 
2. Laser Diagnostics of Surface Dynamics

(R. R. Cavanagh)

The pathways and rates of energy transfer at surfaces are not readily accessible with conventional surface-characterization techniques. A variety of laser-based techniques, however, have been applied to similar questions of molecular dynamics in both the gaseous and liquid states. In this program, the focus is on extending established laser methods and developing new laser-based techniques for addressing the issue of energy transfer at surfaces. The program includes exploitation of both the spectral and temporal resolutions available in state-of-the-art lasers.

Two types of experiments are underway in which laser diagnostics are employed to probe dynamical processes at surfaces. This work is performed in collaboration with Drs. King, Stephenson, Mantell, Heilweil, and Casassa of the NBS Molecular Spectroscopy Division.

\section{a. Laser-Excited Eluorescence as a Probe of Surface Dynamics}

Molecular desorption from surfaces is representive of chemical conversion processes which transform reactants (adsorbed molecules on surfaces) to products (gas-phase molecules and bare surfaces). In order to characterize and utilize such reaction processes, it is essential to have state-specific diagnostic probes suitable for such measurements. The focus of this work is to explore the appiication of laser-based probes (such as laser-excited fluorescence and multiphoton ionization) to adsorbate and desorption processes. Processes such as thermally activated desorption, chemically induced desorption, laserinduced desorption, and electron-stimulated desorption exemplify the scope of the project. This work is being performed collaboratively with Drs. King and Mantell.

A high-resolution excimer-pumped tuneable dye laser is being used to study thermal desorption in a new ultrahigh vacuum chamber. The laser operates under microprocessor control, giving single-mode turability over $0.5 \mathrm{~cm}^{-1}$, at a resolution of $0.005 \mathrm{~cm}^{-1}$, or over $20 \mathrm{~cm}^{-1}$ at a resolution of $0.03 \mathrm{~cm}^{-1}$. The output energy is typically $100 \mu \mathrm{J}$ at $226 \mathrm{~nm}$. Combining the present tuning range of the laser (800-215 nm) with the improved surface diagnostics in the vacuum chamber, a much broader range of desorption diagnostics is possible.

Experiments to probe the spatial orientation of the No molecular axis following thermal desorption from $\mathrm{Ru}(001)$ have been performed. Since inhomogeneous magnetic fields on the order of 10 mGauss are suficient to perturb the measurements, the magnetic shielding in the new chamber was critical, as was the ability to take data only at the zero crossing of the current used to heat the Ru crystal. We have not found any preferred alignment (i.e., the molecular axis is neither predominantly perpendicular nor parallel to the crystal plane). 
The influence of adsorbate-adsorbate interactions on the dynamics of thermal desorption is essentially unexplored. Our current sensitivity level of -5 percent of a monolayer will permit us to examine the magnitude of such effects. In addition during the coming year thermal desorption from substrates which are inactive for NO decompostion (such as $\mathrm{Pt}(111)$ or $\mathrm{Pd}(111)$ ) will be characterized. In such systems (where dissociative channels are not in competition with thermal desorption), strong polarization effects are anticipated. Laser surface heating will be explored. Heating pulses of 10 ns duration will be used to initiate molecular desorption. The resulting internal-state distribution will provide an unique characterization of the heat bath sampled during the desorption process. Also, the extension of state-specific diagnostics to molecules such as carbon monoxide or hydrogen will be initiated with the construction of the appropriate four-wave mixing facilities for working in the $150 \mathrm{~nm}$ wavelength region.

\section{b. Real-Time Measurements of Energy Transfer at Surfaces}

The time scale for energy transfer at surfaces remains a major uncharacterized parameter of chemistry at interfaces. The application of time-resolved laser techniques to a variety of solid surface problems holds promise for providing real-time diagnostic of relaxation mechanisms. With a variety of techniques, the surface response to laser excitation (using infrared, visible, or ultraviolet pulses) is probed in real time.

In collaboration with Drs. Stephenson, Heilweil, and Casassa, the iirst real-time measurements of the vibrational relaxation of a surface species have been made. The vibrational relaxation of $\mathrm{OH}\left(3690 \mathrm{~cm}^{-1}\right)$ groups on colloidal silica ( -120 A diameter particles, $\left.200 \mathrm{~m}^{2} / \mathrm{gm}\right)$ dispersed in carbon tetrachloride has been found to be $150 \mathrm{ps}$. This relaxation time is forty times shorter than for comparable diatomic oscillators at this wavelength, yet the time is three orders of magnitude longer than that obtained from linewidth arguments. The implications of the observed lifetime will only be fully realized when a variety of systems with different chemical properties have been explored. It is already apparent, however, that in this system vibrational relaxation is slow on the time scale of rotational reorientation.

Infrared pump-probe experiments are a zeneral method for establishing rates and mechanisms of energy transfer. Three issues will be addressed rext. First, the measurement technique will be extended to pressed discs in vacuum to determine why the vibrational relaxation at the solid-liquid interface is substantially different from that at the solid-vacuum interface. Second, the relaxation rates for adsorbed $O H$ species on other insulator surfaces such as $\mathrm{Al}_{2} \mathrm{O}_{3}, \mathrm{ZnO}$, and zeolites will be examined to determine the correlation between vibraticnal relaxation and substrate. Third, two-color pump-probe techniques will be applied to establish the pathways of viorational relaxation on surfaces. 
3. Neutron Inelastic Scattering by Adsorbed Species on Surfaces (R. R. Cavanagh, R. D. Kelley, T. J. Udovic)

Neutron scattering is regarded generally as a tool of solid state physicists. Recently, however, there have been increasing neutron scattering efforts in the areas of material and chemical sciences. At NBS, the Surface Science Division has been working with the Reactor Radiation Division to apply neutron scattering to molecular processes at surfaces. Our efforts in the past year can be divided into several categories.

The Neutron Inelastic Scattering, (NIS) spectra have been measured for the first time during a steady-state catalytic chemical reaction. In this case the hydrogenation of $C O$ to hydrocarbons. The "in situ" measurements of adsorbate vibrations have been made using a Raney nickel catalyst (a high surface area material). Reaction products were measured with gas chromatography while the vibrational spectra were being recorded. Measurements were made as a function of temperature and $\mathrm{H}_{2} / \mathrm{CO}$ ratio. Analysis of the spectral changes which accompany changes in reaction parameters is not complete. However, it is quite clear that the introduction of $C O$ into the hydrogen gas stream results in a substantial reduction in the concentration of atomic hydrogen on the nickel surface. Vibrational modes due to hydrocarbon species are also detected. The intensity of these modes varies in a complex way with changes in reaction conditions (partial pressure of carbon monoxide, temperature, and flow rate)..

NIS from adsorbed species on platinum black is being investigated. The hydrogen spectra are being analyzed and the chemical activity of "defect" hydrogen is being probed. Present data permits the identification of $\mathrm{H}_{2} \mathrm{O}$ formation at room temperature due to reaction of oxygen with preadsorbed hydrogen. In addition, NIS of $\mathrm{NH}_{3}$ adsorbed on such a "defect" surface exhibits vibrational spectra in distinct contrast to that anticipated for close-packed platinum planes.

The NIS spectra of the chemisorption of $\mathrm{C}_{2} \mathrm{H}_{2}$ and $\mathrm{C}_{2} \mathrm{H}_{4}$ on Raney nickeI were analyzed. Spectra were taken as a function of surface coverage, of adsorption temperature, and of subsequent heat treatment. These data indicate that at low temperatures $\mathrm{C}_{2} \mathrm{H}_{2}$ adsorbs molecularly on the nickel surface. In the case of ethylene adsorption, some bond dissociation takes place even at $120 \mathrm{~K}$ leading to a mixed adsorbed phase composed predominantly of a molecular $\mathrm{C}_{2} \mathrm{H}_{4}$ species. The vibrational spectra of the molecularly adsorbed species are very similar to those observed on flat $\mathrm{Ni}(111)$ zingle crystal surfaces with electron energy-ioss spectroscopy. Thus, as was found on high surface area platinum in an earlier Nis study, steps and other crystal defects (which are likely to be present in a heterogeneous material like Raney nickel), have little influence on the structure of the molecularly adsorbed hdyrocarbon. However, the presence of crystal defects can account, by aralogy with single-crystal work, for the low-temperature $\left(\mathrm{C}_{2} \mathrm{H}_{2}, \mathrm{I}>200 \mathrm{~K} ; \mathrm{C}_{2} \mathrm{H}_{4}, \mathrm{~T}>120 \mathrm{~K}\right)$ decomposition of the molecularly adsorbed species on the Raney nickel surface. 
NIS experiments have been initiated on a variety of new materials including ruthenium black and zeolite H-rho. Novel behavior of hydrogen has been observed in each case. In the zeolite, for example, a pronounced reversible conversion of bound hydrogen is apparent when the sample is heated from $275 \mathrm{~K}$ to $550 \mathrm{~K}$. The removal of a peak at $1060 \mathrm{~cm}^{-1}$ is accompanied by an increase in the intensity of a peak near $200 \mathrm{~cm}^{-1}$.

We plan to extend NIS adsorption studies to supported catalysts, e.8., $\mathrm{Pt} / \mathrm{Al}_{2} \mathrm{O}_{3}$ or $\mathrm{Pt}$ in zeolite $\mathrm{Y}$. We will examine the changes in the vibrations of chemisorbed hydrogen with particle size. The supported catalysts will be prepared and characterized in collaboration with various external laboratories. Further analys is of the material will be provided by neutron diffraction and possibly small-angle neutron scattering. We will continue the "in-situ" reaction studies of $\mathrm{H}_{2} / \mathrm{CO}$ reactions on the nickel catalyst. Both increased and decreased reaction temperatures and increased partial pressure of $\mathrm{CO}$ are obvious reaction-parameter changes which could clarify the nature of the surface species which we have detected on the catalyst surface during reaction.

Next year, we will examine the adsorption of hydrogen on Pd black. Neutron scattering can distinguish between chemisorbed hydrogen and hydrogen absorbed into the bulk. We will study the vibrational spectroscopy of hydrogen on the surface and the conversion to the bulk hydride. NIS will be utilized to probe the surface chemical behavior of RuS 2 a potentially promising hydrodesulferization catalyst. The adsorptive behavior of $\mathrm{H}_{2}$ and $\mathrm{H}_{2} \mathrm{~S}$ on both Ru black and sulfided Ru black will be further investigated. Future plans involve investigations the adsorption of thiophene on $\mathrm{RuS}_{2}$ in an attempt to elucidate the adsorbate-surface geometry. Knowledge of the exact adsorption scheme (i.e., o-bonded "edgewise adsorption" through the sulfur heteroatom vs. m-bonded "flatwise adsorption" through the aromatic $\pi$-cloud) provides insights concerning the type of reaction favored (i.e., hdyrogenolysis vs. hydrogenation) during hydrodesulfurization.

Further studies of adsorbate diffusion will be facilitated with the installation of a cold neutron source on the NBS reactor during the rall of 1984. Coupled with a redesigned time-of-flight spectrometer, the capability to probe a wider range of momentum transfers will be significantly enhanced. The increased beam flux, number of detector channels, and the simultaneous reduction of constraints on sample size will all increase the flexibility of the technique.

4. Photon-Stimulated Desorption and Ultraviolet Photoemission Spectroscopy Using Synchrotron Radiation

(R. Stockbauer, R. L. Kurtz, T. E. Madey)

A major goal of this effort is to identify the electronic excitations which result in the photon-stimulated desorption (PSD) of ions from molecules adsorbed on surfaces as well as from condensed films. When possible, PSD ion jields are correlated with ultraviolet photoemission spectroscopy (UPS) of both valence states and core-electronic states of the adsorbed species. These studies provide tests of recent theoretical 
models of electron- and photon-stimulated desorption of ions. In addition, the synchrotron radiation techniques (PSD, UPS) are combined with other surface spectroscopies to study adsorption and reactions on solid surpaces.

Several "firsts" were achieved in FY 1984: the first studies of a single-crystal stoichiometric oxide surface $\left(\mathrm{TiO}_{2}(110)\right)$ using synchrotron radiation, the identification of a new ion-desorption mechanism in oxidized $\mathrm{Cr}(110)$, and the design of a new two-dimensional display analyzer for angle-resolved PSD.

During the past year, we have used synchrotron radiation to make detailed studies of the electronic excitation spectra of the oxidized $\mathrm{Cr}(110)$ surface above the $3 \mathrm{p}$ ionization threshold ( $242 \mathrm{eV}$ ). We have correlated these measurements with PSD of $0^{+}$ions, and have found a new ion-desorption mechanism: only certain core-hole states initiate ion desorption. These findings have important implications for the theory of PSD. We were the first to apply synchrotron radiation to characterize the electronic structure of a single-crystal metal oxide surface, $\mathrm{TiO}_{2}(110)$. Preliminary photoemission results have clarified the d-band contributions to the oxide valence band, and have indicated the presence of a shake-up resonance - $19 \mathrm{eV}$ below the Fermi level. We also made the first PSD study of well-characterized $\mathrm{TiO}_{2}$ single-crystal surfaces having the (110) orientation. This major test of the Knotek-Feibelman model of ion desorption reveals shortcomings in the basic theory. The studies involve "perfect" $\mathrm{TiO}_{2}$ surfaces, and "defect" surfaces produced by $\mathrm{Ar}^{+}$sputtering and indicate the importance of local-site geometry in PSD and electronstimulated desorption.

This year we installed and tested a new high-flux grazing incidence monochromator at SURF-II. The new monochromator proceeds a photon flux 20 to 50 times more intense than our present instrumentation in the photon energy range 25 to $80 \mathrm{eV}$. The range can be extended to $175 \mathrm{eV}$ with a new grating. We also completed the design and initiated construction of a ne'w display-type ion and electron energy analyzer. It is a bandpass analyzer which employs an ellipsoidal mirror; the design is based on an analyzer pioneered by Eastman. When complete, it will be the second such analyzer in operation in North America.

This coming year we plan to use variable wavelength UPS to determine, for the first time, the influence of oxide surface structure and surface defect concentration on the electronic properties of transition metal oxides. Nearly-perfect, annealed $\mathrm{TiO}_{2}$ and $\mathrm{Ti}_{2} \mathrm{O}_{3}$ will be compared with defective, Ar ${ }^{+}$-bombarded surfaces. Single crystals having different orientations will be studied; both thermally-stable and thermally-unstable surfaces (e.g. $\mathrm{TiO}_{2}(110)$ and $(100)$ ) will be examined.

We will complete the construction of the new angle-resolving, display-type ion and electron energy analyzer. We will employ this special analyzer to measure the surface core-level shifts in several related series of oxidized substrates, e.g., ranging from clean $T$ i to oxidized $\mathrm{Ti}$ metal, to stoichiometric $\mathrm{Ti}_{2} \mathrm{O}_{3}$ and $\mathrm{TiO}_{2}$. This wIII provide 
new insights into the local electronic environment of both cations and anions. We will employ the same analyzer to initiate the first angleresolved PSD studies on both nearly-perfect and defective oxides, as well as on molecules adsorbed on these surfaces. Surface structure inferred from these studies will be correlated with other measurements.

Next year we also plan to extend extend chemisorption measurements on single-crystal oxides to the temperature range below $300 \mathrm{~K}$. Surprisingly, there are virtually no chemisorption measurements reported at low temperatures. Our goal is the accurate determination of adsorption energetics and molecular structure for $\mathrm{CO}, \mathrm{H}_{2} \mathrm{O}, \mathrm{NH}_{3}$ on oxides.

5. Surface Chemical Physics of Single Crystals, Alloys and Ultrathin Films

(W. F. Egelhoff)

Interest in ultrathin (down to one atomic layer) metal films on the part of the surface-science community has greatly increased in the past three years as it has become clear that an understanding of such structures will contribute to solving a wide range of problems in surface science. Among these are an understanding of the differences between surface and bulk electronic structure, the changes of surface electronic structure associated with chemisorption, the basis of surface magnetism, the modification of surface-layer properties by the substrate, the relationship between electronic structure and epitaxial srowth, and the surface properties of alloys. The experimental program presently underway is using an integrated approach to study single-crystal surfaces, alloys and ultrathin flims to contribute to an understanding of all of the above problems.

This past year a new aspect of $x$-ray photoelectron spectroscopy (XPS) and Auger-electron spectroscopy (AES) of thin films was discovered that can be used to characterize the morphology of films and to provide a new approach to analyzing XPS and AES data. The phenomenon takes the form of enhanced intensities of photoelectrons or Auger electrons from an atom in the film along the internucleus axes towards neighboring atoms. This is illustrated in Fig. 9. For $2 \mathrm{ML}$ of copper on $\mathrm{Ni}(100)$, the inner $\mathrm{Cu}$ layer will have its core-level peak intensities enhanced at a polar angle of $45^{\circ}$, as shown, due to forward scattering by the top $\mathrm{Cu}$ layer. Figure 10 presents experimental data of the angular distributions of the $\mathrm{Cu} 2 \mathrm{p}_{3 / 2}$ signal as a function of the film thickness that show a strong peak at $\theta=45^{\circ}$ when the $\mathrm{Cu}$ film thickness exceeds $2 \mathrm{ML}$. The effect is more pronounced for electron energies greater than $500 \mathrm{eV}$; for example, in the angular profiles of the $\mathrm{CU}$ AES peak several peaks are observed that can be interpreted as arising from specific layers of $\mathrm{Cu}$ atoms. Numerical calculations demonstrate that this type of enhancement is not due to electron channeling (the Kikuchi effect) as has been suggested previously. 


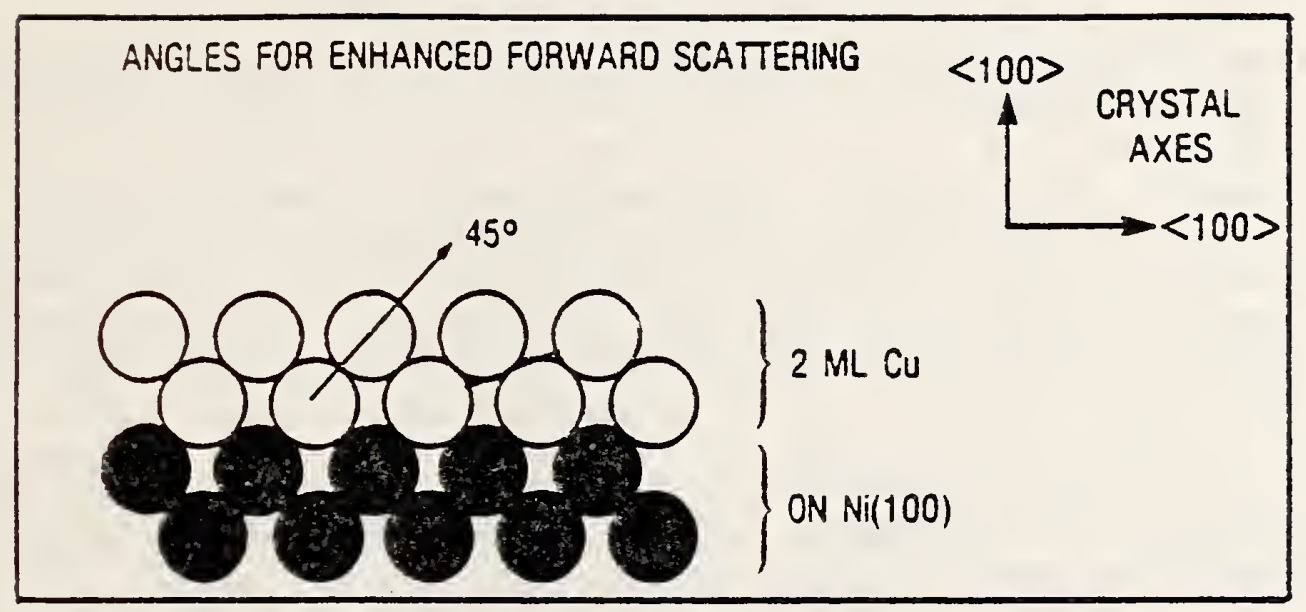

Fig. 9 Illustration of enhanced forward scattering at a polar angle of $45^{\circ}$ from the bottom $\mathrm{Cu}$ layer by the top $\mathrm{Cu}$ layer of a $2 \mathrm{ML}$ copper film on a $\mathrm{Ni}(100)$ substrate.

EPITAXIAL CU ON NI(100)

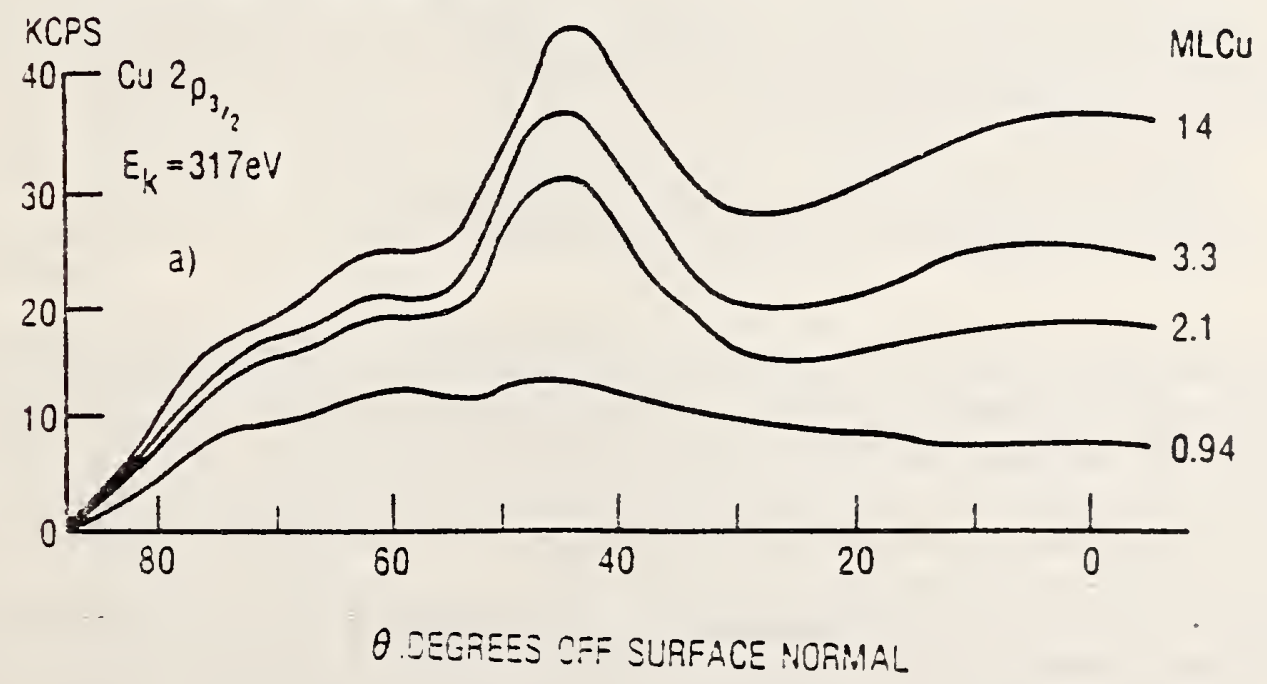

Fig. 10 Intensity of the $\mathrm{Cu} 2 \mathrm{p}_{3 / 2}$ XPS signal as a function of polar angle ior different thicknesses of copper (in monolayers) epitaxially deposited on $\mathrm{Ni}(100)$. 
This forward scattering phenomenon has been found to be an easily used, new approach to gaining structural information about surfaces. The enhanced intensities along internuclear axes provide a very useful diagnostic of whether epitaxy, agglomeration, surface alloying, or interdiffusion are occuring at a multicomponent surface.

The forward-scattering phenomenon can be utilized to provide additional, new data important for surface and interface science. The XPS signal originating from a particular layer can be selected by appropriate choice of angle. This provides a convenient means of measuring the core-level binding energies of surface atoms and comparing them with the binding energies of atoms in other layers and in the bulk. The phenomenon also provides a new way of determining the background in XPS and AES data; this method is described in Section 2.A.8. Finally, this work has shown that conventional methods of measuring electron attenuation lengths for quantitative surface analysis by XPS and AES can be subject to appreciable errors, as described in Section 2.A.7; the "effective" attenuation length for the analysis of thin ordered films will depend on the film morphology and crystalline perfection as well as the instrumental geometry.

A major, invited review article, "Core-Level Binding-Energy Shifts in Solids and at Surface," is currently being written for Surface Science Reports. In addition work will continue on the development of the forward-scattering effect as a tool for structural and electronic studies of surfaces and interfaces; the refinement of the use of the equivalent-core Born-Haber-cycle method for determining thermochemical quant:ties at surfaces and interfaces; and the initiation of a program to make nonequilibrium, exotic alloy structures such as alternating single, epitaxial atomic layers of $\mathrm{Cu} / \mathrm{Ni} / \mathrm{Cu} / \mathrm{Ni} / \mathrm{Cu}$.... This plan represents an extension of the field of ordered alloy systems to a potentially unlimited number of nonequilibrium ordered systems. Novel materials such as these have not been prepared previously but are expected to have unusual physical and chemical properties.

6. Atom-Probe Field-Ion Microscopy of Materials

(A. J. Melmed, R. Klein, M. E. Twigg)

We are completing the construction of an Atom-Probe Field-Ion Microscope (APFIM) facility which will enable microscopic determinations of composition and atomic structure to be made on a wide range of materials in both the surface and the bulk regions of a sample. The atom probe facility will consist of three instruments: (1) A rapid turnover lowtemperature field-ion microscope (built earlier) for initial specimen surveys and microstructure characterization; (2) A general-purpose atom probe (GPAP) for initial microcomposition surveys; and (3) A hish-resolution energy-corrected atom probe (ECAP) for accurate microcompositional analyses, especially for very small (< $10 \mathrm{~nm}$ ) precipitates, alloy phases, etc.

The general purpose atom probe (GPAP), completed last year, has been applied to the determination of the composition and the micro-structure of superalloys. With the imaging and variable-aperture capability of the 
GPAP, selected ares of the alloy specimens, that is single-phase regions, have been isolated for analysis. This is important to alloy designers since the composition of microprecipitates strongly influences the properties of alloys. The GPAP is especially suited for this type of research into structures and composition for precipitates, etc. whose major dimensions are less than about $50 \mathrm{~nm}$. The GPAP is thus extending our measurement capabilities to an important area of materials characterization.

An evaluation of the instrumental factors influencing the compositional analysis of alloys has been initiated and is more than half completed. This work will lead to an optimization of procedures to improve the confidence limits in determinations of composition with our GPAP.

Considerable progress has been made in the construction of the energy-corrected atom probe (ECAP). The main chamber, the straight-path detector, the energy-compensation lens, and the end-flight ion detector have been assembled. The vacuum manifold, gas-supply systems and electronic control units are being constructed.

During the past year, a nickel-base superalloy, RSR 143 (Ni,Al,Mo,Ta), has been analzyed with the GPAP and an analytical transmission electron microscope (TEM). The compositions of the three phases of this alloy, namely the $\sigma$ matrix, the $\sigma^{\prime}$ cuboids and the $\mathrm{DO}_{22}$ platelets were determined, and it was found that the platelets ( 1 ine-scale precipitates) were rich in aluminum and tantalum. The conclusion is that both elements provide for the stabilization of the precipitates in this alloy.

The alloy RSR 224A (Ni,Al, Mo,W) has been studied in cooperation with the Pratt and Whitney Company. It was found that $W$ is present in excess in the small precipitates and indeed is absent from the surrounding matrix. The inference is that further addition of $W$ should provide additional precipitate growth, so that particular mechanical properties may be enhanced.

In conjunction with the comparisons of analyses made using the GPAP and TEM, calculations have been made of the effect of bremsstrahlunginduced Pluorescence on $x$-ray micro-analysis in the TEM. This fluorescence should not be overlooked in the analysis of thin-foil specimens composed primarily of heavy elements.

Measurements have been made on $\mathrm{Ni}_{3} \mathrm{Al}$ to assess factors influencing the analysis of composition with the GPAP. As found by others, increasing the ratio of pulse voltage to dc bias voltage (from 10 percent to 40 percent) results in increases of the measured $\mathrm{Ni} / \mathrm{Al}$ ratio towards the nominal value. Further analysis of these experiments is in progress.

During the next year we will initiate micro-diffusion measurements for metal/metal and metal/semiconductor systems. Ne plan the completion of construction and testing of the ECAP and its application to grain- 
boundary segregation studies in nickel-base superalloys and to interdiffusion measurements of metal layers on semiconductors. We will also continue studies establishing and extending the confidence limits of atom-probe analysis of complex systems.

7. Surface Theory

(S. M. Girvin, P. K. Lam, T. J. Jach, D. E. Ramaker)

Theoretical research is conducted in areas judged userul for the experimental program and to develop a conceptual base for surface and interface properties, processes, interactions, and measurements.

A principal area of investigation has been the quantum Hall effect. This phenomenon occurs in a two-dimensional electron gas (inversion layer) found at an atomically smooth interface between two semiconducting materials. At very high magnetic fields (1OT) and low temperatures (1K), the Hall resistance of such devices is found to take on universal quantized values $\mathrm{h} / \mathrm{e}^{2} \mathrm{i}$ where $\mathrm{h}$ is Planck's constant and $\mathrm{e}$ is the elementary charge. The quantum number $i$ can be an integer or a simple rational fraction.

The main problem of current interest is to develop an understanding of the origin of the fractional quantum numbers. These are believed to arise from a collective many-body ground state due to Coulomb interactions. A new formalism has been developed for doing quantum mechanics in high magnetic fields in two dimensions. The work centers on the use of coherent states which allow formulation by semi-classical wave packet dynamics. This method redices the complexity of computer calculations which so far have limited the number of interacting particles for which the Schroedinger equation can be solved. We are presently in the process of applying this formalism to the fractional quantum number problem using both analytical and numerical Monte Carlo methods.

This year we have predicted the critical value of magnetic field for the liquid-solid transition (Nizner crystallization) of the inversion layer. For this work, a new variational wave function was developed to describe the solid phase. This wave function includes correlations and is thus an improvement over previous Hartree-Fock results. Numerical evaluation of the ground state energy shows that above a critical magnetic fleld corresponding to 6.5 flux quanta per electron, the inversion layer solidifies (see Figure $1 T$ ) and the fractional quantum hall effect will no longer be observed. This prediction is in good agreement with the limiced experimental data presently available and has stimulated considerable new interest in attempts to directly observe wigner crystallization in inversion layers.

Work also continlied on the elucidation of the mechanisms that can give rise to electron- and photon-stimulated desorption of ions from adsorbates on surfaces as well as from single crystals. Particular attention was given to the competitive core-hole decay processes (i.e. resonant photoemission and Auger decay) either before or after escape of the excited electron. The adsorption systems studied included $\mathrm{OH}$ and $\mathrm{H}_{2} \mathrm{O}$ 


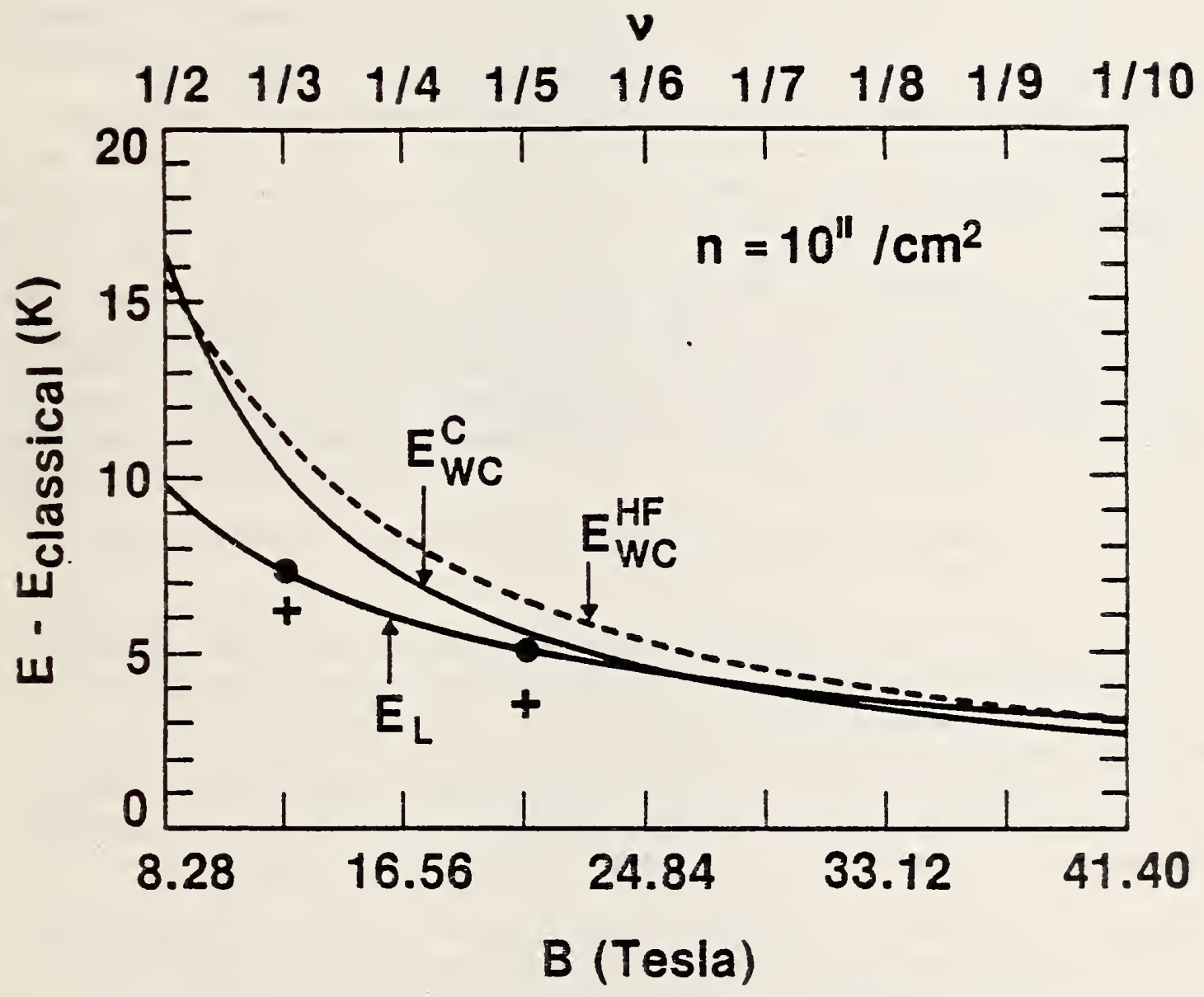

Fig. 11 Ground state energy vs. magnetic field B at an electron density

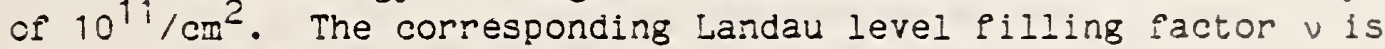
shown on the upper horizontal axis. The dashed line is the Hartree-Fock energy for the solid. The solid line labelled $E_{\text {. }}^{C}$ is the improved solid energy incorporating correlations. $E_{\text {p }}$ is the liguid state energy. Note that for $v^{-1}>6.5$, the solid is more stable than the liquid. 
on $\mathrm{Ti}(001), \mathrm{Cr}(110)$, and $\mathrm{Cu}(100)$ and $\mathrm{O}$ on $\mathrm{Cr}(110)$. A study has also begun to explain the relative $\mathrm{O}^{+}$desorption yields from single-crystal and ion-sputtered $\mathrm{TiO}_{2}$ surfaces. All of these systems have been studied experimentally at NBS (Section 4.B.4). The role of excitonic or defect-like states in the localization of either the initial excited electrons or the two final-state holes, and hence in the desorption process, will be further studied.

Next year we plan to complete the analytic and numerical Monte Carlo descriptions of the fractional quantum number ground state and see the application to inversion layer systems of the semi-classical wave-packet techniques pioneered by Keller for chemical physics problems. Work will continue (in collaboration with Prof. M. W. Cole of the Pennsylvania State University) to develop a scattering theory formalism to describe the repulsive part of the rare-gas metal-substrate interaction.

C. Surface Science Experiments at the Brookhaven

National Synchrotron Light Source

A major undertaking since FY 1981 has been the initiation of an NBS-Naval Research Laboratory collaboration to establish four beam Iines at the Brookhaven National Synchrotron Light Source (NSLS). The Surface Science Division is directly involved in experiments on two of the four beam lines on the $x$-ray ring. These experiments have been delayed due to machine difficulties at Brookhaven.

1. Photon-Stimulated Desorption and Photoemission Spectroscopy (T. E. Madey, R. Stockbauer)

This work is an extension of the experiments performed at the NBS SURF-II facility (see section 4.B.4) to the higher photon energies and more intense light available at the NSLS. Light from a unique, extended-range monochromator $(5-5000 \mathrm{eV})$ will be used to study surface structure and surface bonding of adsorbed molecules. Experimental probes will include photoemission spectroscopy, surface extended $x-r a y$ absorption fine structure, and, particularly, photon-stimulated desorption.

The main experimental ultrahigh vacuum chamber has been designed and constructed, and the sample preparation chamber has been designed and acquired. Ancillary electronics and uhv apparatus (manipulator, LEED/ESDIAD optics, argon ion gun, etc.) have been purchased. The first efforts will be to characterize the roles of both core-level and vaience excitations in the photon-stimulated desorption (PSD) of ions from covalently-bonded surface molecules.

2. Dynamical Effects Associated with Core-Level Ionization (T. J. Jach)

The NBS Quantum Metrology Group under the direction of Dr. R. D. Deslattes is developing an experiment to study electronic effects associated with core-level ionization in atoms, molecules, and simple solids. Specific effects to be studied include dynamicai effects in 
Inner-shell vacancy production, relaxation of the extra-atomic environment, and production of multiple vacancies with or without lonization. One member of the Surface Science Division staff has been assigned to work half-time on this project.

The experiment will consist of a high-resolution crystal monochromator designed to operate between 0.8 and $5 \mathrm{eV}$ in an ultrahigh vacuum. It is planned to study photoemission, Auger-electron emission, and $x$-ray fluorescence from gas and solid targets, particularly in the vicinity of thresholds for core-level ionization. The facility will also be used for extended $\mathrm{x}$-ray absorption $\mathrm{f}$ ine structure and $\mathrm{x}$-ray standing-wave studies of clean surfaces and interfaces.

We have become the first user group to obtain radiation from a port on the NSLS $x$-ray ring. Present work involves the extensive modification and positioning of our bearn output facilities in collaboration with machine physicists at NSLS. We have developed a new generation of photodiodes particularly designed for $\mathrm{x}$-ray synchrotron experiments. These diodes are compatible with ultrahigh vacuum (UHV) systems and are bakeable. We are designing the second-generation UHV experimental chamber for surface-sensitive $x$-ray experiments. This chamber will be particularly suited for $x$-ray standing wave, surface diffraction, and reflectance studies. It will include a manipulator capable of being set to very small angles with high accuracy. 


\section{PUBLICATIONS}

(a) Publications of Past Year

Andreadis, T., Fine, J., and Matthew, J. A. D., "Auger Electron

Emission from the Decay of Collisionally-Excited Atoms Sputtered from Al and Si," Nucl. Instr. and Meth. 209/210, 495 (1983).

Andreadis, T. D., Roush, M. L., Davarya, F., Chambers, G. P., Goktepe, O. F., and Fine, J., "Shape of the Target Region Affected by the Cascade Recoils," Nucl. Instrum., and Meth. 218, 747 (1983).

Bange, K., Grider, D. E., Sass, J. K., and Madey, T. E., "The Surface Chemistry of $\mathrm{H}_{2} \mathrm{O}$ on $\mathrm{Clean}$ and Oxygen-Covered $\mathrm{Cu}(110)$, "Surface Sci. $136,(1984)$.

Benndorf, C. and Madey, T. E., "Adsorption and Orientation of $\mathrm{NH}_{3}$ on Ru(001)," Surface Sci. 135, 164 (1983).

Benndorf, C. and Madey, T. E., "Interaction of $\mathrm{NH}_{3}$ with Adsorbed oxygen and Sodium on Ru(001): Evidence for both Local and Long-Range Interactions," Chem. Phys. Lett. 101, 59 (1983).

Benndorf, C., NObl, C., and Madey, T. E., "H 20 Adsorption on Oxygen-Dosed $\mathrm{Ni}(110)$ : Eormation and Orientation of $\mathrm{OH}(\mathrm{ad}), "$ Surface Sci. 138, 292 (1983).

Bertel, E., Stockbauer, R., and Madey, T. E., "Electron Emission and Ion Desorption Spectroscopy of Clean and Oxidized Ti(0001)," Surface Sci. 141,355 (1984).

Blaszczyszyn, R., Blaszczyszyn, M., Meclewski, R., Melmed, A. J., and Madey, T. E., "Interactions of Sulfur with Nickel Surfaces: Adsorption, Diffusion, and Desorption," Surface Sci. 13i, 433 (1983).

Cage, M. E., Dziuba, R. F., Field, B. F., Williams, E. R., Girvin, S. M., Gossard, A. C., Tsui, D. C., and Hagner, R. J., "Dissipation and Dynamic Nonlinear Behavior in the Quantum HalI Regime," Phys. Rev. Lett. 51, 1374 (1983).

Cage, M. E. and Girvin, S. M., "The Quantum Hall Effect I," Comments on Solid State Physies 11, 1 (1983).

Cage, M. E., Field, B. F., Dziuba, R. F., Girvin, S. M., Gossard, A. C., and Tsui, D. C., "Temperature Dependence of the Quantum Hall

Reistance," Phys. Rev. B. Rapid Commun. 30, 2286 (1984).

Cavanagh, R. R. and King, D. S., "Molecule-Surface Interactions and Dynamics," J. Vac. Sci. Tech. A2, 1036 (1984). 
Cavanagh, R. R., Rush, J. J., Kelley, R. D., and Udovic, T. J., "Adsorption and Decomposition of Hydrocarbons on Platinum Black:

Vibrational Modes from NIS," J. Chem. Phys. 80, 3478 (1984).

Cavanagh, R. R., Rush, J. J., and Kelley, R. D., "Comment on Quantum Motion of Chemisorbed Hydrogen on Ni Surfaces," Phys. Rev. Lett. 52, 2100 (1984).

Cowan, P. L., Hastings, J. B., Jach, T. J., and Kirkland, J. P., "A UHV-Compatible Two-Crystal Monochromator for Synchrotron Radiation," Nucl. Instr. and Method. 208, 349 (1983).

Doering, D. L., Semancik, S., and Madey, T. E., "Coadscrption of Water and Sodium on the Ru(001) Surface," Surface Sci. 133, 49 (1983).

Ederer, D. L., Madden, R. P., Parr, A. C., Saloman, E. B., Rakowsky, G., Cooper, J., Stockbauer; R., Madey, T. E., and Dehmer, J. L., "An Overview of Research at NBS Using Synchrotron Radiation at SURF-II," IEEE Trans. Nucl. Sci. NS30, 1020 (1983).

Egelhoff, Jr., W. F., "Surface Electronic Structure and Screening of 3d-Band Holes in Cu(100)," Phys. Rev. B. B29, 4769 (1984).

Egelnoff, Jr., W. F., "A Core-Level Binding-Energy-Shift Analysis of Adsorption and Desorption," Phys. Rev. B. B29, 3681 (1984).

Egelhoff, Jr., W. F., "A Core-Level Binding-Energy Shift Analysis of $\mathrm{N}_{2}$ on Ni(100)," J. Vac. Sci. Technol. A2, 1013 (1984).

Egelhoff, Jr., W. F., "Surface Electronic Structure Changes Induced by Chemisorption," J. Vac. Sci. Technol. A2, 932 (1984).

Egelhoff, Jr., W. F., "Growth Morphology Determination in the Initial Stages of Epitaxy by XPS," j. Vac. Sci. Technol. A2, 350 (1984).

Egelhoff, Jr., W. $\mathrm{F}_{\text {., }} \mathrm{N}_{2}$ on $\mathrm{Ni}(100)$ : Angular Dependence of the $\mathrm{N}_{1 \mathrm{~s}}$ XPS Peaks," Surface Sci. 141, L324 (1984).

Egelhoff, Jr., W. F., "X-Ray Photoelectron and Auger Electron For'ward Scattering: A New Tool for Studying Epitaxial Growth and Core-Level Binding Energy Shifts," Phys. Rev. B Rapid Comm. 30, 1052 $(1984)$.

Erickson, N. E. and Powell, C. J., "Summary Abstract: Comparison ol $\mathrm{L}_{3}$ Shell-Excitation Energies of 3d, Transition Metals Obtained by AEAPS, and EELS," J. Vac. Sci. Technol. A2, 840 (1984). 
Fine, J., Andreadis, T. D., and Davarya, F., "Measurement of Time-Dependent Sputter-Induced Silver Segregation at the Surface of a Ni-As Ion Beam Mixed Solid," Nucl. Instr. and Meth. 209/210, 521 (1983).

Fine, J., Andreadis, T. D., and Matthew, J.A. D., "Anomalous $\mathrm{N}_{23}$ Aliger Spectra of In and Sn," J. Phys. C. 17, L257 (1984).

Fine, J. and Navinsek, B., "Thin Film Reference Materials for Sputter Depth Profile Calibration," Proceedings of Invited Lectures and Progress Reports, Eleventh Symposium on the Physics of Ionized Gases, edited by G. Pichler, Institute of Physics of the University (Zagreb, Yugoslavia) 1983, p. 223.

Gadzuk, J. W., "Shape Resonances, Overtones, and Electron Energy Loss Spectroscopy of Gas Phase and Physisorbed Diatomic Molecules," J. Chem. Phys. 79, 3982 (1983).

Gadzuk, J. W., "Vibrational Excitation in Molecule-Surface Collisions due to Temporary Negative Molecular Ion Formation," J. Chem. Phys. 79 , 6341 (1983).

Gadzuk, J. W. and Holloway, S., "Trajectory Approach to the Hydrogen Evolution Reaction," Phys. Scripta T4, 86 (1983).

Gadzuk, J. W., "Non-adiabatic Effects in Elementary Surface Reactions: State-to-State Molecular Beam Experiments as a Probe," in Many-body Phenomena at Surfaces, D. Langreth and H. Suhl, eds., Academic Press 517 (1984).

Girvin, S. M., "Hall Effect," Mc-Graw-Hill Encyclopedia of Science and Technology (1984).

Girvin, S. M., "Particle-hole Symmetry in the Anomalous Quantum Hall Effect," Phys. Rev. B. Rapid Commun. 29, 6012 (1984).

Girvin, S. M., "Anomalous Quantum Hall Effect and Two-Dimensional Classical Plasmas: Analytic Approximation for Correlation Functions and Ground State Energies," Phys. Rev. B 30, 558 (1984).

Girvin, S. M. and Cage, M., "The Quantum Hall Effect II," Comments on Solid State Physics 11, 47 (1983).

Girvin, S. M. and Jach, T., "Interacting Electrons in Two-Dimensional Landau Levels: Results for Small Clusters," Phys. Rev. B. 28, 4506 (1983).

Girvin, S. M. and Jach, T., "Formalism for the Quantum Hall Effect: Hilbert Space of Analytic Functions," Phys. Rev. B. 29, 5617 1984). 
Hardy, S. and Fine, J., "Surface Segregation in Liquid Ga-Sn Alloys by AES," Surface Sci. 134, 184 (1983).

Jach, T. and Cowan, P. L., "PIN Diodes as Detectors in the Energy Region $500 \mathrm{eV}-10 \mathrm{eV}, "$ Nucl. Instr. and Methods 208, 423 (1983).

Jach, T. and Powell, C. J., "X-Ray Photoemission Spectroscopy of Environmental Particles," Environmental Sci. \& Technol. 18, 58 $(1984)$.

Jonson, M. and Girvin, S. M., "The Thermoelectric Effect in a Weakly Disordered Inversion Layer Subject to a High Magnetic Field," Phys. Rev. B. 29, 1939 (1984).

Kelley, R. D. and Semancik, S., "On the Mechanism of Fischer Tropsch Synthesis on a Single Crystal Nickel Catalyst," J. Catal. 84, 248 (1983).

Kelley, R. D., Cavanagh, R. R., and Rush, J. J., "Coadsorption and Reaction of $\mathrm{H}_{2}$ and $\mathrm{CO}$ on Raney Nickel: Neutron Vibrational Spectroscopy," J. Catal. 83, 464 (1983).

Klaua, M. and Madey, T. E., "The Adsorption of $\mathrm{H}_{2} \mathrm{O}$ on Clean and Oxygen-Dosed Silver Single Crystal Surfaces," Surface Sci. 136, 42 $(1984)$.

Lam, P. K. and Girvin, S. M., "Liquid-Solid Transition and the Fractional Quantum Hall Effect," Phys. Rev. B. Rapid Commun. 30, 473 (1984).

Landman, U., Kleinman, G. G., Cleveland, C. L., Kuster, E., Barnett, R. N. and Gadzuk, J. W., "Hindered and Modulated Rotations of Adsorbed Diatomic Molecules: States and Spectra," Phys. Rev. B. 29, 4313 (1984).

Madey, T. E., "Early Applications of Vacuum, From Aristotle to Langmuir," J. Vac. Sci. Tech. A2, 110 (1984).

Madey, T. E., Doering, D. L., Bertel, E., and Stockbauer, R., "Electron and Photon Stimulated Desorption: Benefits and Pitfalls," Ultramicroscopy 11,187 (1983).

Madey, T. E., and Stockbauer, R., "The Use of Angle Resolved Electron and Photon Stimulated Desorption for the Determination of Molecular Structure at Surfaces," Rev. Brasil. Apl. Vac. (J. of Brazilian Vacuum Society) 3 , 47 (1983). 
Madey, T. E., Benndorf, C., Doering, D. L., and Semancik, S., "Influence of Surface Additives, Sodium and Oxygen, on the Structure and Bonding of $\mathrm{H}_{2} \mathrm{O}$ and $\mathrm{NH}_{3}$ on $\mathrm{Ru}(001)$, "Proc. of 1984 Internat. Catal. Cong., Berlin (DECHEMÂ, Frankfurt, 1984), Vol. IV, p. 51 .

Melmed, A. J. and Carroll, J. J., "An Approach to Realism in Field Ion Microscopy via Zone Electropolishing," J. Vac. Sci. Tech. A 2, 1388 (1984).

Miskovic, Z., Vukanic, J., and Madey, T. E., "Influence of the Image Interaction of Ion Desorption Processes," Surface Sci. 141, 285

Navinsek, B., Panjan, P., Zabkar, A., and Fine, J., "Determination of Sputtering Yields from a New Procedure for Depth Profiling of Multilayered Structures," Nucl. Instr. and Meth. B2, 670 (1984).

Netzer, F., Doering, D. L., and Madey, T. E., "Evidence for Molecular Reorientation on a Surface: Coadsorption of $\mathrm{CO}$ and $\mathrm{Na}$ on $\mathrm{Ru}(001)$," Surface Sci. 143, 363 (1984).

Powell, C. J., "Inelastic Scattering of Electrons in Solids," in Electron Beam Interactions with Solids for Microscopy, Microanalysis, and Microlithography, D. F. Kyser, H. Niedrig, D. E. Newbury and R. Shimizu, eds. (SEM Inc., Chicago, 1984), pp. 19-31.

Ramaker, D. E., "Comparison of Photon Stimulated Dissociation of Gas Phase, Solid, and Chemisorbed Water," J. Chem. Phys. 80, 183 (1983).

Rousin, M. L., Davarya, F., Chambers, G. P., Andreadis, T. D., He, J., Goktepe, O. F., and Eine, J., "Distribution of Origins of Sputtered Particles and the Shape of the Target Region Affected by the Cascade Recoils," Nucl. Instr. and Meth. B2, 693 (1984).

Rush, J. J., Cavanagh, R. R., and Kelley, R. D., "Neutron Scattering from Adsorbates on Platinum Black," J. Vac. Sci. Technol. Al, 1245 (1983).

Simons, D. G., Brown, M. D., Fine, J., Andreadis, T. D., and Navinsek, B.., "Rutherford Backscattering Analysis of Multilayered $\mathrm{Cr}-\mathrm{Ni}$ Structures to be used for Sputtering Standards," Nucl. Instr. and Meth. 218, 585 (1983).

Stockbauer, R. and Madey, T. E., "Desorption of Ions from Surfaces: Mechanisms of Photon Stimulated Desorption," Annals of the Israel Phys. Soc. 1 , 483 (1983).

Stockbauer, R., Hanson, D. M., Elodstrom, S. A., Bertel, E., and Madey, T. E., "Photon Stimulated Desorption of Ions from Water and Methanol Adsorbed on a Ti(001) Surface," Physica Scripta T4, 126 (1983).

Stockbauer, R., "Instrumentation for Photon Stimulated Desorption," Nucl. Instrum. Meth. 222, 284 (1984). 
Stockbauer, R., Ramaker, D. E., Bertel, E., Kurtz, R. L., and Madey, T. E., "Mechanisms for Photon Stimulated Desorption of $\mathrm{O}^{+}$from Cr(110)," J. Vac. Sci. Tech. A2, 1053 (1984).

Twig8, M. E., "The Effect of Continuum Fluorescence on X-Ray Microanalysis in the Analytical Transmission Electron Microscope," J. Microscopy 134. RP1-RP2 (1984). 
Bange, K., Madey, T. E., and Sass, J. K., "Characterization of OH(ad) Formation by Reaction Between $\mathrm{H}_{2} \mathrm{O}$ and $\mathrm{O}(\mathrm{ad})$ on $\mathrm{Ag}(110)$," Proceedings of ECOSS-6, Surface Sci. (in press).

Bertel, E., Kurtz, R. L., Stockbauer, R., Ramaker, D. E., and Madey, T. E. "Photon Stimulated Desorption of $\mathrm{H}^{+}$Ions From $\mathrm{OH}$ on $\mathrm{Ti}, \mathrm{Cr}, \mathrm{Cu}$, and From Bulk Solid $\mathrm{H}_{2} \mathrm{O}$," Phys. Rev. Rapid Commun., (submitted).

Bertel, E., Stockbauer, R., Kurtz, R. L., Madey, T. E., and Ramaker, D. E., "The Decay Channels of the 3p Resonance in the $3 d$ Transition Metals and Their Relevance to the Mechanisms of Electron- and Photon Stimulated Desorption," Proceedings of ECOSS-6, Surface Sci. (in press).

Bertel, E., Stockbauer, R., Kurtz, R. L., Ramaker, D. E., and Madey, T. E., "Resonant Photoenission and the Mechanism of Photon Stimulated Ion Desorption in Transition Metal Oxides," Phys. Rev. Lett. (submitted).

Butler, J. J., Holland, D. M. P., Parr, A. C., Stockbauer, R., and Buff, R., "Automation of the NBS Threshold Photoelectron-Photoion Coincidence Mass Spectrometer," J. Phys. E. (submitted).

Butler, J. J., Holland, D. M. P., Parr, A. C., and Stockbauer, R., "A Threshold Photoelectron-Photoion Coincidence Spectrometric study of Dimethylether $\left(\mathrm{CH}_{3} \mathrm{OCH}_{3}\right)$," Int'1. J. Mass Spectrom. Ion Phys. (in press).

Cavanagh, R. R. and King, D. S., "Laser Studies of Surface Chemical Reactions," Chemistry and Physics of Solid Surfaces V, Springer Verlag, New York, Inc. (in press).

Ceyer, S. T. and Yates, J. T. Jr., "Ammonia Adsorption on the Ag(311) Surface," Surface Sc1. (submitted).

Ceyer, S. T., Melmed, A. J., Carroll, J. J., and Graham, W. R., "On the Atomic Structure and Optical Constants of (001) Tantalum," Surface Sci. (in press).

Ciszewski, A. and Melmed, A. J., "Epitaxial Growth and Some Properties of Samarium Crystals on Tungsten," $J$. de Physique (submitted).

Ciszewski, A. and Melmed, A. J., "Epitaxial Crystal Growth of HCP Metals on BCC Metals: Dysprosium on Tungsten," J. Cryst. Growth (in press).

Ciszewski, A. and Melmed, A. J., "Epitaxial Crystal Growth of Gadolinium on Iungsten," Surface Sci. Lett. (in press).

Ciszewski, A. and Melmed, A. J., "Surface Self-Diffusion or Dysprosium and Gadolinium," Surface Sci. Lett. (in press). 
Egelholf, Jr., W. F., "Core Level Binding-Energy Shifts in Solids at Surfaces," Surface Sci. Reports (submitted).

Egelhofe, Jr., W. F., "Angle Resolved XPS of the Epitaxial Growth of Cu on $N i(100), "$ Proceedings of the 1st Int. Conf. on the Structure of Surpaces. (submitted).

Egelhoff, Jr., W. F. and Armstrong, R. A., "The Role of Forward Scattering in Angle Resolved XPS," Surface Sci. (submitted).

Fine, J. and Hardy, S., "Methods for Producing Clean Liquid Surfaces," Treatise on Clean Surface Technology, ed. K. L. Mittal, (submitted).

Gadzuk, J. W. and A. C. Luntz, "On Vibrational Lineshapes of Adsorbed Molecules," Surface Sci. (in press).

Gadzuk, J. W. and Norskov, J. K., "Vibrational Excitation, Harpooning, and Sticking in Molecule-Surface Collisions," J. Chem. Phys. (in press).

Heilweil, E. J., Casassa, M. P., Cavanagh, R. R., and Stephenson, J.C., Picosecond Laser Studies of Energy Transfer in Molecules on Surfaces," J. Chem. Phys. (in press).

Heinonen, 0., Taylor, P. L., and Girvin, S. M., "Electron-Phonon Interactions and Breakdown of the Dissipationless Quantum Hall Effect," Phys. Rev. B. (in press).

Holloway, S. and Gadzuk, J. W., "Energy Redistribution and Dissociative Adsorption in Molecule-Surface Collisions Involving Charge Transfer/Surface Hopping," Surface Sci. (in press).

Kelley, R. D., Cavanagh, R. R., Rush, J. J., and Madey, T. E., "Neutron Spectroscopic Studies of the Adsorption and Decomposition of $\mathrm{C}_{2} \mathrm{H}_{2}$ and $\mathrm{C}_{2} \mathrm{H}_{4}$ on Raney Nickel," J. Catal. (submitted).

King, D. S. and Cavanagh, R. R., " Einal-State Resolved Studies of Molecule Surface Interactions," in New Laser and Optical Investigations of Chemistry and structures at Interfaces. Verlag Chemie, (in press).

Madey, T. E. and Benndorf, C., "Influence of Surface Additives (Na and 0) on the Adsorption and structure of $\mathrm{NH}_{3}$ on $\mathrm{Ni}(110), "$ Proc. of ECOSS-6 (Surface Science, in press).

Madey, T. E., Ramaker, D. E., and Stockbauer, R., "Characterization of Surfaces Using Electron and Photon Stimulated Desorption," Ann. Rev. Phys. Chem. (in press). 
Melmed, A. J., Maurice, V., Frank, O., and Block, J. H., "Rare-Earth Crystal Growth from the Vapor: Eu/Re and Eu/W," J. de Physique (in press).

Miskovic, Z., Vukanic, J., and Madey, T. E., "Influence of the Image Interaction on Electron Stimulated Desorption Ion Angular Distribution (ESDIAD)," Proc. of Symposium on Physics of Ionized Gases, SPIG (Dubrovnik, Yugoslavia, August 1984), (in press).

Powell, C. J., "Inelastic Mean Free Paths and Attenuation Lengths of Low-Energy Electrons in Solids," Scanning Electron Microscopy/1984 (in press).

Powell, C. J., "Innershell Ionization Cross Sections, $"$ in Electron Impact Ionization, T. D. Märk and G. H. Dunn, eds. Springer-Verlag (in press).

Shinn, N. D. and Madey, T. E., "Unusual C-O Bond Weakening on a Clean Metal Surface: CO on $\mathrm{Cr}(110), "$ Phys. Rev. Lett. (submitted).

Twigg, M. E., Melmed, A. J., Klein, R., Kaufman, M. J., and Fraser, H. L., "A Microanalytical Study of Secondary Precipitation in a Nickel-Base Superalloy Using Atom Probe Field Ion Microscopy and Analytical Transmission Electron Microscopy," Journal de Physique (in press).

Twigg, M. E., Melmed, A. J., Klein, R., Kaufman, M. J., and Frase, H. L., "A Microanalytical Study of Secondary Precipitation in a Nickel-Base Superalloy With Use of Atom Probe Field Ion Microscopy and Analytical Transmission Electron Microscopy," Proceedings of Workshop on Electron Microscopy (submitted).

Twigg, M. E., Melmed, A. J., Klein, R., Kaufman, M. J., and Fraser, H. L., "A Microanalytical Study of Secondary Precipitation in RSR 143 using Atom Probe Field Ion Microscopy and Analytical Transmission Electron Microscopy," Proc. of Fifth Internat. Symp. on Superalloys (in press).

Udovic, T. J., Kelley, R. D., and Madey, T. E., "The Methanation Activity of $N(110)$," Surface Sci. Lett. (submitted). 
(c) Recent Publications of New Staff Members (Kurtz, Lam, Shinn, Twigg, Udovic, and Wax) Describing Work in Previous Positions

Chou, M. Y., Lam, P. K., and Cohen, M. L., "Calculation of Compton Profile of Beryllium," Phys. Rev. B. 28, 1626 (1983).

Chou, M. Y., Lam, P. K., and Cohen, M. L., "Ab initio Study of Structural and Electronic Properties of Beryllium," Phys. Rev. B. 28, 4179 (1983).

Henrich, V. E. and Kurtz, R. L., "The Electronic Structure of Oxide Surfaces and Surface Defects," Proc. Ixth Int. Vacuum Congress and vth Int. Cong. on Solid Surfaces, Madrid, Spain, 100 (1983).

Henrich, V. E., Kurtz, R. L., and Sadeghi, H. R., "Summary Abstract: Chemisorption on Transition-Metal Oxides and Oxide Supported Noble Metals," J. Vac. Sci. Technol. A1, 1074 (1983).

Janowicz, A. H., Periana, R. A., Buchanan, J. M., Kovac, C. A., Stryker, J. M., Wax, M. J., and Bergman, R. G., "Oxidative Addition of Soluble Iridium and Rhodium Complexes to Carbon Hydrogen Bonds in Methane and Higher Alkanes," Pure Appl. Chem. 56, 13 (1984).

Kurtz, R. L. and Henrich, V. E., "Geometric Structure of the $\mathrm{Fe}_{2} \mathrm{O}_{3}(001)$ Surface: A LEED and XPS Study," Surface Sci. 129, 345 (1983).

Kurtz; R. L. and Henrich, V. E., "Summary Abstract: The Influence of $\mathrm{O}_{2}$ and $\mathrm{H}_{2} \mathrm{O}$ on the Surface Electronic Structure of Corundum Transition-Metal Oxides," J. Vac. Sci. Technol. A2, 842 (1984).

Lam, P. K. and Cohen, M. L., "Calculation of High-Pressure Phases of A1," Phys. Rev. B 26, 5986 (1983).

Lam, P. K. and Cohen, M. L., "Correlation of Superconductivity and Material Properties, " Phys. Lett. 97A, 114 (1983).

Lam, P. K., Chou, M. Y., and Cohen, M. L., "Temperature and Pressure Induced Phase Transition in Be, " J. Phys. C. 17, 1984 (in press).

Lam, P. K. and Chen, M. L., "Dependence of Lattice Constants and BulK Moduli on Pseudopotential Properties," J. Phys. C. (in press).

MCDonald, G. W., Udovic, T. J., Dumesic, J. A., and Langer, S. H., "Equilibria Associated with Cupric ChIoride Leaching of Chalcopyrite Concentrate, "Hydrometal. (in press).

McFeely, F. R., Morar, J. F., Shinn, N. D., Landgren, G., and Himpsei, F. J., "Synchrotron Photoemission Investigation of the Initial Stages of FIuorine Attack on Si Surfaces: Relative Abundance of Eluorosily Species," Phys. Rev. B. 30, 764 (1984). 
Morar, J.F., McFeely, F. R., Shinn, N. D., Landgren, G., and Himpsel, F. J., "Synchrotron Photoemission Investigation: Fluorine on Silicon Surfaces," Appl. Phys. Lett. 45, 174 (1984).

Shinn, N. D., Morar, J.F., and McFeely, F. R., "Spectroscopic Characterization of Fluorinated Silicon Single Crystal Surfaces," J. Vac. Sci. \& Technol. (in press).

Wax, M. J., Stryker, J. M., Buchanan, J. M., Kovac, C. A., and Bergman, R: G., "Reversible $\mathrm{C}-\mathrm{H}$ Insertion/Reductive Elimination in ( $n^{5}$-Pentamethylcyclopentadienyl) ( $r$ imethyl phosphine) iridium Complexes. Use in Determining Relative Metal-Carbon Bond Energies and Thermally Activating Methane," J. Am. Chem. Soc. 106, 1121 (1984). 


\title{
CHEMICAL KINETICS DIVISION
}

\author{
S. E. Stein, Acting Chief \\ SUMMARY OF ACTIVITIES
}

Fiscal Year 1984

1. INTRODUCTION

A. Objectives

The objective of chemical kinetics is to understand the critical lactors controlling chemical change and reactivity. It seeks to both interpret chemical change in terms of individual molecular events as well as develop quantitative theoretical models for these events. The importance and vitality of this area are documented in the "Pimentel Committee Report" to the National Academy of Sciences. In this report, "Chemical Reactivity" was chosen as one of the three "Priority Areas" in chemistry where the greatest opportunities for major advances lie (see, Chemical and Engineering News, Jan. 4, 1984, p. 8).

Exciting experimental breakthroughs and theoretical developments have been made over the last few years that have dramatically expanded the breadth and depth of the discipline. Experimentally, largely as a result of advances in laser and computer technology, a new array of instrumentation has been developed with improved precision and sensitivity which are now yielding increasingly varied and detailed kinetic information. Spurred on by practical concerns such as combustion, these new instruments, alongside more established techniques, have brought certain areas of kinetics to a high degree of development where practitioners are on the verge of devising reliable theories with broad predictive power. The development of these theoretical tools is of critical importance to the field, since they provide frameworks for interpreting as well as generalizing results of individual experiments.

While the number and diversity of reactions investigated by kineticists are increasing at a rapid pace, the area has actually become more cohesive as interrelations between formerly divergent areas become increasingly clear. For instance, unimolecular reaction rate theories developed for neutral species are being widely used to explain critical features of gas-phase ion-molecule chemistry. In a similar manner, results of gas-phase ion chemistry are beginning to provide a solid conceptual foundation for solution ion chemistry, a poorly understood area of immense practical impcrtance. 
In addition to its role as a major fundamental area of science, chemical kinetics has long been an area directly associated with the solution of practical problems. The chemistry of combustion, radiation and the atmosphere are examples of areas whose development depends directly on advances in chemical kinetics.

Over the past several years the scope of the Chemical Kinetics Division has expanded significantly. Just three years ago its primary emphasis was on gas-phase reactions of neutral species. Through recent additions of staff and changes in research direction, it is now roughly equally divided into groups studying ion and neutral reactions and on studies in the gas and condensed phases. In addition, the data centers (ion and neutral) are broadening their efforts in kinetic data evaluation.

Since predictive theories are required by both experimentalists and "data evaluators", the advancement of these theories provides a key unifying factor for the diverse activities in the division. In fact, it is expected that the Division may ultimately be able to offer not only NBS evaluated data but also NBS "calibrated" predictive models for data not currently available.

Central to the broadening of the Division's scope have been two competency development programs. One, entitled "Transformations of Complex Organic Compounds", has enabled us to add personnel and equipment in order to apply the exact principles of small molecule kinetics to reactions of more complex substances. The second program, "Physical and Chemical Effects of Ionizing Radiation on Matter" has enabled the growth of existing programs on gas-phase ion-molecule chemistry to include more complex reactions in both the gas phase and solution.

\section{B. Goals and Activities}

The scientific objective of the Chemical Kinetics Division is to understand the molecular factors governing chemical change and reactivity. This involves both the measurement of fundamental kinetic properties in well-defined chemical systems and the development of theoretical concepts and predictive methods. The Division is particularly interested in areas which either lead to broad improvements in theory or assist in the solution of important national problems.

In pursuit of the above objective, our work involves the following types of research: (1) developing new experimental systems for expanding the scope of kinetic measurement; (2) measuring rates of reactions needed for interpreting more complex processes; (3) developing theory and determining rate constants needed for theoretical advancement, (4) compiling, evaluating and disseminating chemical kinetics data, (5) interpreting complex chemical reactions in terms of single-step molecular events. 
The Chemical Kinetics Division devotes a part of its efforts to serving.as a technical resource for other bureau or other agency programs. We consider this activity to be an integral part of our mission, and a key means of applying our scientific expertise to industrial, defense, and environmental problems. Moreover, these projects are directly related to, and strongly supportive of, the basic long-range scientific objectives of the division. In FY 84 we participated in the following NBS and OA programs :

Office of Standard Reference Data

- The Chemical Kinetic Data Centers (ion and neutral) for data compilation and evaluation.

Department of Energy

- Compilation and evaluation of combustion chemistry data

- Chemistry of hazardous waste incineration

- Pulse radiolysis studies of electron transfer in solution

- High pressure mass spectrometric studies of ion kinetics and thermodynamics.

Department of Defense

- Laser-induced chemistry

- Chemical theory of carbon

- Kinetics of the decomposition of nitroaromatics

- Multiphoton ionization processes

- Tandem mass spectrometric studies

- Ion-cyclotron resonance studies of carbon cluster ions

National Aeronautics and Space Administration

- Elementary kinetic studies of stratospheric reactions

- Chemical kinetics of planetary atmospheres

- Chemical fundamentals of soot formation

- Evaluation of stratospheric chemical kinetic data 


\section{CHEMICAL KINETICS AND ION KINETICS ENERGETICS DATA CENTERS}

J. T. Herron, R. F. Hampson, Jr., W. Tsang, F. Westley, G. W. Zumwalt R. J. Cretanovic, S. G. Lias and R. D. Levin

\section{A. Introduction}

The Chemical Kinetics Data Center and the Ion Kinetics and Energetics Data Center are responsible for providing compilations and evaluations of numerical data on the kinetics of chemical reactions, and the thermochemical properties of ionic and free radical species. They provide this information to scientists in other disciplines, such as the analytical and biological sciences, and to modelers analyzing very complex chemical systems such as encountered in comoustion and atmospheric chemistry. Although the major source of information contained in the existing data bases is derived from experimental studies, our goal is the development and application of theoretical methods for both the analysis and extrapolation of existing experimental data, and the prediction of the behavior of systems for which there is no data. Over the past year the Data Centers have been physically relocated to make them contiguous. This will allow the two centers to more easily share facilities and equipment as well as to encourage joint planning and programs.

B. Chemical Kinetics Data Center

(J. T. Herron, R. F. Hampson, Jr., W. Tsang, F. Westley

G. W. Zumwalt and R. J. Cvetanovic)

The goals of the Chemical Kinetics Data Center are to provide compilations, bibliographies and evaluated chemical kinetics data bases for neutral species in support of science and technology, to develop new or improved theoretical models for the prediction of chemical kinetic data, and to develop cost effective methods for the acquisition, evaluation, and dissemination of chemical kinetic data to the scientific and technical community. This center has served for many years as a national resource in supplying evaluated chemical kinetic data, data compilations, and annotated bibliographies covering a broad range of scientifically and technologically important systems. We have been involved in the chemistry of stratospheric ozone depletion since it was first recognized as a serious problem, and continue to assist in providing the authoritative chemical kinetic data base used in modeling stratospheric chemistry. We provide data compilations and evaluations which address critical problems in combustion chemistry and allow a more thorough testing of kinetic theory.

One of our major goals in the area of data evaluation is to provide a definitive set of evaluated rate data for use in combustion modeling. This activity is supported by the office of Basic Energy Sciences of DoE. The first major output of that program, "Chemical Kinetic Data Base for Methane Combustion", is complete and has been submitted to WERB for publication in JPCRD. This compilation contains evaluated data on over 250 chemical reactions. In this evaluation, a common theoretical framework, transition state theory, was used to fit data measured over a wide 
range of temperature and pressure. An example of the results of this application of theory to combustion reactions can be seen in Fig. 1, where data on the reaction $\mathrm{C}_{2} \mathrm{H}_{6} \geqslant 2 \mathrm{CH}_{3}$ are shown. Remarkably, the theoretical Pramework shows that the majority of the data in the older literature are valid, contrary to earlier conclusions, and that one can derive rate expressions for this reaction over the entire range of temperature and pressure encountered in a practical combustion systems. This powerful approach to data evaluation has been widely used in this new data evaluation, and will be used in extensions of this data base.

The extension of this work to include the reactions of propane, propyl radicals, methanol, and the $\mathrm{CH}_{2} \mathrm{OH}$ radical with all species considered in the methane system is in progress. Over ninety reactions are involved.

In order to increase the pace of our data evaluation efforts and focus efforts in the area we have organized an informal, voluntary committee of chemical kineticists and modelers to provide external inputs for this work. The present members of our committee are:

David Gutman
Norman Cohen
David Colden
Joe Michael
James Miller
Charles Westbrook
Bruce Klemm
Thomas Dunning
Robert Hampson
Wing Tsang

David Gutman Norman Cohen David colden Joe Michael James Miller Charles Westbrook

Thomas Dunning Wing Tsang

\author{
Illinois Institute of Technology \\ Aerospace Corporation \\ SRI International \\ Brookhaven National Laboratory \\ Sandia Corporation \\ Lawrence Livermore Laboratory \\ Brockhaven National Laboratory \\ Argonne National Laboratory \\ NBS, Chemical Kinetics Division \\ NBS, Chemical Kinetics Division
}

A related project on developing a compilation of experimentally determined rate constants for combustion reactions continues. Papers for the period 1977-1981 have been abstracted and all data entered into the HP-1000 computer system. This compilation is being extended to include all data published through 1982. This bibliographic activity is the prelude for the critical evaluation of combustion data for systems more complex than methane.

An evaluation of stratospheric reactions of halogen cortaining species was completed as part of the Data Center contribution to the NASA Panel for Data Evaluation and has been published by the jet Propulsion Laboratory. These data were also reviewed and evaluated for the CODATA Task Group on Chemical Kinetics. The complete report of that task group has now been prepared and edited by the Data Center and submitted to jPCRD for publication.

The development of a data base management system continues under a contract to R. J. Cvetanovic. Most work on the identification and nomenclature of inorganic and organic compounds is complete. Work on the 

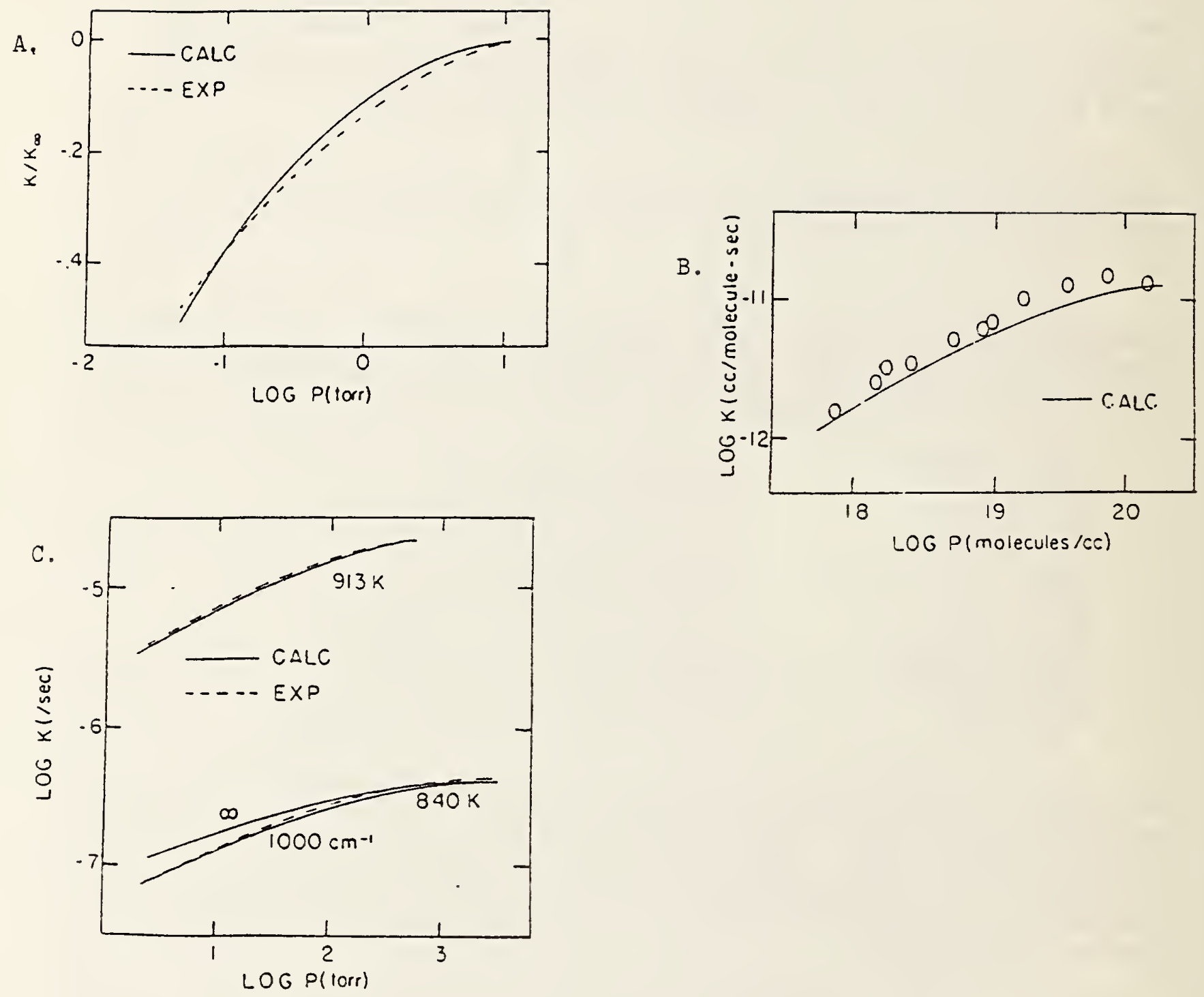

Figure 1 Comparison between experiments and calculations for the reaction $\mathrm{C}_{2} \mathrm{H}_{6} \rightleftarrows 2 \mathrm{CH}_{3}$

A. $2 \mathrm{CH}_{3} \rightarrow \mathrm{C}_{2} \mathrm{H}_{6} \quad 370 \mathrm{~K}$ (strong collider)

B. $2 \mathrm{CH}_{3} \rightarrow \mathrm{C}_{2} \mathrm{H}_{6} \quad 1400 \mathrm{~K}$ (argon, $300 \mathrm{~cm}^{-1}$ step size)

C. $\mathrm{C}_{2} \mathrm{H}_{6} \rightarrow 2 \mathrm{CH}_{3} \quad 840 \mathrm{~K}$ and $913 \mathrm{~K}\left(1000 \mathrm{~cm}^{-1}\right.$ and strong collider $)$ 
actual searching routines for chemical reactions is underway and should be largely complete this year. Each component of the system is being tested on a suitably desizned data base.

\section{Ion Kinetics and Energetics Data Center}

(S. G. Lias and R. D. Levin)

The goals of the Data Center are to collect and maintain an upto-date file of experimental data pertaining to the thermochemistry of lons (ionization potentials, appearance potentials, and equilibrium constants) and the kinetics of ion-molecule reactions, to make compilations of these data and to publish evaluations of the collected data for use by the scientific and technological communities.

The Data Center is closely associated with the Ion Kinetics Group which, as described in Section 4., can supply benchmark measurements of ion thermochemical quantities to aid in data evaluation activities.

(1) Major publications this year were:

"Evaluated Gas Phase Basicities and Proton Affinities of Molecules; Heats of Formation of Protonated Molecules" by Sharon G. Lias, Joel F. Liebman, and Rhoda D. Levin has been accepted for publication in the Journal of Chemical and Physical Reference Data, where it will appear in the third issue of 1984.

This publication includes:

(a) A comprehensive review of the field of gas phase proton affinity measurements.

(b) A table containing evaluated gas basicities and proton affinities, as well as the original literature data from which these were derived, for 784 atomic and molecular species.

(c) A tabie containing evaluated heats of formation of all the molecular species and of the protonated molecules.

(d) An annotated bibliography of references on gas phase proton affinity determinations.

(e) A bibliography of references to heats of formation of the relevant neutral molecules.

"Ion Thermochemistry" by S. G. Lias, J. E. Bartmess, J. F. Liecman, J. L. Holmes, and R. D. Lev in is a comprehensive and complete compilation of evaiuated heats of formation of more than 3000 positive and negative ions, now being prepared for publication in the Journal of Physical and Chemical Reference Data: 
Preliminary work on the evaluation of ion heats of formation is completed, and a computer printout of a first draft of the tables for the manuscript is in hand. Figure 2 shows an example page from the publication. An exhaustive and comprehensive review, proof-reading and editing effort, coupled with a cross-checking of recent literature, is now under way in the Data Center. Outside collaborators J. F. Liebman (University of Maryland, Baltimore County), J. E. Bartmess (University of Tennessee), and J. L. Holmes (University of Ottawa) are also reviewing the tables.

(2) Archive maintenance included literature searching, identification of articles containing data, copying, abstracting, and encoding data into the computer. Approximately 250 articles containing approximately 2000 measurements were processed this year.

(3) Data center automation included the implementation of a researchable data base for the archival data on ionization potential and appearance potential data, in collaboration with Dr. David Neumann of the Chemical Thermodynamics Data Center and the completion of programs permitting the formatting of encoded evaluated data into forms suitable for publication.

\section{Future Plans}

As part of the implementation of our long range plan, there will be major changes in the management and operation of the Chemical Kinetics Data Center in FY 1985. John Herron has become the Director, and will spend full time on the design and implementation of Data Center programs. The broadening of the scope of the Chemical Kinetics Data Center continues to be a priority objective, as is the increased involvement of other data evaiuators, from inside and outside NBS. To help foster this development, the Data Center will be heavily involved in the planning and organization of the International Conference on Chemical Kinetics, to be held at NBS in June, 1985. Other changes in the direction and operation of the data center will be put in place as plans are developed over the coming year.

A major objective in the coming year will be the implementation of the database management system and the conversion of existing data bases into forms suitable for inclusion in the new system. Progress in developing software for the new system has reached the point at which search routines for chemical reactions will be tested and modifications made based on an analysis of the test runs. We will continue to work closely in this endeavor with the Ion Energetics and the Chemical Thermodynamics Data Centers. We will also be considering various options concerning the identification, abstraction, and entry of data into the new system. 


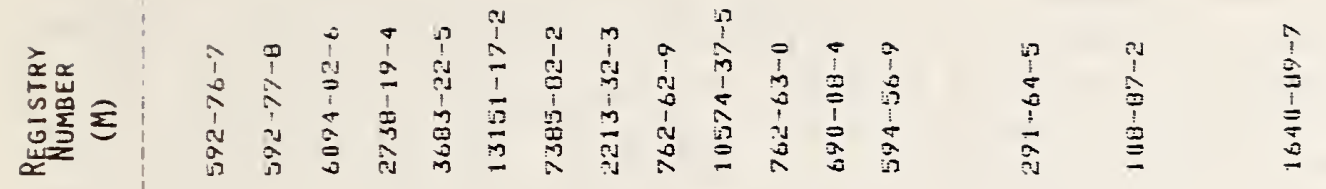

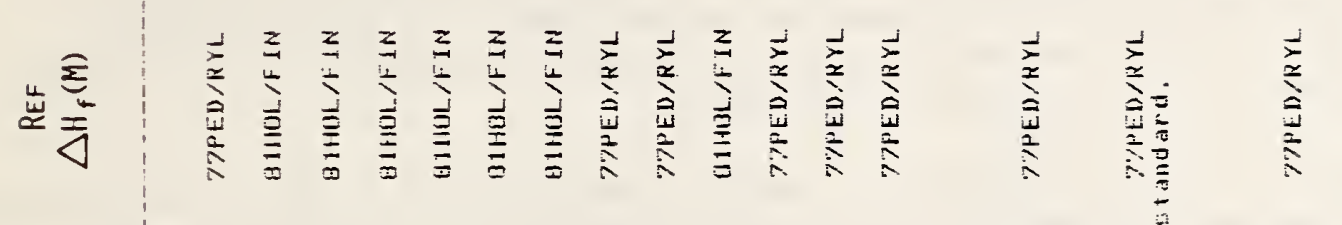

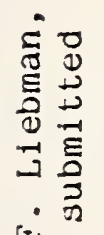

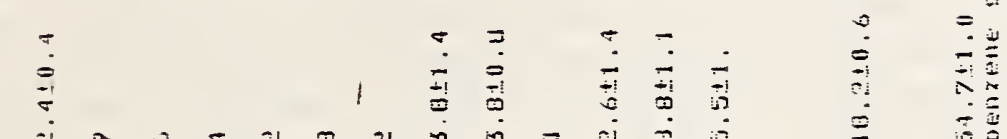

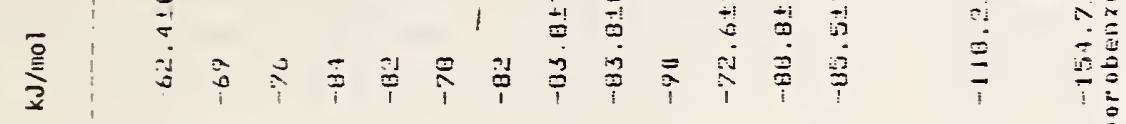

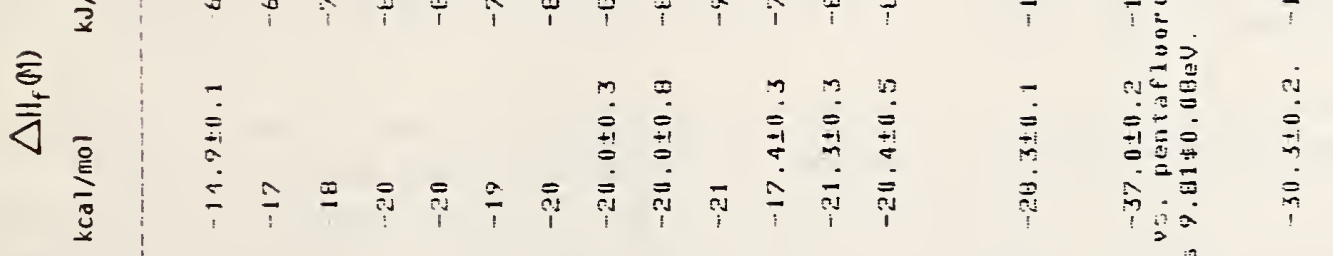

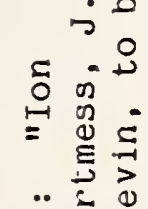

造茎

$\ddot{\pi} \dot{0} \dot{0}$

$\underset{0}{0} \dot{0} \dot{2}$

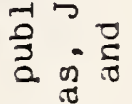

-

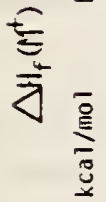

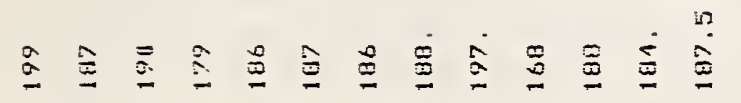

尚

$\stackrel{\infty}{\infty}=\infty$

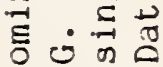

舟

잉

ह

के का

$\infty$ 芩

웡 $\frac{0}{2}$

谣送

产焉う。

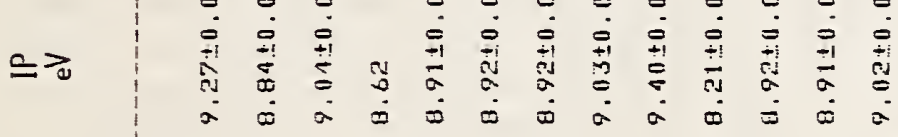

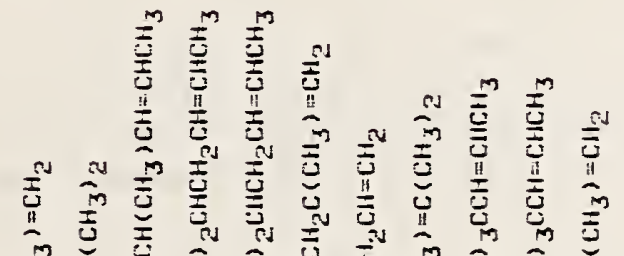

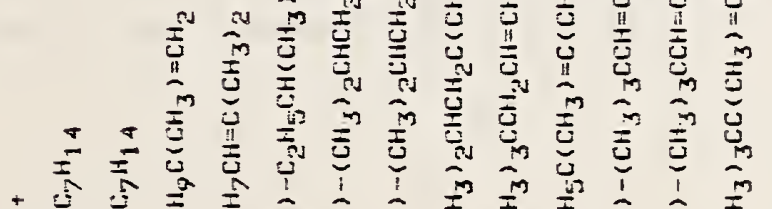<smiles>C1CCCCC1</smiles><smiles>CC1CCCCC1</smiles><smiles>OC1CCCC1</smiles>

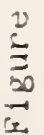

考 
Evaluation activities in the coming year will center around the continuing development of the combustion chemistry data base. The data base will be expanded to include additional reactions of three and four carbon compounds including propene, the butanes and butenes, and iso-propyl and butyl radicals. The number of reactions of these new species with species already in the data base is about 192 . The total data base will then consist of over 500 evaluated chemical reactions. Since relatively little information is available for larger species, extension to larger molecules will increasingly require the development and application of theoretical methods.

Work will also continue at a much reduced scale on the maintenance of the stratospheric chemistry data base, particularly with that part concerned with halogen containing molecules. In connection with this activity, Bob Hampson will continue to serve a member of the NASA Panel for Data Evaluation and the CODATA Task Group on Chemical Kinetics. This work is supported by NASA.

A new project on the evaluation of chemical kinetic data related to the industrially important problem of $\mathrm{SF}_{6}$ dielectric breakdown was initiated in FY 1984 by John Herron and will be continued as a Data Center activity this year. This is part of a much broader area of the applied chemistry of plasma deposition and plasma etching as related to electronics manufacturing and the coating industry.

On-going work on the publication of evaluated heats of formation of ions will be completed in the Ion Kinetics Data Center. The routine literature-searching, abstracting, and data encoding activities of the Data Center will continue in order to maintain up-to-date archives of ionization potential, appearance potential, and proton apfinity data.

Formulation of plans for an evaluated compilation of data on ion-molecule rate constants and reaction cross sections, and the initiation of a literature search for that project will begin in the Ion Kinetics Data Center. This work will complement previous compilations in the field, which are now seriously out of date. Data from these earlier publications will be combined with that from the literature of the past five years, and will be organized for inclusion in a computerized data base.

In addition, a data base to aid in the standardization of literature reference formats, and the design of a small auxiliary data base to aid in retrieval of papers from the library will be implemented for the joint use of this Data Center and the Chemical Kinetics, Chemical Thermodynamics and Electrolyte Data Centers. 
Dr. J.E. Bartmess of the University of Tennessee has compiled and evaluated the data on thermochemistry of negative ions for the forthcoming publication on heats of formation of ions. He has expressed an interest in permanently maintaining computersearchable up-to-date archives of anion thermochemistry as an activity of our data center. Such an arrangement will be formalized. 


\section{KINETICS OF NEUTRAL SPECIES}

R. Billmers, W. Braun, R. L. Brown, A. Fahr, L. L. Griffith, J. W. Hudgens, M. J. Kurylo, A. Laufer, M. Manka, R. I. Martinez,

D. Robaugh, M. D. Scheer, S. E. Stein, L. E. Szegvary, W. Tsang and J. A. Walker

\section{A. Introduction}

There are two main parts to this program. The first deals with the kinetics of the reactions of atoms and small polyatomic molecules. It is a traditional area of research at NBS. The second is our competence program entitled "Transformation of Complex Organic Compounds". In this area we are attempting to develop an understanding of the behavior of large organic molecules under severe conditions at the single-step elementary reaction level.

Reactions of small molecules and atoms are of interest for their key role in numerous gas phase chemical processes as well as for their use in the development of chemical kinetic theory. The ability to unambiguously detect trace concentrations of small reactive intermediates by optical techniques is largely responsible for the high degree of development of this area. Included in this component of our program are the development and application of lasers for studies of high temperature kinetics (B4), studies of small oxygen containing free radicals (B5) rate measurements of reactions involving unsaturated free radicals ( 36 ) and oxidation chemistry (37).

The competence development part of our program is devoted to studies of larger, more complex species under severe reaction conditions. These studies represent an expansion of the scope of research in our Division, in which the exact methods of smali molecule kinetics are applied to reactions of more complex molecules. Specifically, studies are intended to lead ultimately to the capability of prediction and thus permit detailed understanding of the whole range of problems where the stability and reactivity of large organic molecules are of key importance. This program has now reached its full planned scope. Shock tube, very low pressure pyrolysis and liquid phase pyrolysis systems are all fully operative and producing data. We are currently concentrating on aromatic systems. These range from systems where phenyl, phenoxy and benzyl radicals are of key importance, to the pyrolysis of anthracene and the reactions of large resonance stabilized radicals in solution and, at the iarge molecule iimit, theoretical studies on graphite. In all cases our fundamental approach has yielded significant new insights. These will be discussed in detail in $\mathrm{B} 1-\mathrm{B} 2$ and $\mathrm{C}$. The MS/MS system has finally been delivered and appropriate reactors have been constructed (Section B3a). Dr. J. W. Hudgens has joined us from the Naval Research Laboratory. He is bringing into the division expertise in multiphoton ionization of transient reaction intermediates (Section $\mathrm{B} 3 \mathrm{~b}$ ). This powerful new technique will lead to a determination of structures and reacivivities of intermediates not detectable by other means and is expected to play an important role in our program. 
1. Elementary Kinetic Studies of Aromatic Species

(A. Fahr, D. Robaugh, S. E. Stein and W. Tsang)

This is an important aspect of our competence program. Under severe conditions aromatic type compounds are of key importance. of concern here are the stability and reactivity of benzyl type radicals and the phenyl radical. There is at present considerable uncertainty regarding their properties. We have used two techniques, the heated single pulse shock tube and the very low pressure pyrolysis reactor to study such systems. Our results during the current year have demonstrated the validity of our experimental procedures. We have discovered a new mechanism for nitrobenzene decomposition, obtained a new value for the heat of formation of phenyl radical, demonstrated that literature reports of a new mechanism for the decompostion of alkybenzene are in error and provided the first data on radical combination reactions involving pheny 1 radicals.

The heated single pulse shock tube has been used to study the mechanism and rates of decomposition of a number of aromatic compounds. These include phenyl iodide, nitrobenzene, ethylbenzene and isopropylbenzene. Complementing these studies, the decomposition of ethylbenzene and isopropylbenzene has been studied using the very low pressure pyrolysis (VLPP) technique.

The work on phenyl iodide was aimed at establishing the validity of our procedures for studying reactions involving phenyl radical production. We have found cyclopentane to be a satisfactory radical trap. A Iimit has been found ( .1 percent) above which all phenyl radicals wili be converted to benzene through abstraction from cyclopentane. The rate expression for phenyl lodide decomposition has been found to be $k(\phi I \rightarrow \phi \cdot+I \cdot)=10^{-15.0}$ exp $(-33200 / \mathrm{T}) / \mathrm{sec}$. The rate constants are within 50 percent of earlier measurements in a flow system. However, the rate expression differs considerably. Assumption of a temperature independent recombination rate leads to $\Delta \mathrm{H}_{\mathrm{f}}(\phi \cdot)=341 \mathrm{~kJ} / \mathrm{mol}(298 \mathrm{~K})$. This is about $13 \mathrm{~kJ}$ higher than the number recommended in a recent review. On the basis of detailed. balance the new thermochemistry leads to a recombination rate of 1010 $\ell / m o l-s e c$. This is an order of magnitude slower than comparable reactions of resonance stabilized radicals. If the lower heat of formation is used a recombination rate of $2 \times 10^{9} \mathrm{l} / \mathrm{mol} \cdot \mathrm{sec}$ is gredicted.

For nitrobenzene decomposition, the main process involves the splitting of the weak $\mathrm{C}-\mathrm{N}$ bond. However from mass balance considerations and the detection of phenol and its relative concentration under a wide variety of concentrations we find that there is an important minor channel (30 percent) involving isomerization to nitrate followed by cleavage of the O-NO bond. The rate expression for $\mathrm{C}-\mathrm{N}$ bond fission is found to be

$$
k\left(\phi-\mathrm{NO}_{2} \rightarrow \phi \cdot+\mathrm{NO}_{2}\right)=10^{15.4} \exp (-33000 / \mathrm{T}) / \mathrm{sec}
$$


Combination of this rate and the thermochemistry leads to a reverse rate of $2.5 \times 10^{9} \mathrm{l} / \mathrm{mol}-\mathrm{sec}(1100 \mathrm{~K})$ and a $1 / \mathrm{T}^{3 / 2}$ dependence. Here, as above, we are obtaining combination rates that are close to an order of magnitude slower than that from alkyl and resonance stabilized radicais. The important question is thus whether this is characteristic of reactions involving unsaturated radicals.

These studies of nitrobenzene permitted us to assess the capabilities of the heated shock tube. Nitrobenzene has a boiling point of $210^{\circ} \mathrm{C}$. It also is known to be extremely "sticky". After some initial problems we found no obstacles in preparing and holding mixtures in the sub-hundred ppm levels. We have also been able to make quantitative determinations of compounds such as diphenyl, diphenylether and phenol in the sub ppm level. Thus it would appear that heating the shock tube opens up a vast number of low volatility organic compounds to study. The problems that we have encountered deal with the storage and transport of these substances at $150-250^{\circ} \mathrm{C}$ range. Under these conditions surface enhanced pyrolytic reactions can occur. We feel that these represent soluble problems and are exercising much greater care in these matters.

The ethylbenzene and isopropylbenzene studies originate from the remarkable claims by Troe's group in Gottingen that ethylbenzene pyrolysis proceeds $\mathrm{via} \mathrm{C}-\mathrm{H}$ bond cleavage. This unexpected result, if true, would considerably alter current ideas of hydrocarbon pyrolysis mechanisms. We employed both heated shock tube and VLPP techniques to test these claims.

In our shock tube studies of ethylbenzene pyrolysis, in the presence of propyne as a radical trap, we found the main reaction product to be toluene. Clearly, benzyl radical must be the precursor. In the absence of the radical trap we find large quantities of styrene. In the inhibited decomposition of isopropylbenzene styrene is also the main reaction product. This demonstrates that the 1 -phenylethyl radical is a precursor to styrene. The implication of this study is that the 1 -phenylethyl radical and styrene detected in real time by Troe and coworkers are products of a radical chain decomposition and are not products of an initial decomposition processes. In our VLPP studies of alkylbenzene decomposition we pyrolyzed ethylbenzene and isopropylbenzene with the objective of making definitive mass balance measurements. In the case of ethylbenzene, methyl and benzyl radicals were the sole detectable products and, using di-tert-butyl peroxide as a $\mathrm{CH}_{3}$ radical source, $>85$ percent of the decomposed ethylbenzene generated methyl radicals. In the case of isopropylbenzene decomposition, methyl radicals and styrene were the oniy products observed. These studies showed that $>85$ percent of the 1 -phenylethyl radicals formed by $\mathrm{C}-\mathrm{C}$ bond breaking rapidly lost $\mathrm{H}$-atoms and were detected as styrene. This demonstrates that if, as proposed by Troe, the 1-phenylethyl radical were formed in ethylbenzene pyrolysis by benzyl $\mathrm{C}-\mathrm{H}$ bond breaking, it would have been efficiently detected in our experiments as styrene. Since no styrene was observed in our ethylbenzene pyrolysis experiments, benzylic $\mathrm{C}-\mathrm{H}$ bond breaking is not competitive with $\mathrm{C}-\mathrm{C}$ bond breaking. 
Together, VLPP and shock tube studies convincingly demonstrate that no revision to conventional pyrolysis mechanisms are needed for alkylbenzenes.

We now plan to use our heated shock tube to study the reactions of phenyl and $\mathrm{OH}$ radicals with a number of unsaturated systems. These are reactions that involve two possible reaction channels, dispiacement and abstraction. We believe that our comparative technique can yield high accuracy absolute rates and branching ratios. A systematic study of such systems should yield information of great scientific and practical interest. For abstraction processes, it will lead to detailed comparisons with saturated systems. Displacement can be considered a combination of addition and radical decomposition. Can the data be quantitatively rationalized in such a context? Finally, we intend to continue with thermal decomposition studies. In particular, we are interested in thermal sources of $\mathrm{OH}$ and phenyl radical (in addition to that discussed above). We will also carry out additional studies on nitroaromatic systems.

VLPP studies of phenyl radicals with unsaturated molecules, specifically, acetylene and ethylene, are planned for the next year. Phenyl radical recombination will serve as a competitive channel against which to compare these measurements. Carbon deposition by phenyl radicals will also be investigated. This is an entirely unexplored area which is expected to interface with our theoretical studies of graphite molecules (see Section C3).

\section{Thermal Stabililty of Alkyl Radicals}

(W. Tsang)

In earlier work we reported on our conclusions regarding the necessity for higher heats of formation for all the simple alkyl radicals (except for $\mathrm{CH}_{3}{ }^{\circ}$ ). A paper has been completed and submitted for publication. Figure 3 iilustrates the magnitude of the differences in terms of the equilibrium heats of formation of the radicals as deduced from our analysis of existing data for alkane and alkyl radical decomposition and the reverse processes and the currently used values. These results are of key importance as we enlarge our data base to include an increasingly larger range of compounds, since a minimal requirement must be that the thermodynamics be satisfied. Our analyses lead to the following best rate expressions for alkyl radical decomposition.

$$
\begin{aligned}
& \mathrm{k}\left(\mathrm{nC}_{3} \mathrm{H}_{7} \rightarrow \mathrm{C}_{2} \mathrm{H}_{4}+\mathrm{CH}_{3} \cdot\right)=10^{13.1} \exp (-15259 / \mathrm{T}) / \mathrm{sec} \\
& \mathrm{k}\left(\mathrm{iC}_{3} \mathrm{H}_{7} \cdot \rightarrow \mathrm{C}_{3} \mathrm{H}_{5}+\mathrm{C}_{4} \cdot\right)=10^{13.3^{4}} \exp (-18104 / \mathrm{T}) / \mathrm{sec} \\
& \mathrm{k}\left(\mathrm{sC}_{4} \mathrm{H}_{9} \cdot \rightarrow \mathrm{C}_{3} \mathrm{H}_{6}+\mathrm{CH}_{3} \cdot\right)=10^{12.97} \exp (-14886 / \mathrm{T}) / \mathrm{sec} \\
& k\left(\mathrm{sC}_{4} \mathrm{H}_{9} \cdot \rightarrow-\mathrm{C}_{4} \mathrm{H}_{8}+\mathrm{H} \cdot\right)=10^{13.11} \exp (-18498 / \mathrm{T}) / \mathrm{sec} \\
& k\left(\mathrm{sC}_{4 \mathrm{H}_{9}} \cdot \rightarrow \mathrm{C}-\mathrm{C}_{4} \mathrm{H}_{8}-2+\mathrm{H} \cdot\right)=10^{12.62} \exp (-17652 / \mathrm{T}) / \mathrm{sec}
\end{aligned}
$$




$$
\begin{aligned}
& k\left(\mathrm{sC}_{4} \mathrm{H}_{9} \cdot \rightarrow t-\mathrm{C}_{4} \mathrm{H}_{8}-2+\mathrm{H} \cdot\right)=10^{12.66} \exp (-17225 / \mathrm{T}) / \mathrm{sec} \\
& k\left(\mathrm{tC}_{4} \mathrm{H}_{9} \cdot \rightarrow \mathrm{iC}_{4} \mathrm{H}_{8}+\mathrm{H} \cdot\right)=10^{13.92} \exp (-18910 / \mathrm{T}) / \mathrm{sec} \\
& k\left(t \mathrm{C}_{5} \mathrm{H}_{11}+i \mathrm{C}_{4} \mathrm{H}_{8}+\mathrm{CH}_{3}\right)=10^{13.03} \exp (-15011 / \mathrm{T}) / \mathrm{sec}
\end{aligned}
$$

The systematic trends in the these expressions, within the context of thermochemical kinetics, provides the basis for the prediction of the rates of decomposition of all alkyl radicals.

3. New Kinetic Measurement Techniques

(J. W. Hudgens, R. I. Martinez)

Progress in the area of kinetics depends to a large degree on advances in our ability to detect reactive intermediates and products. Part of our Competence Initiative is devoted to developing new detection methods for polyatomic species of concern in our program. In particular, we have begun to apply the methods of multiphoton ionization (MPI) and tandom mass spectrometry (MS/MS) for the analysis of large organic reaction intermediates.

\section{a. Multiphoton Ionization of Free Radicals} (J. W. Hudgens)

The development of multiphoton ionization over the past few years has given kineticists a new method with which to probe the spectroscopy and dynamics of molecules which could not be detected by currently available, laser-induced f?uorescent methods. Since most molecules and free radicals do not fluoresce, MPI greatly expands the number of different chemical species that can be detected with lasers.

Multiphoton ionization works by subjecting molecules to intense visible or ultraviolet laser light that causes them to absorb photons until they ionize. Usually the first step involves a simultaneous absorption of two or more photons which promote the molecule into an excited electronic state. The excited state molecule absorbs an additional photon and ionizes. Since ions are easy to detect, the technique is very sensitive.

The most important reaction systems involve free radicals. At present, the data base for radical detection by MPI is small, but we are developing a base useful for future kinetic studies. Research efforts this year have produced methods for the MPI detection of allyl, 2-methyallyl $\mathrm{CH}_{2} \mathrm{~F}, \mathrm{CF}$, $\mathrm{SiF}, \mathrm{ClO}, \mathrm{BrO}$, and $\mathrm{CH}$ radicals (and their deuterated counterparts). The detection of allyl and 2-methylallyl radicals is very significant because it extends MPI detection to the important class of conjugated pi-bonded radicals.

Allyl radicals appear in many thermal, photochemical, and surface catalytic reactions. Yet no laser methods were available for their optical detection. Allyl and 2-methylallyl radicals were detected in the 

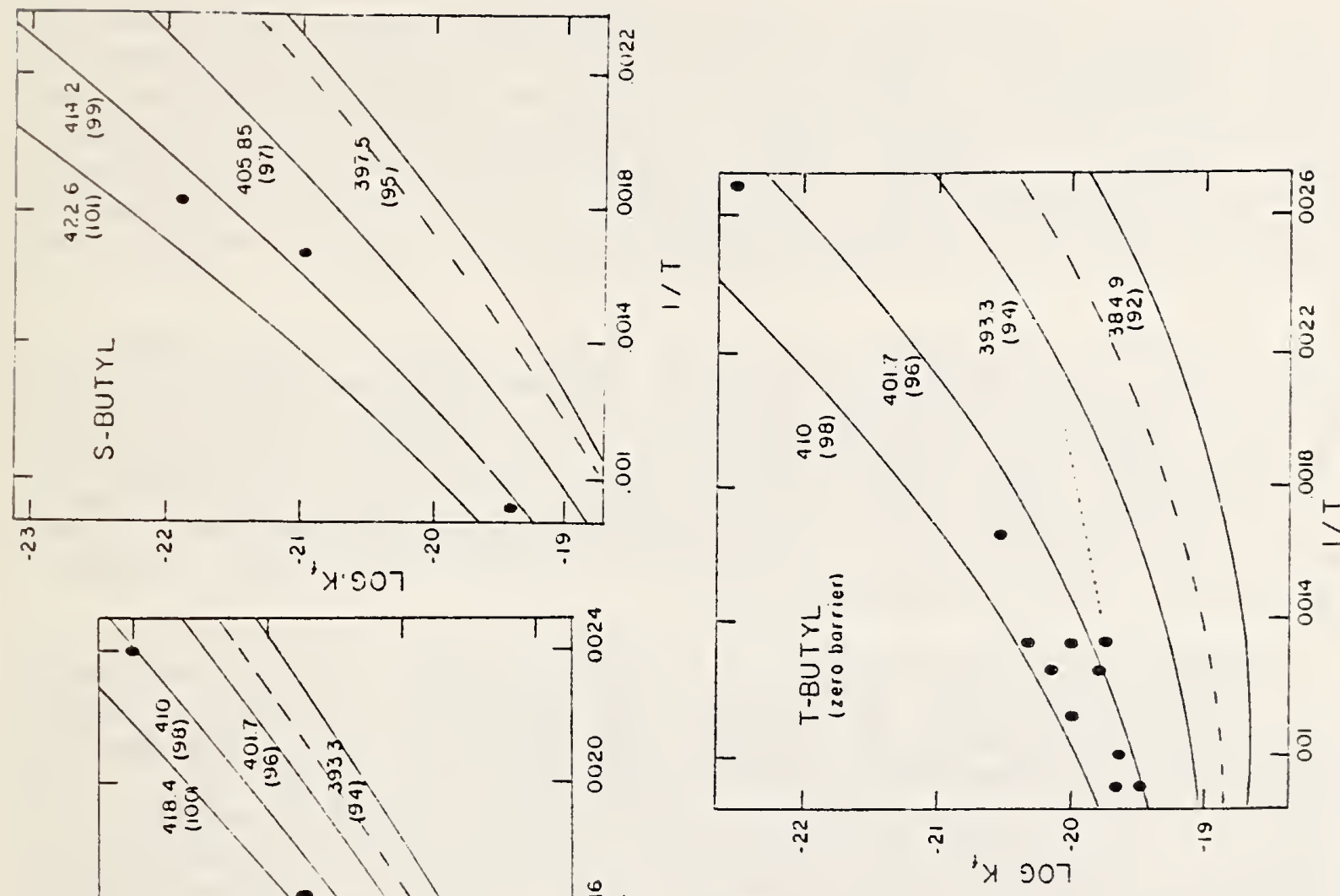

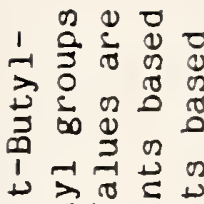

-

$\vec{\imath} \stackrel{0}{*} \stackrel{2}{0}$

苛范

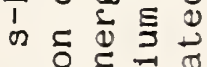

$\rightarrow \rightarrow$

ते $=0$

앙 0 으 =

ᄂ

○ิ

-

$\because$ क क वे

ᄌ리리

융물

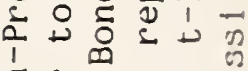

c. क क

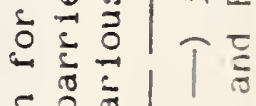

-

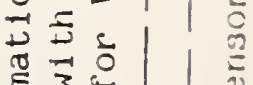

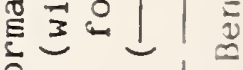

正

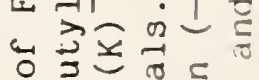

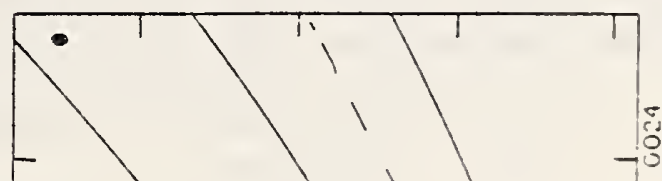

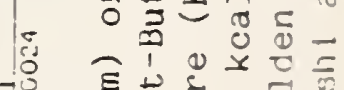

至

क ल के एँ

क के तै

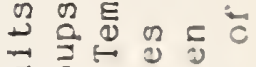

इ 50 $\frac{2}{2}=$

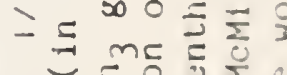

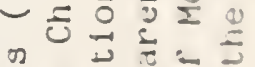

出

ป็

ट

U - क क

Eᄎำ

$5: x=$

$=0 ; 3$

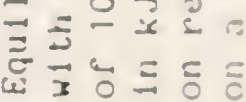

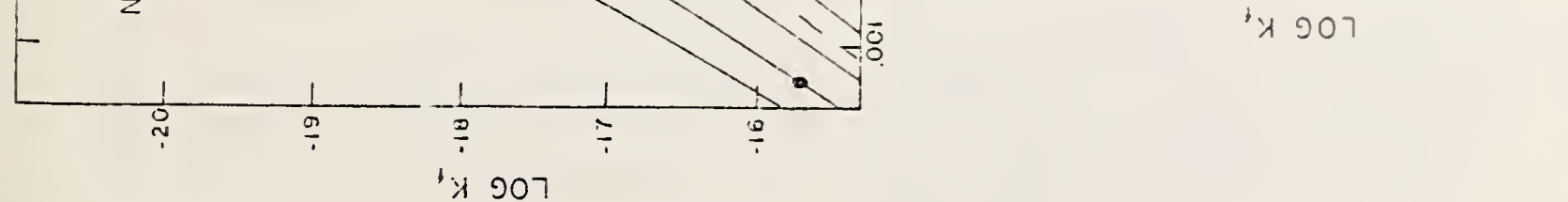

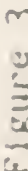


effluent of a flow reactor. The allyl radicals were formed by the reactions of fluorine atoms with cyclopropane and propene. The 2-methylallyl radicals were formed by the reactions of fluorine and chlorine atoms with isobutene. Focused laser light between $480-535 \mathrm{~nm}$ induced two photon absorptions preparing the $3 \mathrm{~s}^{2} \mathrm{~A}$, Rydberg states of the radicals. Absorption of two additional photons ionized the excited Rydberg radicals. The ionization process produced only molecular ions--an important result because it enhances detection sensitivity. Before this work the ns Rydberg states of allyl radicals were unknown.

C10 and Bro radicals are important in reactions involving stratospheric ozone. MPI detection of $\mathrm{C} 10$ and Bro was developed using a tunable dye laser between 417-465 $\mathrm{nm}$. Haiogen atoms were reacted with ozone in the flow reactor to generate the radicals. The MPI spectra were generated by simultaneous three photon absorption which prepared excited Rydberg states of $\mathrm{C} 10$ and Bro. The excited Rydberg radicals then absorbed one or two more photons and ionized. All of the states of Bro detected in this study had not been previously reported.

The work cited above are typical experiments used for the development of the MPI radical detection data base. By generating a catalog of MPI detectable species we can use selective and sensitive laser techniques to probe reactions systems which involve these radicals. Particularly important are the class of aromatic free radicals and their reaction products (which includes allyl radicals). Attempts to detect members of this class of radicals will be nade this year. Laser strategies used to selectively ionize them may include two frequency MPI, production of doubly charged ions, and laser induced charge exchange schemes.

b. MS/MS Tandem Mass Spectrometry (R. I. Martinez)

A new long-range study of the dynamics of neutral-neutral and ion-neutral reaction systems has been initiated. The focus of this sutdy is the development of a real-time analytical methodology which involves the combination of species-specific ionization via 2-color multiphoton ionization and structural analysis via MS/MS tandem mass spectrometry (i.e., 2-color MPI-MS/MS). The intended use of this technique is as a diagnostic tool for monitoring directly in real-time unknown polyatomic reactive transients in complex reacting mixtures.

The technical basis for daveloping 2-color MPI-MS/MS is that the direct, real-time analysis of multicomponent mixtures by mass spectrometry can be divided into two parts:

(1) the ionization of the mixture components and

(2) the quantitation of each component on the basis of its respective ion signal intensity.

For quantitation, one must be able to do either (a) or (b):

(a) find a parent or fragment ion which is unique for a particular mixture component, or

(b) ionize only one component at a time in the presence of all the the other inixture components. 
None of the ionization techniques used routinely (electron impact, photoionization, chemical ionization, etc.) can satisfy requirements (a) or (b) for all components of complex mixtures which can give rise to isomeric and/or isobaric parent and/or fragment ions. The only ionization technique which presently can provide species-specific ionization (i.e., satisiy requirement (b) above) is 2-color MPI (muliphoton ionization wherein one $\lambda_{1}$ is used for up-pumping and a second $\lambda_{2}$ is used for ionization).

While 2-color MPI can provide specific and ultra-sensitive photoionization, it cannot provide unique structural identification of an unknown polyatomic component in a complex multicomponent mixture containing isomers and/or isobars unless a synthetic sample exhibits all the same optical 2-color MPI transitions as the unknown. Clearly, synthetic reference samples are not possible for complex polyatomic radicals. To date, only small, simple radicals have been studied.

In dealing with unknown species, it is necessary to characterize the molecular structure of the ion precursor. That may be accomplished through the use of the complementary technique of MS/MS tandem mass spectrometry. Tandem mass spectromeetry exploits the ion iragmentation patterns which are characteristic of the structure of a compound. Thus the detailed ion-fragmentation mapping obtained in the MS/MS by collision-induced dissociation (CID) of ions formed by "soft" 2-color MPI should allow one to uniquely identify any component in a complex mixture.

The specially designed NBS MS/MS tandem mass spectrometer is now in-house and we have been proceeding with the requisite testing, and modifications needed to accommodate the MPI.

The first phase of the planned research program involves (1) development of 2-color MPI schemes; (2) measurement and analysis of 2-color MPI "breakdown curves"; and (3) measurement and analysis of CID "breakdown curves".

During FY 1985, we will initiate studies on the CID dynamics of selected nolecules. Also, Ne will attempt to develop MPI detection scriemes for the propargyl radical for use in neutral kinetics studies pertinent to the atmosphere of Titan. This work is supported in part by NASA.

4. Laser Studies of High Temperature Chemical Kinetics (W. Braun and M. D. Scheer)

The following three projects describe our use of $\mathrm{CO}_{2}$ lasers ior studying high temperature reactions. The first two projects use a laser as a unique heating source, while the last use the laser to perturb reactions already occurring in high temperature environments. This riork was supported in part by the Air Eorce Office of Scientific Research. 


\section{a. A New High Temperature Reactor.}

During the past year a very rapid, high temperature heating method has been developed. Small volumes of fused silica can be heated to incandescence and beyond through the absorption $10 \mathrm{micron}$ infrared radiation at power density levels in the neighborhood of $1 \mathrm{~kW} / \mathrm{cm}^{2}$. By using the focused beam of a $\mathrm{CW} \mathrm{CO}$ laser ((P2O) line of the 001-100 band) to heat only the orifice end of a fused silica reactor tube, one can achieve very short heating times for gases that flow through the tube and expand adiabatically out of the orifice into an evacuated region.

When chemically reactive gases are allowed to flow at near sonic velocity through a small volume heated by quartz walls irradiated in the above fashion, high temperatures and short reaction times are readily achieved. When the gas is subsequently cooled by adiabatic expansion into a low pressure region, all collision dependent secondary processes are arrested particularly those with significant activation energies. Such a reactor can be used to obtain rate parameters under high temperature, short time conditions not readily attained with other heating methods. By thus extending the range of experimental temperatures at reduced reaction times, additional bond breaking processes can be made to occur and the kinetics of higher activation energy decomposition channels may be isolated and characterized. The multichannel decomposition of cyclobutanone was used to demonstrate the applicability of such a reactor.

It was shown that when a dilute mixture of cyclobutanone in helium flows through the laser heated orifice, it is exposed to the high temperature for only about 20 microseconds. During such short reaction times secondary reactions tend to be reduced and it was demonstrated that the formation of propylene from the secondary isomerization of cyclopropane could be minimized. At orifice temperatures near $1200 \mathrm{~K}$, propylene + CO was shown to be formed directly from the cyclobutanone. The activation energy for this high temperature channel was found to be about 330 $\mathrm{kJ} / \mathrm{mole}$ compared to 242 and 218 for the two lower channels that form cyclopropane + $\mathrm{CO}$ and ethylene + ketene respectively.

b. A New Comparative Method for Determining High Temperature Activation Energies

The new rapid flow, high temperature reactor can be used to precisely determine activation energies by comparing the rate of product formation with the rate of emission of black body photons from the heated orifice at a given wavelength and temperature. Towards this end, a thin film of graphite was deposited on the inside surface of the orifice. The laser was slowly modulated at a frequency of $1 / 2 \mathrm{~Hz}$ with a 50 percent duty cycle. It was found that when a 60:1 mixture of helium and t-butyl iodide flowed through the cyclically heated orifice, the I atoms produced were in phase with the temperature modulation. This temperature modulation was detected by measuririg the spectral radiancy of the quartz orifice at 660 $\mathrm{nm}$ and the I atcms by resonance fluorescence at $162 \mathrm{~nm}$ using an iodine resonance lamp and a solar-blind photomultiplier. For temperatures between 1000 and $1500 \mathrm{~K}$, the black body emission of photons at wavelengtins 
below 600 nn may be described by the Wien approximation. Under these circumstances, the ratio $\left(R_{2} / R_{1}\right)$ of spectral radiancies at two different temperatures, $T_{2}$ and $T_{1}$ obeys the following relation:

$$
\frac{T_{2}-T_{1}}{T_{2} T_{1}}=\frac{k \lambda}{h c} 2 n\left(\frac{R_{2}}{R_{1}}\right)
$$

Since the production of I atoms is governed by the Arrhenius law, the ratio $\left(I_{2} / I_{1}\right)$ for their rates of formation at the same two temperatures is given by the similar relation:

$$
\frac{T_{2}-T_{1}}{T_{2} T_{1}}=\frac{R}{E_{a}} 2 n\left(\frac{I_{2}}{I_{1}}\right)
$$

The activation energy in terms of the measured quantities $\left(R_{2}, R_{1}, I_{2}, I_{1}\right)$ and relative to the energy of the black body photons emitted at $660 \mathrm{~nm}$ is therefore

$$
\Xi_{a}=\frac{\text { hc } \ln \left(\frac{I_{2}}{I_{1}}\right)}{\lambda \ln \left(\frac{R_{2}}{R_{1}}\right)}
$$

where at $660 \mathrm{~nm},(\stackrel{\text { hc }}{\longrightarrow})$ is $43.3 \mathrm{kcal} / \mathrm{mole}$. As an example of the application of this measurement ${ }^{\lambda}$ methodology the t-butyl iodide decomposition jielded an activation energy of about $170 \mathrm{~kJ} / \mathrm{mole}$ for scission of the $\mathrm{C}-\mathrm{I}$ bond in the neighborhood oi $1000 \mathrm{~K}$.

\section{c. Enhancement of Unimolecular Decompositions Nean the} Second Order Limit.

The departure of systems undergoing chemical change from an equilibrium distribution of energies among their molecular degrees of sreedom occurs oniy when rates of reaction are very large. Ignition and diffusion flame phenomena, occurring in heterogeneous and unmixed fuel-oxidant systems, as well as flame propagation through thoroughly mixed zaseous combustibles, are examples of such rapid high temperature chemical processes. Cne important property of these rapidly reacting systems is the existence of a competition between the rate of collisional activation and the rate of surmounting the activation barrier ieading to successful reaction. At low temperatures and high pressures, collisional activation maintains an equilibrium Boltzmann distribution of energy required to overcome the activation barrier so that the process is activation limited. Since the collision rate varies as $T^{1 / 2}$ wille the rate of overcoming the barrier varies as exp( $-\Xi / R T)$, it is readily seen that for any given pressure there exists a temperature beyond which the latter rate vill surpass the former. Under these circumstances the high-energy "talz" of 
the Boltzmann distribution becomes depleted with a consequent lowering of the overall reaction rate. Unimolecular decompositions are the simplest examples exhibiting such fall-off behavior and are characterized by a depletion of those molecules with vibrational energies near and beyond that required to surmount the activation barrier. The pumping of infrared laser energy into the high end of the vibrational energy distribution of the decomposing molecules provides a means of enhancing their rates of decomposition. A quantitative comparison of these rates with and without measured doses of laser radiation will yield relative efficiencies for the transfer of vibrational energy at levels near the activation energy barrier by IR photons compared to heat-bath collisions. Recombination processes on the other hand demonstrate the inverse effect where the reaction exothermicity must be removed by collision with a heat-bath molecule in order to stabilize the adduct. Thus, only collisions which are efficient in removing energy rather than supplying it will enhance the recombination reaction rate and the absorption of IR photons will decrease the recombination rate.

Intense infrared radiation is therefore capable, in principle, of pumping the vibration-rotational manifolds of a reactive molecules and thereby provide a means for decreasing the depletion factor in the Boltzmann distribution at high energies. It has been experimentally demonstrated that pumping the lowest vibrational energy levels of a reactive molecule with an appropriate resonant wavelength can, under favorable circumstances, enhance the rate of reaction. The very high vibrational-rotational energy levels near the top of the activation barrier on the other hand, are very closely spaced in a quasi-continuum so that pumping the high energy part of the Boltzmann distribution should be virtually wavelength independent.

Preliminary evidence for such an effect has been obtained when ethyl iodide diluted twenty fold in 5 torr of helium was passed through a one inm diameter fused silica orifice coated with a thin film of graphite. The orifice was heated by a 15 watt $\mathrm{CO}_{2}$ laser beam (P20 Iine of the 001-100 band at 10.6 micrometers) that was chopped at a frequency of $1 / 2 \mathrm{~Hz}$ with a 50 percent duty cycle. The temperature excursion of the orifice walls during this modulated heating cycle was found radiometrically to be between 1025 and $1275 \mathrm{~K}$, where heating to the temperature maximum occurred during the one second that the laser was on and cooling to the temperature minimum occurred during the one second that the laser was of $f$. It was found that the I atoms produced at a given temperature during the modulation cycle were always greater when the laser was on compared to when it was off. A much smaller effect was observed for $t$-butyl iodide under similar modulation conditions. This observation is consistent with the fact that the ethyl iodide is much further in the fallof than is the t-butyl iodide with twice as many active oscillators. Since alkyl iodides do not have strong ground state absorptions at 10.6 micrometers, it is unlikely that radiative heating of the substrate alkyl iodides can account for these results. Further measurements at different pressures, orifice temperatures, and laser wavelengths are planned in order to determine 
whether the observed enhancement of the rates of these high temperature decompositions can be ascribed to a laser induced repopulation of the high energy end of the depleted vibrational energy distribution.

\section{Kinetics of Free Radical Reactions \\ (M. J. Kurylo)}

The scope of this program is the nigh sensitivity detection and chemical characterization of small gas-phase free radicals (atoms, diatomics, and triatomics). These reactions are important because of their ubiquitous involvement in gas-phase chemistry ranging from acid rain to the depletion of stratospheric ozone, the incineration of hazardous waste to the design of improved combustors, the effects of increased internal energy on kinetics and mechanisms to the elucidation of the chemical composition of planetary atmospheres.

Studies completed the past year, supported by NASA, include measurements (by plash photolysis resonance fluorescence) of the kinetics of acetonitrile $\left(\mathrm{CH}_{3} \mathrm{CN}\right)$ with $\mathrm{Cl}$ atoms and $\mathrm{OH}$ radicals. These studies were aimed at providing input for calculations of $\mathrm{CH}_{3} \mathrm{CN}$ lifetimes in the stratosphere and troposphere. The results, however, when compared with available literature data on $\mathrm{Cl}$ and $\mathrm{OH}$ reactivity with other substituted methanes, provides evidence for decreased reactivity between strong electrophiles. This lowering of the rate constant with increasing electron affinity of the substituents is at cdds with some of the commonly used semi-empirical algorithms for rate constant estimation. As such, our findings represent an important contribution to the needed data base for improved estimation schemes.

Additional studies have focused on the measurement of $\mathrm{HO}_{2}$ reaction rates utilizing flash photolysis kinetic absorption spectroscopy. In particular, cur determinations of the $\mathrm{HO}_{2}$ self reaction rate as a function of $\mathrm{N}_{2}$ and $\mathrm{O}_{2}$ pressure yield a low pressure extrapolation in good agreement with a number of other low pressure studies. In addition, the pressure dependences we observe quantitatively support recent rate constart assessments. Interest in this reaction stems not only from its importance in atmospheric and combustion chemistry but aiso from its consideration as a subject of extensive theoretical treatment regarding both the stabiiity of the $\mathrm{H}_{2} \mathrm{O}_{4}$ dimer reaction intermediate, and the accelerated reactivicy attributed to water vapor complexation.

In the coming year we plan to continue these experiments with emphasis on the $\mathrm{HO}_{2} / \mathrm{NC}_{2}$ combination reaction in order to explain the discrepancies in the reported fall off behavior. In addition, we will study the cross-disproportionation reaction of the $\mathrm{HO}_{2}$ and $\mathrm{CH}_{3} \mathrm{O}_{2}$ radicals. During the past year, we have gained considerabie expertise in monitcring $\mathrm{HO}_{2}$ via absorption spectroscopy. Information regarding our detection sensitivity as a function of wavelength and the sensitivity of our calculated second order rate constants to fluctuations in anaiysis lamp intensity and signal to noise ratios have permitted us to def 1ne the optimum conditions for investigating the cross-disproportionation reaction between $\mathrm{HO}_{2}$ and $\mathrm{CH}_{3} \mathrm{O}_{2}$. This reaction is important not only in the 
atmospheric oxidation of methane and other hydrocarbons but also in low-temperature combustion systems. Furthermore, given the rather good data bases for the $\mathrm{HO}_{2}+\mathrm{HO}_{2}$ and $\mathrm{CH}_{3} \mathrm{O}_{2}+\mathrm{CH}_{3} \mathrm{O}_{2}$ reactions, the cross-system lends itself for interesting theoretical considerations regarding the factors influencing radical radical disproportionation. Experimental measurements will commence in the near future under conditions specified by our recent extensive modeling calculation. Ideally, we would like to have supporting results from monitoring the decay of both radical species. However, due to the large differences in self reaction rates of the two species, precise kinetic measurements will not always be possible for both radicals at each set of initial concentrations. Nevertheless, our calculations indicate that suitable compiementary measurements can be made to fully characterize the kinetics of this reaction.

During the forthcoming year we will collaborate with members of the Center for Analytical Chemistry on the application of diode laser spectroscopy (second harmonic detection) to kinetic systems. While our goal is for an apparatus of general utility in monitoring free radical kinetics or delineating reaction mechanisms through trace product analysis, our initial thrust will be at high sensitivity $\mathrm{HO}_{2}$ detection to complement our kinetic spectroscopic work. Our planned experiments will differ from some very recent work in other Iaboratories by focusing on a different $\mathrm{HO}_{2}$ bond system and on the sensitivity of second harmonic detection. Inherent to such measurements will be the determination of pressure broadening parameters and the effect of water vapor on the infrared line strengths. The effective coupling of the expertise of the collaborating individuals will hopefully pave the way for successful future investigations.

6. Reactions of Biradicals and Unsaturated Eree Radicals (A. Fahr and A. Laufer)

The objective of this program is to produce and characterize biradicals and unsaturated free radicals. The efforts are specifically concerned with reactions of these unique species leading to a quantitative understanding of their chemistry. The experimental approach is to use ilash photolysis to generate free radicals and/or excited species, kinecic spectroscopy to follow species concentrations and gas chromatography for end-product analysis. Isctopic substitutions are applied to clarify mechanistic processes. In these cases, mass spectroscopy of the final products is the analytical method of choice.

Our recent effort3, supported by the NASA Plaretary Atmospheres Program, have been directed towards understanding the mechanism of formation and the chemistry of the vinylidene unsaturated biradical $\left(\mathrm{H}_{2} \mathrm{CC}\right)$. This species, the smallest of a large class of unsaturated biradicals, was initially identified by us on the basis of its characteristic absorption in the vacuum ultraviolet. We have been able to decermine that the particular state of vinylidene under observation is that cf the lowest Iying excited triplet, about $1.5 \mathrm{eV}$ ( $35 \mathrm{kcal} / \mathrm{moi}$ ) above the ground singlet state. Our initial examination of the chemistry of this unique species involved a study of its rate constant for removal, via 
collisional quenching by $\mathrm{He}$, and a determination that the commonly invoked intermediate in $\mathrm{C}_{2} \mathrm{H}_{2}$ systems (often referred to as $\mathrm{C}_{2} \mathrm{H}_{2}{ }^{*}$ ) is chemically equivalent to vinylidene. In a recent paper, which has appeared this year in Icarus, we have applied both BEBO and BSBL techniques to calculation of hydrogenation abstraction rate constants from hydrocarbon molecules by vinylidine which are important in the atmospheres of the outer planets.

In addition, we have been able to identify alternate sources of triplet vinylidene. These results suggest that the species may, in fact, be significantly more important in the photochemistry of hydrocarbons than previously expected. In particular, the spectrum attributable to $\mathrm{H}_{2} \mathrm{C}=\mathrm{C}$ has been observed following the vacuum ultraviolet photolysis of $\mathrm{C}_{2} \mathrm{H}_{4}$. In the ethylene system, the $\mathrm{H}_{2} \mathrm{C}=\mathrm{C}$ is not a primary photofragment but increases rapidly to a maximum and then decays with a rate constant characteristic of its quenching by helium. The buildup of $\mathrm{H}_{2} \mathrm{C}=\mathrm{C}$ with time, in this system, suggests that the previously observed absorption is due, not only to vinylidene, but also it is a vibrationally cold molecule.

A rate constant of the order of $10^{-11} \mathrm{~cm}^{3}$ molecule-1 $\mathrm{s}^{-1}$ which represents the sum of both quenching and reaction (probably addition) of both protonated and deuterated triplet vinylidene with $\mathrm{C}_{2} \mathrm{H}_{4}$ and $\mathrm{C}_{2} \mathrm{D}_{4}$, respectively, has been derived. The work will soon appear in the Journal of ?hotochemistry.

While the direct observation of vinylidene is of great scientific interest, the importance of the radical in hydrocarbon chemistry remains unknown. This year we have determined the extinction coefficient of vinylidene from which we will be able to ascertain the quantum yields for vinylidene formation in the photodissociation of hydrocarbons of planetary importance. The extinction coefficient measurements are based on a study of the photodissociation of vinyl chloride. Though triplet $\mathrm{H}_{2} \mathrm{CC}$ now has been observed from $\mathrm{C}_{2} \mathrm{H}_{2}$ and $\mathrm{C}_{2} \mathrm{H}_{4}$ photolysis and as a product of the reaction of two triplet $\mathrm{CH}_{2}$ radicals, vinyl chloride nas certain advantages. Conceptually, if the photodissociation proceeds through a $i, i$ elimination of $\mathrm{HCl}$, the remaining fragment is vinylidene whose concentration will be equal, to that of the $\mathrm{HCl}$. Since $\mathrm{HCl}$ can be determined absolutely, the absolute concentration of $\mathrm{H}_{2} \mathrm{CC}\left(3_{\mathrm{B}_{2}}\right)$ becomes a tractable quantity. The possible products of tine dissociation of $\mathrm{C}_{2} \mathrm{H}_{3} \mathrm{Cl}$ : $\mathrm{Cl}\left(\mathrm{P}_{2 / 2}, 1 / 2\right), \mathrm{C}_{2} \mathrm{H}_{2}, \mathrm{HCl}$ and $\mathrm{H}_{2} \mathrm{CC}\left({ }_{\mathrm{B}_{2}}\right)$ all have well characterized absorptons in the vacuum ultraviolet. We have obtained time-resolvec spectra of these several species from which we deduce the primary process proceeds by direct absorption to an excited singlet

$$
\mathrm{C}_{2} \mathrm{H}_{3} \mathrm{Cl} \stackrel{\mathrm{hv}}{\longrightarrow} \mathrm{C}_{2} \mathrm{H}_{3} \mathrm{Cl}{ }^{1}\left(\mathrm{n}, \pi^{*}\right)
$$

followed by an intersystem crossing to a high ribrational level of the vinyl chloride triplet $\left(\pi, \pi^{*}\right)$ at $4.0 \mathrm{eV}$ above the ground singlet.

$$
\mathrm{C}_{2} \mathrm{H}_{3} \mathrm{Cl} \quad{ }^{i}\left(\mathrm{n}, \pi^{*}\right) \longrightarrow \mathrm{C}_{2} \mathrm{H}_{3} \mathrm{Cl} \quad 3\left(\pi, \pi^{*}\right)
$$


The unimolecular dissociation of the triplet, $k_{\text {uni }}=4 \times 10^{7} \mathrm{~s}^{-1}$, leads, in a spin-allowed process, to triplet vinylidene $\left(\mathrm{H}_{2} \mathrm{CC}\right)$ and $\mathrm{HCl}\left(\mathrm{X \Sigma}^{+}\right)$

$$
\mathrm{C}_{2} \mathrm{H}_{3} \mathrm{Cl} 3\left(\pi, \pi^{*}\right) \longrightarrow \mathrm{H}_{2} \mathrm{CC}\left({ }^{3} \mathrm{~B}_{2}\right)+\mathrm{HCl}\left(\mathrm{X \Sigma} \Sigma^{+}\right)
$$

The equivalence of $\mathrm{HCl}$ and $\mathrm{H}_{2} \mathrm{CC}$ having been determined allows us to obtain $\varepsilon\left(\mathrm{H}_{2} \mathrm{CC}\right)$, the extinction coefficient, $=420 \mathrm{~atm}^{-1} \mathrm{~cm}^{-1}$. The experimental studies have been completed and we are in the process of final data reduction.

In the coming year, we will use our measured vinylidene extinction coefficient to study the kinetics of the reactions of vinylidene with $\mathrm{H}_{2}$, $\mathrm{CH}_{4}$, and $\mathrm{N}_{2}$. In the case of $\mathrm{H}_{2}$ and $\mathrm{CH}_{4}$ the reactions are of great interest in planetary chemistry and will provide a check on our earlier theoretical calculations of these rate constants. For $\mathrm{N}_{2}$, the interest is in any reaction which can lead directly to the formation of $\mathrm{HCN}$.

7. Oxidation Chemistry

(R. I. Martinez)

The general objective of this program is to understand in detail the chemical kinetics of oxidation reactions, particularly those reactions which involve $\mathrm{O}, \mathrm{O}_{2}$, and $\mathrm{O}_{3}$.

Most of our efforts in the past several years have been directed towards a detailed understanding of the reactions of ozone with alkenes in the gas phase. Photochemical air poilution is driven by the oxidation of organic compounds, and the oxidation of alkenes by $\mathrm{O}_{3}$ is among the most important of all classes of atmospheric reactions. The kinetics and energetics of dioxymethylenes and their isomers, which we derived from studies on $\mathrm{O}_{3}$-alkene systems, led to our unifying postulate that the three reaction systems $R^{\prime} R^{\prime \prime} C:+O_{2}, \quad O+R^{\prime} R^{\prime \prime} C=0$, and $O_{3}+$ olefins proceed $v i a$ a common intermediate dioxymethylene $\left(R^{\prime} R^{\prime \prime} \dot{C} 00 \cdot\right\}$, i.e.,

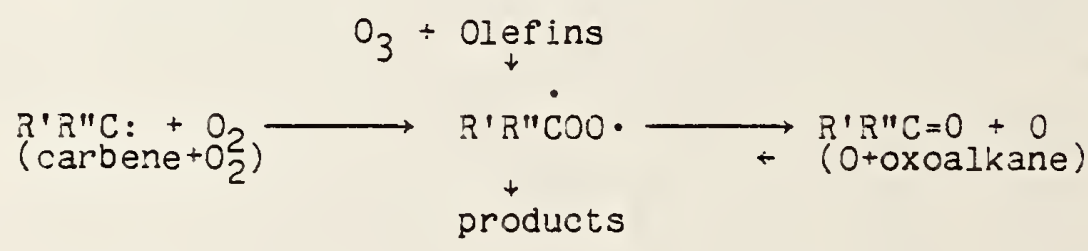

Additional support for this unifying postulate has been derived from studies on the photooxidation of formaidehyde and the reaction of $\mathrm{O}_{3}$ with tetramethyle thylene (TME). 
In the case of the reaction of ozone with tetramethylethylene (TME), temporal dependences of the products at 4 torr suggest that the process $\mathrm{Me}_{2} \mathrm{COO} \rightarrow \mathrm{Me}_{2} \mathrm{CQ}+\mathrm{O}$ accounts for 20-30 percent of the dissociative oranching of the $\mathrm{Me}_{2} \mathrm{COO}$ - produced via $\mathrm{O}_{3}+\mathrm{TME}$. It also suggests dissociative branching to $\mathrm{OH}$ to the extent of $\$ 5-10$ percent following the known intramolecular rearrangement of the $\mathrm{Me}_{2} \mathrm{COO}$. to the hydroperoxide $\mathrm{H}_{2} \mathrm{C}=\mathrm{C}\left(\mathrm{CH}_{3}\right)-\mathrm{O}-\mathrm{OH}$. At atmospheric pressure and in solution it has been found that the hydroperoxide further isomerizes to hydroxyacetone by intramolecular OH migration. It thus appears that at low pressures the intermediate hydroperoxide may lose the $\mathrm{OH}$ to some extent in competition with its isomerization to hydroxyacetone.

The photooxidation of formaldehyde plays an important role in diurnal photochemical smog cycles, and the elementary processes involved have been the focus of intense research in recent years. The unifying postulate above formed the basis for an extended version of our previously proposed mechanism for the photooxidation of formaldehyde. This mechanism provides a reinterpretation consistent with all observations to date. It involves photoisomerization of the $\mathrm{H}_{2} \mathrm{CO}$ to the hydroxymethylene $\mathrm{HCOH}$ which subsequently reacts with $\mathrm{O}_{2}$ to form the hydroxy-substituted dioxymethylene $\dot{H} \dot{C}(\mathrm{OH}) 00$. which subsequently decomposes to the products observed.

Dissociation of the $\dot{H} C(O H) O 0$ t to $O+H C(O) O H$ can be compared with the same process for the isoelectronic analogue $H \dot{C}(C l) 00 \cdot \rightarrow 0+H(C l)=0$ which accounted for ca. 20 percent of the dissociative branching of the $\mathrm{H} \dot{C}(\mathrm{Cl}) 00$. studied in collaboration with $\mathrm{Dr}$. Hiromi Niki of Eord Motor Co.

\section{Condensed Phase Kinetics}

1. Thermal Reactions of Aromatic Compounds

(S.E. Stein, L. Griffitins, M. Manka, R. Billmers and A. Fahr)

This program represents one of the main thrusts of our competence program. As in the gas phase, under severe conditions aromatic compounds are of key importance. Our work in this areas has led to a new and unexpected mechanism for anthracene pyrolysis. Rate constants for elementary reactions involving aromatic molecuiar and free racical species in Iiquids have been determined. Our ESR spectrometer has proved to be extremeiy valuable in providing a direct means of detecting radicals at high temperature. A database of kinetics information is being constructed and some significant general kinds have been observed. Our work on graphite lising self consistent field calculations have now covered systems up to 1000 carbon atoms. This is close to two orders magnitude larger than systems that have previcusly been studied by this method. Some extraordinarly suggestive insights on the effect $c I^{3}$ edges and resonance energies have been obtained.

\section{(a) Mechanistic Studies of Aromatic Pyrolysis}

We are examining reactions in organic pluids at high temperatures with the aim or both finding reaction pathways leading to polymerization and carbonization and using this information to develop predictive theory. Our continuing experimental and theoretical studies of 
the anthracene pyrolysis system has clearly demonstrated that dimerization occurs by a simple and entirely unexpected coupling of two ground state molecules described by the bimolecular rate constant $\mathrm{k} / 2 \cdot \mathrm{mol}^{-1} \cdot \mathrm{s}^{-1}=$ $10^{8.4} \exp (-45,200$ (cal)/RT). We propose a novel mechanism in which the following biradical intermediate,<smiles>CC1c2ccccc2[C@H](C)[C@@H]1C=Cc1ccc2ccccc2c1</smiles>

transfers an $\mathrm{H}$-atom Prom one anthracene unit to the other thereby forming a stable dihydro-diaryl compound. This mechanism suggests that larger polyaromatic molecules can form analogous biradicals of lower energy and therefore react even faster. This process provides a libiquitous means of initiating decomposition reactions of species that are otherwise unreactive. This latter point has been demonstrated in preliminary experimental studies of two other polyaromatic molecules, phenanthrene and pyrene.

During the next year we intend to determine detailed polymerization mechanisms for polyaromatic molecules other than anthracene in order to determine the generality of the above findings as well as to develop more general predictive models.

\section{(b) Elementary Kinetic Studies of Polyaromatic Species in Solution.}

These experiments are designed to yield rate constants and thermodynamics of elementary chemical reactions involving polyaromatic molecules and free radicals. Reactions are studied when they have been identified as key processes in the thermal chemistry of aromatic substances and their rate constants are not available or reliably estimatable.

The transfer of hydrogen from one species to another in Iiquid pyrolysis environments is a critical feature of many pyrolysis mechanisms. We have examined a number of such processes in detail. In one study, we made what we believe is the first direct measurement of the rate constant for H-atom transier from one closed-shell molecule to another. This orten proposed radical formation step was found to occur without complication in the following reaction, 


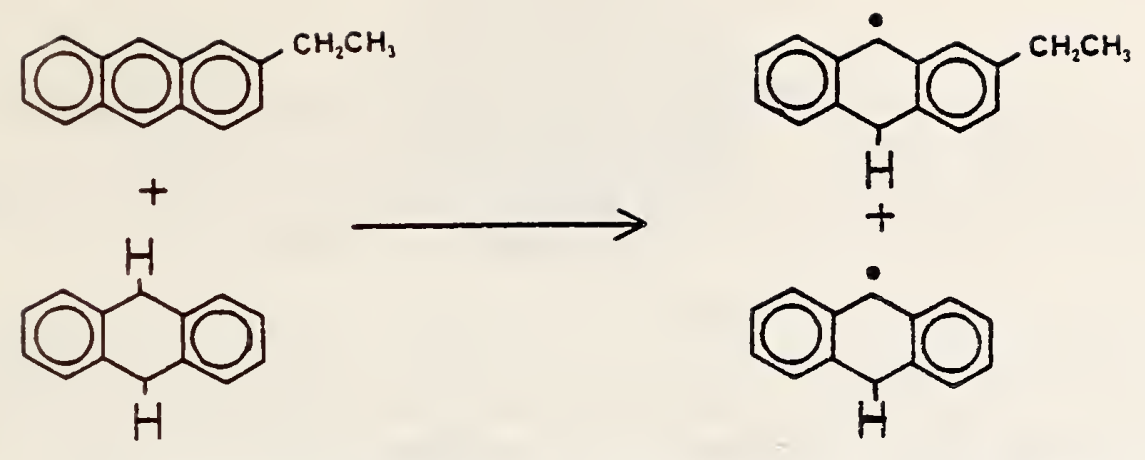

Because of the exceptionally low exothermicity of the reaction ( $-35 \mathrm{kcal}$ mol-i), side reactions and chain processes which normally obscure such processes did not occur. These measurements now enable us to place rate constants for a wide range of such reactions on an absolute basis.

In another series of studies we devised and used a new kinetic system for measuring rate constants for $\mathrm{H}$-atom abstraction by resonance stabilized radicals from aromatic molecules. Despite the significance of these reactions in pyrolysis chemistry, only one absolute measurement of a rate constant for such a reaction had been reported in the literature (namely $\left.\phi \mathrm{CH}_{2}+\phi^{1} \mathrm{CH}_{3} \rightarrow \phi \mathrm{CH}_{3}+\phi^{1} \dot{\mathrm{CH}}_{2}, \mathrm{k} / 2 \mathrm{~mol}^{-1} \mathrm{~s}^{-1}=10^{10.5} \exp (-11000 / \mathrm{T})\right)$. Our measurements were made in a reaction system involving. an equilioration between $R_{2}$ molecules and $R$. radicals $(R \cdot=\phi \phi$ and ). Addition of $\mathrm{H}$-donor molecules to these system opened up the desired reaction channel, namely $\mathrm{H}$-atom abstraction by $\mathrm{R} \cdot$, the rate of which could be measured by end product analysis. A series of $\mathrm{H}$-donors were studied and a strong correlation between reaction exothermicity and rate was found. For thermoneutral reactions, absolute rate constants were comparable to the above mentioned value for the benzyl radical. The most surprising observation was that these reactions have unusually high pre-exponential

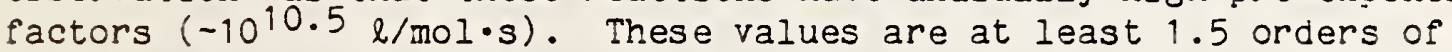
magnitude higher than expected on the basis of either transition state theory or gas-phase measurements for related reactions. The origin of this discrepancy is now under study.

Over the next year rates and mechanisms of hydrogen transfer reactions which transform one hydroaromatic structure into another (e.g.,

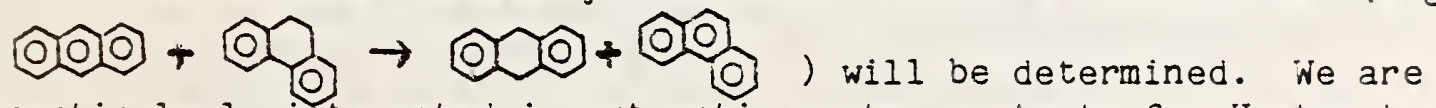
particularly interested in extracting rate constants for H-atom transfer from a radical to a molecule. These processes are of ten assumed to occur, but a rate constant for such a reaction has never been unambiguously measured.

2. ESR Detection of Aromatic Radicals at High Temperatures (R. L. Brown)

As part of a continuing attempt to measure high temperature equilibria involving aromatic radicals, the pyrolysis of xanthene (O) ) in benzene solution was Examined. The ESR spectrum of the rather stable xanthyl radical was easily observed over a temperature range of $250^{\circ}$ to $330^{\circ} \mathrm{C}$. A kinetic computer model of this reacting mixture was 
developed using a xanthyl dimer dissociation rate constant measured in this laboratory by other means. The xanthyl radical concentration at various temperatures predicted by this method was found to be in agreement with the concentrations observed in preliminary experiments.

The use of ESR in measuring aromatic radicals at high temperatures and pressures has been hampered by the low inherent sensitivity of this method. Measurements are limited to systems containing relatively stable radicals. In an attempt to circumvent this problem, we have begun to explore a different approach. An easily generated radical such as t-butoxy can be used to generate unstable radicals by hydrogen abstraction directly in the ESR cavity. From the observed radicai concentration and the concentration of dimer product it should be possible in favorable situations to measure hydrogen abstraction rates and radical recombination rates. To test the easibility of this technique a mixture of $t-b u t y l$ peroxide initiator in isopropyl alcohol was pyrolysed over a temperature range of 150 to $275^{\circ} \mathrm{C}$. A spectrum of hydroxyisopropyl radical was seen at $180^{\circ} \mathrm{C}$; it increased in intensity up to $215^{\circ} \mathrm{C}$, and then decreased, disappearing at $250^{\circ}$. The decrease at higher temperature can be attributed to the destruction of the peroxide initiator before the sample reached the ESR cavity. Since hydroxyisopropyl is not a particularly stable radical, this experiment provides encouraging support for this approach. In addition, by using the initiator with a mixture of two reactants, it may be possible to observe two different unstable radicals at the same time. This could make possible the measurement of radical-radical equilibria.

3. A Chemical Theory of Graphite

(R. L. Brown and S. E. Stein)

Graphitic materials can be asslimed to consist of loosely bound layers of very large planar polyaromatic molecules of varying size and having a variety of edge structures. Theories of aromatic molecules separate the bond joining the carbon atoms into two types; the o-bonds, which are localized in the region between the adjacent nuclei, and the $\pi$-bonds arising from the $\pi$-electrons which $l i e$ above and below the plane of the molecule. These $\pi$-electrons are metallic in nature and are the ones which give graphite most of its characteristic properties. There are a number of theories which have been successfully used to model such $\pi$-electron networks. However, they have been applied almost exclusively to the relatively small molecules of interest to organic chemists. Their application to very large graphitic molecules is the zoal of this program. Assistance for this research was provided by the Air Eorce orfice of Scientific Research.

Part of our work during the past year involved the systematic application of the simplest theories to the largest molecules possible. In addition to finding general structure/reactivity relations, this work provides a logical foundation on which to build more sophisticated theoretical analysis. Selected results of these calculations for $1 / 12$ "slices" of hexagonal molecules are shown in Figure 4. 


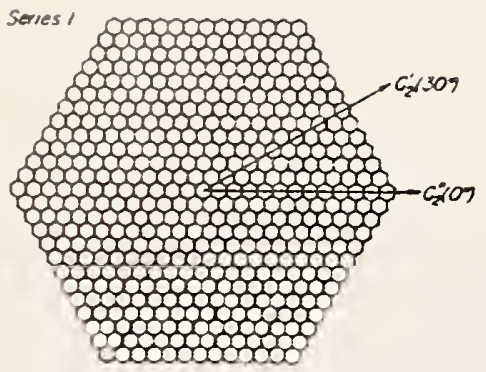

Series 2.

$1-90$ Series !,

1000

101000

olololo. olololololor ololololor-c3. (c) 10

toloó olololo ololololo 0010010010 00010000010 ololololol-

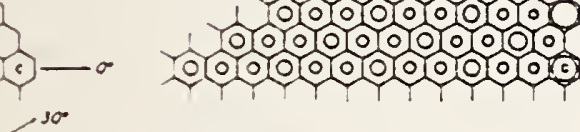
lololo lolololo
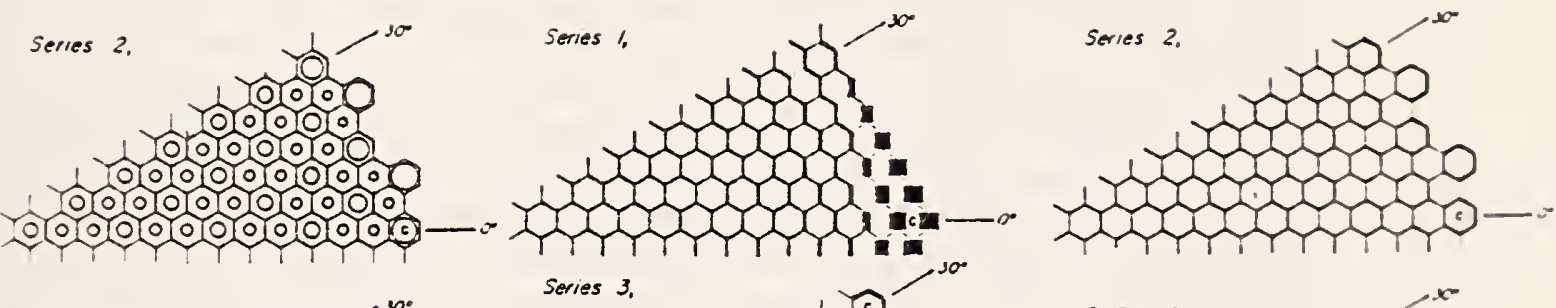

Series 4 ,

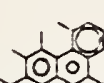

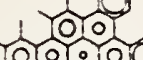
olololor ololololo

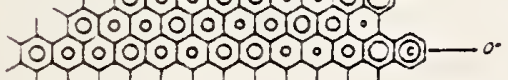
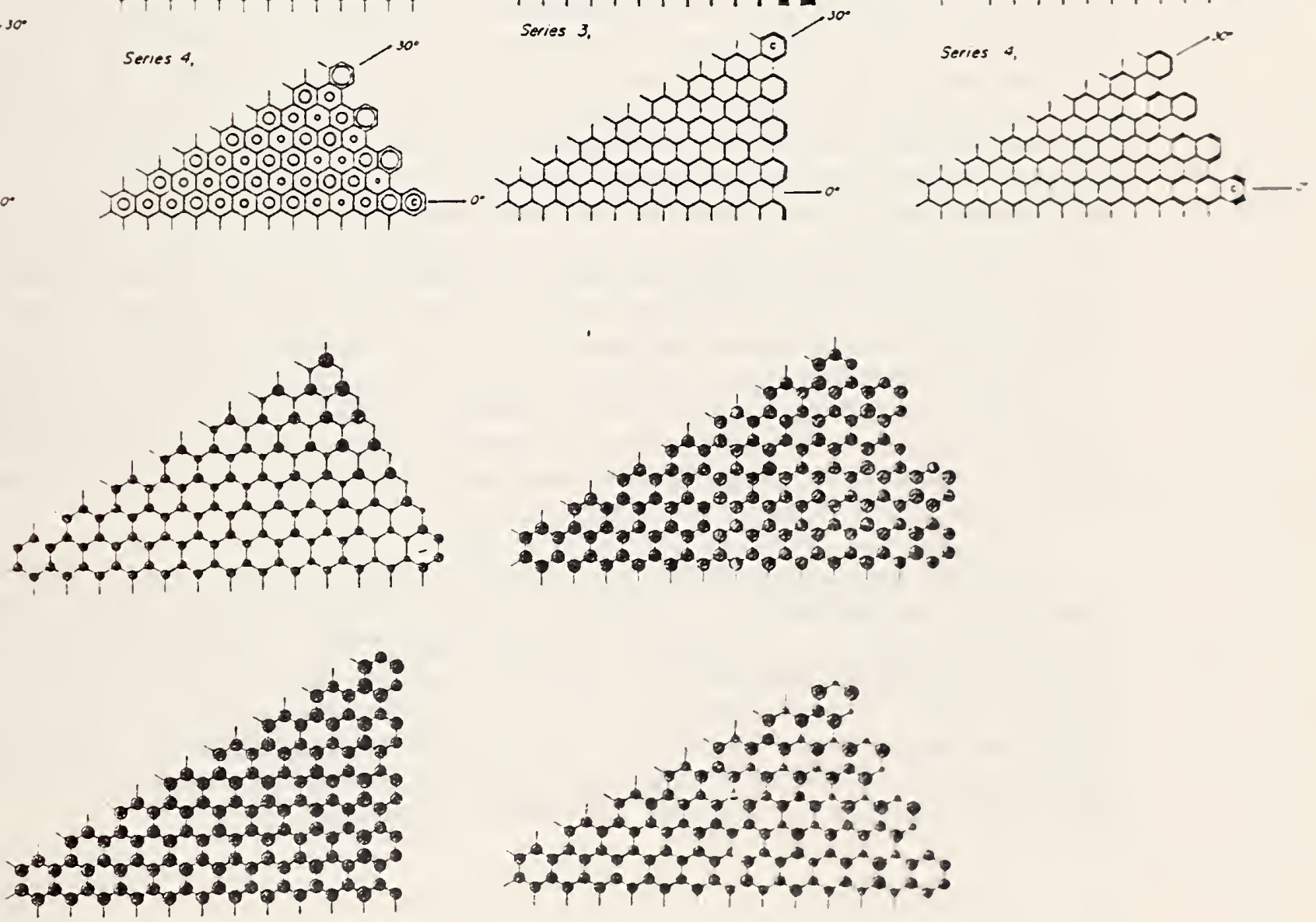

Fi3. 4. Properties of Graphite-Like Molecules. Results are shown as $i /: 2$ "slices" of hexagcnal molecules. Such a slice is shown ior a typical molecule in the top structure. Four different edge types were studied (series 1 to 4). The upper left set of :our structures show ring aromaticities - the area of each ring 13 proportional to its aromaticity. The upper right set show bond orders - the width of each bond is proportional to its bond order. The lower set shows localization enerstes - the area of each circle is proportional to the energy required to locallze an electron at the position of the circle. 
Much of our work over the past year was devoted to the development of efficient computer codes to adapt a sophisticated theory, namely the Pople SCF method, to very large molecules. All of the theories we have used require constructing a so-called bond matrix which describes how the carbon atoms are connected. For the simpler theories the key to their extension to large molecules was the discovery of a unique numbering system for the carbon atoms which results in a narrow banded bond matrix which greatly simplified the computations. The corresponding matrix for the SCF method, however, is not banded, and in practice contains few, if any, zero elements. Calculations using this type of matrix require far more computational time than the banded cases. Furthermore, the SCF method is iterative and converges more slowly the larger the molecule. Thus the computational problem rapidly becomes enormous for molecules of even modest size. The only way to use this method on large molecules is to require them to have the maximum symmetry. Then, group theoretical methods can be applied to greatly simplify the problem. Another advantage of using symmetrical structures is that they are easily formed into homologous series each consisting of a progression of increasingly larger molecules. This facilitated the extrapolation of various electronic properties to infinite size molecules (graphite) and the development of structure/reactivity relations at the "edges" of large polyaromatic molecules. By this means we have developed computer codes which allows us to use this SCF technique on molecules containing up to 1000 carbon atoms. For complete converzence of this iterative method, such large molecules require three to six days on a high speed computer. To our knowledge, the largest molecule to which this method had previously been applied was coronene, which contains 24 carbon atoms.

Our results so far with this more complex model are usually in qualitative agreement with simpler theories. One critical difference, however, is that SCF methods, in accord with intuition, indicate that resonance energies per C-atom approach a unique value with increasing molecular size, independent of edge iype. For the simplest theory used in previous studies, different limiting values were approached which depended on edge type. There are other general important qualitative differences whose implications we are now examining in detail. 
D. Brault, J. Butler, A. Harriman, R. E. Huie, Z. Karpas,

R. D. Levin, S. G. Lias, C. Lutz, M. Mautner, P. Neta,

H. Okabe, I. Price, M.-C. Richoux, F. F. Schwarz,

L. W. Sieck and C. Speller

\section{A. Introduction}

The scientific emphasis and focus of the work of the Ion Kinetics Group is derived largely from the competence-building program, "Physical and Chemical Effects of Ionizing Radiation on Matter", which is a joint effort with the Center for Radiation Research. The Ion Kinetics Group was organized in 1979 under the direction of Dr. P. Ausloos to fulfill the CCP component of the Competence. Originally called the Ionizing Radiation Group and assigned to the Chemical Thermodynamics Division, the nucleus of the group was transferred in 1983 to the Chemical Kinetics Division where the work on the kinetics of ionic processes became a natural complement to the existing programs on the kinetics of neutral species. The preexisting Chemical Kinetics Division program on Aqueous Chemical Kinetics was incorporated as an integral part of the Liquid Phase Ion Kinetics activities. The group also has been historically affiliated with the Ion Kinetics and Energetics Data Center, with members of the scientific staf: collaborating on compilations and evaluations of data necessary to the understanding of the chemistry of ions and the effects of ionizing radiation on matter. The activities of the Data Center are described in Section 2 .

The long range goal of the Competence on the Physical and Chemical effects of Ionizing Radiation on Matter is to obtain experimental and theoretical results which contribute to the understanding of the response of matter to ionizing radiation in sufficient detail for application to radiation biology, radiation dosimetry, and radiation processing.

The approach to the study of radiation effects involves diverse experimental tasks which examine individual facets of the problem area in detail. These individual tasks are summarized below:

Energy Deposition

Experiments aimed at achieving a fundamental understanding of the initial ionization event.

Radiation-Induced Frazmentation of Organic and Biochemical Ions

Stucies of the energetics, rates and modes of fragmentation of ions.

Kinetics of the Interactions between Ions and Molecuies

Fundamental investigations of the chemistry of ions, including elucidations of reaction rates and mechanisms, thermochemistry. 
Energetics of Solvation and the Chemistry of Solvated Ions

Studying the link between the kinetics and thermochemistry of lons in the gas phase and in the liquid phase; development of predictive capacity to apply results of gas phase mass spectrometric studies to condensed phase systems.

Charge Recombination of Ionized Transients

Providing insight into energy transfer and radiation damage mechanisms.

Radiation Chemistry of Organic and Biochemical Solutions

Energy transfer, radiation damage in biologically important substances.

Kinetics and Radiation Chemistry of Aqueous Inorganic Solutions

Electron transfer reactions in aqueous solutions of inorganic salts, particularly emphasizing the kinetics of reactions involving $\mathrm{SO}_{3}{ }^{-}, \mathrm{SO}_{5}{ }^{-}$, and $\mathrm{HSO}_{5}{ }^{-}$.

Ion Kinetics and Energetics Data Center

Building and maintaining up-to-date evaluated data base relating to ionization processes and lonic reactions.

The description of the scientific activities of the Group which follows will be organized under headings corresponding to the various activities listed above, although an arbitrary subdivision has been made between Gas Phase Ion Kinetics and Liquid Phase Ion Kinetics. Data Center activities are discussed in Section 2. It will be noted that there is considerable overlap between the various areas of activity, with many individuals contributing to more than one task.

B. Gas Phase Ion Kinetics

The program aims at achieving a fundamental understanding of the unimolecular and bimolecular reactions of ionic species generated by high energy radiation, and further, at examining the effects, of ion-molecule clustering, i.e., of solvation. Aspects of the program currentiy supported by the Department of Energy and the Air Force Office of Scientific Research give particular emphasis to examinations of processes involving small organic molecules, while experimental work tied in to the liquid Phase Ion Kinetics effort (described below) focuses on model compounds which give information about processes in biochemical systems. 
1. Radiation-Induced Fragmentation of Organic Ions

( $J$. Butler, in collaboration with $R$. Stockbauer, 541 , and $A$.

Paar, CRR; S. G. Lias, L. W. Sieck)

The overall objective of this research is to provide a detailed understanding of the disposal of energy by the excited ions wich initially result from the absorption of ionizing radiation, and to determine the kinetic and thermodynamic parameters which influence the fragmentation processes.

Results obtained during the past year include:

(a) A determination of the energetics of the reaction:

$$
\left(\text { tert- } \mathrm{C}_{4} \mathrm{H}_{9}\right) \mathrm{C}_{6} \mathrm{H}_{5}^{+} \rightarrow \mathrm{C}_{6} \mathrm{H}_{5} \mathrm{C}\left(\mathrm{CH}_{3}\right)_{2}^{+}+\mathrm{CH}_{3}
$$

which led to an accurate value for the heat of formation of the isopropylbenzene (cumyl) ion. This is the same ion which is formed by the protonation of $\mathrm{C}_{6} \mathrm{H}_{5} \mathrm{C}\left(\mathrm{CH}_{3}\right)=\mathrm{CH}_{2}$, and therefore, the experimental result provided a comparison standard for the evaluation of the scale of proton affinities completed by the Ion Kinetics and Energetics Data Center last year. This result was obtained from photoelectron-photoion coincidence experiments, in which ions of known excitation energy are sampled by examining those positively-charged species formed in coincidence ' $i$ ith zero energy electrons, where the ionization event was effected by photons of known energy. The experiments also yield rate constants and entropy changes associated with the dissociation processes, as well as accurate heats of formation of the fragment ions.

(b) In order to evaluate information about the structures and thermochemistry of ions isomeric to protonated benzene the fragmentation processes leading to the formation of this ion were studied in 1,3,5hexatriene, 1,3- and 1,4-cyclohexadiene using the photoelectron-photoion coincidence technique. It was found that the slow fragmentation process:

$$
\mathrm{C}_{6} \mathrm{H}_{8}^{+} \rightarrow \mathrm{C}_{6} \mathrm{H}_{7}^{+}+\mathrm{H}
$$

proceeds in all three systems through the same cyclic transition 3 tate, which lies at an energy level higher than the $\left(\mathrm{c}-\mathrm{C}_{6} \mathrm{H}_{7}^{+}+\mathrm{H}\right)$ products.

Figure 5 snows the normalized abundances of the parent $\mathrm{C}_{6} \mathrm{H}_{8}$ ions and the fragment $\mathrm{C}_{6} \stackrel{+}{H}_{7}$ ions formed in the three subject compounds as a function of enerzy, examined after 1.2 microseconds and 6.2 microseconds. The bimolecular chemistry of $\mathrm{C}_{6} \mathrm{H}_{7}$ ions was also investigated. 

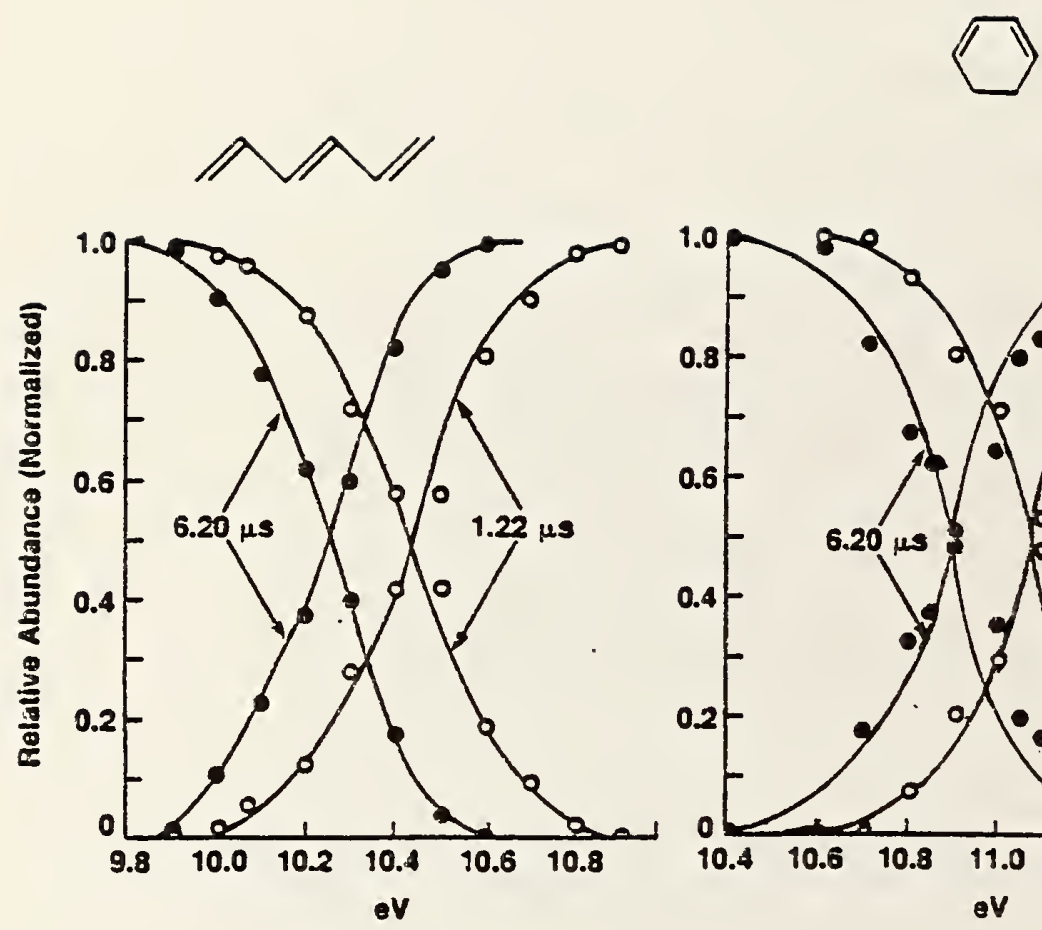

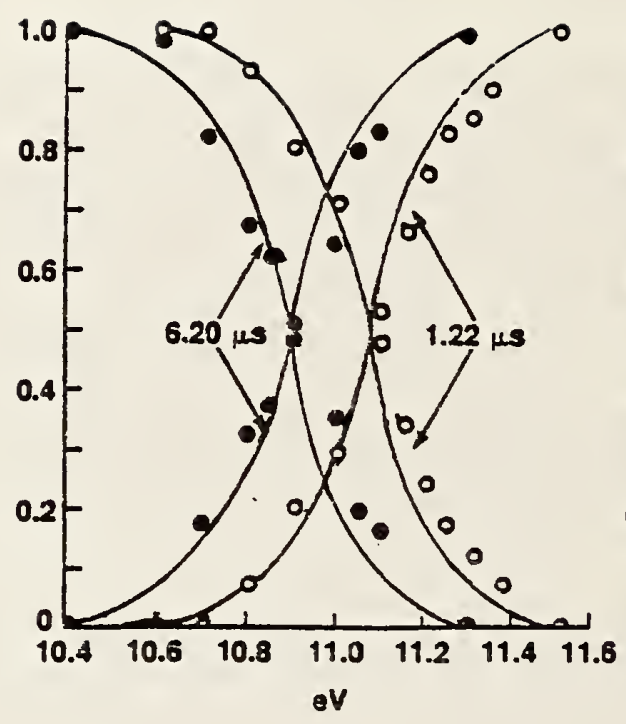

Fig. 5. Photoelectron-photoion coincidence results elucidating the fragmentation process: $\mathrm{C}_{6} \mathrm{H}_{8}^{+}+\mathrm{h \nu}+\mathrm{C}_{6} \mathrm{H}_{7}^{+}+\mathrm{H}$ in the $\mathrm{C}_{6} \mathrm{H}_{8}$ isomers 1,3,5-hexatriene, 1,3-cyclohexadiene, and 1,4-cyclohexadiene. Shown are the normalized relative abundances of the parent $\mathrm{C}_{6} \mathrm{H}_{8}{ }^{+}$ ions (decreasing curves) and daughter $\mathrm{C}_{6} \mathrm{H}_{7}{ }^{+}$ions (increasing curves) observed in coincidence with zero-energy electrons, plotted as a fu nction of the energy, of the ionizing photons. The ions are observed after $1.22 \mathrm{~s} \times 10^{-6}$ and $6.20 \mathrm{~s} \times 10^{-0}$. In all three cases, the degree of fragmentation observed is greater after $6.20 \mathrm{~s} \times 10^{-6}$ than after $1.22 \mathrm{~s} \times 10^{-6}$, demonstrating that the fragmentation processes are slow. RRKM modelling of the fragmentation (solid lines) demonstrates that the dissociation processes in the three systems all proceed through the same cyclic transition state. 
(c) Other work carried out to provide benchmark measurements for evaluations of ion thermochemistry included a study of ionic fragmentation processes in dimethylether. An accurate heat of formation for the $\mathrm{CH}_{3} \mathrm{OCH}_{2}$ ion was obtained. It was also demonstrated in a photoion-photoelectron coincidence study that the $\mathrm{HCO}^{+}$ion formed in dimethylether results both from dissociation of $\mathrm{H}_{3} \mathrm{CO}^{+}$and of $\mathrm{CH}_{3} \mathrm{OCH}_{2}$.

(d) A detailed evaluation was made of thermal activation efiliencies at the low pressure limit of the decomposition of protonated methoxyethers for a variety of organic and inorganic bath gases. The measurements, made on the high pressure pulsed electron mass spectrometer, provide the only data available on thermal activation for ionic systems in the vapor phase. Although the ordering of efficiencies is qualitatively similar to that generally found for vibrational activation of nelitrals, the spread in values is greater for the ionic system. The work was sponsored by the Department of Energy.

Future plans include the examination of ionic eragmentation mechanisms in styrene and cyclooctatetraene which will resolve conflicting information in the literature about heats of formation of ions, and aid in the evaluation of data for a forthcoming Data Center evaluation effort. Plans also include the determination of ionic fragmentation energetics in phenylacetylene, which will tie in with an effort described below in which the ion-molecule kinetics in this system will be elucidated.

2. Kinetics of the Interactions between Ions and Molecules (S. G. Lias, P. Ausloos, M. Mautner, T. Buckley, L. W. Sieck, F. Schwarz)

Irradiation at energies above the ionization threshold produces positive ions which may undergo rapid ion-molecule reactions. This task involves the elucidation of the bimolecular chemistry of ions, including an examination of reaction mechanisms, and measurements of rate constants and equilibrium constants as a function of temperature. The latter experiments lead to quantitative information about the thermochemistry of proton transfer, charge transfer, and other ionic reactions. Much of the work ties in directly with the above-described task or ionic fragmentation processes, as well as with the work of the Ion Kinetics and Energetics Data Center. Accomplishments of the past year are discussed beiow.

(a) In conjunotion with the Data Center evaluation of the thermochemistry of ions, questions arose about the structure(s) and possible isomerization mechanisms of ions of the empirical formula $\mathrm{C}_{5} \mathrm{H}_{7}$. In most systems where $\mathrm{C}_{6} \mathrm{H}_{7}$ species occur, it has previously been assumed that the ions will predominantly have the thermodynamically most stable structure, that of protonated benzene. A study was made of the reaction kinetics of this important species originating (a) from eragmentation of parent radical cations ir 1,3,5-hexatriene, 1,4- and 1.3-cyclohexadiene, and a variety of $\mathrm{C}_{6} \mathrm{H}_{10}$ isomers or (b) in ion-molecule reactions such as:

$$
\mathrm{C}_{3} \mathrm{H}_{4}^{+}+\mathrm{C}_{3} \mathrm{H}_{4} \rightarrow \mathrm{C}_{6} \mathrm{H}_{7}^{+}+\mathrm{H}
$$


(where $\mathrm{C}_{3} \mathrm{H}_{4}$ is allene or propyne). It was demonstrated that in each of these systems, the ion exhibits at least two structures, and the second, non-benzenium $\mathrm{C}_{6} \mathrm{H}_{7}$ structure is different in different systems. Probable structures for the other isomers are protonated fulvene (protonated methylenecyclopentadiene) and protonated dimethylenecyclobutene.

(b) In molecules which undergo large geometry changes upon ionization, ionization energies are difficult to determine by techniques which detect the onset of ionization as a function of energy; in such cases the onset of ionization is a slowly increasing function of energy and the exact onset energy is difficult to pinpoint. For this reason, the adiabatic ionization energies of hydrazines have never before been determined successfully. In work carried out during the past year, the ionization energies of a variety of substituted hydrazines were determined in the pulsed nigh pressure mass spectrometer by measuring the equilibrium constants of charge transfer reactions:

$$
\mathrm{A}^{+}+\mathrm{B}+\mathrm{B}^{+}=\mathrm{A}
$$

(where $A$ is a hydrazine and $B$ is a reference compound with a well-established ionization energy). The enthalpy change of the charge transfer reaction is the difference in the adiabatic ionization potentials of $B$ and $A$. The effects of steric constraints on ionization energies in these compounds was also examined.

Similar experiments carried out in the ion cyclotron resonance spectrometer resulted in the first determination of the adiabatic ionization energy of cyclopropane, which undergoes a Jahn-Teller distortion upon ionization.

(c) As an adjunct to an extensive evaluation of proton af $f$ inity data completed during the past year in the Ion Kinetics and Energetics Data Center (Section 2), a large number of proton affinity determinations for species of special interest or importance have been carried out through measurements of equilibrium constants of proton transfer reactions using both the pulsed high pressure mass spectrometer and the ion cyclotron resonance spectrometer.

(d) In a project sponsored by the Air Force Office of Scientific Research, the rates and mechanisms of consecutive ion-molecule condensation reactions in diacetylene, cyancacetylene, and cyanogen have been examined in both the ion cyclotron resonance spectrometer and the pulsed high pressure mass spectrometer. These compounds, which contain conjugated triple bonds undergo efficient condensation and condensation-dissociation sequences leading to the formation of high molecular weight hydrogen-deficient ions such as $\mathrm{C}_{18} \mathrm{H}_{8}$ in diacetylene. The ion cyclotron resonance instrument was modified so that the trapped ions could be irradiated by photons from a pulsed dye laser, and the photodissociation mechanisms of the product condensation ions were mapped. 
(e) The reactions of radical cations in excited electronic states have been investigated using pulse radiolysis to generate the exclted ions, and fluorescence measurements to follow the abundances of the excited lons as a function of time. Fluorinated benzene ions emit in the visible $(470 \mathrm{~nm})$ with appreciable yields. In the presence of excess krypton, argon, neon, or helium, the charge transfer from the inert gas,

$$
\mathrm{X}^{+}+\mathrm{C}_{6}(\mathrm{H}, \mathrm{F})_{6} \rightarrow \mathrm{X}+\mathrm{C}_{6}(\mathrm{H}, \bar{F})_{6}^{+*}
$$

(where $X$ is the rare gas) produced the fiuorescing cation; this reaction can not be studied by any other technique. The results yield rate constants for this ion-molecule reaction which are close to the ion-molecule collision rates.

Future plans include additional work on the ionization energies of hydrazines which will examine such effects as rigid geometry, constrained angles and bulky substituents on ionization energies. In this group, the effects of the large geometry changes on the kinetics of electron transfer will be also be investigated. Preliminary results show that the reactions are unusually slow for exothermic charge transfer processes. In addition, a comprehensive study of ion-molecule collision kinetics including highly accurate determinations of the rate constants for collision-rate processes in a variety of organic compounds will be made. We will also investigate the kinetics of the reactions of the phenyl ion and other $\mathrm{C}_{6} \mathrm{H}_{5}^{+}$1somers. The chemistry of this important species, which is generated in irradiated aromatic compounds, has never been systematically explored. Studies will be made of ion-molecule mechanisms in aromatic molecules with unsaturated side chains.

\section{Solvation Thermodynamics and the Kinetics of Solvated Ions} (M. Mautner, L. W. Sieck, S. G. Lias, and C. Speller)

Ionic species, such as those generated in acidic or basic envircnments or by radiation are ubiquitous intermediates in biochemical and organic reactions. The stabilities and reactivities ol ionic intermediates are affected by their intrinsic molecular properties (studied in the gas phase under tasks (a) and (b), above), and by interactions with the condensed phase environment. This task utilizes the high pressure mass spectrometer to bridge the gap between the understanding of ionic chemistry attained in the gas phase studies and that required for understanding and prediction of processes occurring in irradlated liquids, acid-catalyzed reactions, and enzyme catalyzed processes. In this instrument, it is possible to generate a given ion in the presence of a solvation species (such as $\mathrm{H}_{2} \mathrm{O}$, for example), to determine the thermochemistry of solvation by an examination of equilibria such as:

$$
\begin{gathered}
A^{+}+M \rightarrow\left(A^{+} \cdot M\right) \\
\left(A^{+} \cdot M\right)+M \rightarrow\left(A^{+} \cdot M 2\right) \text {, etc. }
\end{gathered}
$$


and to study the kinetics and mechanisms of reactions of the solvated ions.

Work has been carried out during the past year on the following problems :

(a) A comprehensive investigation was conducted of the ionic hydrogen bond, which plays a dominant role in ion-solvent interactions. For structurally simple organic species, it was shown that the energy of the ion-solvent association bond is directly proportional to a molecular property, the proton affinity of the molecule. The proportionality constants for a great variety of $A \cdot \cdots H^{+} \cdot \cdots B$ association ions (where $A$ and $B$ represent heteroatoms in different classes of molecules) have been generated in this laboratory. Such correlations can be used in estimations of cluster ion stabilities from proton affinity data. The strengths of ionic hydrogen bonds involving complex molecules were also determined. The results revealed structural effects such as hydrogen bonds between parts of the same molecule, as well as the occurrence of multiple bonding between ions and large molecules.

Further studies of this type sponsored by a contract with the Department of Energy were concerned with establishing the thermochemistry of hydration of low molecular weight sulfides and mercaptans, as węll as the hydrogen bond strengths associated with the solvation of $\mathrm{CH}_{3} \mathrm{NH}_{3}$ by several mercaptans $\left(\mathrm{N} \cdot \cdot \mathrm{H}^{+} \cdot \mathrm{SS}\right.$ and $\mathrm{S} \cdot \cdots \mathrm{H}^{+} \cdot \mathrm{S}$ S interactions). This data on hydration energies was used to correlate the relationship between the difference in proton affinity between $\mathrm{H}_{2} \mathrm{O}$ and any mercaptan as a function of the dissociation energy (stability) of the proton-bound hydrate, for use in estimating properties of other clusters, as described above.

(b) An interpretation of the observed correlation between proton affinity and the energy of ion-molecule association was provided by a study carried out on the ion cyclotron resonance instrument. The $H / D$ exchange processes:

$$
\mathrm{AH}^{+}+\mathrm{BD} \rightarrow[\mathrm{AH} \cdot \mathrm{BD}]^{+} \rightarrow \mathrm{AD}^{+}+\mathrm{BH}
$$

were examined for some of the association ions, [AH・BH], whose thermochemistry was determined in the high pressure mass spectrometer (see (a) above). It was shown that (a) the mechanism of the exchange reaction involves the occurrence of an endothermic proton transfer reaction in the [AH・BD] complex; (b) just as there is a relationship between the proton afinity of $\mathrm{AH}$ and the strength of bonding with $\mathrm{BH}$ (see (a)), the probability of occurrence of the $H / D$ exchange also correlates with the strength of bonding; (c) both effects can be interpreted in terms of a double-welled potential surface, the major features of which can be predicted simply from the classical ion-molecule interaction potential.

(c) The work described under (a) and (b) was concerned with systems in which the molecules contain atoms with free electron pairs which interact with a proton, i.e. systems with interactions of the types $\mathrm{N} \cdot \cdots \mathrm{H}^{+} \cdots \mathrm{O}, \mathrm{N} \cdots \mathrm{H}^{+} \cdots \mathrm{N}, 0 \cdots \mathrm{H}^{+} \cdots \mathrm{O}, \mathrm{S} \cdots \mathrm{H}^{+} \cdots \mathrm{O}$ and $\mathrm{N} \cdots \mathrm{H}^{+} \cdots \mathrm{S}$. However, 
In some systems, the results indicated that the proton interacts with a carbon atom or a carbon-based electron system. In this report period, extensive studies were dedicated to these types of unconventional hydrogen bonds, using as reactant quarternary ammonium ions for which only this type of interaction is possible. Such interactions were found to be unexpectedly strong, in the range of $40-80 \mathrm{~kJ} / \mathrm{mol}$. The results suggest that in solution, hydrogen atoms from solvent molecules may bond not only to a proton in the ionic species, but also to hydrocarbon chains or aromatic rings, which were thought not be significantly interactive. The results are pertinent to understanding interactions in biological systems, especially to the interactions of the quarternary neurotransmitter ion acetylcholine with neuroreceptors. The model developed also implies that interactions of amide groups with phenyl rings in proteins may play a role in peptide conformation.

(d) Rocket-born experiments have indicated the occurrence of tropospheric ion clusters which involve molecules of water, hydrogen cyanide and acetonitrile. Clusters of this kind can be generated in the high-pressure mass spectrometer. The stabilities of one of the major type of clusters, those containing only water and acetonitrile, were measured. The results are relevant not only to ionospheric chemistry, but also to the solvation of the proton in mixed solvents, such as the water-acetonitrile system, which is commonly used in liquid chromatography.

(e) The pulsed high pressure mass spectrometer was converted to negative ion operation in early 1984. The positive ion capability was retained, and switching between the two modes of operation can be completed in less than one minute using external circuitry only.

There is at present a striking lack of information about the association and clustering reactions of negative ions. This is particularly true for association ions incorporating relativeiy complex organic molecules. Such information is of fundamental scientific importance as well as impacting directly on negative chemical ionization analytical mass spectrometry, which utilizes association (adduct) ion formation for diagnostic purposes bcth in traditional mass spectrometric and tandem MS/MS instrumentation. Determination of the stabilities of association ions formed by the interactions of $\mathrm{Cl}^{-}$and $\mathrm{NO}_{2}^{-}$with a variety of organic molecules will be carried out. The $\mathrm{NO}_{x}^{2}$ type anions are easily generated in mixtures containing trace quantities of nitroalkanes, and they may provide a viable alternative to other reagent ions such as $\mathrm{C2}^{-}$, which is commonly used in negative chemical ionization analysis.

In the future the bonding in ionic charge transfer complexes will be examined. Of particular interest is the contribution of resonance charze transfer between components of the complex, and the dependence of rescnance contributions on moleclilar parameters such as ionization potentials and steric hindrarce. Also clustering studies will be extended to further clusters of ionospheric interest, and also to clusters where unconventional hydrogen bonds may occur. 
4. Charge Recombination of Ionized Transients

(F. Schwarz, H. Okabe, P. Ausloos)

In systems under high energy irradiation, the ions formed ultimately recombine with electrons or negative ions. In recent years, this Group has examined in some detail the kinetics and chemical consequences of ion-electron recombination in organic liquids ("geminate recombination"). During the past year, attention has been given to such processes occurring in the gas phase. Systems were selected for study in which the excited neutral species formed in the recombination:

$$
M^{+}+e N \rightarrow M^{*}
$$

dissociates to give excited fragments which fluoresce:

$$
M^{*} \rightarrow F^{*}+R \rightarrow F+R+h v
$$

In such systems the electron-ion recomination process can be followed by monitoring the emission intensity as a function of time.

This past year fluorinated methane and ethane gases were exposed to 3 nanosecond pulses of high energy $(0.6 \mathrm{MeV})$ electrons from a Febetron 706 , and the lifetime and spectrum of the resulting emission from excited iragments were monitored. In $\mathrm{CF}_{2} \mathrm{H}_{2}, \mathrm{CF}_{2} \mathrm{HCl}, \mathrm{CF}_{3} \mathrm{H}, \mathrm{CF}_{3} \mathrm{Cl}$, and $\mathrm{C}_{2} \mathrm{~F}_{6}$, the dissociative process following recombination could be established by examining the fluorescence of excited fragment species, i.e., in $\mathrm{CF}_{2} \mathrm{H}_{2}$ and $\mathrm{CF}_{2} \mathrm{HC} \&$ the recombination emission results from excited $\mathrm{CH}_{2}$ fragments, while $\mathrm{CF}_{3} \mathrm{H}, \mathrm{CF}_{3} \mathrm{Cl}$, and $\mathrm{C}_{2} \mathrm{~F}_{6}$ produce $\mathrm{CF}_{3}$ emission. Quenching constants of the fluorescence by various gases were also obtained. The only previous exploitation of recombination fluorescence as a means of monitoring ion-electron recombination in the gas phase was in inert gases at high pressure.

C. Liquid Phase Ion Kinetics

1. Radiation Chemistry of Organic and Biochemical Solutions (P. Neta, M.-C. Richoux, D. Brault, A. Harriman, and M. Mautner)

Pulse radiolytic techniques are used to characterize the chemical properties of short-lived species in irradiated solutions. Particular attention is given to reactions of reactive transients which have been implicated as intermediates in radiation-induced and thermal processes in a variety of chemical and biological systems. An important aspect of this program is the study of inter-and intramolecular electron transfer processes aimed at understanding similar phenomena in biological systems as well as in photochemical systems of interest for possible use in solar energy conversion; this aspect of the work is supported by the Department of Energy. By studying the kinetics and mechanisms of the various transient species and by understanding the parameters that control them, we can hope to be able to predict the behavior of these species in more complex systems. 
(a) The toxicity of halogenated hydrocarbons (such as carbon tetrachloride and the anesthestic agent halothane, $\mathrm{CF}_{3} \mathrm{CHC} 2 \mathrm{Br}$ ) is believed to originate from cytochrome-P 450 -mediated generation of peroxyl radicals with subsequent attack on biological targets, especially unsaturated lipids. The feasibility of this proposed mechanism was demonstrated in a pulse radiclytic study of the reactions of peroxyl radicals with iron porphyrins and with fatty acids. FeIII-deuteroporphyrin was used as a model for cytochrome $P_{450}$ and its reactions with peroxyl radicals were studied by pulse radiolysis. Peroxyl radicals derived from the halogenated hydrocarbons were iound to oxidize the ferric porphyrin by an electron transfer mechanism to produce a FelII-porphyrin pi-radical cation. The rates of reactions of oleic acid, linoleic acid, linolenic acid, and cholesterol with halogenated peroxyl radicals were much slower than the rates for reaction with the porphyrin. Furthermore, the peroxyl radicals derived from these fatty acids react with the porphyrin rapidly. The latter reaction leads to degradation of the cytochrome $P_{450}$ but is not a termination reaction, since it produces hydroperoxides which may, in turn, produce additional radicals to attack the lipids.

(b) Photogeneration of oxygen from water is an integral part of green plant photosynthesis and many laboratory models aimed at the storage of solar energy have tried to mimic this natural process. In this problem area, an experimental examination of the catalytic oxidation of water to oxygen by metalloporphyrin pi-radical cations has been carried out. In the ideal case, a metalloporphyrin (synthetic analogue of chlorophyli) absorbs the light and forms an excited state which then donates an electron to another compound in the system. The electron acceptor then reacts with a catalyst to form hydrogen from water. The metalloporphyrin radical-cation (formed after donating an electron) should react with another catalyst that will produce oxygen from water. The overall effect should be splitting of water to hydrogen (a fuel) and oxygen by sunlight with no net change in the otiner components of the system. For this purpose, it is necessary to find the proper catalyst, the best metalloporphyrin, and the optimal experimental conditions for oxygen production. This was done using pulse radiolysis and monitoring the rate of reaction of various porphyrins and catalysts to determine the factors that influence these rates. With this approach, a tedious search for oxygen production under different conditions was unnecessary. After optimization of the kinetic factors by pulse radiolysis, it was found that the optimal kinetic conditions also gave the highest cxygen yield.

The best porphyrin found for this purpose is not the cne predicted on a thermodynamic base to be the strongest oxidant (ZnTMPy?), but rather that which forms a Ionger-lived radical-cation (ZnTPPS). The best catalyst was found to be colloidal $\mathrm{RuO}_{2}$, and the rate of reaction increased with $\mathrm{pH}$, especially at $\mathrm{pH}>9$.

In conjunction with these studies, it was found that the radical cations produced by one-electron oxidation of porphyrins in aqueous solutions tend to form complexes with any anions present in solution. 
This complexation affects both the absorption spectra and the lifetime of the radical cation (and thus its ability to participate in oxygen production via the catalyst).

(c) During the past year, the program of study of intramolecular electron transfer processes, supported by the Department of Energy, was expanded to include investigations of temperature effects on such processes. The studies were carried out on nitroaromatic halides in which electron attachment on the nitro group is followed by electron transfer through the molecule leading to halide loss. Earlier work had demonstrated that the rate of the intramolecular electron transfer depends on the respective geometries and the relative electron afinities of the nitro and halide functions in the molecule. Since the dependence oi the rate constants on molecular geometry is governed by energy barriers and entropies of activation, a physical understanding of structural effects can be achieved only if both of these factors are known, i.e., if the rate constants are known as a function of temperature. A temperature-controlled pulse radiolysis cell was constructed and a series of reactions were measured. The results show that both the energy barriers and the entropies of activation vary significantly froin reaction to reaction. Some specific structural trends emerge, such as the decrease of reaction probabilities when steric pactors interfere with optimal orientation of the substituents for electron transfer.

The studies of intramolecular electron transfer processes have recently been extended to include additional types of compounds, namely covalently-bound dimers of metalloporphyrins ( $\left.M P-M^{\prime} P\right)$, Viologens $\left(V-V^{\prime}\right)$, and mixed dimers $(M p-V)$, zs well as related compounds with two reducible metal centers. The purpose of this work is to achieve an understanding of similar natural processes, occurring for example during photosynthesis, with the hope of syrthesizing a model that will mimic the natural process effectively.

In order to determine the enthalpy and entropy changes that contribute to the electron affinities of organic and biological molecules in aquecus solution, the temperature dependences of charge transfer equilibrium constants in the pulse radiolysis of liquids was studied using the temperature-controlied pulse radiolysis cell. Preliminary results show that the major differences between molecular electron affinities are due to enthalpy effects, but in large molecules entropy effects are also significant. This is of interest to biological systems related to the action of quinone containing co-enzymes, which are large molecules and which participate in biological electron transfer processes.

Planned extensions of this work include the study of reactions of peroxyl radicals with iron porphyrins and fatty acids, aimed at examining the factors causing the toxicity of halogenated hydrocarbons. This study will be continued with similar experiments on other possibly harmful halogenated hydrocarbons, such as other anesthetic agents and the pesticide ethylene dibromide. The reactions of the alkyl and perioxyl radicals derived from such compounds with iron porphyrins in their various oxidation states will be examined. 
In addition, the study of the catalytic oxidation of water to oxygen by metalloporphyrin pi-radical cations (aimed at mimicking the photogeneration of oxygen from water in natural photosynthesis) will be continued with an examination of the behavior of the cation radicals of several other porphyrins, in which either the ligand or the metal will be varied. Thus, the structural parameters which govern the process will be systematically examined. In conjunction with these experiments, the behavior of those porphyrins in reduction processes will also be studied, since recent results have demonstrated that the radical anion lifetimes vary over a very wide range, depending on the ligand and particularly on the central metal ion.

The temperature dependences of charge transfer equilibria in irradiated organic liquids will be determined for a large group of quinones and nitroaromatic molecules. The enthalpy and entropy changes and the resulting effects on molecular electron affinities, as well as the effects of alkyl, aryl and halogen substitution on these factors will be investigated. This work may be also extended to include acid-base dissociation equilibria. Exploratory investigations will be carried out on charge transfer through macromolecules. Specifically, the feasibility of measuring charge transfer through pulse irradiated polymer-coated electrodes will be explored. The studies of intramolecular electron transfer processes have in covalently-bound dimers of metalloporphyrins (MP-M'P), Viologens ( $\left.V-V^{\prime}\right)$, and mixed dimers $(M p-V)$, and related compounds with two metal centers will be expanded to examine the effects of molecular structure on the kinetics of these processes.

2. Kinetics and Radiation Chemistry of Aqueous Inorganic Solutions (R. E. Huie (in collaboration with Dr. N. C. Peterson of Polytechnic Institute of N.Y.) and P. Neta)

The current experimental work in aqueous phase chemical kinetics is an outgrowtin of an effort to understand the induced oxidation of iron by $\mathrm{SO}_{2}$ in aqueous solution. In this system, $\mathrm{SO}_{2}$ and ferrous ion are simultaneously oxidized under conditions such that neither would be oxidized alone. As described in last year's annual report, the mechanism previously proposed to explain this phenomenon has the radical anions $\mathrm{SO}_{3}^{-}$ and $\mathrm{SO}_{5}^{-}$as key intermediates, along with $\mathrm{HSO}_{5}^{-}$, peroxymonosulfate. Therefore, the current emphasis of the experimental work is to develop a broad understanding of the chemistry of these species to assess their role in the mechanism of $\mathrm{SO}_{2}$ oxidation. The practical importance of this problem area lies in the probable role of these reactions in causing acid rain, and the possibility of achieving an improved understanding of lime-limestone ilue gas scrubbing.

In this work, pulse radiolysis is lised as a technique to selective?y generate reactive intermediate species of interest. Stopped flow and kinetic spectrophotometry experiments are performed to obtain complementary information on aqueous kinetics. 
(a) For understanding the oxidation of $\mathrm{SO}_{2}$ in solution, the chemical reactions of $\mathrm{SO}_{3}$ must be understood, and much effort was devoted to studying the chemistry of this species during the past year.

The reaction with $\mathrm{O}_{2}$ is of particular interest. Pulse radiolysis experiments established that this reaction has a rate constant of $1.5 \times 10^{9}$ $\mathrm{M}^{-1} \mathrm{~S}^{-1}$, and gives $\mathrm{SO}_{5}^{-}$as a product:

$$
\mathrm{SO}_{3}^{-}+\mathrm{O}_{2} \rightarrow \mathrm{SO}_{5}^{-}
$$

Rate constants for the oxidation of a variety of organic compounds by $\mathrm{SO}_{3}$ have been measured, and equilibrium constants for the reactions of $\mathrm{SO}_{3}$ with chlorpromazine and phenol were also obtained. $\mathrm{SO}_{3}$ was found to be a mild oxidant. The oxidation or several compounds by $\mathrm{SO}_{5}$ was found to occur more rapidly than the corresponding oxidation by $\mathrm{SO}_{3}$.

The reaction of $\mathrm{SO}_{3}$ with the ferrous ion has been investigated by monitoring the product ferric sulfite complex at $350 \mathrm{~nm}$. A product which absorbs at this wavelength initially builds up rapidly $\left(\mathrm{k}-2 \times 10^{7} \mathrm{M}^{-1} \mathrm{~s}^{-1}\right)$ and at longer times continues to build up at a slower rate $\left(\mathrm{k}-2 \times 10^{6} \mathrm{M}^{-1} \mathrm{~s}^{-1}\right)$. These results may be explained by the rapid formation of a ferrous sulfite radical complex followed by a slower electron transfer step.

(b) The oxidation rate of $S(I V)$ by various radicals has been measured. In addition to showing the expected increase in rate with increasing reduction potential of the radical, the ratio of the rates of oxidation of sulfite to that of bisulfite, $\left.\mathrm{k}\left(\mathrm{SO}_{3}^{2-}\right) / \mathrm{kHSO}_{3}^{-}\right)$decreased. For mild oxidants, bisulfite is quite unreactive. This information may help explain some observations on the $\mathrm{pH}$ dependence of $\mathrm{SO}_{2}$ antioxidation in aqueous solutions.

The formation and reaction of sulfite radicals has been investigated more thoroughly by studying the one-electron redox reactions involving sulfite ions and the aromatic amines, aniline, dimethylaniline, diphenylamine, phenylenediamine, and tetrame thyl-phenylendiamine (TMPD). The behavior of the aromatic amines varied from aniline, whose radical cation reacts rapidly with sulfite and slowly with bisulfite to TMPD, which is oxidized by the sulfite radical and whose radical cation reacts with $\mathrm{SO}_{3}^{-}$to form a new species which absorbs at $455 \mathrm{~nm}$.

(c) The kinetics of the reactions of peroxymonosulfite with ferrous and cobaltous ions have been studied using the techniques of stopped flow and kinetic spectrophotometry. The reaction of ferrous ions with peroxymonosulfate is monitored by following the build-up of ferric ion in the stopped flow spectrophotometer. The rate of the reaction, wich has been determined at several acidities and with either ferrous ion or peroxymonosulfate in excess, appears to be a complicated function.

The cobalt-catalyzed decomposition of peroxymonosulfate has aiso been studied. The results show that the Co(III) builds up as the peroxymonosulfate is depleted, suggesting a mechanism such as: 


$$
\begin{aligned}
& \mathrm{Co}^{2+}+\mathrm{HSO}_{5}^{-} \rightarrow \mathrm{CO}^{3+}+\mathrm{OH}^{-}+\mathrm{SO}_{4}^{-} \\
& \mathrm{SO}_{4}^{-}+\mathrm{CO}^{2+} \rightarrow \mathrm{SO}_{4}^{2-}+\mathrm{CO}^{3+} \\
& \mathrm{Co}^{3+}+\mathrm{HSO}_{5}^{-} \rightarrow \mathrm{Co}^{2+}+\mathrm{SO}_{5}^{-}+\mathrm{H}^{+} \\
& 2 \mathrm{SO}_{5}^{-}+2 \mathrm{SO}_{4}^{-}+\mathrm{O}_{2}
\end{aligned}
$$

The study of this slow reaction is complicated by the reduction of Co(III) by water.

(d) The reaction of bisulfite with hydrogen peroxide was examined to determine if the reaction is a radical chain process. In experiments in which ascorbic acid or pyridine were added to scavenge radicals, there was no apparent effect on the measured rate, supporting a non-radical reaction mechanism.

In the future the systematic exploration of the chemistry of $\mathrm{SO}_{3}^{-}$ will be continued with a pulse radiolytic examination of reactions with the ferrous and ferric ions and with other metal ions such as manganese, vanadium, and copper. The reaction of ferric ion with bisulfite will also be studied using an anaerobic mixing system and kinetic spectrophotometry (in collaboration with Dr. Carl Clark of South Carolina State College). In addition, computer modelling will be applied to analyze the experimental data on the kinetics of the reactions of peroxymonosulfite (in collaboration with Dr. N. C. Peterson of Polytechnic Institute of N.Y.).

3. Fluorescence Studies

(C. Lutz, F. P. Schwarz, and P. Ausloos)

Many organic liquids excited by absorption of radiation undergo fluorescence or energy transfer to solute molecules in competition with iragmentation. Measurements of fluorescence quantum yields, energy transfer efficiencies, fluorescence lifetimes, and yields of photofragments in irradiated organic liquids provide information about the nature of the excited states formed by direct excitation, and about the nature of the excited molecules resulting from ion-electron recombination.

In the past year, the following projects were brought to completion:

(a) The study of p-dioxane aggregation with water and with methanol was completed with the determination of the flourescence lifetimes of the emitting aggregates. The aggregate formed in pure dioxane can incorporat,e small amounts of $\mathrm{H}_{2} \mathrm{O}$ or $\mathrm{CH}_{3} \mathrm{OH}$ into its structure, which significantly alters its fluorescence properties. 
(b) The charge transfer from trans-decalin radical cation to a variety of aromatic compounds was monitored through the fluorescence resulting from recombination of the product cation with an electron:

$$
\begin{aligned}
& D^{+}+M \rightarrow M^{+}+D \\
& M^{+}+e \rightarrow M^{*} \\
& M^{*} \rightarrow M+h v
\end{aligned}
$$

It was found that the relative rate constants for charge transfer from the trans-decalin ion to the various solutes increase with increasing exothermicity of the reaction. 


\section{PUBLICATIONS}

(a) Publications of Past Year

Ausloos, P. and Lias, S. G., "Isomerization of Carbonium Ions in Collision Complexes," Int. J. Mass Spectrom. Ion. Proc., 58, 165 (1984).

Baral, S., Hambright, P., and Neta, P., "One- and Two-Electron Reduction of Aluminum and Tin Pyridylporphyrins. A Kinetic Spectrophotometric Study," J. Phys. Chem., 88, 1595 (1984).

Brault, D. and Neta, P., "Reactions of FeIII-Porphyrins with Peroxyl Radicals Derived Pron Haloethane and Halomethanes," J. Phys. Chem., 88, 2857 (1984).

Braun, W., McNesby, J. R., and Scheer, M. D., "A Comparative Rate Method for the Study of Unimolecular Fall-off Behavior, it J. Phys. Chem. 88, 1846 (1984)

Brown, R. L., "Counting of Resonance Structures for Large Benzenoid Polynuclear Hydrocarbons," J. Computational Chem., 4, 556 (1983).

Cvetanovic, R. J., and Singleton, D. L., "Reaction of oxygen Atoms with olefins," Reviews of Chemical Intermediates 5, 183 (1984).

DeMore, W. B., Golden, D. M., Hampson, R. F., Howard, C. J., Kurylo, M. J., Molina, M. J., Ravishankara, A. R., and Watson, R.T., "Chemical Kinetics and Photochemical Data for Use in Stratospheric Modeling," Evaluation No. 6 of the NASA Panel for Data Evaluation, JPL Publication 83-62, Jet Propulsion Laboratory, Pasadena, CA, 1983.

Grodkowski, J. and Neta, P., "One-Electron Oxidation in Irradiated Carbon Tetrachloride Solutions of ZnTPP, TMPD, and Phenols," J. Phys. Chem., 33, 1205 (1984).

Hembright, P., LeMelle, S., Alston, K., Neta, P., Newbali, H. H., and Distefano, S., "A Dissociative Mechanism for the Dithionite Reduction of Cobalt(III)-Myoglobin," Inorg. Chim. Acta, 92, 167 (1984).

Kurylo, M. J. and Knable, G. L., "A Kinetics Investigation of the Gas Phase Reactions of $\mathrm{Cl}\left({ }^{2} \mathrm{P}\right)$ and $\mathrm{OH}\left(\mathrm{x}^{2} \pi\right)$ with $\mathrm{CH}_{3} \mathrm{CN}$ : Atmospheric significance and Evidence for Decreased Reactivity Between Strong Electrophiles," ¿. ?hys. Chem., 88, 3305 (1984).

Laufer, A. H. and Lechleider, R., "The Reaction of Ethynyl Radicals with $\mathrm{O}_{2}$. Rate Constant for Formation of CO," J. Phys. Chem., 88, 66 (1984).

Laufer, A. H., Gardner, E. P. Kwok, T. L. and Yung, Y., "Computations and Estimates of Rata Coeficients for Hydrocarbon Reactions of Interest to the Atmospheres of the Duter Solar Systems," Icarus, 56, 560 (1983).

Lias, S. G., "Ion Thermochemistry - A Summary of the Panel Discussion," Gas Phase Ion Chemistry, Ed. M. A. A. Ferreria, Amsterdam: Reidel, 1984 . 
Lias, S. G. and Buckley, T. J., "Structures and Reactions of $\mathrm{C}_{3} \mathrm{H}_{6}^{+}$Ions Generated in Cyclopropane," Int. J. Mass Spectrom. Ion Proc. 56, 123 (1984).

Martinez, R. I. and Herron, J. T., "Acid Precipitation: The Role of $\mathrm{O}_{3}$-Alkene- $\mathrm{SO}_{2}$ Systems in the Atmospheric Conversion of $\mathrm{SO}_{2}$ to $\mathrm{H}_{2} \mathrm{SO}_{4}$ Aerosol," J. Environ. Sci. Health, A18, 739 (1983).

Martinez, R. I. and Herron, J. T., "Methyl Thi irane: Kinetic Gas-Phase Titration of Sulfur Atoms in $\mathrm{S}_{\mathrm{x}} \mathrm{O}_{\mathrm{y}}$ Systems," Int. J. Chem. Kinetics, 15, 1127 (1983).

Martinez, R. I. and Niki, H., "O-Atom Production via Dissociation of a Dioxymethylene Diradical," Bul1. Soc. Chim. Belges, 92, 657 (1983).

Meot-Ner (Mautner), M., "The Ionic Hydrogen Bond. 4. Intramolecular and Multiple Bonds. Proton Affinities, Hydration and Complexes of Amides and Amine Acid Derivatives," J. Am. Chem. Soc. 106, 278 (1984).

Meot-Ner (Mautner), M., "Structurally Complex Organic Ions: Thermochemistry and Non-Covalent Interactions," Accounts of Chem. Res., 17, 186 (1984).

Meot-Ner (Mautner), M., "The Ionic Hydrogen Bond and Ion Solvation. 1. $-\mathrm{NH}^{+} \cdot \ldots \mathrm{O},-\mathrm{NH}^{+} \cdots \mathrm{N}-$ and $-\mathrm{OH}^{+} \cdots \mathrm{O}-$ Bonds. Correlations iith Proton Affinity. Deviations Due to Structural Effects," J. Am. Chem. Soc. 106, 1257 (1984).

Meot-Ner (Mautner), M., "The Ionic Hydrogen Bond and Ion Solvation 2. Solvation of Onium Ions by 1-7 $\mathrm{H}_{2} \mathrm{O}$ Molecules. Relations Between Monomolecular, Specific and Bulk Hydration," J. Am. Chem. Soc. 106, 1264 (i984).

Morehouse, K. M. and Neta, P., "Redox Reactions of Manganese Porphyrins in Aqueous Solutions. Steady-State and Pulse Radiolysis Spectrometric Studies," J. Phys. Chem., 88, 1575 (1984).

Niki, H., Maker, P. D., Savage, C. M., Breitenbach, L. P., and Martinez, B. I., "Eourier Transform Infrared Study of the Gas-Phase Reaction of $1 \mathrm{O}_{3}$ with trans- $\mathrm{CHCl}=\mathrm{CHCl}$ in ${ }^{16} \mathrm{O}_{2}-\mathrm{Rich}$ Mixtures. Branching Ratio for $0-A$ tom Production via Dissociation of the Primary Criegee Intermediate," J. Phys. Chem., 88, 766 (1984).

Norris, R. K., Barber, S. D., and Neta, P., "Steric Effects on Rates of Daholgenation of Anion Radicals Derived from Substituted Nitrobenzyl Halides," J. Am. Chem. Soc., 106, 3140 (1984).

Scheer, M. D., McNesby, J. R. and Braun, W., "A Study of the Collisional Activation of Cyclobutanone by the Transient Heating of $\mathrm{SiE}_{4}, " \mathrm{~J}$. Phys. Chem. 88, $1850(1984)$.

Schwarz, F. P. and Meot-Ner (Mautner), M., "Eluorescence Quenching of Liquid Alkylbenzenes Excited by Nonionizing and Ionizing Ultraviolet Radiation and by $\beta$ Radiation," J. Phys. Chem. 87, 5206 (1983). 
Shaub, W. and Tsang, W., "Dioxin Formation in Incinerators," Environmental Science and Technology 17, 72 (1983).

Sleck, L. W., "Screening of Aromatic Components in Petroproducts by Chemical Ionization Mass Spectrometry with Chlorobenzene as Reagent Gas," J. Anal. Chem., 55, 38 (1983).

Singleton, D. L., Irwin, R. S., and Cretanovic, R. J., "Mechanism of the Reaction of Oxygen Atoms, $\left(0^{3} \mathrm{P}\right)$ with Dimethyl Disulfide," Can. J. Chem., 61,968 (1983).

Siskos, P. A., Peterson, C. C. and Huie, R. E., "The Kinetics of the Manganese (III) - Sulfur (IV) Reaction in Aqueous Perchloric Acid Solutions," Inorg. Chem., 23, 1134 (1984).

Steln, S. E., Gardiner, W. C., Frenklach, M., and Clary, D. W., "Detailed Kinetic Modelling of Soot Formation in the Shock Tube Pyrolysis of Acetylene," Twentieth Symposium on Combustion, Uni versity of Michigan, Ann Arbor, Michigan, August (1984).

Stein, S. E., Brown, R. L., Griffith, L. and Manka, M., "Chemical Fundamentals of Coal Char Formation," ACS Div. Fuel Chem. Preprints, St. Louis, Missouri, 29(2), 42-8 (1984).

Tsang, W., "Single Pulse Shock Tube Studies on the Decomposition of 1,2Dibromoperfluoroethane and Allyl Bromide," J. Phys. Chem., 882812 (1984).

Walker, J. A., Tsang, W. and Szegvary, L., "Comparative Response of Re-refined and Virgin Lube 0:is to Additives by DSC," Measurements and Standards for Recycled Oil - IV NBS Special Publication 674, 1984, pp. 201.

Wasik, S. P., Schwarz, F. P., Tewari, Y. B., and Miller, M. M., "A Head-Space Method for Measuring Activity Coefficients, Partition Coefficients, and Solubiiities of Hydrocarbons in Saline Solutions," J. Res. NBS 89, 120 (1984). 
Baral, S., Neta, P., and Hambright, P., "Spectrophotometric and Kinetic Studies of the Radiolytic Reduction of Several Pyridylporphyrins and Their Metal Complexes," Radiat. Phys. Chem., (in press).

Baulch, D. L., Cox, R. A., Hampson, R. F., Kerr, J. A., Troe, J. and Watson, R. T:, "Evaluated Kinetic and Photochemical Data for Atmospheric Chemistry: Supplement II," CODATA Task Group on Chemical Kinetics, J. Phys. Ref. Data, (in press).

Brault, D., Neta, P., and Patterson, L. K., "The Liquid Peroxidation Model for Halogenated Hydrocarbon Toxicity. Kinetics of Peroxyl Radical Processes Involving Fatty Acids and Fe(III) Porphyrius," Nature, (submitted).

Brown, R. L., "Rate Constant Uncertainties in Chemical Reaction Models," Computers and Chemistry, (submitted).

Harriman, A. and Neta, P., "Catalyzed Decay of Zinc Porphyrin $\pi$-Radical Cations with Colloidal $\mathrm{RuO}_{2} \cdot 2 \mathrm{H}_{2} \mathrm{O}$ in Aqueous Solutions," J. Phys. Chem., (submitted).

Huie, R. E. and Neta, P., "Oxidation of Ascorbate and a Tocopherol Analogue by the Slifite Derived Radicals $\mathrm{SO}_{3}{ }^{-}$and $\mathrm{SO}_{5}{ }^{-}$," Environ. Res., (in press).

Huie, R. E. and Neta, P. "The Chemical Behavior of $\mathrm{SO}_{3}{ }^{-}$and $\mathrm{SO}_{5}{ }^{-}$Radicals in Aqueous Solutions," J. Phys. Chem., (in press).

Huie, R. E. and Neta, P., "Reaction of the Canadate Ion With ChIoropramazine and Chlorpromazine Free Radical With the Vanadly Ion," Inorg. Chem. Acta., (in press).

Laufer, A. H., "Vinylidene $\left({ }^{3} B_{2}\right)$ - An Active Intermediate in the Photolysis of Ethylene," J. Photochemistry, (in press).

Lias, S. G., Liebman, J. F., and Levin, R. D., "Evaluated Gas Phase Basicities and Proton Afifinities of Molecules; Heat of Formation of Protonated Molecules," J. Phys. Chem. Ref. Data, (in press).

Lias, S. G., "Thermoneutral Isotope Exchange Reactions in Proton-Bound Conplexes of Water with Organic Molecules: Correlations with Energetics of Formation of the Corresponding Association Ions," J. Phys. Chem., (in press).

Lias, S. G., Jackson, J. A., Argentar, H., and Liebman, J. F., "Substituted N,N-Dialkyl Arilines: Relative Ionization Energies and Proton Afiinities through Determination of Ion-Molecule Reaction Equilibrium Constants," J. Org. Chem., (submitted).

Lias, S. G. and Ausloos, P., "Structures of $\mathrm{C}_{6} \mathrm{H}_{7}{ }^{+}$Ions Formed in Unimolecular and Bimolecular Reactions," J. Chem. Phys., (submitted).

Manka, M. J. and Stein, S. E., "Disproportionation-Recombination Rate Ratios for Hydroaromatic Radicals," J. Phys. Chem., (in press). 
Martinez, R. I., "The Photooxidation of Formaldehyde: A Different Perspective," Int. J. Chem. Kinetics, (submitted).

Meot-Ner (Mautner), M., and Deakyne, C., "Unconventional Ionic Hydrogen Bonds: $\mathrm{CH}^{\delta+} \ldots \mathrm{X}$ and $\mathrm{NH}^{+} \cdots \pi$ Interactions. Complexes of Quarternary Ions with Solvents and of Onium Ions with $\pi$-Donors", J. Am. Chem. Soc. (submitted).

Meot-Ner (Mautner), M., Nelsen, S. F., Willi, M. F., Frigo, T. B., "Special Effects of an Unusually Large Neutral to Radical Cation Geometry Change: Adiabatic Ionization Energies and Proton Affinities of Alkylhydrazines," J. Am. Chem. Soc., (submitted).

Meot-Ner (Mautner), M. and Sieck, L. W., "The Ionic Hydrogen Bond and Ion Solvation. 3. $\mathrm{SH}^{+} \cdots \mathrm{O}$ and $\mathrm{NH}^{+} \cdots \mathrm{S}$ Bonds. Correlations with Proton Affinity. Mutual Effects of Weak and Strong Ligands in Mixed Clusters," J. Phys. Chem. (submitted).

Morehouse, K. M. and Neta, P., "Kinetics of Demetallation of Manganese (II) Porphyrins in Aqueous Solutions," J. Phys. Chem., (in press).

Neta, P. and Simic, M. G., "Free Radical Chemistry of Natural Products," Proceedings of Institut of Food Technologists Basic Symposium, (in press).

Neta, P. and Harriman, A., "Zinc Porphyrin $\pi$-Radical Cations in Aqueous Solution. Formation, Spectra and Decay Kinetics," J. Phys. Chem., (submitted).

Scheer, M. R., Braun, W., and McNesby, J. R., "The Application of a New High Temperature Reactor to Unimolecular Decompositions," Chem. Phys. Lett., (submitted).

Scheer, M. D., "A Kinetic Isotope Effect in the Thermal Dehydration of Cellobiose," Proceedings of the International Conference on Iundamentals of Thermal Biomass Conversion, (in press).

Schwarz, F. P. and Smith, D., "The Yields of Excited States of Aromatic Derivatives in the Radiolysis of Hydrocarbon Solutions," Radiat. Phys. and Chem., (in press).

Schwarz, F. P., "Positive Charge Transfer in the UV Photolysis and B Radiolysis of trans-Decalin Solutions," J. Phys. Chem., (submitted).

Schwarz, F. P., Okabe, H., and Ausloos, P., "Fluorescence Studies of the Plise RadioIysis of the Halogenated Derivatives of Methane and Ethane," $J$. Phys. Chem., (submitted).

Shaud, W. M. and Tsang, W., "Overview of Dioxin Eormation In Gas and Eolid Phases Under Municipal Incinerator Conditions," ACS Symposium Series Special Publication, (in press).

Shaub, W. M. and Tsang, W., "Hazardous iaste Aspects of Municipal Solid 'Naste Incineration," Proceedings of Conferences on Hazarcous Naste Management, Pergamon Press (in press). 
Sieck, L. W. and Meot-Ner (Mautner), M., "Thermal Decomposition of Ions;

1. Pyrolysis of Protonated Ethers-Activation Energies and A Factors," J. Phys. Chem., (in press).

Sieck, L. W. and Meot-Ner (Mautner), M., "Thermal Decomposition of Ions; 2. Pyrolysis of Protonated t-Butylmethylether at the Low Pressure Limit. Activation Efficiencies for Various Bath Gases," J. Phys. Chem., (in press).

Stein, S. E. and Brown, R. L., "A Chemical Theory of Graphite," Carbon, (in press).

Stein, S. E., Brown, R. L., and Greenberg, A., "Relative Thermochemical Stabilities and Reactivities of Benzo[A]pyrene and Selected Isomers, "The Chemistry of the Total Environment, (in press).

Stein, S. E., "Free Radicals in Coal Conversion," Coal Conversion Chemistry, R. Schlosberg, Ed., Pergamon Press, 1984, (in press).

Tsang, W., Macek, A., Domalski, E., Walker, J. A., Charagundl, S. R., Colbert, J. C., Kirklin, D. R., Ledford, A. E., Deiker, P. H. and Ryan, R. V., "Combustion Characteristics of Refuse Derived Fuels," NBSIR, (in press).

Tsang, W. and Shaub, W. M., "Tracers for Quality Assurance in Municipal Solid Waste Incineration," NBSIR, (submitted).

Tsans, W. and Hampson, R. F., "Chemical Kinetic Data Base for Methane Cambustion," J. Phys. Chem. Ref. Data, (submitted).

Tsang, W., "Single Pulse Shock Tube Study on The Thermal Stability of Ketones," Int. J. Chem. Kinet., (in press).

Tsang, W., "Hazardous Waste Aspects of MSW Combustion," Proceedings of Energy from Municipal Waste Workshop, NBSIR, (in press).

Tsang, W. and Walker, J. A., "Chemical Processes During the Starved Air Incineration of Municipal Solid Waste," NBSIR, (submitted).

Tsang, W., "Stability of Alkyl Radicals," J. Am. Chem. Soc., (submitted).

Walker, J. A. and Tsang, W., "RDF Characterization Using Thermal Methods of Analysis," NBSIR, (submitted).

Westley, F., "Tables of Experimental Rate Constants for Chemical Reactions Occurring in Combustion (1978-1982). Part I. Reactions of Inorganic Compounds with other Species. Part II. Reactions of Organic Compounds with Other Species," (submitted). 


\section{CHEMICAL THERMODYNAMICS DIVISION \\ Eugene S. Domalski, Acting Chief}

SUMMARY OF ACTIVITIES

Fiscal Year 1984

1. INTRODUCTION

A. Objectives

The Center Thermodynamics Division is a discipline-oriented branch of the Center for Chemical Physics and is concerned primarily with the quantitative relationships that govern the transformation of energy from one chemical system or form to another. The Division seeks to establish itself as a leader in this field through the certification of calorimetric standards, performance of precise thermochemical measurements, and evaluation and compilation of thermodynamic data. Particular emphasis is directed toward the implementation of a research program in biothermodynamics which offers fundamental data for industrial applications to the production of chemicals, food, and fuels by means of biocatalytic processes.

Specific objectives of the Chemical Thermodynamics Division are:

to advance the science of calorimetry through the design of novel calorimeters, development of improved procedures, and attainment of enhanced measurement precisions and accuracies.

- to develop and certify standard reference materials for calorimetric measurements.

- to carry out calcrimetric and related measurements from which thermodynamic properties can be derived on key chemical substances or processes.

- to provide compilations on the chemical thermodynamic properties of inorganic and organic conpounds as well as miscellaneous materials from evaluation of the data reported in the literature.

- to develop estimation methods for the prediction of chemical thermodynamic properties of organic and inorganic compounds in the saseous, liquid, solid, and aqueous phases.

- to respond to the needs of industry, academia, and government for thermodynamic, and related information. 
(b) To determine the equilibrium constants for the isomerization reactions mentioned in (a) as a function of temperature using HPLC methods.

(c) To determine the enthalpies of the enzymatic elimination of ammonia by aspartic acid, phenylalanine, and tyrosine as a function of temperature using microcalorimetry.

(d) To determine the equilibrium constants for the enzymatic elimination of ammonia using HPLC or GC methods.

Deliverables

(a) Thermodynamic properties ( $\Delta H, \Delta S, \Delta G, K$, percent conversion) as a function of temperature.

(b) Generalizations regarding: common molecular substrate sites vulnerable to enzymatic conversion, separation of enthalpy and entropy effects.

2. Energetics of Nucleic Acid Bases and Nucleosides

\section{Rationale}

Thermodynamic properties must be available on key nucleic acid moieties so that an understanding of the intra- and intermolecular bonding energetics can be derived. The availability of a sufficient set of thermodynamic properties will allow for the development of estimation schemes so that the energetics of other related heterocyclic nitrogen compounds can be predicted without the need for experimental measurements.

Experimental Approach

(a) To determine the enthalpies of combustion and solution of crystalline adenine, purine, guanine, adenosine, and guanosine using combustion bomb calorimetry and solution calorimetry.

(b) To develop an estimation procedure which accommodates the available enthalpy of formation $\left(\Delta_{\mathrm{r}} \mathrm{H}\right)$ data on nucleic acid bases and nucleosides and can be used to predict the $\Delta_{\mathrm{r}} \mathrm{H}^{\prime} \mathrm{s}$ for other related heterocyclic nitrogen compounds. The development of estimation procedures is oriented toward ultimately providing the biochemist/bioengineer with a predictive capability for thermodynamic properties in the aqueous phase.

Deliverables

(a) Expansion of the predictive capability for the enthalpy of formation to nucleic acid bases and nucleosides. 
The Chemical Thermodynamics Division is organized into two major activities: thermodynamic measurements and thermodynamic data compilation. These activities are under the supervision of two group leaders, Eugene S. Domalski, Acting Division Chief, and David Garvin, Director of the Chemlcal Thermodynamics Data Center, respectively. The Division has 15 full time staff, 2 guest workers, 3 part time employees, 4 contractors, 1 consultant, 2 rehired annuitants, 1 research associate, and 1 summer student. Three persons have been reassigned to other Divisions within NBS, which leaves the Division with an overall staff of 32 persons.

\section{Biothermodynamics Research Program}

A research program in biothermodynamics has been developed in FY 1984 which constitutes a major part of the overall NBS effort in biotechnology. The biothermodynamics research program should provide the measurements and data which will be useful to the chemical industry as it introduces bio-processes into the production of chemicals, foods, and fuels. Certain research areas were easily implementable in the program for the Division while others required longer periods to initiate because of the need for the purchase of an instrument, reassignment of manpower, or establishment of a research agreement with a private vendor.

The biothermodynamics research program consists of the following tasks:

1. Thermodynamics of Enzyme-Catalyzed Reactions

Rationale:

Studies carried out on enzyme-catalyzed reactions have shown that enzymes vary in the cegree of their substrate specificity. Some enzymes are very specific while others can tolerate significant changes in the substrate with little change in the attainment of chemical equilibrium or in the general nature of the reaction product formed. The formation and breaking of bonds, as a result of an enzyme interacting with a substrate, centers around specific sites in the substrate molecule.

As a result of examining a general reaction tyoe ( $\mathrm{NH}_{3}$ elimination) catalyzed by several related enzymes and several reactions (isomerization) caused by the same enzyme, we hope to provide fundamental information regarding the specific products, the energetics, and equilibria of these reactions.

Experimental Approach

(a) To determine the entralpies of reaction for the isomerization of glucose to fructose, xylose to xylulose, and ribose to ribulose as a function of temperature using glucose isomerase by microcalorimetry. 
4. Measurements and Standards on Biochemical Systems using DSC

\section{Rationale}

For many years differential scanning calorimetry (DSC) has been used to study conformational energies and phase changes in proteins, lipids, and biopolymers. Such transitions provide useful characterizational information which often show relationships between thermal phenomena and molecular structure. Thermal interactions between enzymes and various substrates can be measured and should yield information regarding the nature of the chemical binding in such systems. The need for temperature and enthalpy of transition standards in research on biochemical substances in aqueous solution demands additional focus on DSC measurements for selected systems.

\section{Approach}

(a) To determine whether interactions between glucose isomerase and various monosaccharide substrates can be shown to have a stabilizing effect on the enzyme-substrate complex.

(b) To determine whether the DSC thermogram can serve as standard characterizational tool which may identify enzyme stability, enzyme deactivation (denaturation), or specific enzyme transitions.

(c) To assess whether phase transitions in selected lipids (such as DPPC) or proteins can serve as standards for temperature of transition or enthalpy of transition for biochemical systems in aqueous solution.

\section{Deliverables}

(a) Characterization of enzyme-substrate interactions as well as a clearer understanding of enzyme stabilization.

(b) Development of temperature and enthalpy of transition standards for biochemical systems in aqueous solution.

5. Compilation of Tables of Thermodynamic Properties of Important Biochemical Substances

Rationale

Tables of critically evaluated thermodynamics properties of biomolecules in aqueous solution are needed by biologists, biochemists, and biochemical engineers involved in making thermodynamic assessments as calculating the energetics of chemical reactions or establishing the equilibria for a collection of reacting species. A variety of compilations have been published (although none recentIy) but have been omnibus in nature and not specifically oriented toward the biochemical or biotechnological community. 
(b) Extension of thermodynamic data on nucleic acid bases and nucleosides by combining available $\Delta_{\rho} H$ data, $\Delta H$ solution, solubilities, entropy, $\Delta H$ fusion, $\Delta H$ vaporization.

\section{Weak Interactions in Model Enzyme-Substrate Systems}

\section{Rationale}

Research is in progress in which the solubilities of nonelectrolytes are being measured in aqueous solution as a function of concentration and temperature using the generator column technique. These measurements will allow for the derivation of values indicative of the weak interactions responsible for such phenomena as enzyme stabilization, enzyme-substrate binding, enzyme immobilization, base stacking and intercalation in polynucleotides. Systematic measurement of a key set of values for weak inter-functional group interactions should provide the foundation for a predictive capability useful in explaining some aspects of the phenomena mentioned above in aqueous solution.

\section{Experimental Approach}

(a) To determine the solubilities of non-electrolytes in aqueous solution as a function of concentration and temperature using the automated coupled-column chronatographic apparatus.

(b) To focus on interactions between aromatic hydrocarbons and organic compounds containing $-\mathrm{OH},-\mathrm{CH}_{3}$, and $-\mathrm{CONH}_{2}$ functional groups.

Deliverables

(a) Development of an estimation methodology based on the additivity principle will be derived from the set of values for interactions obtained experimentally and will allow for the prediction of weak interactions between small molecules containing these groups.

(b) As data accumulates from the measurements and $l$ arger molecules are examined, computer modeling with functionalgroup interaction potentials can be used. From the estimation methodology based on larger molecules, prediction of model interactions of phenomena such as enzymesubstrate binding, enzyme stabiiization, and protein folding will be possible. 


\section{Rationale}

Gibbs energy maps are of importance in determining product yields for biochemical reactions, for the meaningful comparison of thermodynamic measurements made on biochemical solutions under a wide variety of experimental conditions, and for the calculation of the equilibrium properties of solutions.

\section{Approach}

R. A. Alberty (J. Biol. Chem. 243, 1337 (1968); ibid. 244, 3290 (1969)) in a series of classic papers described the construction of Gibbs energy maps and their application to biochemical systems. A shortcoming in his treatment was that he did not include activity coefficients in his model, 1.e., he assumed ideal behavior of the solute species. Since Alberty's paper was published in 1969, there have been very significant advances in computation and in numerical analysis software which makes the inclusion of activity coefficients and other improvements possible. In addition to obtaining amounts of species in solution, it is also possible to calculate activity and osmotic coefficients and the thermodynamic excess properties of aqueous solutions. A paper describing this type of generalized procedure is now in preparation and it has also been applied to a publication on the treatment of the thermodynamics of the $\mathrm{SO}_{2}$ $+\mathrm{H}_{2} \mathrm{O}$ system.

\section{Deliverables}

(a) R. N. Goldberg, "On the Use of an Equilibrium Model for the Calculation of the Activity and Osmotic Coefficients in Aqueous Solutions," J. Res. Nat. Bur. Stand., 89,251 $(1984)$.

(b) R. N. Goldberg and V. B. Parker, "Thermodynamics in Solution of $\mathrm{SO}_{2}(g)$ in Water and Aqueous Sulfur Dioxide Solutions," in review.

The fundamental Idea which is used is that all of the equilibrium properties of a solution can be calculated from a knowledge of the equilibria in solution and assumption(s) concerning the non-ideal behavior of the soiute species in the solution.

At present we are able to handle equilibrium calculaticns involving up to about ten different species in solution (including trace amounts) as well as being able to calculate the excess thermodynamic properties. These calculations can be performed on multi-component as well as binary solutions. Generic types of interactions which are accounted for include ion-pairing, complexation, hydrolysis, and hydration. The temperature range over which calculations have been performed is 0 to $150^{\circ} \mathrm{C}$. 


\section{Approach}

(a) To prepare a table of critically evaluated thermodynamic data $\left(\Delta H, \Delta G, S, C_{D}, K\right.$ at $298 K$ ) on amino acids, peptides, and heterocyclic compounds.

\section{Deliverables}

Availability of critically-evaluated thermodynamic data on amino acids, peptides, and heterocyclic compounds from a common up-to-date reference source.

6. Estimation of Thermodynamic Properties of Important Biochemical Substances

\section{Rationale}

The usefulness of compilations on critically evaluated thermodynamic data of organic and bio-organic compounds can be maximized when such compilations become the foundations for predictive or correlational schemes. The availability of evaluated data on $\Delta_{f} H, S$, and $C_{p}$ for organic and bio-organic compounds in the condensed and aqueous phases offers a unique opportunity to develop estimation and correlational schemes for such compounds. As mentioned before, the ultimate goal is to provide the biochemist/bioengineer with a predictive capability for thermodynamic properties in the aqueous phase.

Approach

(a) To develop Benson-type group cortribution values for the estimation of the enthalpy of formation, entropy, and heat capacity at $298 \mathrm{~K}$ in the condensed phase and in aqueous solution.

(b) To use the Benson approach to develop an estimation method for heterocyclic nitrogen compounds in the gaseous, liquid, solid, and aqueous phases.

Deliverables

(a) Estaclishment of a predictive capability for organic and bio-organic compounds at $298 \mathrm{~K}$ in the condensed and aqueous phases.

(b) Expansion of the CHETAH Program (developed by ASTM Subcommittee $E-27.07$ on Estimation Methods) for the prediction of thermodynamic properties $\left(\Delta_{f} H, S, C_{p}\right)$ of organic compounds in the condensed and aqueous phases with a focus on bio-organic compounds. 
8. Revision of Thermodynamic Data for Waste Incineration

\section{Rationale}

A report (NBSIR 79-1479) lists thermal properties (primarily specific heat capacity and gross heat of combustion) of over 300 waste components including a large number of biomass species. An up-to-date edition of this report is underway as part of a SERI-NBS Research Agreement to expand the listing of waste materials to between 500-600. Particular attention has been will be placed upon expansion of the available data on biomass materials. New data on other waste components will also be included.

\section{Approach}

(a) Past research and evaluation efforts on thermodynamic data compilation will be used. About 100 to 150 materials have been evaluated previously.

(b) The recent literature will be searched for available thermodynamic data on waste components. Special emphas is will be placed on the collection and evaluation of biomass materials.

\section{Deliverables}

(a) An up-dated compilation containing thermodynamic data on waste components and biomass materials, entitled: "Thermodynamic Data for Biomass and Waste Incineration."

9. Thermodynamics of the Krebs Cycle and Related Reactions

\section{Rationale}

The Krebs or citric acid cycle is the major metabolic pathway in aerobic organisms for the oxidation of sugars, fatty acids, and some amino acids. Many of its reactions and substrates are involved in biosinthetic pathways for carbohydrates, lipids, and proteins. It is the central mechanism for metabolic processes in almost all celis. A fundamental understanding of the thermodynamic equilibria of these processes has applicability toward the optimization of industrially-related bioconversion reactions for current and future needs.

$\underline{\text { Approach }}$

(a) Thermodynamic data of the species invoived in the Krebs cycle reactions are being evaiuated.

(b) A least sums - least squares solution to the network of reactions wilch comprise the Krebs cycie processes are being studied. Emphasis is on the Giobs energies of reactions in aqueous solution. 


\section{Deliverables}

(a) Evaluated thermodynamic properties for the Krebs cycle reactants and products in aqueous solution at $25^{\circ} \mathrm{C}\left(\Delta_{f} \mathrm{H}\right.$, $\left.\Delta_{\mathrm{f}} G, S, \Delta_{\mathrm{f}} S, K\right)$.

(b) A least sums - least squares solution to the network of reactions which comprise the network of the Krebs cycle reactions.

D. Standards for Calorimetry

Proposals for research in the development of standards for calorimetry were submitted to the office of Standard Reference Materials in May 1984 and two projects were awarded funds for work in FY 1985. Both were continuations of on-going projects in the following areas: (1) development of a standard reference material (SRM) for the combustion calorimetry of organic phosphorus compounds and (2) continuous testing of SRM coal samples for chemical stability and integrity on standing or in storage. The latter is monitored through determinations for moisture, ash, and calorific value of the SRM coals.

Our calorimetric standards activity on an SRM for organic phosphorus compounds is complemented by the collaborative research program which has begun with the U.S. Department of the Army, Aberdeen, MD to carry out calorimetric mesurements and develop estimation methods for organic phosphorus compounds. 
E. Other Agency Research

Other agency research accounts for about 25 percent of the Division's resources and consists of both experimental and data programs. Some have been renewals or add-on programs to those already in place while others are new research efforts. The Division's other agency research programs are summarized below:

\section{Funding Agency}

Department of Defense Air Force office of Scientific Research (AFOSR)

\section{Department of Energy \\ Pittsburgh Energy \\ Technology Center (PETC)}

Department of Energy Energy from Municipal iaste (EMW)

Design Institute for Physical Property Data (DIPPR)

U.S. Department of the Army, Aberdeen Proving Ground

Solar Energy Research Institute (SERI)

\section{NBS Program Manager/} Investigator

S. Abramowitz

D. A. Ditmars

D. Garvin

R. N. Goldberg

V. B. Parker

D. Smith-Magowan

E. S. Domalski

K. L. Churney

A. E. Ledf ord

S. S. Bruce

T. J. Buckley

D. Garvin

R. N. Goldberg

T. L. Jobe, jr.

E. S. Domalski

D. R. KirkIin

E. S Dcmalski

I. L. Jobe, Jr.
Program Title/ Description

High temperature thermodynamic measurements on refractory substances, pure elements and compounds

Thermodynamic data for flue gas clean-up in cossil fuel technology

Chlorine content of municipal solid waste; Threshold condition for the formation/desiruction of chlorinated organic species.

Bibliographies of thermodynamic data on sour water and various salt systems.

Combustion calorimetry and estimation methods for organic phosphorus compounds.

Revision and up-date of previous publication on thermodynamic data for waste incineration. 
A. Thermodynamics of Enzyme-Catalyzed Reactions

(E. Gajewski, R. N. Goldberz, D. Steckler, and Y. B. Tewari)

Accurate thermodynamic data on biochemical reactions are needed to facilitate a better understanding of the reactions themselves and for the optimization of product yields for reactions which have industrial significance. The goal of this effort is to provide reliable themodynamic data (equilibrium constants, enthalpies, and heat capacities) for biochemically and industrially important reactions. The accomplishments this past year are in several areas: (1) measurements on specific enzyme-catalyzed reactions, (2) expansion of laboratory capability and method development, and (3) computational procedures (see section on Modelling of Complex Aquecus Solutions).

Specific enzyme-catalyzed reactions which have been investigated are: (1) The conversion of glucose to eructose, (2) xylose to xylulose, (3) pumarate to malate, and (4) aspartate to fumarate and ammonia. A summary of the results of these investigations follows.

$\operatorname{Process}(A): D$-zlucose $(a q) \longrightarrow D$-Pructose $(a q)$, in the presence of glucose isomerase.

Using a combination of high-pressure liquid chromatography (HPLC) and microcalorimetry, the following values, which refer to a reference temperature of $298.15 \mathrm{~K}$, were determined for process $(\mathrm{A}): \Delta G^{0}=345 \pm 53 \mathrm{~J}$ mol ${ }^{-1}, \Delta H^{\circ}=2780=200 \mathrm{Jmol}^{-1}$, and $\Delta C_{j}^{0}=75=30 \mathrm{Jmol}^{-1} \mathrm{~K}^{-1}$. The HPLC measurements yielded values of $K_{A}$ ercm 298 to $358 \mathrm{~K}$. The values of $K_{A}$ were found to be independent of the reaction being carried out eitner in phosphate or TRIS/HCQ buffer and also independent of the use of either solubilized or immobilized enzymes. Microcalorimetric measurements yielded vaiues of $\Delta H_{\AA}^{\circ}$ and $\Delta C_{D}^{\circ}, A$ determined from the temperature derivatives of the equilibrium constants which were in good agreement with the values of $\Delta r_{\AA}$ obtained from the microcalorizetric investigation. The results of this inyestization are of particular interest since iarze quantities ( $=2 \times 10^{6} \mathrm{~kg} / \mathrm{year}$ ) of fructose-rich corn syrup are being prodliced each year using this reaction. Using the above thermocynamic parameters, the maximum product yielc for this process was calculated erom 298 to $373 \mathrm{~K}$.

Process $(B): \quad x y l o s e(a q) \longrightarrow x y l u l o s e(a q)$, in the presence of giuccse isomerase.

The above reaction is an intermediate step in the conversion of hemicelulose, a major constituent of biomass, to ethanol; this process may have significant industrial applications. The approach adopted in ihis invesigation was ine same as was used in the glucose to eructose stlidy, 
namely a combination of HPLC and microcalorimetry. To date, HPLC measurements have been periormed from 298 to $343 \mathrm{~K}$ and microcalorimetric measurements from 313 to $332 \mathrm{~K}$. Tentative values for process (B) at a reference temperature of $298.15 \mathrm{~K}$ are $\Delta G^{\circ}=4,380 \mathrm{~J} \mathrm{~mol}^{-1}, \Delta \mathrm{H}^{\circ}=16,300 \mathrm{~J} \mathrm{~mol}^{-i}$, and $\Delta \mathrm{C}_{\mathrm{p}}^{\circ}=39 \mathrm{~J} \mathrm{~mol}^{-1} \mathrm{~K}^{-1}$.

Process $(C):$ fumarate $(\mathrm{aq}) \longrightarrow$ malate $(\mathrm{aq})$, in the presence of fumarase enzyme.

This is a fundamental biochemical reaction which occurs in the Kreb's cycle. In the food industry, L-malic acid is used as an acidulent in fruit and vegetable juices, carbonated soft drinks, jams, and other food products; in the pharmaceutical industry, L-malate salts of basic amino acids are used in amino acid infusions. One of the aims of this investigation was to test a quantitative derivatization/gas chromatographic (GC) procedure (see below) which was introduced in this laboratory for the measurement of equilibrium constants for process (C) from 298 to $320 \mathrm{~K}$. Microcalorimetry was used to determine values of $\Delta H_{C}^{\circ}$ from 298 to $320 \mathrm{~K}$. Tentative values for process (C) at $298.15 \mathrm{~K}$ are: $\Delta G_{\mathrm{C}}^{\circ}=-3,540 \mathrm{~J} \mathrm{~mol}^{-1}$, $\Delta \mathrm{H}_{\mathrm{C}}^{\circ}=-15,700 \mathrm{~J} \mathrm{~mol}^{-1}$, and $\Delta \mathrm{C}_{\mathrm{P}}^{\circ}=41 \mathrm{~J} \mathrm{~mol}^{-1} \mathrm{~K}^{-1}$. The value of $\Delta \mathrm{G}_{\mathrm{C}}^{\circ}$ at $298.15 \mathrm{~K}$ corresponds to a value of $\mathrm{K}_{\mathrm{C}}=4.17$ and is in agreement with the value of $\mathrm{K}_{\mathrm{C}}=4.22$ determined by Bock and Alberty ( $\mathrm{J}$. Am. Chem. Soc. 75, 1921 (1953)) using a spectrophotometric procedure. The effects of variations of the ionic strength were examined in both the GC and in the microcalorimetric measurements.

Process $(D):$ aspartate $(\mathrm{aq}) \longrightarrow$ fumarate $(\mathrm{aq})+\mathrm{NH}_{4}{ }^{+}$, in the presence of aspartase enzyme.

The literature contains several values for $K_{D}$ which range from 0.008 to $0.04 \mathrm{~mol} \mathrm{Kg}{ }^{-1}$. The measurement of $K_{D}$ and of $\Delta H \AA$ is complicated by the fact that samples of aspartase are generally contaminated with fumarase which catalyzes process $(C)$. We are currently investigating several techniques which may serve to eliminate or to significantly lessen this problem. These techniques include: (1) carrying out the reaction at a temperature where fumarase is inactive, (2) introducing a chemical inhibitor of fumarase into the reaction mixture, and (3) separating the aspartase from the fumarase using electrophoresis and/or HPLC.

The microcalorimetry laboratory has been modernized this past year. Features which have been introduced include the completion and installation of a third microcalorimeter, installation of skid-state temperatures controllers which utilize proportional and reset modes of operation, and the interfacing of all those microcalorimeters to a microcomputer. This latter feature permits us to operate all three microcalorimeters simultaneously and to perform a reasonable number of measurements ( 9 to 15) in one day. All of the calculations are also performed on the microcomputer.

The measurements of the equilibrium constants of the conversion of

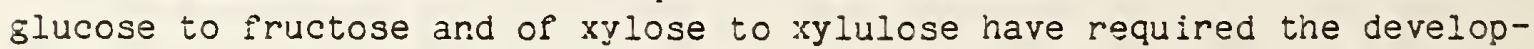
ment of the proper separation of the reactants and products from each 
other and fror the buffer, enzyme, and cofactors. This time consuming and difficult procedure should be significantly aided by the recent installation of a modern HPLC in this laboratory.

An alternative to HPLC and to spectrophotometry is lyophilization. This is a procedure in which a reaction mixture is rapidly frozen in liquid nitrogen, lyophilized or freeze-dried, derivatized (e.g., with N,O-bis(trimethylsilyl)trifluoroacetanide or BSTFA) and then analyzed in a capillary-column gas chromatograph. This type of methodology is of very general application to many biochemical reactions and has been demonstrated to work satisfactorily for the measurement of the equilibrium constant for the conversion of fumarate to malate. We plan to use it for the determination of the equilibrium constant for the conversion of fumarate to aspartate and other related enzyme-catalyzed systems.

Our immediate plans include the completion of the xylose/xylulose equilibrium measurements. The development of finding a method to eliminate the fumarase catalyzed side-reaction from the aspartate/fumarate equilibrium is in progress and will facilitate the measurement of the equilibrium constants and enthalpy changes for process (D) above as a function of temperature. A study of the conversion of ribose to ribulose is also planned as well as an investigation of the conversion of glucose 6-phosphate (G6P) to fructose 6-phosphate (F6P). This investigation would serve to connect the following reaction equilibria in the thermodynamic cycle: $(H)=(F)+(G)-(E)$.

$$
\begin{aligned}
& \text { Glucose }+A T P=G 6 P+A D P \\
& \text { Fructose }+A T P=F 6 P+A D P \\
& \text { Glucose = Eructose } \\
& G 6 P=F 6 P
\end{aligned}
$$

A study of the conversion of glucose to glucose 6-phosphate will follow. This study will complete the thermochemical cycle: $(\Xi)=(I)+$ $(\mathrm{J})$.

$$
\begin{aligned}
& \text { Clucose }+\mathrm{H}_{3} \mathrm{PO}_{4}=\mathrm{G} 6 \mathrm{P}+\mathrm{H}_{2} \mathrm{O} \\
& \mathrm{ATP}+\mathrm{H}_{2} \mathrm{O}=\mathrm{ADP}+\mathrm{H}_{3} \mathrm{PO}_{4}
\end{aligned}
$$

We pian aiso to study transaminase catalyzed reactions of the type:

$$
\mathrm{NH}_{3}+\mathrm{RCOO}=\mathrm{RCH}\left(\mathrm{NH}_{2}\right) \mathrm{COO}^{-}
$$

This generic type of reaction is relevant to the synthesis of amino acids, such as phenylalanine and tyrosine. In addition, we plan to use venicillin acylose to carrying out the reaction: 
(or benzyl penicillin) Penicillin $G \rightarrow 6$-amino-penicillanic acid $(L)$ (6-APA)

+ phenyl acetic acid

The above reaction is a direct route to the synthesis of 6-APA which in turn is used for the synthesis of many different kinds of antibiotics.

B. Weak Interactions in Model Enzyme-Substrate Systems

(S. P. Wasik, H. DeVoe, R. H. Wood, M. M. Miller, and J. Owens)

Intra- and intermolecular interactions between functional groups and other moieties of molecules dissolved in an aqueous medium play crucial roles in a variety of properties and reactions. For example, the tertiary structure of a slobular protein determined by non-bonded intramolecular interactions, as well as by hydrogen bonding. The binding of a substrate molecule to an enzyme also depends on these interactions, the objective of this research is to measure the free energy, enthalpy and entropy components of the interaction of several hydrophobic solutes with a variety of molecules dissolved in water, and to analyze these quantities in terms of either group-group interactions or solute dimerization. These data are needed for understanding the physical basis of hydrophobic effects, for predicting various solubility and binding properties, and for refining empirical potential functions for the pairwise interaction of molecular groups immersed in water. The information is of great interest to tecinnologies which deal with stability and binding effects involving macromolecules, such as enzyme-substrate specificity, to researchers in the area of molecular recognition and host/guest chemistry when hydrophobic groups are involved, and to those who calculate aqueous properties by Monte Carlo and molecular dynamies computations.

The proposed research, already begun, is based upon measurements of the soluoility of a solid hydrophobic compound in aqueous solutions containing a second solute (the additive). At a given temperature and pressure, the chemical potential of the dissolved hydrophobic solute in the saturated solution is constant regardless of the concentration of the additive. Therefore, the effect on the solubility of changing the additive concentration can be interpreted as an activity coefficient effect; or, alternatively, as a free energy of interaction between the hydrophobic molecule and the additive species. The data (solubility versus molality of additive) from measurements with additives containing different proportions of the same functional groups is treated by the method of R. H. Wood to provide the free energies of group-group interactions. The temperature dependence of these free energy parameters yields the enthalpy and entropy components of the interactions.

The aqueous solubilities are measured using a coupled-column Iiquid chromatography (CCLC) method developed at NBS. A generator column is packed with crystals of the solute, or with a chromatographic solid support coated with the solute. Pure water or an aqueous solution of the additive is pumped through the column to saturate the solution with the solute. The total solute concentration in the aqueous phase is found by 
extracting the solute from a known volume onto an extractor column, and eluting this solute onto a reverse phase analytical column for determination by HPLC.

An automated version of the CCLC apparatus was used to measure the aqueous solubilities of five n-alkylbenzenes (ethylbenzene through $\mathrm{n}$-hexylbenzene) over the temperature range $7-45^{\circ} \mathrm{C}$. The automated system Includes control by a microcomputer of temperature, valves, the HPLC pump, and the integrator. From the data, values of $\Delta \mathrm{G}^{\circ}, \Delta \mathrm{H}^{\circ}$, and $\Delta \mathrm{S}^{\circ}$ at $25^{\circ} \mathrm{C}$ for the process solute(liquid) = solute(aqueous) were calculated using the statistical model of Clarke and Glew [Trans. Faraday Soc. 62, 539 (1966)]. The measured values were compared with literature values, where available, and agreement was excellent. This work is being prepared for publication.

The automated CCLC apparatus has been modified to allow it to change the aqueous solutions pumped through the generator column for studies of solute-additive interactions. The solubility of naphthalene, pyrene, and other hydrophobic solutes will be determined in aqueous solutions of a number of alcohols, amines, and heterocyclic species buffered when necessary to prevent ionization. The studies will be made over a range of additive concentration and at three or more temperatures. The results will be analyzed in terms of free energy, enthalpy, and entropy contributions to group-group interactions.

Hydrophobicity scales for the side chains of the naturally occurring amino acids will be constructed from these contributions according to the Savage-Wood additivity principle [J. Solution Chem. 5, 733 (1976)]. This principle assumes that the total interaction between two solute molecules is the sum of all possible group-group interactions. One such scale, for instance, would be for pyrene and would characterize the interaction (hydrophobic bonding) of pyrene with different amino acids in the aqueous environment. This scale, because it is based on actual hydrophobic interactions, would be an improvement over existing hydrophobicity scales which were calculated from data for the transfer of amino acid side chains from water to the vapor phase or a nonaqueous solvent.

A second study will be undertaken to search for measurable interactions of benzene with polycyclic aromatic hydrocarbons in water. Tucker and Christian [J. Phys. Chem. 83, 426 (1979)] have observed activity coefficient effects in aqueous solutions of benzene which they interpret as benzene dimerization. It would be of great interest for the understanding of hydrophobic effects to determine the thermodynamics of the formation of heterodimers of benzene + pyrene, for instance.

During FY 1984, Michele M. Miller transfered from the Chemical Thermodynamics Division to the Organic Analytical Research Divisior. 
C. Bomb Calorimetry of Key Biochemical Compounds

(D. R. Kirklin and J. C. Colbert)

Precision bomb calorimetry has been used to measure the enthalpies of combustion of thermodynamically important molecules to derive precise values for their enthalpies of formation. There are many biochemical molecules which play an active role in biologically important processes for which the enthalpy of formation has not been determined. It is necessary to have precise values for enthalpies of formation to understand the stability or reactivity of such molecules in important bio-processes.

\section{Enthalpy of Combustion of Anhydrous Guanosine}

The enthalpy of combustion of an anhydrous sample of guanosine was measured in the NBS aneroid adiabatic bcmb calorimeter. Guanosine is a nucleoside of one of the two purine bases found in DNA and RNA. Purine, adenine, adenosine, guanine and the dihydrate of guanosine were studied in previous years in this bomb calorimeter. The calorimetric data of anhydrous guanosine was correlated with that of guanine and also that of the adenine/adenosine base/nucleoside pair.

Considerable sample preparation was necessary to obtain meaningful calorimetric data on anhydrous guanosine. Anhydrous guanosine readily absorbs moisture from the atmosphere to form the dihydrate. The internal combustion bomb atmosphere must be saturated with water vapor during a combustion experiment. Therefore, the commercial dihydrate of guanosine was dehydrated in a vacuum oven and encapsulated in paraf in wax before each calorimetric experiment. The "waxed" anhydrous guanosine samples were exposed to a water saturated bomb atmosphere of $3 \mathrm{MPa}$ for 24 hours to determine whether or not the anhydrous samples would remain anhydrous during the equilibration period of the calorimetric experiment. The results proved that the anhydrous samples can be successfully encapsulated in paraffin wax.

The energy of combustion of the paraffin wax utilized was measured to determine the energy correction for its combustion in the calorimetric experiments of "waxed" anhydrous guanosine. The measured energy of combustion for paraffin wax was $-46689.21 \pm 5.84 \mathrm{~J} \cdot \mathrm{g}^{-1}$.

The enthalpy of formation of anhydrous guanosine was calculated from the measured energy of combustion and then correlated with that of our previous measurements on purine bases and their corresponding nucleosides. The enthalpies of formation of glianosine and guanine $\left(-950.10 \mathrm{~kJ} \cdot \mathrm{mol}^{-1}\right.$ and $-183.89 \mathrm{~kJ} \cdot \mathrm{mol}^{-1}$ ) were correlated with that of acenosine and adenine $\left(-651.58 \mathrm{~kJ} \cdot \mathrm{mol}^{-1}\right.$ and $\left.+96.92 \mathrm{~kJ} \cdot \mathrm{mol}^{-1}\right)$. The mean difference between a nucleoside and 1 ts corresponding purine base was calculated to be -757.36 $\mathrm{kJ} \cdot \mathrm{mol}^{-1}$. This mean difference allows the calculation of the enthalpies of formation for any purine nucleoside from a measured or literature value of that of the purine base with an accuracy of the order of 1 percent. 
Combustion bomb calorimetric measurements were completed on a sample of D-ribose in FY 1983. The molar enthalpy of combustion at $303.15 \mathrm{~K}$ for seven measurements is $\Delta_{\mathrm{C}} \mathrm{H}=-2349.26 \pm 0.68 \mathrm{~kJ} \cdot \mathrm{mol}^{-1}(2 \mathrm{sdm})$. Studies were carried out in FY 1984 to characterize the D-ribose sample using DSC and NMP. methods. DSC measurements did not show sharp melting for the sample and estimates of purity from such tests were calculated to be -97 percent. NMR studies suggest the presence of four anomeric forms in the solid phase, two being major and two extremely small. From an examination of the literature, we suspect that the most predominant form which comprises - 65 percent of the solid phase is the 8 -pyranose form and that the next most prevalent for which amounts to - 35 percent is the $\alpha$-pyranose anomer. The $\alpha$ - and $\beta$ - furanose anomers probably constitute the remainder, however, additional NMR studies are planned for EY 1985.

\section{DSC Measurements and Standards}

Efforts in fiscal year 1984 were directed toward expanding our instrumental capabilities in differential scanning calorimetry (DSC). The Division has had a Perkin-Elmer DSC-II system which is useful for studying the heat capacities, melting points, and phase transitions of pure solid substances. The need to study biochemical systems in aqueous solution required procurement of a DSC instrument which has (1) a larger cell volume (1 to $2 \mathrm{ml}$ ) than the Perkin-Elmer DSC-II (0.06 to $0.10 \mathrm{ml})$, (2) capability for scanning in both a heating and cooling mode, (3) versatility with sample cell removal and cleaning, (4) availability with a suitable data acquisition system and software package, (5) temperature scan rates as $10 \mathrm{w}$ as $1^{\circ} \mathrm{C} /$ hour, and (6) scan rate reproducibility of -0.01 percent. After examining the commercially available instruments, the Hart Scientific Model 7707 DSC was selected. Delivery is expected in September 1984.

Plans for research in FY 1985 will follow three areas of interest: (1) determination of the suitability of lipids as standard materials for identifying temperature and enthalpies of transition for biochemical systems in aqueous solution, (2) establishing the thermal character of enzymes, (i.e., denaturation, complexation, reversible transitions), and (3) determination of the thermal character of glucose iscmerase through interactions with monosaccharide substrates and co-factors in aqqueous solution.

Personnel assignments to the DSC studies will include two full-time scientists and a zuest worker on a part-time basis.

E. Calorimetric Standards/OSRM

(D. R. Kirkiin and J.C. Colbert)

The NBS effort in precision bomb calorimetry permits the certification of primary and secondary calorimetric standards that are used worldwide to calibrate and check calorimetric procedures. The primary calorimetric standard is benzoic acid which has been available iron NBS 
since the 1920 's. Secondary calorimetric standards are needed for compounds which contain elements other than carbon, hydrogen and oxygen and therefore require slightly modified calorimetric procedures to obtain precise and accurate data.

Three projects were performed to determine secondary calorimetric standards. Sulfur is an important element in many organic compounds, and therefore, a secondary combustion standard is necessary for sulfur-containing compounds. In consumer oriented areas, fuel laboratories need secondary calorimetric standards for the types of fuels which are being utilized. The OSRM distributes SRM coals which are certified for trace elements and sulfur content. Their certification has been extended to include a certified calorific value. Since the long term ( $>1$ year) stability of coal samples is not known, these SRM coal samples are recertified annually. In addition, the development of a refuse-derived fuel (RDF) as an article of commerce requires a calorimetric standard which is free of variability due to geographical location and ambient conditions. Refuse-derived fuels are primarily cellulose with varying amounts of non-combustible materials and water. Therefore, a synthetic RDF was developed with fixed cellulose and non-combustible components for certification as a standard reference material (SRM).

\section{Certification of the Enthalpy of Combustion of Thianthrene}

Primary and secondary standards are necessary to calibrate calcrimeters and to check calorimetric procedures. The primary standard, benzoic acid, is useful for the calibration of oxyzen bomb calorimeters but cannot be used to check the procedures necessary for organic sulfur compounds. A secondary standard containing sulfur is needed. $N$. H. Johnson (a former NBS employee) examined thianthrene (diphenyl disulfide) and found it suitable for a secondary sulfur standard. The office of Standard Reference Materials (OSRM) contracted with the Aldrich Chemical Company, Inc. of Milwaukee, WI to prepare a thianthrene sample of suitable purity to be used as a SRM. Calorimetric measurements were performed on the Aldrich thianthrene sample for certification as a secondary conbustion calorimetry standard.

The NBS aneroid adiabatic calorimeter was used to make measurements of the enthalpy of combustion because a combustion product of organic sulfur compounds is sulfur dioxide. Inside the sealed combustion bomb the sulfur dioxide is oxidized to sulfur trioxide which reacts with the water in the bomb to form aqueous sulfuric acid. To adequately account for the energy produced from the formation of sulfuric acid instead of sulfur dioxide, the sulfuric acid must be made homogeneous by rotation of the bomb after the combustion is complete before the equilibration afterperiod. Hence, the unique NBS rotating adiabatic bomb calorimeter was necessary for this project. Three sets of measurements were nade using different amounts of water in the bonb for the formation of sulfuric acid. It was found that the minimum amount of water required is three times the volume of the bomb in cubic decimeters. 
The results of the bomb calorimetric measurements yielded a certified value of $33511.56 \mathrm{~J} \cdot \mathrm{g}^{-1}$ with a standard deviation of the mean of 2.39 $\mathrm{J} \cdot \mathrm{g}^{-1}$ when the sample is burned under certificate conditions using a quantity of water in the bomb equal to or greater than three times the volume of the bomb in $\mathrm{dm}^{3}$.

\section{Certification of a Synthetic Refuse-Derived Fuel}

A synthetic refuse-derived fuel (RDF) was prepared from 80 percent microcrystalline cellulose, 15 percent silica, and 5 percent alumina. The mixture was blended and stored in 13 SRM gross containers. During the container filling process, samples were taken from the bottom and top of each container to determine the homogeneity of the blended material. The material was tested for ash content and residual moisture.

The samples were randomly placed in seven sets of six analysis samples. Six analysis samples per set were chosen because of the geometry of our moisture oven. This allowed duplicate measurements on all but six samples. Robert Paule, a NML statistician, analyzed the results to determine the sample variability. The sample variability was found to be less than 1.3 percent for moisture (which is affected by the ambient relative humidity) and less than 0.35 percent for dry ash. The sample was judged to be suitable for a standard reference material (SRM) at these levels of variability.

The gross samples were bottled with a sample size suitable for issuance as NBS SRM 1657. A similar protocol has been devised to determine the certified caloriflc value. Moisture and ash will be redetermined to verify that no sample segregation has occurred during the bottling process. These measurements are currently underway.

\section{Coal Combustion Calorimetry Standards}

The heats of combustion of SRM 1632a (a bituminous coai) and SRM 1635 (a sub-bituminous coal) are being measured annually so that they can be used as secondary combustion calorimetry standards. Secondary combustion calorimetry standards are necessary to check calorimetric and analytical procedures on materials that have combustion characteristics similar to those materials encountered in laboratory analyses. Since fossil fuels are routinely encountered in commercial laboratories there was a great need for certified heats of comoustion of coal samples. However, since coal samples are oxidized slightly upon storage under laboratory conditions, the certified heats of combustion are remeasured. SRM coal specimens are taken from a sample bottle that is being stored under laboratory conditions and calorimetric measurements are performed annually to check the certified heats of combustion.

SRM coals 2682, 2683, 2684, and 2685 which were certified for suliur content, calorific value and ash content during the previous fiscal year have been included in this variability study. The SRM sample bottles that were used in the orizinal certification are also being stored under 
laboratory conditions. Specimens are taken from the sample bottles and determinations are made annually for moisture, ash content, and calorific value.

F. Large Sample Combustion Flow Calorimetry

(E.S. Domalski, D. R. Kirklin, J. C. Colbert, K. L. Churney, M. L. Reilly, A. E. Ledford, Jr., S. S. Bruce, and T. J. Buckley.)

Financial support for this activity has come from the U.S. Department of Energy, Energy from Municipal Waste Division, Office of Renewable Technology.

\section{The Calorific Value Problen}

Incinerator-boiler systems which are used to dispose of municipal solid waste (MSW) are bought and sold on the basis of thermal specifications. To determine whether a system meets its thermal specifications, the calorific value of the input waste stream must be known. At present, the calorific value is determined at commercial test laboratories by a bomb calorimetric procedure. This procedure involves using gram-size samples derived from MSW that has been processed down to a particle size of $2 \mathrm{~mm}$ or less. Critics of the procedure argue that gram-size samples are too small to be representative of such a large quantity of so heterogeneous a material, and that processing MSW may also alter its composition. The latter might occur because of excess local heating in some of the milling operations or because of sampling and handling of a material which is inhomogeneous and segregates easily.

The general question of whether or not the calorific value of a multi-ton quantity of MSW can be obtained by sampling to one or two grams can be broken into two parts. The first part is: how should one sample a multi-ton pile or stream of raw MSW to obtain increments of suficient number and size to characterize the calorific value with a given precision? The second part is: how can one obtain representative gram-size samples that characterize these measurements? The dispute about the validity for determining the calorific value of MSW is primarily concerned with the second of these questions.

To test the bomb calorimetric procedure, a combustion flow calorimeter that can accommodate a $2.5 \mathrm{~kg}$ sample of MSW was designed and constructed during 1981 through early 1983. This size of sample was selected as a convenient function of a sampling increment for the waste stream on the basis of extensive conversations with combustion engineers and statisticians.

During FY 1983, experimental calorimetric work was initiated to address two problems. The first was the sampling problem: how does one obtain a representative gram-size sample from a kilogram-size quantity of highly processed MSW (i.e., < $2 \mathrm{~mm}$ particle size)? The second was the processing problem: Does processing MSW down to a $2 \mathrm{~mm}$ particle size alter its calorific value significantly (i.e., one percent or more)? 
During the course of this study 22 flow calorimetric measurements using $2.5 \mathrm{kilogram-size} \mathrm{samples} \mathrm{and} 16$ bomb calorimetric measurements using 2.5 gram-size samples were carried out. In FY 1984, results were calculated, analyzed, and presented in four talks and two publications. The results in brief are as follows:

The sampling problem: Calorimetric data on processed MSW were obtained using both the kilogram-size flow and a gram-size bomb calorimeter. The sampling division (i.e., $2.5 \mathrm{~kg}$ to $2 \mathrm{~g}$ ) was done with a Brinkmann riffler because the method is insensitive to the effects of segregation. The calorific values (on a dry basis) of the gram-size test samples and other kilogram-size parents agree within the uncertainty of our experiments. The average difference of the parent minus gram-size test samples was -0.1 percent with an imprecision (95 percent confidence level) of 1.1 percent of the mean calorific values.

The processing problem: The effects of processing on sample composition were determined by intercomparison of processed and minimally processed MSW ( $150 \mathrm{~mm}$ or less particle size) that were nominally identical. Reduction of the particle size of the sample was carried out in a Wiley Mill. Powdered dry ice was added to the sample to prevent excessive local heating. The difference of the unprocessed calorific values on a dry basis was -0.5 percent with an imprecision of 2.9 percent of the mean calorific value. Most of the imprecision is due to the sample preparation procedure; it did not yield identical processed and unprocessed samples.

These results show that representative gram-size test samples can be obtained from $2.5 \mathrm{~kg}$ increments provided excessive local heating is avoided in reducing the sample particle size and the sample division technique is insensitive to segregation. Our overall results are that the difference in the calorific value (on a dry basis) of the unprocessed parent $2.5 \mathrm{~kg}$ sample minus the gram size processed test sample is -0.6 percent with an imprecision of 3.1 percent of the mean calorific value.

During FY 1985, we plan to perform combustion calorinetric studies on refuse samples under excess-air, stoichiometric-air, and starved-air conditions. No data are available for validating metinods for measurement of the degree of incomplete ccmbustion and the calculation of corrections to calorific values obtained with test samples in 100 percent oxyzen as compared to air-combustion conditions in an incinerator. This work is needed to reduce the gap between data from laboratory tests and operation of real-world combustion systems.

\section{Organo-chicrine Compounds Prom MSir Comoustion}

Widespread reports of the presence of dioxins and related compounds in emissions Irom municipal waste combustors have proven to be a major impediment in the use of MSW as a fuel in energy recovery units. The reported dioxin levels vary greatly between combustion facilities. Since there seem to be no intrinsic thermodynamic or kinetic $(e .8$. , at least in the gas phase) barriers to dioxin destruction, modifications in operation or design of a facility should lead to minimization of emissions of 
dioxins. The empirical data-base needed for developing a strategy for this minimization (e.g., studies related dioxin levels to incinerator operating variables) is just beginning to appear. Unfortunately, the growth of this data-base is hindered by high costs and long time scales for the necessary research to be conducted.

Two problems are being studied at NBS. The first problem is to determine the quantity and distribution of chlorine in municipal solid waste. Very little data exists (one study of the chlorine content on five days of sampling MSW in Wayne County, Michigan, in 1979). The second problem is to determine the threshold conditions for the formation and/or destruction of organo-chlorine compounds in the combustion of MSW. This problem is being studied in conjunction with the threshold conditions for the formation of other products of incomplete combustion (e.g., CO, soot-carbon, various hydrocarbons, sulfur containing molecules) to see if these last mentioned species, which are easier and cheaper to monitor, can serve as indicators of dioxin formation and/or destruction. The threshold problem is closely related to work on the calorific value determinations to be performed in $\mathrm{O}_{2} / \mathrm{N}_{2}$ mixtures in FY 1985.

Sampling of MSW was carried out at two sites, each for periods of five days. Five days samples were obtained from Baltimore County, Maryland, and $f i v e$ days samples from Brooklyn, New York to provide some idea of differences between MSW from different areas. About 13-20 kg of refuse was sampled daily from the output of the primary shredder of the Baltimore County Resource Recovery Facility in Cockeysville, Maryland. The New York samples were obtained by removing five to seven increments of MSW on each of Pive days from the receiving pit of the Green Point Incinerator in Brooklyn, New York. All samples were dried and hand sorted into combustible components (paper, hard and soft plastics, textiles, wood/vegetable matter, and $f$ ines/sweepings) and non-combustible components (glass, metals). Each of the combustible components has been processed to < $2 \mathrm{~mm}$ particle size, blended (i.e., homogenized), and sampled for analysis to determine water-soluble chlorine and total chlorine content. A reconstituted sample of each of the ten days waste was prepared to determine the chlorine and the total sulfur content of the original (i.e., unsorted) MSW. Analytical determinations are now camplete and being evaluated.

\section{Combustion Studies to Establish Threshold Conditions}

The 2.5 kilogram capacity flow calorimeter will be used to pursue combustion measurements to establish the threshold conditions for the formation/destruction of chlorinated species. Although the conbustion studies will be carried out in the calorimeter, no heat measurements will be made to calculate a calorific value since this datum is not applicable to the proposed study. Threshold conditions will be established from the measurements of the following parameters:

Variation in the MSW composition, including synthetic and real-world RDF samples doped with chiorine compounds. 
- Variation of the input flow rate $\left(\mathrm{O}_{2}\right.$ concentration) under excess-air, stoichiometric-air, and starved-air conditions.

- Temperature profile of the reaction zone and post-reaction zone of the combustor.

- Determination of the chlorine content of the MSW stream, particulates ( $f$ ly ash when formed), bottom ash, and gaseous effluent.

The detection of chlorine-containing species in the gaseous effluent will be carried out using IR and GC-MS methods. Information regarding the limits of on-line detection of gaseous chlorine species will be derived from these studies. The results of the combustion studies will link the threshold conditions for the formation/destruction of chlorinecontaining species to the degree of decomposition of the MSW sample. The influence of cool-zones in the combustor will be investigated to find out what effect their presence has on the kind and quantity of chlorinated species which can be found in the gaseous effluent and ash residue.

Trial combustions in air have begun. Analytical characterization of organo-chlorine compounds is being carried out by the Organic Analytical Research Division of NBS.

G. Drop Calorimetry of Non-Reacting Systems Below $2800 \mathrm{~K}$ (D. A. Ditmars)

Financial support for this work has come from the U.S. Department of Defense, Air Force Office of Scientific Research.

This activity represents a continuation of a calorimetric research program whose goals include:

1. to obtain accurate thermodynamic data on non-reacting refractory materials in either the solid or liquid state up to $2800 \mathrm{~K}$.

2. to measure specific heat at temperatures above $273 \mathrm{~K}$ of wellcharacterized materials of the highest attainable purity for which existing $C_{p}$ data are insufficient or in conflict and which are: 1) critical to energy- and space-related technology, or 2) especially suited as models on which to test refined lattice-dynamic calculation of thermodynamic properties.

3. to produce and support high-temperature calorimetric standard reference materials (SRM's) for heat capacity and for differential thermal analysis (DTA) calorimetric measurements.

The number of facilities, nationally, which can sustain programs of high-temperature calorimetric research is seriously diminishing at a time of increasing needs for just the data produced through this type of investigation. There is a constant demand for thermodynamic data on new materials capable of functioning acceptably in the harsh environments 
associated with space propulsion systems or upon re-entry, new energy technologies and defense-generated needs for materials to enable space systems to withstand overt aggression. Also, the existing thermodynamic data on some elements, for which it is now possible due to theoretical advances to calculate directly some thermodynamic functions, is of ten not accurate enough to allow a meaningful test of theory.

The Chemical Thermodynamics Division has available two operative precision drop calorimeters for the ranges 273-1200 K (isothermal, phase-change calorimeter) and 1200-2800 K (adiabatic receiving calorimeter). These are being used in research intended to provide calorimetric measurements at the highest accuracy on materials related to the hightemperature applications mentioned above. Every effort is made to coordinate this research with that of another NBS facility (very-hightemperature pulse calorimeter) in order to provide the capacity for continuous coverage of high-temperature thermodynamic studies up to $5000 \mathrm{~K}$ or above on especially important systems. We have in addition an operative high-temperature discrete-heating adiabatic calorimeter $(300-800 \mathrm{~K})$ and we are installing a low-temperature $(4-300 \mathrm{~K})$ adiabatic calorimeter for future research needs.

In order that NBS thermal data and that of other high-temperature investigations can be fairly intercompared and assessed for accuracy, we maintain a continuing effort to update or extend the range of coverage of high-temperature standard reference materials. In recognition of the growing need for accurate DTA temperature and enthalpy standards we attempt to identify systems whose thermal properties can be measured by both conventional calorimetric and DTA techniques.

Fiscal year 1984 was devoted to completion of heat-capacity measurements on $\alpha$-and $B-\mathrm{Si}_{3} \mathrm{~N}_{4}$ in the range $273-1200 \mathrm{~K}$ and on hexagonal ("graphitic") BN and cubic ("diamond-like") BN in the same temperature range. Both sets of data for the two BN polymorphs are consistent with available low-temperature data and conform more reasonably to anticipated behavior of these substances at hign temperatures than the literature data would indicate.

These materials ( $\mathrm{Si}_{3} \mathrm{~N}_{4}$ and $\mathrm{EN}$ ) are widely used in the fabrication of components for service at high temperatures in chemically aggressive environments and especially where high resistance to mechanical and thermal shock are important. Accurate high-temperature thermodynamic functions for $\mathrm{Si}_{3} \mathrm{~N}_{4}$ and $\mathrm{BN}$ (each with two polymorphs) should provide useful engineering data and assist in theoretical interpretations of the $\mathrm{Si}-\mathrm{N}$ and $\mathrm{B}-\mathrm{N}$ systems.

In addition in FY 1984, we measured the heat capacity of fcc (face centered cubic) calcium of high chemical purity in the range 273-716 $\mathrm{K}$. Calcium is a CODATA "key substance". Accurate knowledge of the thermodynamic properties of this element is essential to a correct thermochemical description of a large number of technologically important calcium compounds. The thermodynamic data on calcium up to $200 \mathrm{~K}$ rests on a tenuous base. Above $200 \mathrm{~K}$, to the fcc-bcc transition temperature $(716 \mathrm{~K})$, 
rellable data was entirely lacking. The heat capacity from 200 to $716 \mathrm{~K}$ was formerly calculated by judicious extrapolation and constraint of the extrapolation to behave in "an anticipated fashion" relative to similar $\mathrm{Mg}$ and $\mathrm{Sr}$ data. Our measurements in the range 273-716 $\mathrm{K}$ have yielded Improved thermodynamic functions for calcium (with a caveat related to the $C_{p}$ data below $200 \mathrm{~K}$ ) and will place future CODATA evaluation of $\mathrm{Ca}$ compounds on a more secure footing.

In fiscal year 1985, we will extend the heat capacity measurements on one or more of the nitride polymorphs described above to temperatures above $1200 \mathrm{~K}$. Highest service temperatures are not yet well-defined for these materials. We plan as well to investigate the fcc-bcc transition of calcium at $716 \mathrm{~K}$ and perhaps begin measurements on the bcc (body centered cubic) phase. If guest-worker participation can be realized, we will initiate heat capacity studies on very pure copper with the view in mind of establishing copper as a substitute heat-capacity standard and of providing the most accurate heat-capacity data available for comparing to theoretically calculated values for $C_{p}$.

H. Raman Characterization of the Main Phase Transition in Lipid Bilayers (W. H. Kirchhoff, Research performed at the National Institutes of Heal th)

Within the past ten years, Raman spectroscopy has been lised with increasing popularity to characterize the main phase transition (gel to liquid crystal) in lipid bilayers. In spite of the growing use of this technique, no attempt has yet been made to investigate quantitatively the shapes of intensity-ratio versus temperature profiles and to interpret those shapes in terms of the thermodynamic properties of the phase transition.

A simple, two-state model in which the distribution of domain sizes does not vary as a function of temperature has been deveioped for expressing the intensity ratio of Raman spectral features as a function of temperature. The model provides the following analytical expression for the ratio of intensities at two scattering frequencies:

$$
R_{12}=I\left(v_{1}\right) / I\left(v_{2}\right)=\frac{A+\alpha\left(T-T_{0}\right)}{1+e^{t n}}+\frac{B+B\left(T-T_{0}\right)}{1+e^{-t n}}
$$

where $T_{0}$ is the midpoint of the phase transition and is defined as the cemperature at wich the chemical potentiais of the iwo states are equally shifted by a smali but determinable amount due to the mathematics of monitoring intensity ratios rather than the intensities themselves; $t$ is a reduced temperature $=\left(T-T_{0}\right) / D$ and where $D$ is nelated to the enthalpy difference between states $A$ and $B$ by $D=R T / \Delta \bar{H} A B$ and $B$ are functions of the intensities of the states $A$ and $B$ at the ${ }^{\circ}{ }^{\circ}$; ${ }^{\circ}$ different scattering frequencies $\nu_{1}$ and $v_{2}$ and $\alpha$ and 3 are empirical slopes in the asymptotes of the sigmoidal profile introcuced to account for domains of small size; and $n$ is an effective number of molecules per domain. Depending on the 
density of data points in the phase transition interval, $n$, as determined by a least squares fit of the analytical expression to the Raman intensity ratios, varies between the root mean square of the domain size to

$\left\langle v^{2}\right\rangle /\langle n\rangle$. For broad domain size distributions, $\left\langle n^{2}\right\rangle /\langle n\rangle$ can be an order of magnitude greater than $\langle n\rangle$.

From the least squares fit of the analytical expression to the spectroscopic data, the simple model reproduces the temperature profiles to within experimental error. Improvements in the accuracy of intensity measurements, in the precision of temperature control, and in the accuracy of temperature measurement, all of which are achievable with existing instrumentation, should allow for examination of somewhat more complex models.

At present, the computer program which has been written allows for a systematic analysis of Raman spectroscopic temperature profiles and provides an independent estimate of van't Hoff enthalpies of phase transitions. This work has been performed in cooperation with scientists at the National Institutes of Health and the computer program which has been developed is now used for the routine analysis of Raman spectroscopic temperature profiles.

\section{Future Plans}

In biothermodynamics, on-going programs will continue beyond FY 1986 with the likelihood of expansion such as: biomass conversion to saccharides, measurement of activities and activity coefficients of dilute and concentrated sugar solutions, and measurements of redox potentials, biochemical couples, and low temperature heat capacity measurement on key compounds of interest in industrial biochemistry. Research in combustion bomb calorimetry will pursue measurements on oligopeptides in order to test the constancy of the energy of the peptide bond. Similar measurements are applicable to oligosaccharides in which the constancy of the energy of the oxygen or ether linkage between repeating saccharide units can be examined.

The rationale for pursuing some of the new research tasks in biothermodynamics in FY 1985 and beyond is given below:

1. Heat Capacities and Absolute Entropies of Biomolecules and Biopolymers

An understanding of the conformational changes which occur in biomolecules and biopolymers as a function of temperature provides important thermal data needed to explain the behavior of proteins, lipids, and carbohydrates in industrial biochemical proceses. Absolute entropies are needed along wiht enthalpy data to calculate equilibrium properties over temperature intervals of interest. Heat capacities provide information regarding the nature of intramolecular transitions (e.g., ist order vs. 2nd order). 
Entropy data are derived from accurate measurement of the heat capacity over a temperature range extending from the liquid helium range eup to room temperature. The specific technique applicable to these measurements, low temperature adiabatic calorimetry, has been demonstrated in the literature to be also a useful technique for identifying conformational changes of biomolecules and for probing the interaction of biopolymers with are molecules and ions of dissolved salts.

\section{Biomass Conversion to Saccharides}

Conversion of biomass (cellulosic materials) to saccharides, such as glucose, can be accomplished by either acid hydrolysis or enzymatic hydrolysis. Several advantages are possible with the enzymatic approach because it allows for lower operating costs and does not require corrosion-resistant equipment. High temperatures are required in acid hydrolysis of cellulosic materials because of the need to break down crystalline structure. However, high temperatures cause decomposition of the resulting surgars. Enzymes can convert cellulosic materials to various oligosaccharides at moderate temperatures, have high specificity, and do not react with other non-cellulosic materials or impurities.

We plan to study the enzymatic conversion of cellulose to glucose and hemicellulose to xylose as initial systems. Pretreatment methodologies will be examined after simple cellulosic materials have been measurd for their ability to undergo saccharification. Later, attention would be given to the bioconversion of lignocellulosic materials.

\section{Measurements of Activities and Activity Coefficients}

The activity of biomolecules in solution and an understanding of their departure from ideal solution behavior are of importance from both a theoretical and practical standpoint. Occassionally, equilibrium constants disguise or cancel the impact of non-ideality in solution processes. Interpretation of equilibrium data in which several reaction products are formed requires a knowledge of activities and activity coefficients to understand the observed yielcs.

\section{Redox Potentials and Biochemical Couples}

There are several important biochemical processes which "couple" with other biochemical processes to bring about chemical reactions which otherwise would not occur. For example, the phosphorylation of gluocose to glucose-6-phosphate is not thermodyanmically favorable:

$$
\text { Glucose }+\mathrm{H}_{3} \mathrm{PO}_{4}=\text { Glucose-6-phosphate }
$$

However, when adenosine 5'-triphosphate (ATP) is present, the following reaction is favorable:

$$
\text { Glucose } \div A T P=\text { Glucose-6-phosphate + ADP }
$$


The difference between the two processes above is the hydrolysis of ATP to adenosine $5^{\prime}$-diphosphate (ADP):

$$
\mathrm{ATP}+\mathrm{H}_{2} \mathrm{O}=\mathrm{ADP}+\text { phosphate }
$$

The above process couples not only with glucose but also with other carbohydrates and with several hundred other substrates. Other important biochemical couples and redox potentials involve nictotinamide adenine dinucleotides ( $N A D^{+} / \mathrm{NADH}$ ), nicotinamide adenine dinucleotide phosphate $\left(\mathrm{NA}-\mathrm{DP}^{+} / \mathrm{NADPH}\right)$, and Plavin adenine FAD (FAD/FADH 2 ). Because of the ubiquitous nature of these redox potentials and couples in biochemical reactions, a precise understanding of the their thermodynamics is of both fundamental and practical importance.

The recertification of the energy of combustion of benzoic acid will take place during FY 1986 - FY 1990 and should be accompanied by efforts to improve the degree of precision and accuracy by one order of magnitude. Similar efforts toward improving the precision and accuracy of the enthalpy of combustion of methane will be considered. These improvements in the precision and accuracy of combustion calorimetric measurements are partly due to recent advances in the development of large sample capacity combustion bomb and flow calorimetry.

We anticipate that the U.S. Department of the Army will support research in the Division on the combustion calorimetry and estimation procedures for organic phosphorus compounds. The development of standard state corrections to calorimetric data for organic phosphorus compounds should evolve naturally from this work.

In large sample combustion flow calorimetry, we expect that collaborative research between NBS and Warren Spring Laboratory in the U.K. will be carried out in which a comparison of combustion results on samples of municipal solid waste wlll be made in kilogram-capacity calorimeters.

High temperature heat capacity using drop calorimetry will be pursued for calcium and strontium metals from their melting points to room temperatures. These measurements will assist theoretical calculations on the lattice dynamics of bcc and fcc systems. In addition, high temperature measurements will be performed on high purity zirconia from $1200 \mathrm{~K}$ to room temperature. 


\section{THERMODYNAMIC DATA COMPILATIONS}

\section{A. Chemical Thermodynamics Data Center}

The Chemical Thermodynamics Date Center has as its mission the preparation and distribution of reliable thermochemical data suitable for use throughout science and industry. The work is supported by the office of Standard Reference Data (OSRD), the CCP biotechnology program, the Department of Energy (DOE), and the Design Institute for Physical Property Research (DIPPR). Closely related work is carried on in the Electrolyte Data Center and the Ion Energetics Data Center.

These recommended data are prepared by reassessment, selection and synthesis from published experimental measurements and theoretical calculations, and, where necessary, prediction of values. The principal types of data contained in our data bases of selected values are enthalpies of formation, Gibbs energies of formation, entropies, enthalpy increments and heat capacities. The major classes of input data are enthalpies of combustion, reaction, solution and phase change; equilibria in reactions, emf cells, solubility and vaporization; heat capacities and enthalpy changes and molecule parameters irom spectral data. Gases, liquids, solids, solutions, and ions (gaseous and in solution) are considered.

The applications of the recommended thermodynamic data are in the calculation of heat balances in industrial processes, the calculation of equilibrium yields in chemical reactions, calibration and cesting of experimental equipment, correlation and prediction of properties of closely related compounds and interpretation of measurements. The activities during the past year are reported here under three headings: Evaluation and Numerical Data Bases; Accessions, Information and Support; and Autcmation Activities.

1. Evaluation and Numerical Data Bases

(W. H. Evans, D. Garvin, V. B. Parker, R. L. Nuttal1, R. H. Schumn, and D. D. Wagman)

In biothermodynamic data efforts, work has been performed in two areas: (1) the compilation of tables of thermodynamic properties of important biological compounds, and (2) the estimation of thermodynamic properties by group contribution value techniques.

A search of a major organic data-base provided to the Chemical Thermodynamics Data Center by J. B. Pedley, University of Sussex, was carried out to extract and evaluate the enthalpies of formation at $298 \mathrm{~K}$ of amino acids, peptides, and heterocyclic compounds. Additions to this collection on enthalpies of formation data were made from the recent iterature.

A major compilative effort of the Chemical Thermodynamics Data Center has appeared in the literature during isscal year 1984 and is entitied" Heat Capacities and Entropies of Organic Compounds in the Conaensed Phase" 
by E. S. Domalski, W. H. Evans, and E. D. Hearing, (J. Phys. \& Chem. Ref. Data 13, supplement no. 1, 1-286 (1984)). Data on the heat capacities and entropies of over 1400 organic compounds in the liquid and solid phases are provided. Values for the enthalpies and entropies of phase transitions (solid/solid, fusion, vaporization, and sublimation) which were encountered as part of the evaluation are included in the tables. Over 800 references were examined and cover the period from 1881 to 1982. The search of the literature, data evaluation, and computerized-tabulation of the data and bibliography were carried out primarily through contracts with local vendors.

Group contribution values are being generated for use in the estimation of the following properties at $298 \mathrm{~K}$ on organic compounds: enthalpy of formation, heat capacity, and entropy in the sas, liquid, and solid phases. Past efforts in this area have provided about 450 group contribution values for estimation of the enthalpy of formation of Iiquids and solids at $298 \mathrm{~K}$. During the current year, work was completed on group contribution value calculation for the heat capacity and entropy in the liquid and solid phases at $298 \mathrm{~K}$. Evaluation of group contribution values is underway in order to bring about consistency among given groups as estimates are made to calculate values for a compound in the gas, liquid, and solid phases.

A major activity has been the preparation of the prototype edition of the CODATA Thermodynamic Tables. These are the first of a new international set of thermodynamic tables. These comprehensive tables contain contributions from groups in four countries: thermochemistry (NBS and the Institute for High Temperatures (IVTAN), Moscow), thermal functions (the JANAF group at Dow Chemical, Midland, Michigan, and IVTAN), alloys (ENSEEG, University of Grenoble, France), fused salts (AERE, Harwell, United Kingdom), and aqueous solutions (NBS). This edition is devoted to compounds of calcium. This Data Center has analyzed some 375 measurements on calcium species and provided recommended values for their thermochemistry. In parallel the Electrolyte Data Center has prepared data on the aqueous solutions of calcium chloride. In addition all of the technical coordination, editorial work, and production of the tables has been done here. The tables were submitted in August 1984 to the CODATA Task Group on Chemical Thermodynamic Tables for final review and subsequent publication.

A second major evaluation activity has been the correlation of data on aqueous solutions of sulfur dioxide, a joint activity with the Electrolyte Data Center. A set of recommended values has been prepared that covers vapor-liquid equilibria and ionization for processes in solution. 


$$
\begin{aligned}
& \mathrm{SO}_{2}(\mathrm{~g})=\mathrm{SO}_{2}(\mathrm{aq}) \\
& \mathrm{SO}_{2}(\mathrm{aq})+\mathrm{H}_{2} \mathrm{O}=\mathrm{H}^{+}(\mathrm{aq})+\mathrm{HSO}_{3}^{-}(\mathrm{aq}) \\
& \mathrm{HSO}_{3}^{-}(\mathrm{aq})=\mathrm{H}^{+}(\mathrm{aq})+\mathrm{SO}_{3}^{-}(\mathrm{aq}) \\
& \mathrm{HSO}_{3}^{-}(\mathrm{aq})=\mathrm{H}_{2} \mathrm{O}+\mathrm{S}_{2} \mathrm{O}_{5}^{-}(\mathrm{aq})
\end{aligned}
$$

The temperature range $25-100^{\circ} \mathrm{C}$ is covered. Recommendations are made for enthalpies of the processes, equilibrium constants, and activity coeficlents. New, more precise measurements and analysis using our weak electrolyte modelling codes have led to substantially improved recommendations. One result is an appreciable change in the $\Delta_{\mathrm{f}} \mathrm{H}$ and $\Delta_{\mathrm{f}} \mathrm{G}$ of $\mathrm{SO}_{3}(\mathrm{aq})$, a situation that may have an impact on tabulated data for many metal sulfides. These new evaluations were prepared for the DoE flue gas desulfurization program, the DIPPR Electrolyte Handbook, and OSRD. Additional detail is given in the section on electrolyte data.

New recommendations for thermochemical properties of $\mathrm{Sr}^{+2}(\mathrm{aq})$ and several strontium salts have been made, jointly with researchers at the U.S. Geological Survey. New recommended values for the enthalpy of combustion of components of natural gas have been prepared, jointly with workers of the Thermodynamic Research Center, Texas A\&M Universicy. The estimation of enthalpies of formation, heat capacities, and entropies of liquid and solid organic compounds continues, is being coordinated with work at the University of Sussex, U.K.

The preparation of numerical data bases, in machine readable form, is a major task in the modern technique for evaluating thermochemical data. These data-bases of measurements are used as input to programs that calculate enthalpies and Giobs energies of formation and entropies and are also used to document recommended values. Three data-bases were prepared or extended during the year. One is the set of measurements on calcium compounds for the CODATA Tables, mentioned above. The second is a catalog of measurements documenting the CODATA Key VaIues for Thermodynamics. This very important data base covers the substances upon which many data evaluations hang and will be the basis for future CODATA Key values activities. The third is a data-base covering compounds of sulfur with hydrogen or oxygen (or both) that are of interest in ilue gas desulfurization problems. It includes measurements published through 1983 and is a start on updating the CODATA recommended values into the "NBS Tables of Chemical Thermodynamic Properties."

2. Accessions, Information and Sucport

(T. L. Jobe, E. D. Hearing, and C. Jackson)

These are onzoing activities that make possible the systematic evaluation of data. During the year 2800 articles have been located in the current literature, abstracted and entered in our index to thermodynamic measurements. This work produced 14,000 index entries and will te the basis for the Inorganic Substances section of volume 27 of the bulletin of Chemical Thermodynamics. Information activities during the 
year have emphasized a joint project with the Electrolyte Data Center, preparation of a series of bibliographies on the thermodynamics of aqueous solutions.

During FY 1984, Joyce J. Grimes transfered from the Chemical Thermodynamics Data Center to the NBS Equal Employment Opportunity Support Division.

3. Autcmation Activities

(D. Bickham, R. Coyne, P. Fagan, and D. B. Neumann)

Four data centers share the use of a mini-computer (HP-1000) via terminals and interfaced microcomputers. The principal use of the HP-1000 is word processing and data manipulation. Calculations are a very small fraction of the work load. Individual researchers reduce data, model systems and prepare graphic output. All of these users are supported by the automation group of the Chemical Thermodynamics Data Center which is responsible for systems maintenance and development. The group is aided by individuals who have particular needs for special utilities.

An exciting, new activity is the development of a chemically oriented data base management system suitable for use by four CCP data centers (Chemical Thermodynamics, Electrolyte, Chemical Kinetics, Ion Energetics). This is a multi-year effort, part of which has been completed. The entire system will contain packages that accept, check, edit, store and retrieve chemical nomenclature (in a broad sense), chemical reactions and associated quantitative data, bibliography, subject abstracts, reccmmended physiochemicai data and interiaces to calculated programs.

This year our work focused on the bibliographic and abstracts portions of the system. The bibliographic system, originally designed for use in thermochemistry, has been expanded to meet the demands of other data centers and also to include the ANSI standards on bibliographic format. This work is being done jointly with the programming staff of the Office of Standard Reference Data. The resultant programs will be tested and applied widely in the OSRD system. The present system has been applied successfully in the preparation of the CODATA Tables and the DIPPR bibliographies.

Subject abstracts have been prepared in autated form since 1970 in the Chemical Thermodynamics Data Center. The files have been processed to zive hardcopy for our index to thermodynamic measurements and for the Bulletin of Chemical Thermodynamics. During this year the automation effort has been devoted to implementing a system to retrieve the accumulated abstracts in a form suitable for loading into a data base management system. The first phase is complete. Retrieval has been achieved and the output form specified. Programning is proceeding to provide for loading into the DBMS. One spin-off from this work is the preparation of a transferable program for processing the current subject abstracts. It is being provided to the editor of the Bulletin of Chemical Thermodynamics for use in the preparation of copy and in the development of derivative daca-bases. 
B. Electrolyte Data Center

(R. N. Goldberg and D. Smith-Magowan)

The Electrolyte Data Center serves as a resource for correlated data on properties of solutions of aqueous electrolytes and for compilations of data. The Data Center provides, on a continuing basis, information services and standard reference data for selected thermodyanmic properties of aqueous solutions of electrolytes. It provides methodologies for correlation and estimation of the properties of classes of electrolytes.

Three problem areas that are addressed directly by this electrolyte evalution program are environmental quality, energy, and health. Data are needed in laboratory research, water pollution studies, advanced battery design, water and sewage processing, corrosion control, flue-gas washing processes, geothermal energy systems, bictechnology, oceanography and specialized electrolytic applications such as analyses using ion-selective electrodes.

There are four areas into which recent activities can be grouped: (1) a correlation of the thermodynamics of the $\mathrm{SO}_{2}+\mathrm{H}_{2} \mathrm{O}$ system. (2) development of procedures and algorithms for the modeling of complex aqueous solutions, (3) development of a data base of activity and osmotic coefficients and of programs for the manipulation and use of the data base, and (4) completion of specific bibliographies of industrial interest.

\section{Thermodynamics of the $\mathrm{SO}_{2}+\mathrm{H}_{2} \mathrm{O}$ System}

A manuscript written in collaboration with $V$. Parker which summarizes the thermodynamics of two $\mathrm{SO}_{2}+\mathrm{H}_{2} \mathrm{O}$ System Ions has been prepared and is presently in review. The results of this manuscript are summarized herein. A consistant set of thermochemical property values, $\Delta_{\mathrm{f}} \mathrm{H}^{\circ}, \Delta_{\mathrm{r}} \mathrm{G}^{\circ}, \mathrm{S}^{\circ}$, and $\mathrm{C}_{\mathrm{p}}^{\circ}$ at 298.15 K were selected for the constituents of aquecus $\mathrm{SO}_{2}$ $\left(\mathrm{SO}_{2}(\mathrm{aq}), \mathrm{HSO}_{3}^{-}(\mathrm{aq}), \mathrm{SO}_{3}^{2-}(\mathrm{aq}), \mathrm{H}^{+}(\mathrm{aq})\right.$, and $\mathrm{S}_{2} \mathrm{O}_{3}^{2-}(\mathrm{aq})$ ). Also tabulated are values of MO meunionic activity coefficients, osmotic coefficients, partial pressures of $\operatorname{SOP}_{2}(8)$, and the relative apparent nolar enthalpy as a function of the stoichimetric concentration of $\mathrm{SO}_{2}$ (aq) at $298.15 \mathrm{~K}$. The analysis of the data considered a wide variety of measurement techniques: calorimetric enthalpies of solution and reaction, heat capacities equilibrium constants, solubilities, and vapor pressure measurements, both partial and total, over the temperature range 278 to $393 \mathrm{~K}$. All auxiliary data was taken from the most recent sez of CODATA values which were converted to a standard state pressure of 1 bar ( 0.1 $\mathrm{MPa})$. For the proces $\mathrm{SO}_{2}(\mathrm{~g})=\mathrm{SO}_{2}(\mathrm{aq})$, the selected "best" va? ues are: $\mathrm{K}=1.22 \pm 0.05 \mathrm{~mol} \mathrm{~kg} \mathrm{kgr}^{-1} \mathrm{bar}^{-1} \Delta \mathrm{G}^{0}=-0.50 \pm 0.10 \mathrm{~kJ} \mathrm{mcl}^{-1}$, and $\Delta \mathrm{H}^{\circ}=$ $-27.18 \pm 0.20 \mathrm{~kJ} \mathrm{~mol}^{-1}$ at $298.15 \mathrm{~K}$. The standard state partial molar entropy of $\mathrm{SO}_{3}^{2-}(\mathrm{a})$ is $-15.30 \pm 0.70 \mathrm{~J} \mathrm{~mol}^{-1} \mathrm{~K}^{-1}$ at $298.15 \mathrm{~K}$. This ralue 
was obtained by the analysis of data via two independent thermodyanmic pathways. Parameters are given which extend the predictions to temperatures up to $373 \mathrm{~K}$.

\section{Data Base of Activity and Osmotic Coefficients}

A data-base of activity and osmotic coefficients and excess Gibbs energies for approximately 400 aqueous binary electrolyte solutions has been assembled. The data base contains, for each electrolyte solution, the coefficients of one or more of ten different correlating equations. The data base has been assembled into EORTRAN "DATA" statements so that it can be compiled as a subroutine and then linked with other codes for efficient use. Programs have also been written which can be used to calculate values of the activity and osmotic coefficients at mobelities specified by the user. The programs can be used ei ther interactively or in batch modes of operation. Before this package (which will be made available on magnetic tape) can be released, there must be final assembly of the data base and the algorithms accompanying it, a thorough check of its operation, and documentation must be written.

\section{Modeling of Complex Aqueous Solutions}

This activity was described in detail in our last two panel reports. A lengthy manuscript describing the procedures is currently in press. These same procedures have also been applied to the modelling of two $\mathrm{SO}_{2}+\mathrm{H}_{2} \mathrm{O}$ systems (see above). Progress this past year on the development of the codes and procedures have included the following: (1) a very careful structuring of the 23 subroutines used in the calculations which permits one to modify or to extend the codes in an efficient manner, (2) an extension of the codes to allow for a much larger number of species to be treated in two equilibrium calculations (The actual number is now machine and not algorithm dependent), and (3) a demonstration that hydration effects coupled with the introduction of the species CuSO ${ }_{4}^{\circ}$, $\mathrm{Cu}_{2} \mathrm{SO}_{4}^{2+}$, and $\mathrm{Cu}\left(\mathrm{SO}_{4}\right)_{2}^{2-}$ in the equilibrium model can be used to represent the thermodynamic behavior of aqueous copper sulfate solutions up to a mobility of at least one mol $\mathrm{kg}^{-1}$.

While the algorithms can in principal handle very large numbers of species, the actual solution of the simultaneous non-linear equations which describe the equilibria in solution has been found to be highly dependent upon providing good initial estimates of the sought after parameters when treating systems containing more than about ten different species. There are several approaches which can be taken to solve this problem. They are: (1) improved numerical scaling of the parameters, (2) heuristic search procedures, and (3) a brute-force approach in which the n-dimensional space containing all possible solutions is systematically searched for the correct answers. In any case, a reasonable solution to this problem must be round before we are able to deal with the kinds of larger scale systems which we would like to treat in the future. 
This kind of procedure is particularly applicable to solutions which display lon-pairing, complexation, and hydrolysis behavior. These are the kind of phenomena which become more significant at high temperatures. A logical application and next step in the use of these codes is to regress the parameters of the model itself from measurement data (Gibbs energies, enthalpies, and heat capacities) on solutions over a wide range of temperature.

\section{Bibliographies of Industrial Interest}

Elght bibliographies of thermodynamic data pertinent to aqueous solutions have been completed. They are: $\mathrm{CO}_{2}, \mathrm{H}_{2} \mathrm{~S}, \mathrm{H}_{2} \mathrm{SO}_{4}, \mathrm{NH}_{3}, \mathrm{CuCl}_{2}$, $\mathrm{H}_{3} \mathrm{PO}_{4}, \mathrm{ZnCl} 2$, and $\left(\mathrm{H}_{2} \mathrm{~S}+\mathrm{CO}_{2}, \mathrm{H}_{2} \mathrm{~S}+\mathrm{NH}_{3}, \mathrm{CO}_{2}+\mathrm{NH}_{3}, \mathrm{CO}_{2}+\mathrm{H}_{2} \mathrm{~S}+\mathrm{NH}_{3}\right)$. Papers included in these bibliographies pertain to vapor-liquid-equilibria, the thermal and excess properties of these solutions, and equilibria in solution. These blbliographies were sponsored by the Design Institute for Physical Property Data of the American Institute of Chemical Engineers.

\section{Future Plans}

This next year we will begin development of a machine data-base of the evaluated information gathered on $\Delta_{f} H, C_{p}$, and $S$ at $298 \mathrm{~K}$ for amino acids, peptides and heterocyclic compounds so that tables of data can be derived from an appropriate algorithm. We will prepare a manuscript containng group contribution values for estimating $\Delta_{\mathrm{f}} \mathrm{H}, \mathrm{C}_{\mathrm{p}}$ and $\mathrm{S}$ of organic compounds in the gaseous, liquid, and solid phases at $298 \mathrm{~K}$.

Our longer range plans include the supplement of the CODATA Key Values for Thermodynamics with data on substances commonly used in science and industry and for which there are high-quality thermochemical measurements. Addition of data on a thousand substances is the goal. As a necessary and much needed compliment to the evaluation work we will develop new methods for predicting values of properties based on molecular and crystal structure, lattice energies, similarities within classes inorganic of compounds and their position in the periodic tables. Prepare reaction catalogs needed by CODATA and documenting "the NBS tables" and publish these.

Next year our automation activities will deliver a fully operational bibliographic retrieval system. We should aso be in a position to implement a prototype search and retrieval system for subject abstractz and reaction catalog data. We will also onvert an existing organic thermochemistry retrieval system to operation on the iocal HP-1000 system. We also plan to add selected calculation and graphics to the HP-1000 system in support of thermochemical data evaluation and upgrade the minicomputer system to the HP-1000 A 900 series. During the year we will expand the NBS index to thermodynamic measurements by selecting and abstracting papers published in 1984-85, prepare the inorganic thermochemistry section for volume 28 of the Bulletin of Chemical Thermodynamics, and provide information services in response to request. 
In the Division's efforts to make the Chemical Thermodynamics Data Center preeminent source for thermochemical data, world-wide five activities are being undertaken. They are:

1. Maximize the amount of evaluated thermochemical data compatible with the CODATA Key Values and at the same time document the NBS Tables of Chemical Thermodynamic Properties. The principal mechanism is the construction and use of catalogs of measurements used by data evaluators in preparing tables.

2. Define a key network of thermochemical data and keep current the evaluated data for it. This network will include industrially important processes, those essential for thermodynamic data evaluation, and those of major scientific concern, e.g., geochemistry, molecular structure studies, biochemistry, etc.

3. Participate in the joint preparation of tables of thermodynamic data by international and national groups. This is our preferred route to rapid expansion of the stock of up-to-date evaluated thermodynamic data. The principal mechanism will be participation in the work of the CODATA Task Group on Chemical Thermodynamic Tables (and development of a leadership role there), and bilateral exchange agreements or collaborations with institutions in the U.S.S.R., the U.K. and in the U.S.A.

4. Design and implement a thermochemical information system which includes a rapid reply system, (based on our files), updates to CIS, addition of the JANAF Tables to our files and CIS, and, very important, a suite of programs to calculate properties of reactions.

5. Develop predictive methods for thermodynamic properties. This is an activity to extend existing data-bases, and also to let us select among divergent data. Methods are needed for both organic and inorganic compounds. 
(a) Publications of Past Year

Abramowitz, S., "Barriers to Internal Rotation in Inorganic Species," Thermochemistry and Its Applications to Chemical and Blochemical Systems, NATO Advanced Study Institute, Series C, Mathematical and Physical Sciences, Vol. 119 (M.A.V. Ribeiro da Silva, Editor; D. Reidel Publishing Co.), 789-802 (1984).

Abramowitz, S., Domalski, E.S., Churney, K.L., Ledford, A.E., Ryan, R.V., and Re1lly, M.L., "An Oxygen Flow Calorimeter for Determining the Heating Values of Kllogram-size Samples of Municipal Solid Waste," Thermochemistry and Its Applications to Chemical and Biochemical Systems, NATO Advanced Study Institute, Series C, Mathematical and Physical Sciences, Vol. 119 (M.A.V.Ribelro da Silva, editor; D. Reidel Publishing Co.), 61-76, (1984).

Abramowitz, S., Wagman, D.D., Parker, V.B., and Garvin, D., "Critical Evaluation of Thermodynamic Data - A Research Activity," Thermochemistry and Its Applications to Chemical and Biochemical Systems, NATO Advanced Study Institute, Series C, Mathematical and Physical Sciences, Vol. 119 ( M.A.V. Ribelro da Silva editor; D. Reidel Publishing Co.), 803-814, (1984).

Abramowitz, S., "Barriers to Internal Rotation in Inorganic Species," Proceedings of High Temperature Chemistry Symposium, Bombay, India, January $28-30,789-802,(1983)$.

Armstrong, G.T., and Jobe, T.L., Jr., "Heating Values of Natural Gas and Its Components: Conversion of Values to Measurement Bases and Calculation op Mixtures," Stationary Gas Turbine Alternative Fuels, ASTM ST? 809, (J.C. Clark and S.M. DeCorso, Eds., American Society for Testing and Materials, Philadelphia), 314-334, (1983).

Churney, K.L., Domalski, E.S., Ledford, Jr., A.E., Colbert, J.C., Bruce, S.S., Buckley, I.J., Paule, R.C., Reilly; M.L., "Assessing the Credibility ol" the Calorific Value of Municipal Solid Waste," NBSIR 34-2825 (DoE), February, (1984).

DeVoe, H. and Wasik, S.P., Aqueous Solubilities and Enthalpies of Solution of Adenine and Guanine," J. Solution Chem. 13, 51-60 (1984).

Ditmars, D.A., "Heat-capacity Calorimetry by the Method of Mixtures," Chap 13 , Compendium of Thermophysical Property Measurement Methods, I, (K.D. Maglic, Editor; ?lenum ?ublishing Corporation.) 527-553 (1984).

Dizdaroglu, M., Gajewski, E., Simis, M.G., "Enzymatic Digestibility of Peptides Crosslinked by Ionizing Radiation," Int. J. Radiat. 3101. 45 (3), 283-295, (1984). 
Domalski, E.S., Evans, W.H., and Hearing, E.D., "Heat Capacities, Entropies and Some Phase Transition Properties of Organic Compounds in the Liquid and Solid Phases," J. Phys. Chem. Ref. Data 13, Supplement No. 1, $1-26$ (1984).

Fuger, J., Parker, V.B., Hubbard, W., Oetting, F., "The Chemical Thermodynamlcs of Actinide Elements and Compounds. Part 8. The Actinide Halides," IAEA, Vienna, Austria 1-267 (1983).

Goldberg, R.N., "An Equilibrium Model for the Calculation of Activity and Osmotic Coefficients in Aqueous Solutions, " J. Research NBS, 89, No. 3 , 251-263 (1984).

Kirklin, D.R., and Domalski, E.S., "A 25 Gram-Capacity Oxygen Bomb Calorimeter," Resources \& Conservation 10 177-187 (1983).

Kirklin, D.R., and Domalski, E.S., "Enthalpy of Combustion of Adenine," J. Chem. Thermodynam. 15 941-947 (1983).

Kirklin, D.R., and Domalski, E.S., "Enthalpy of Combustion of Purine," J. Chem. Thermodynam. 16 633-641 (1984).

Miller, M.M., Ghodbane, S., Was1k, S., Tewari, Y.B., Martire, D.E., "Aqueous Solubilities, Octanol/Water Partition Coefficients, and Entropies of Melting of Chlorinated Benzenes and Biphenyls," J. Chem. Eng. Data 29, 184-190 (1984).

Tewari,. Y.B., and Goldberg, R.N., "Thermodynamics of the Conversion of Aqueous Glucose to Eructose," J. Solution Chem. 13, 523-547 (1984). 


\section{(b) Publications in Progress}

Abramowitz, S., "Biotechnology and Bioseparations, Thermodynamic Needs of the Decade Ahead," National Science Foundation, Workshop Proceedings, 1983, (in press).

Busenberg, E., Plummer, L.N., Parker, V.B., "The Solubility of Strontianite $\left(\mathrm{SrCO}_{3}\right)$ in $\mathrm{CO}_{2}-\mathrm{H}_{2} \mathrm{O}$ Solutions Between 2 and $91{ }^{\circ} \mathrm{C}$, the Association Constants of $\mathrm{SrHCO}_{3}^{+2}$ and $\mathrm{SrCO}_{3}$ Between 5 and $80{ }^{\circ} \mathrm{C}$ and an Evaluation of the Thermodynamic Properties of $\mathrm{Sr}^{2+}(\mathrm{aq})$ at $25^{\circ} \mathrm{C}$ and 1 Atmosphere," Acta Geochimica, (in press).

Domalski, E.S., Churney, K.L., Ledford, Jr., A.E., Colbert, J.C., Bruce, S.S., Buckley, T.J., Paule, R.C., Reilly; M.L., "Determining the Calorific Value of Municipal Solid Waste: 'Experimental Results From a 2.5 Kilogramcapacity Flow Calorimeter," Proceedings of Energy from Municipal Waste Research Workshop, Kissimmee, Florida. February 22-24, 1984, (in press).

Gajewsk1, E., Dizdaroglu, M., Krutzsch, C., and Simic, M.G., "OH RadicalInduced Dimerization and Racemization of Methionine in Peptides." Int. J. Radiat. Biol., (in press).

Garvin, D., Goldberg, R.N., and Staples, B.R., "Evaluated Data and Data Bases: Chemical Thermodynamics in the Steam Power Cycle," Proceedings of the Workshop in Steam Power Cycles Data Requirements, $H$. White and 0 . Jonas, Editors, (in press).

Garvin, D., Staples, B.R., and Jobe, Jr., T.L., "A Blbliography on the Equilibria in the $\mathrm{CuCl}_{2}-\mathrm{H}_{2} \mathrm{O}$ System," Handbook of Aqueous Thermodynamics, Design Institute for Physical Property Data, (DIPPR), (submitted).

Goldberg, R.N. and Parker, V.B., "Thermodynamics of Solution of $\mathrm{SO}_{2}(8)$ in Water and of Aqueous Sulfur Dioxide Solutions," (submitted).

Goldberg, R.N. and Steckler, D.,"A Bibliography of Sources of Thermodynamic Data for the System: $\mathrm{CO}_{2}+\mathrm{NH}_{3}+\mathrm{H}_{2} \mathrm{O}, \mathrm{CO}_{2}+\mathrm{H}_{2} \mathrm{~S}+\mathrm{H}_{2} \mathrm{O}, \mathrm{H}_{2} \mathrm{~S}+\mathrm{NH}_{3}+\mathrm{H}_{2} \mathrm{O}$, $\mathrm{CO}_{2}+\mathrm{NH}_{3}+\mathrm{H}_{2} \mathrm{~S}+\mathrm{H}_{2} \mathrm{O}$ ", Handbook of Aqueous Thermodynamics, Design Institute for ?hysical Property Data (DIPPR), (submitted).

Goldberg, R.N., "Compiled Thermodynamic Data Sources for Aqueous and $310 c h e m i-$ cal Systems: An Annotated Bibliography (1930 to 1983)," Handbook of Aqueous Thermodynamics, Design Institute for Physical Property Data (DIPPR), (submitted).

Jobe, Jr., T.L., and Coldberg, R.N., "A Bibliography of Sources of Thermodynamic Data for Aqueous Zinc Chloride Solutions," Handbook of Aqueous Thermodynamics, Design Institute for Physical Property Data, (DIPPR), (submitted). 
Jobe, Jr., T.L., and Goldberg, R.N., "A Bibliography of Sources of Thermodynamic Data for Aqueous Phosphoric Acid Solutions," Handbook of Aqueous Thermodynamics, Design Institue for Physical Property Data, (DIPPR), (submitted).

Kirklin, D.R., Decker, P.H., and Domalski, E.S., "Characterization of Refuse-Derived Fuel at Various Stages of Processing," Resources and Conservation, (submitted).

Simic, M.G., Gajewski, E., and Dizdaroglu, M., "Kinetics and Mechanism of Radiation-induced Dimerization of Phenylalanine Peptides," J. Phys. Chem. (in press).

Tewari, Y.B., and Goldberg, R.N., "A Note on the Thermodynamics of the Conversion of Aqueous Glucose to Fructose," Appl. Biochem. \& Biotech., (in press).

Wasik, S.P., Schwarz, F.P., Tewari, Y.B., Miller, M.M., Purnell, J.H., "A Head-Space Method for Measuring Activity Coefficients, Partition Coefficients, and Solubilities of Hydrocarbons in Aqueous Solutions," J. Research NBS, 89, (in press).

Miller, M.M., Wasik, S.P., Guang, G.L., Shui, W.Y., and MacKay, D., "Relationships Between Octanol-water Partition Coefficient, Aqueous Solubility and Bioconcentration Factors, (submitted). 


\author{
A. Weber, Chief \\ SUMMARY OF ACTIVITIES
}

Fiscal Year 1984

1. Introduction

A. Goal

This Division is a discipline oriented unit and its goal is to be at the forefront of modern theoretical and experimental spectroscopy in order to:

Advance spectroscopic measurement methods and techniques

Develop models, theoretical concepts and quantum theoretical methods for predicting molecular properties and interactions

Obtain reliable reference spectroscopic data for NBS, government, research community and industrial needs

Understand and model fundamental chemical processes

Provide state-of-the-art frequency standards

Transfer forefront spectroscopic expertise to other NBS Divisions, other government agencies, industry and the research community.

Within the framework of these general goals the Division also follows three specific thrusts which are part of the overall program of the Center for Chemical Physics - Properties of Weakly Bonded Systems, Biotecinology, and Picosecond Spectroscopy. Properties of weakly bonded systems are obtained for small atomic and molecular complexes held together by van der Waals, hydrogen bond, and other weak forces in order to develop a cata base to understand conformation, thermodynamics, and reactivity of biochemical systems. The objectives of our thrust in biotechnologj are to develcp and implement methods for the study of metal interactions in proteins and nucleic acids using primarily quantum mechanical techniques, while the major effort of the picosecond thrust has been directed to the study of vibrational energy transfer.

This summary contains a report of the activities of each of the three groups within the Division during the past year and demonstrates now they are meeting the above objectives. 
To carry out its work the Molecular Spectroscopy Division is organized into three working groups: High Resolution Spectroscopy (HRS), Laser Photochemistry (LPC), and Quantum Chemistry (QC). The permanent Division staff is augmented by that of postdoctoral fellows and guest workers. During the past year we have had twelve guest workers and six postdoctoral fellows engaged in Division projects. A list of the guest workers, their permanent affiliations, and their activities is given in part 10 of this report.

\section{Needs of the User Community}

With the technological advances of micro- and main frame computers and the development of tunable dye and tunable diode lasers that have taken place during the last eight years, the fields of experimental molecular spectroscopy and theoretical quantum chemistry have grown rapidly.

To be able to respond to the present and projected needs of NBS, the Center for Chemical Physics (CCP), and the user community, a significant fraction of the Division's effort must be directed toward achieving and maintaining the expertise of its staff in their discipline oriented fields, and into maintaining its experimental facilities at the state-of-the-art level. We must remain at this level of expertise in spectroscopic measurements, theory, and interpretation in order to respond quickly to provide standards, serve national technical needs, and to insure adequate response to future, unknown problems. The soundness of this approach is justified cy the past and present applications of experimental and theoretical spectroscopy by our Division. These applications also provide much of the direction and motivation for our efforts. Remote sensing for industrial and atmospheric monitoring, laser isotope separation and fusion, laser chemistry, and combustion diagnostics are a few of the many important high-technology areas that depend on modern spectroscopic techniques.

\section{Interactions with the User Community}

In order to meet the calibration and standards requirements for the design and application of tunable infrared lasers and Fourier transform instrumentation over the next decade, several frequency standards activities are pursued in collaboration with several groups from within and outside NBS. Details about our Calibration Standards work are given in Sections $B$ and $H$ of the HRS group repcrt.

We continue to be involved in the spectroscopy of the environmental and space sciences. Our work is directed toward the broadening of the data base upon which spectroscopic measurements of trace atmospheric constituents by means of ground based, balloon and satellite borne instruments are performed and evaluated. This, as well as similar efforts of interest to various branches of NASA and other user groups, is part of 
our continuing involvement in problems of national concern. Details about these and other activities may be found in Section $C$ of the HRS group report.

Our Division has significant other agency contracts with ONR, ARO, AFOSR, DOE, NASA, CMA, and the National Foundation for Cancer Research that help these agencies fulfill their missions. Details are discussed in the group reports. These efforts along with the lists of consultations, talks, publications, and collaborative activities show our strong coupling with industry, other government agencies, and the research community.

The above constitutes only a sample of our involvement and response to the needs of the user community. Our Division is host to several guest workers from universities, other government agencies, and from overseas countries (see section 10). The numerous and varied interactions and collaborations of the Division's staff with non-NBS scientists and user groups are detailed in part 9 - Professional Interactions, Consulting and Advisory Services.

\section{E. Interactions within NBS}

One of our principal objectives is to provide major spectroscopic expertise to other NBS divisions and programs.

A project on spectral frequency standards is undertaken jointly by scientists from the Time and Frequency Division of NBS/Boulder and members of the.HRS group. These activities are described in sections $B$ and $H$ of the HRS group report.

We have completed the second year of a new program in the spectroscopy of condensed phase systems. This effort is part of an overall program of the Center for Chemical Physics' new emphasis on condensed phase chemistry, including selective areas of biotechnology. The Division's participation is in the areas of picosecond siudies, quantum chemical investigations of the interaction of metal atoms with biomolecules, molecule-surface interactions, high resolution spectroscopy of hydrogen-bonded molecules, and metal clusters. These activities are described in the individual group reports. To augment the experimental facilities for microwave spectroscopy in order to study the spectra of weakly bonded complexes, a new pulsed beam, Fabry-Perot cavity, Fourier transform microwave spectrometer was constructed during the past year. Details about this instrument and its performance are given in sectior $G .3$ of the HRS group report.

The field of Quantum Chemistry has in recent years experienced significant new developments which extend the range or reliable and usefui calculations to complex polyatomic systems. A new program to attack the problems of the stabilities, conformations, spectroscopy and reactivity of complex molecules, clusters, ions, radicals, and transition states, amons others, was successfully conducted for the second year by the Quantum Chemistry grcup. 


\section{High Resolution Spectroscopy Group}

J. T. Hougen, B. J. Howard, W. J. Lafferty, R. W. Lovejoy, F. J. Lovas, A. G. Maki, N. Ohashi, W. B. Olson, A. S. Pine, M. Raab, G. Rotter, R. D. Suenram, A. Weber

\section{A. Introduction}

The work of this group involves experimental and theoretical applications of molecular spectroscopy for characterizing gas phase molecules, primarily in the infrared (rotation-vibrational) and microwave (rotational) spectral regions, the development of frequency standards for the infrared, and the development and application of new experimental techniques for high resolution spectroscopy. The emphasis of these studies is in the applications of our expertise to problems in structural chemistry, atmospheric chemistry, chemical analysis, radio astronomy and the properties of novel molecular species, particularly floppy molecules. These investigations are made possible through the diverse instrumentation (difference frequency laser spectrometer, high resolution Fourier transform spectrometer, pulsed beam Fabry-Perot cavity Fourier transform microwave spectrometer) available in the division.

Molecular radio astronomy in recent years has generated a very pronounced demand for microwave laboratory data and spectroscopic expertise. A portion of the current measurement and analysis effort is aimed at providing the important data needed to complete the reviews of spectra of known interstellar molecules described in Section $\mathrm{H}$ of this report. Our collaborations with radio-astronomers provide the contacts and experience necessary to insure that these reviews are both practical and useful.

The major activity of the data center is to provide evallated data on molecular rotational spectra in the microwave region and to develop evaluated infrared spectra to be used as standards in calibrating diode lasers and infrared laser spectrometers. This work is based on the needs of the user community. Thus the work on infrared standards is a response to requests from the instrumentation industry, industrial research laboratories, applied technological user groups, and government agencies and laboratories.

Sustained collaboration with non-NBS groups serves to disseminate the results of our work in the quickest and most far-reaching way to the user community. Such collaboration also benefits our work through access to data and facilities not presently available at NBS. Particuiarly noteworthy among the non-NBS institutions with which beneficial collaborations have occurred during the past year are: The University of oulu, Oulu, Finland, the University of Illinois, the University of Alberta, the National Research Council of Canada, the Harry Diamond Laboratories of the U.S. Army, the NASA-Greenbelt, NASA-Langley, NASA-Goddard, and NASA-Ames Laboratories, and the Kitt Peak National Observatory. 
Our group's scientists are also aware of the need to serve the scientific community at large. This they do in a variety of ways, including membership and active participation in national and international committees, workshops and review panels. This activity is listed elsewhere in this report (see Sections 8 and 9).

The various technical activities of the High Resolution Spectroscopy group for the period covered by this review are described under the following seven, broad categories (B-H), each of which lists several projects. Names of group members active in a project are listed at the beginning of each project's description.

\section{B. Development of Calibration Standards for the Infrared Spectrum}

The development of frequency standards for the infrared spectral region is a long term project of the group. Until this project was undertaken most of the available frequency standards had uncertainties between \pm 0.002 and $\pm 0.005 \mathrm{~cm}^{-1}$. Since this is an order of magnitude worse than their measurement capability, many users of modern higin resolution tunable laser systems and other high-performance spectroscopic devices have asked us for advice on calibrating their measurements. In many cases we have been able to help them to our mutual satisfaction, but in some cases the available calibration sources have been unsatisfactory and the inquiries have only served to spur our efforts by demonstrating the need.

Our strategy in developing calibration standards has been to follow the precedent already set by IUPAC (International Union of Pure and Applied Chemistry) in providing calibration for lower resolution devices. These standards consist of tables of frequencies for absorption line spectra of inexpensive, easily handled, and readily available gases. Such standards do not require expensive additional equipment, are easily and accurately reproduced, and can be quickly utilized by relatively untrained personnel.

The best standards are provided by substances that give single Iine spectra when measured under conditions giving Doppler limited resolution. Ideal candidates have at least one calibration quality feature at every $i \mathrm{~cm}^{-i}$ interval and preferably more. We prefer to use substances that have a well behaved energy level pattern so that unmeasured transition frequencies and their uncertainties may be calculated with confidence from a minimum set of physical parameters that have been very accurately measured. We also prefer to use substances for which the necessary physica? parameters have been measured by several laboratories. Erequency teasurement techniques for determining the physical parameters are preferred over length or wavelength measurement techniques because frequency measurements are generally less susceptible to unexpected systematic errors.

So far our efforts have been corcentrated on the molecular species CO, OCS, $\mathrm{N}_{2} \mathrm{O}_{3}, \mathrm{C}_{2} \mathrm{H}_{2}$, and $\mathrm{CO}_{2}$. Others have used $\mathrm{HCl}, \mathrm{HE}$, $\mathrm{NH}_{3}, \mathrm{HCH}$, and $\mathrm{H}_{2} \mathrm{O}$. We have paid particular attention to systematicaliy developing a se: of molecular parameters that would enable us to calculate unneasured transitions with a reliably predictable uncertainty. The difficulty of 
reliably estimating the uncertainty of certain transitions has caused us to avoid using more complex molecular species until we have exhausted all calibration possibilities using the simpler species.

During the past year our efforts have concentrated on a) preparing a review article on calibration data available for tunable laser devices, b) completing publication of the measurements on $\mathrm{DBr}$ (calibration 50-200 $\mathrm{cm}^{-1}$ and 1595-1990 $\mathrm{cm}^{-1}$ ) and $\mathrm{N}_{2} \mathrm{O}$ (calibration 4340-4755 $\mathrm{cm}^{-1}$ ), c) measuring the pressure induced frequency shift of $\mathrm{NO}_{3}$ and d) continuing the analysis of the FTS measurements on OCS, $\mathrm{N}_{2} \mathrm{O}$, and $\mathrm{CO}_{2}$ from 450 to 720 $\mathrm{cm}^{-1}$.

1. New Measurements on $\mathrm{N}_{2} \mathrm{O}$ (A.G. Maki)

In our continuing collaboration with Joe Wells and others in the Time and Frequency Division at NBS-Boulder, new heterodyne measurements have been made on the $01^{1} 1-000_{0}$ band of $\mathrm{N}_{2} \mathrm{O}$ near $1880 \mathrm{~cm}^{-1}$ using a CO laser as a frequency transfer standard. These measurements, coupled with frequency measurements on rotational transitions in both the upper and lower states of $\mathrm{N}_{2} \mathrm{O}$, give us an excelient grid of frequency standards from 1830 to $1920 \mathrm{~cm}^{3}$.

This is a particularly valuable band from which to obtain frequency measurements as it can be used as a test on the accuracy of the Fourier transform spectra which we have obtained from Dr. Kauppinen of Oulu University, Finland. That is to say, the sum of the frequencies $01^{1} 1$ $-00_{1}^{0}, 000_{1}-01^{1} \mathrm{O}$, and $01^{1} 0$ - $00^{0} 0$ must equal the frequency of $01^{1} 1$ $-00^{\circ} \mathrm{O}$. It also provides a test of the Eourier transform measurements published by Dr. Guelachvili near $1290 \mathrm{~cm}^{-1}$ (by means of the frequencies $01^{1} 1-01^{1} 0$ and $01^{1} 0-00^{0} 0$ ).

New heterodyne measurements have also been made on $\mathrm{N}_{2} \mathrm{O}$ in the region around $1100 \mathrm{~cm}^{-1}$ but due to a regrettable lack of coincidences, these measurements will not be very useful for improving the $\mathrm{N}_{2} \mathrm{O}$ calibration in the 1115 to $1340 \mathrm{~cm}^{-1}$ region. These measurements are primarily on hot bands and only include a few high $J$ levels of the P-branch.

2. New Measurements of Pressure Induced Frequency Shifts (A. G. Maki and A. S. Pine)

There are very few measurements of the pressure-induced irequency shifts of absorption features in the infrared. Because of this, it is difficult to estimate with confidence the magnitude of the frequency shift to be expected for a frequency calibration gas at a particular pressure. While making lineshape measurements on NO (described in section $C$ below), we were also able to measure the pressure-induced frequency shift of the lines. The shift of $-1.13 \pm 0.23 \mathrm{MHz} / \mathrm{kPz}$ of NO $(-0.15 \pm 0.03$ $\mathrm{MHz}$ (Torr) for the ${ }^{2} \Pi_{3 / 2}$ transitions and $1.05 \pm 0.23 \mathrm{MHz} / \mathrm{kPa}$ of NO $(-0.14 \pm 0.03 \mathrm{MHz} / \mathrm{Torr})$ for the ${ }^{2} \Pi_{1 / 2}$ transitions agrees in both sign and magnitude with the more poorly measured sinif of $C O$, a similar molecule 
with nearly the same mass and dipole moment. The shift is also similar to that measured for $\mathrm{N}_{2} \mathrm{O}$ although the shift of $\mathrm{N}_{2} \mathrm{O}$ was also rather poorly measured.

The present state of development of infrared frequency standards is shown in summary form in Figs. 2.1a and 2.1b, special attention belng given to the accuracies to which the various standards are known. Care must, however, be exercised in using these figures, for (1) in many cases there are gaps of 2 or $3 \mathrm{~cm}^{-1}$ between usable frequency standards and (2) it is difficult to find the appropriate papers in the literature since there is no complete compilation that would make it easy to find calibration data. A goal of this work is, of course, to make a compilation of calibration data available to everyone. In the meantime Art Maki is preparing a review article on frequency calibration standards to be published in the Journal of the optical Society. This review includes a bibliography of all papers presenting data that might be used for calibration.

It will be noticed in Fig. 2.1b that there are no adequate frequency standards from $2000 \mathrm{~cm}^{-1}$ to $4120 \mathrm{~cm}^{-1}$. In fact, from $2250 \mathrm{~cm}^{-1}$ to 4120 $\mathrm{cm}^{-1}$ there are no calibration standards (except a few isolated lines) with an absolute uncertainty of better than $\pm 50 \mathrm{MHz}$. Calibration in this important region obviously needs attention. At the moment there seems to be little interest in making heterodyne measurements in this region although we would certainly encourage such measurements. It may be possible to use $\mathrm{N}_{2} \mathrm{O}$ hot band transitions with the same upper state as the $\mathrm{N}_{2} \mathrm{O}$ bands between 4320 and $4755 \mathrm{~cm}^{-1}$ of the $\mathrm{N}_{2} \mathrm{O}$ bands from $3200 \mathrm{~cm}^{-1}$ to $4000 \mathrm{~cm}^{-1}$. Carefully calibrated Fourier transform measurements may be our principal hope for improving the available calibration data in the 2250 to $4120 \mathrm{~cm}^{-1}$ region.

It would be very valuable if some high rotational transitions of $\mathrm{HC} \&$ and $\mathrm{HF}$ and of vibrationally excited states of OCS and $\mathrm{H}_{2} \mathrm{O}$ could be measured using the new heterodyne techniques being developed for the far-infrared by Evenson and Jennings at NBS-Boulder. These measurements would naterially improve our ability to extend the infrared calibration tables to higher rotational levels and reduce our need to make heterodyne measurements on a wide range of ro-vibrational states.

In the coming year we plan to

1. Complete the compilation of calibration standards in the 480 $-700 \mathrm{~cm}^{-1}$ region.

2. Use Pine's difference-frequency laser spectrometer to measure the $10^{0},-000^{0}$ and $140_{0}-00^{\circ} 0$ bands of OCS between 2870 and $2980 \mathrm{~cm}^{-1}$ using the $\mathrm{CH}_{4} \mathrm{P}(7)$ line for calibration and Pine's carefully stabilized etalon to measure the small frequency differences involved. 


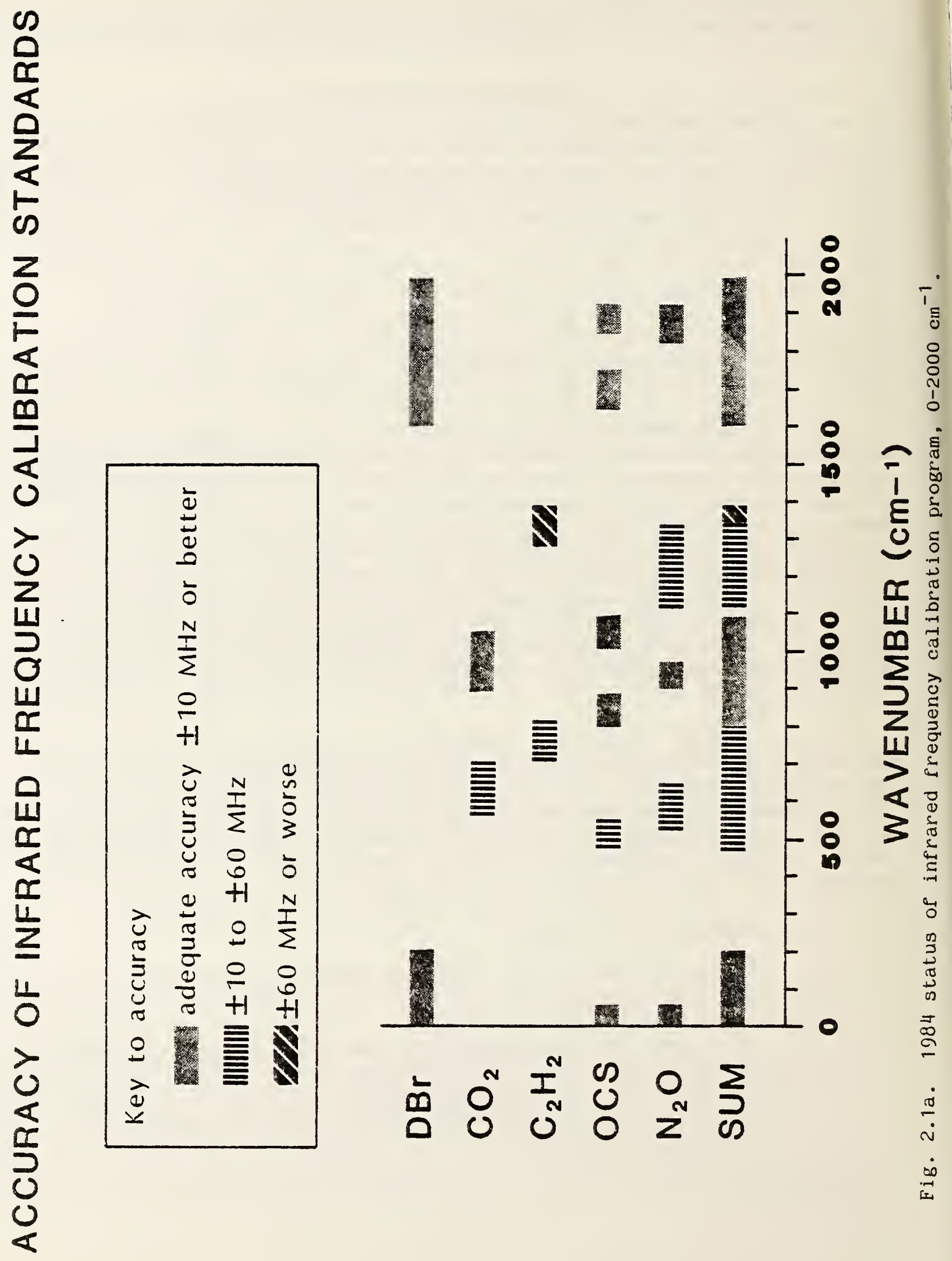



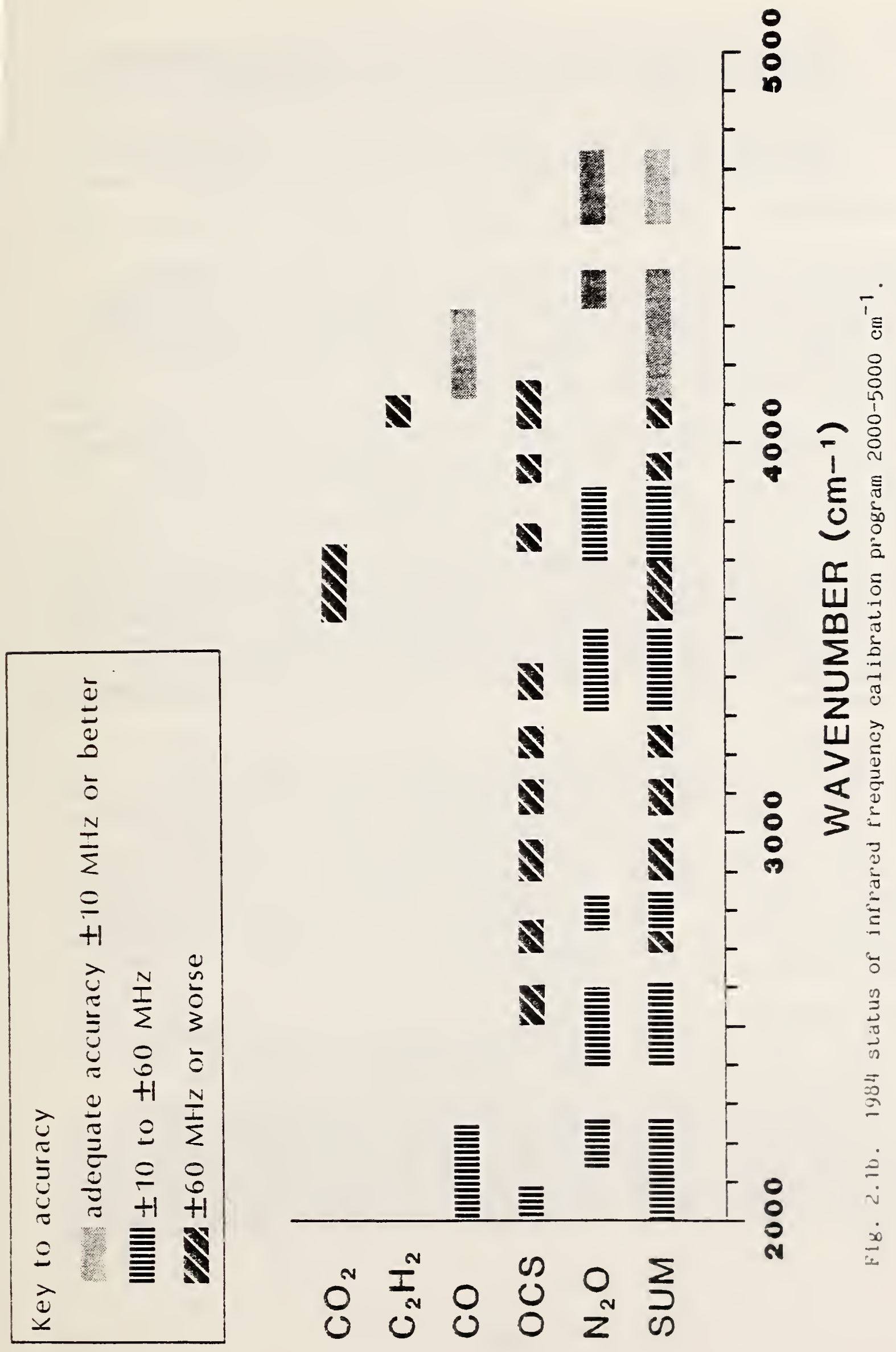
3. Add recently published microwave and laser-Stark measurements on OCS to our data base and recalculate some of the calibration tables for ocs.

4. Collaborate with NBS-Boulder on new heterodyne measurements of OCS and $\mathrm{N}_{2} \mathrm{O}$ including some far-infrared measurements.

\section{Spectroscopy in Environmental and Space Sciences}

Part of the Molecular Spectroscopy Division's task is to provide spectroscopic data and standards needed for the modeling of the earth's atmosphere, other planetary atmospheres, and interstellar matter. This work continues to receive some support from NASA's upper atmospheric research office because of our unique combination of spectroscopic expertise and measurement capabilities.

One of the missions of NASA is to understand the physics, chemistry, and transport properties of the earth's upper atmosphere in order to reliably predict the long term effects of anthropogenic perturbations of the atmosphere on the weather and on the protective ozone layer.

For years it has been known that the chemistry of the upper atmosphere is driven by a large array of interlocking chemical reactions. Because of the complex interdependencies of these reactions, the range of temperatures, and the uncertainties in circulation models, it has been diffictit to develop and test realistic models of the chemistry of the atmosphere, and particularly to extrapolate these models to hypothetical future atmospheric changes.

A key test of the validity of these models is to verify their predictions of the concentration of certain reaction intermediates. Another test is to verify predictions of diurnal variations, or the lack thereof, in the concentrations of certain trace species. For these tests, and to provide important input parameters for these models, it is essential to reliably measure the temporal and spatial distribution of many chemical constituents of the atmosphere. Many of the key atmospheric constituents are present in minute quantities, with mixing ratios of $10^{-9}$ or less, yet their role in the chemical chain is so important that NASA has given their measurement a high priority.

Spectroscopy is the obvious answer to NASA's remote measurements requirements. Many different types of spectroscopic devices have been designed and/or proposed for making atmospheric measurements from balloons, aircraft, and spacecraft, as well as from the ground. The design of these devices and experiments and the interpretation of data they produce require a knowledge of such spectroscopic properties as absorption frequencies, linewidths and shapes, and absorption intensities. In many cases the interfering effects of other atmospheric species must be understood in order to design a measurement technique and evaluate the results. 
The High Resolution Spectroscopy group is involved in assuring the integrity of atmospheric measurements in several ways. Our work in providing very accurate frequency calibration standards aids in comparisons between field and laboratory measurements as well as in inter-laboratory comparisons. Cur studies of lineshapes are providing a better understanding of the role collisions play in the shapes of absorption Ines in the upper atmosphere. Our work also provides the accurate spectroscopic data needed for the field measurements.

The Chemical Manufacturers Association (CMA) is also quite involved in supporting and coordinating atmospheric and laboratory measurements related to the ozone problem. This past year NBS hosted a workshop on atmospheric spectra sponsored by CMA and heavily attended by NASA researchers. This workshop concentrated on issues related to the measurement of ClO in the atmosphere, but many other topics related to molecular spectroscopy and both microwave and infrared measurements were also covered. In the coming year we expect to continue to work closely with CMA.

Such studies of atmospheric molecules also aid the Defense Department which needs to be able to predict the transmission properties of the atmosphere under various conditions of altitude, pathlength, temperature, etc. for various applications ranging from laser communication to infrared surveillance to various homing devices. We collaborate closely with Dr. L. Rothman of the Air Force Geophysical Laboratory (AFGL) who is involved in extending and improving the AFGL compilation of atmospheric absorption line parameters.

In the past, most of the work of the High Resolution Spectroscopy Group has involved the measurement and study of frequency data and, to a lesser extent, intensity data. Since Alan Pine's difference frequency laser spectrometer is uniquely suited to the study of Iineshapes, we have this year undertaken several projects for NASA to study the shapes of No, $\mathrm{HCl}$, and HF absorption lines. This work has resulted in new insights in the role of collisional narrowing for determining the lineshapes of these species in the atmosphere. The effects of pressure broadening have also been described with better accuracy than was hitherto available. In the case of the NO absorption lines a new phenomenon was observed giving rise to a pressure induced asymmetry in the linewidths of the closely spaced doublets observed in the $2 \Pi_{1 / 2}-2 \Pi_{1 / 2}$ transitions. In addition to these lineshape measurements we have also measured the intensities of the absorption lines with unprecedented accuracy.

We aiso have continued our efforts to provide and interpret inigh. resolution infrared spectra of $\mathrm{KNO}_{3}$ and $\mathrm{H}_{2} \mathrm{O}_{2}$. This past year our constants for the $v_{5}$ band of $\mathrm{HNO}_{3}$ were used by Dr. Colcman of the University of Denver to generate a linelist, including thousands of transitions of $\mathrm{HNO}_{3}$, which he is using to aid in the interpretation of his atmospheric spectra. 
1. Pressure Broadening, Lineshapes, and Intensity Measurements on NO

(A. G. Maki and A. S. Pine)

NO was chosen for lineshape measurements using Pine's difference frequency laser spectrometer because $N O$ is a key molecule in atmospheric chemistry, and yet it has a simple enough spectrum to allow us to measure the lineshape over a wide range of pressures.

This work has shown that the collisional narrowing coefficient for the 2-0 band is about $-1.7 \mathrm{MHz} / \mathrm{kPa}(-0.23 \mathrm{MHz} / \mathrm{Torr})$ for both $\mathrm{NO} / \mathrm{N}_{2}$ collisions and NO/NO collisions. This collisional narrowing coefficient is so small that it only results in a reduction of the effective Doppler width by about 9 percent at 50 Torr. Such a small reduction is almost negligible for most atmospheric applications.

One unexpected result of these measurements was the observation that the doublet structure of the lines of the ${ }^{2} \Pi_{1 / 2}-2 \pi_{1 / 2}$ transition acquires an asymmetry, at pressures of 50 Torr, that is not present at very low pressures. This asymmetry has been interpreted as a sign that the pressure broadening coefficient of one of the lines of the doublet pair is greater than the coefficient of the other line.

This work has also verified the observation of other workers that the pressure broadening coefficient of the ${ }^{2} \Pi_{3 / 2}$ state is greater than that of the $2 \pi_{1 / 2}$ state by about 6 percent. Fig. 2.2 shows the self-broadening coefficients measured for the 2-0 band of No.

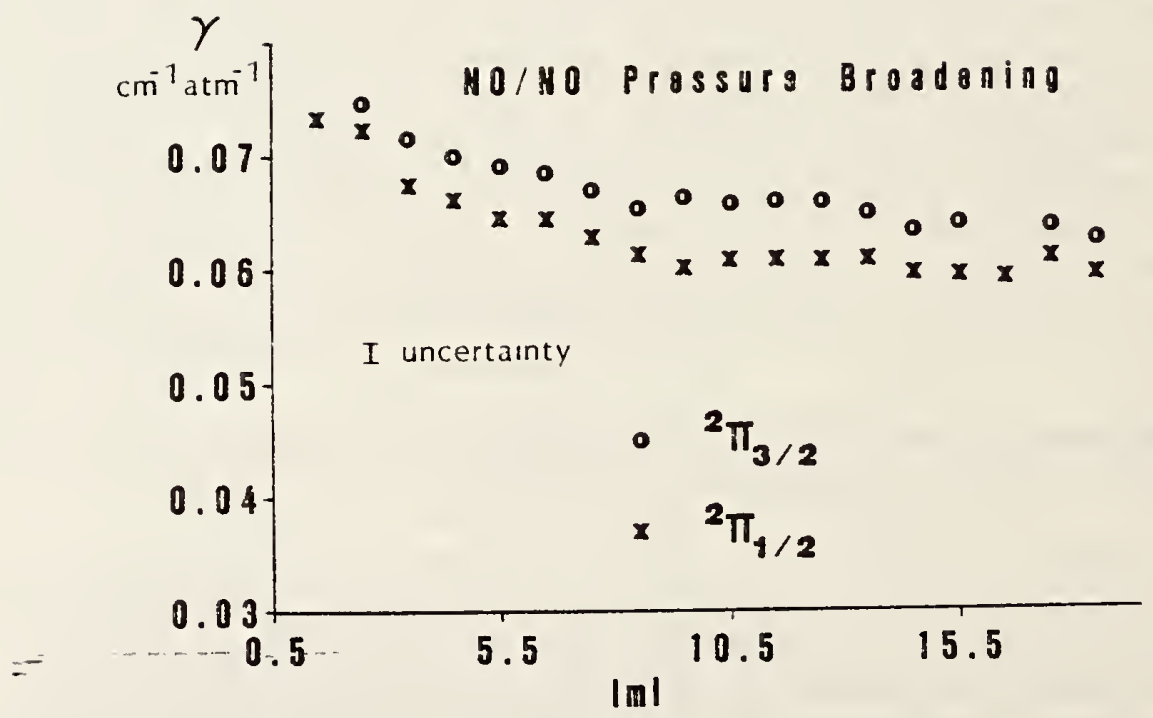

Fig. 2.2. Self-broadening coefficients for the 2-0 band of No at $296 \mathrm{~K}$. 
The present measurements give the best line intensity values yet obtained for NO. In addition to providing an improved value for the transition moment, these measurements give for the first time an accurate experimental value for the Herman-Wallis effect. This Herman-wallis term is in agreement with only one set of relative signs for the coefficients of the dipole-moment expansion of NO, and the agreement between the observed and calculated values is very good. $[\alpha$ (obs) $=0.00357 \pm 0.00014$; $\alpha($ calc $)=0.00361]$. This research has been supported in part by NASA-Langley Research Center and NASA-Headquarters.

2. Spectral Intensities in the Fundamental Bands of $\mathrm{HF}$ and $\mathrm{HCl}$ (A. S. Pine, A. Fried (CAC) and J.W. Elkins (CAC))

In coilaboration with Drs. A. Fried and J. W. Elkins of the Center for Analytical Chemistry the transition intensities of the fundamental bands of natural isotopic $\mathrm{HF}$ and $\mathrm{HC} 2$ have been measured with Doppler-limited resolution using a tunable difference-frequency laser spectrometer. Measurements were carried out in support of the NASA Upper Atmosphere Project HALOE to monitor ozone depletion caused by reactions with halogen-containing molecules. Precise values for the integrated band intensities, $S_{v}$, the vibrational transition moments, $\left\langle\mu_{v}\right\rangle$, and the Herman-Wallis factors, $\left(1+\mathrm{C}_{2} \mathrm{M}+\mathrm{C}_{2} \mathrm{M}^{2}\right)$, have been determined. Particular care was taken with sample harding of these reactive vapors to ensure minimum impurities and accurate pressure measurements. Detailed lineshapes were examined for deviations from predicted Doppler-Gaussian profiles induced by unresolved hyperfine splittings and from instrumental distortions due to scan rate, baseline uncertainties, system nonlinearities, noise and laser jitter. This research has been supported in part by NASA-Headquarters.

3. New Developments in the Study of Nitric Acid $\left(\mathrm{HNO}_{3}\right)$ (A. G. Maki)

Continuing a project briefly described last year, we have this year measured and assigned the spectrum of the $2 v_{g}$ band of nitric acid $\left(\mathrm{HNO}_{3}\right.$ ) near $890 \mathrm{~cm}^{-1}$. This band is in rescnance with the $y_{5}$ band, which was studied last year, and an understanding of the spectrum of nitric acid requires that we understand this interaction. The analysis of the $\nu_{5}$ band was very important for assigning the transitions of the $2 v_{g}$ band because it told us which transitions of $2 v_{g}$ would be displased due to the interaction with $v_{5}$ and it also greatly simplified the spectrum where the tio bands overlap.

We now have a good set of band constants that describes quite well the nitric acid spectrum from $850 \mathrm{~cm}^{-1}$ to $925 \mathrm{~cm}^{-1}$ with the exception of the most strongiy perturted transitions. In the coming year we plan to use our new perturbed asymmetric rotor fitting program to obtain an analysis that will even incluce the badly perturbed iransitions. 
This work is currently being carried on in collaboration with Dr. Aaron Goldman of the University of Denver. We had hoped to obtain Fourier transform spectra from Oulu, Finland, but the spectra were not very good due to the reaction of the nitric acid with the absorption cell windows. Dr. Goldman was able to provide us with spectra taken with a resolution of $0.004 \mathrm{~cm}^{-1}$ which was adequate to resolve many of the transitions.

In addition to using the Fourier transform data, we have been able to measure many portions of the spectrum with our tunable diode laser spectrometer. Of special importance was the measurement and analysis of the very crowded $Q$-branch regions for both the $v_{5}$ and $2 v_{9}$ bands. The spectrum of a part of the $v_{5} Q$-branch is shown in Fig. 2.3 .

\section{$\mathrm{HNO}_{3} \quad V_{5}$ Q-BRANCH}

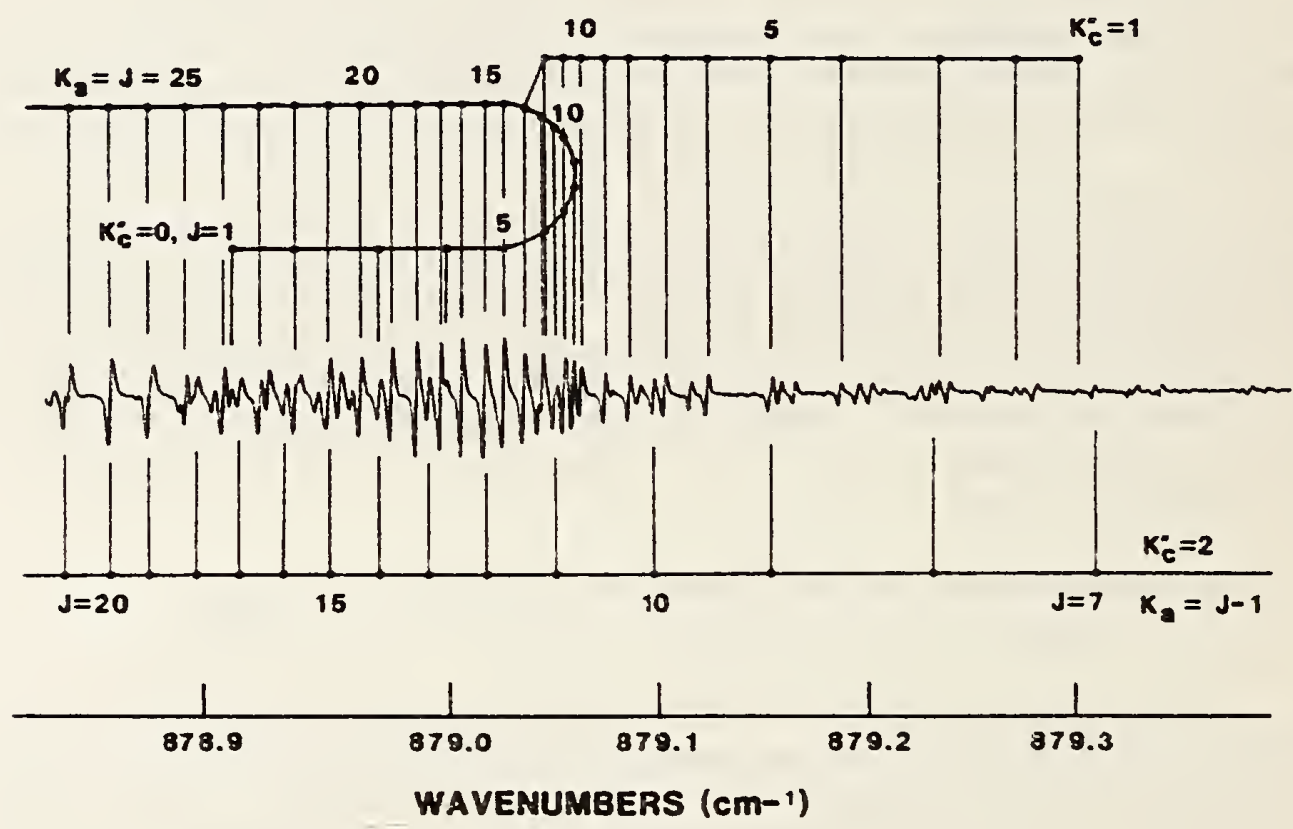

Figure 2.3. The first-derivative spectrum of the Q-branch region of $v_{5}$ of $\mathrm{HNO}_{3}$. The $\mathrm{K}_{\mathrm{a}}=\mathrm{J}$ transitions are identified above the spectrum and the $\mathrm{K}_{\mathrm{a}}=j-1$ transitions for $\mathrm{K}_{\mathrm{c}}=2$ are identified below the spectrum. All identified transitions are A-type Q-branch transitions.

One result of this work that is of scientific interest, although it has little interest for atmospheric studies, was the discovery that some of the $2 v_{9}$ transitions are coublets with a splitting of about $50 \mathrm{MHz}$. The $v_{g}$ vibration is an $\mathrm{OH}$ torsional mode and the splitting of the $2 \mathrm{vg}_{\mathrm{g}}$ transitions is due to a torsional tunneling of the hydrogen atom from one side of the planar molecule to the equivalent opposite side. The 
splitting gives us a rough idea of the height of the torsional barrier, but the spectra show that there is a strong variation of the cunneling splitting with rotational state.

The best way to study the torsional barrier would be to look at the $v_{9}$ band at $450 \mathrm{~cm}^{-1}$ and particularly at the hot band $3 v_{9}-2 v_{9}$ which should be easy to analyze since we already have the rotational constants for the $2 v_{9}$ state and we know which levels are perturbed by the interactlon with $v_{5}$. Such a study of the $v_{9}$ band would also help in the analysis of the hot band connected with the $v_{5}$ and $2 v_{9}$ bands. These hot band transitions are obviously contributing to the atmospheric spectra and Goldman has already attempted to take them into account. This work has been supported in part by NASA-Langley Research Center.

\section{Analysis of the Spectrum of $\mathrm{H}_{2} \mathrm{O}_{2}$}

(W. B. Olson)

Considerable progress has been made in the assignment of hydrogen peroxide lines in the region of the bending combination around $2650 \mathrm{~cm}^{-1}$. About forty subbands have been assigned in the bending combination, and about seventeen have been assigned in each of the two overtone bands.

Torsional separations have been determined in all three bands to about $0.001 \mathrm{~cm}^{-1}$. The center of the strongest band, the a-plus b-type hybrid band $v_{2}+v_{6}$, is at $2649.014 \mathrm{~cm}^{-1}$ and the torsional separation is $1 \mathrm{i} .712 \mathrm{~cm}^{-1}$, only slightly above the ground state value of $11.437 \mathrm{~cm}^{-1}$. For $2 v_{6}$, a c-type band, with different torsional selection rules irom the combination band mentioned above, the band origin is at $2494.815 \mathrm{~cm}^{-1}$, but the vibrational origin is actually higher by $11.437 \mathrm{~cm}^{-1}$, at 2506.252 $\mathrm{cm}^{-1}$. The upper state torsional splitting is very much larger than that in the ground state, being $33.029 \mathrm{~cm}^{-1}$. For $2 v_{2}$, also a c-type band, the vibrational origin is again $11.437 \mathrm{~cm}^{-1}$ above the ooserved orizin of $2754.555 \mathrm{~cm}^{-1}$, but the torsional splitting is here only $3.533 \mathrm{~cm}^{-1}$.

Ne now have available an asymmetric rotor fitting progran, written by Art Maki which will fit two vibrational states with an interaction between them, by least squares. We will pool data with Prof. R. Hunt, of Florida State iniversity in order to it the grolind state perturbation. This work was supported in part by NASA-Goddard Space Flight Center.

D. Spectroscopy of Hydrogen-Bonded Systems and Other Clusters

1. Vibrational Predissociation in the HF and DF Dimers (A. S. Pine and W. J. Lafferty)

We previously reported a mode-specific viorational predissociation rate in the HE dimer based on different ro-vibrational transition linewidths observed for the "Iree-H" stretching band, $v_{1}$, and the "bound-H" stretching band, $v_{2}$, recorded with a high-resolution difference-frequency laser spectrometer. Predissociation occurs because the excited $\mathrm{H}-\mathrm{F}$ stretching bands are metastable since they have roughly tilce 
the frequency of the hydrogen bonding energy of the complex. Linewidths in excess of the known Dcppler and pressure-broadening contributions by about $200 \mathrm{MHz}$ (HWHM) were observed for $v_{2}$ lines, corresponding to a lifetime of about $0.8 \mathrm{nsec}$. No excess widths were resolvable for $v_{1}$ lines. Recently, DeLeon and Muenter, using subDoppler molecular beam techniques, have established a lower limit of $>30$ nsec for the "free-H" predissociation lifetime. This mode specificity indicates that direct coupling to the hydrogen-bond stretching coordinate is the dominant predissociation channel rather than a rapid intramolecular energy randomization as invoked by statistical models.

We have now examined the high resolution spectrum of the DF dimer for clues to the predissociation mechanism provided by the isotopic variation. Here the binding energy of the complex and the total mass of the fragments are changed only marginaliy, but the $D-F$ stretching frequencies are reduced by $-\sqrt{ } 2$ and the rotational constants of the fragments by -2 . In the spectrum of the DF dimer, we observe no excess broadening for either the $v_{1}$ band "free-D" stretch or the $v_{2}$ band "bound-D" stretch. This result is in clear distinction to "momentum gap" predissociation models based on exponentially increased overlap with continuum translational wavefunctions for the reduced energy above dissociation for the D-F stretches. These models predict (DF) 2 predissociation rates, and hence linewidths $-10^{3}$ greater than for ( $\left.\mathrm{HF}\right)_{2}$. At present we have neither a quantitative nor a qualitative explanation for these results, but they are clearly important for understanding mode-selective chemistry and intramolecular energy transfer.

2. High Resolution Spectrum of the HCl Dimer

(N. Ohashi and A. S. Pine)

Rotational structure in the spectrum of the $\mathrm{H}-\mathrm{Cl}$ stretching bands of the HC2 dimer has been fully resolved using a tunable difference-frequency laser. The spectrum of a natural isotopic sampie was recorded under thermal equilibrium conditions near the condensation point $(\mathrm{T}-130 \mathrm{~K}$ ) of $\mathrm{HCl}$ at low pressures (3-4 Torr) and long pathlengths ( $64-80$ $\mathrm{m})$. A portion of the spectrum in the null gap of the monomer is shown in Figure 2.4. Rotational assignments have been made for $K^{\prime \prime}=0,1$ and 2 subbands of a perpendicular-type band centered near $2880 \mathrm{~cm}^{-1}$ and a parallel-type band near $2857 \mathrm{~cm}^{-1}$ sharing a common vibrationai ground state. For the ground state of the $\mathrm{H}^{35} \mathrm{Cl}-\mathrm{H}^{35} \mathrm{Cl}$ dimer, the $\mathrm{K}^{\prime \prime}=0$ rotational constants, $B=1944.29(7) \mathrm{MHz}$, and the $K "=1$ asymmetry splitting, $B-C=19.5(3) \mathrm{MHz}$, yield a zero-point center-of-mass separation of the two monomer constituents of $3.797(1)$ A. Another difference band for the $35 \mathrm{Cl}$ isctope has also been observed as well as a similar set of bands for the mixed $\mathrm{H}^{35} \mathrm{Cl}-\mathrm{H}^{37} \mathrm{C} 2$ species. The spectra suggest an extremely rapid tunneling motion between equivalent forms of nearly crthogonally oriented monomer units. The single bandhead spike shown on the ${ }^{r} Q_{0}$ subbranch contour for the mixed dimer species in Eigure 2.4 is a manifestation of tunneling-averaging of the two inequivalent equilibrium isotopic conformations expected. 


\section{$\mathrm{HCl}$ DIMER}

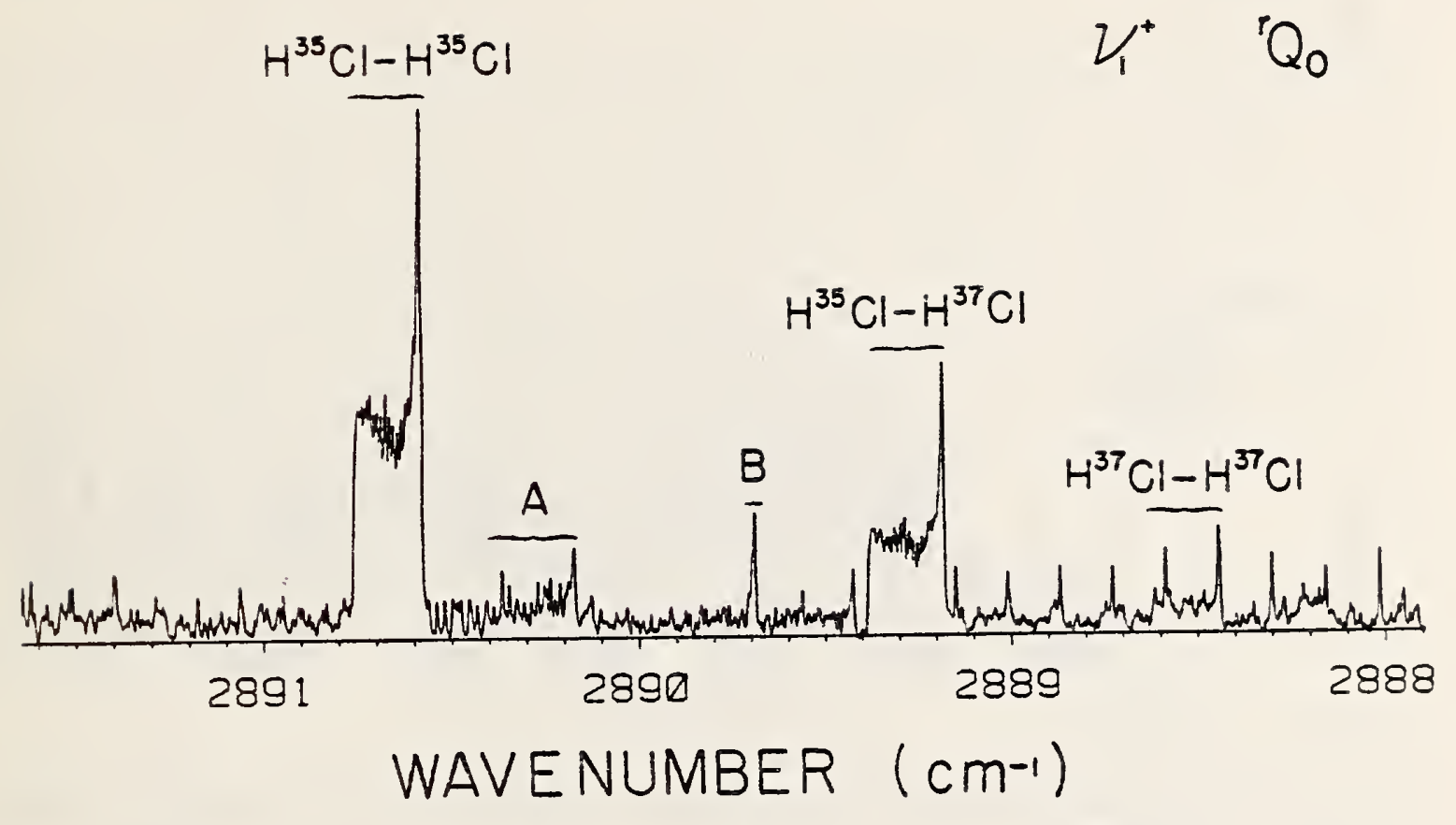

Fig. 2.4. Transitions in the Q-branch region of the HCl dimer.

3. Resolved Infrared Structure in the Ar-HCl van der Waals Complex (B. J. Howard and A. S. Pine)

We have resolved the rotational structure associated with the $\mathrm{H}-\mathrm{Cl}$ stretching vibration of the Ar-HCl van der waals ccmplex using a difference-Prequency laser spectrometer. A portion of the spectrum exhibiting a regular series of lines attributed to the $\mathrm{R}$-branch of the fundamental band is shown in Figure 2.5. The spacing between the lines yields information about the rotational constant, $B$, and the size of the complex is in excellent agreement with earlier molecular-beam electric-resonance studies of the ground state. The convergence of the lines at higher wavenumber yields the vibrational dependence of the $B$ value implying a dominant repulsive wall for the van der Waals potential. The sharp cut of $f$ of intensity above $2888.7 \mathrm{~cm}^{-1}$ is a unique feature indicative cf rotational dissociation as the rotational energy exceeds the binding energy and the centrifugal barrier. The sharp, nearly Doppler-limited, linewidths are evidence for a relatively slow vibrational predissociation rate. Nork is proceeding to assign hot bands and combination bands in this region which should reveal direct information about the low frequency van der wals bond bending and stretching motions. 


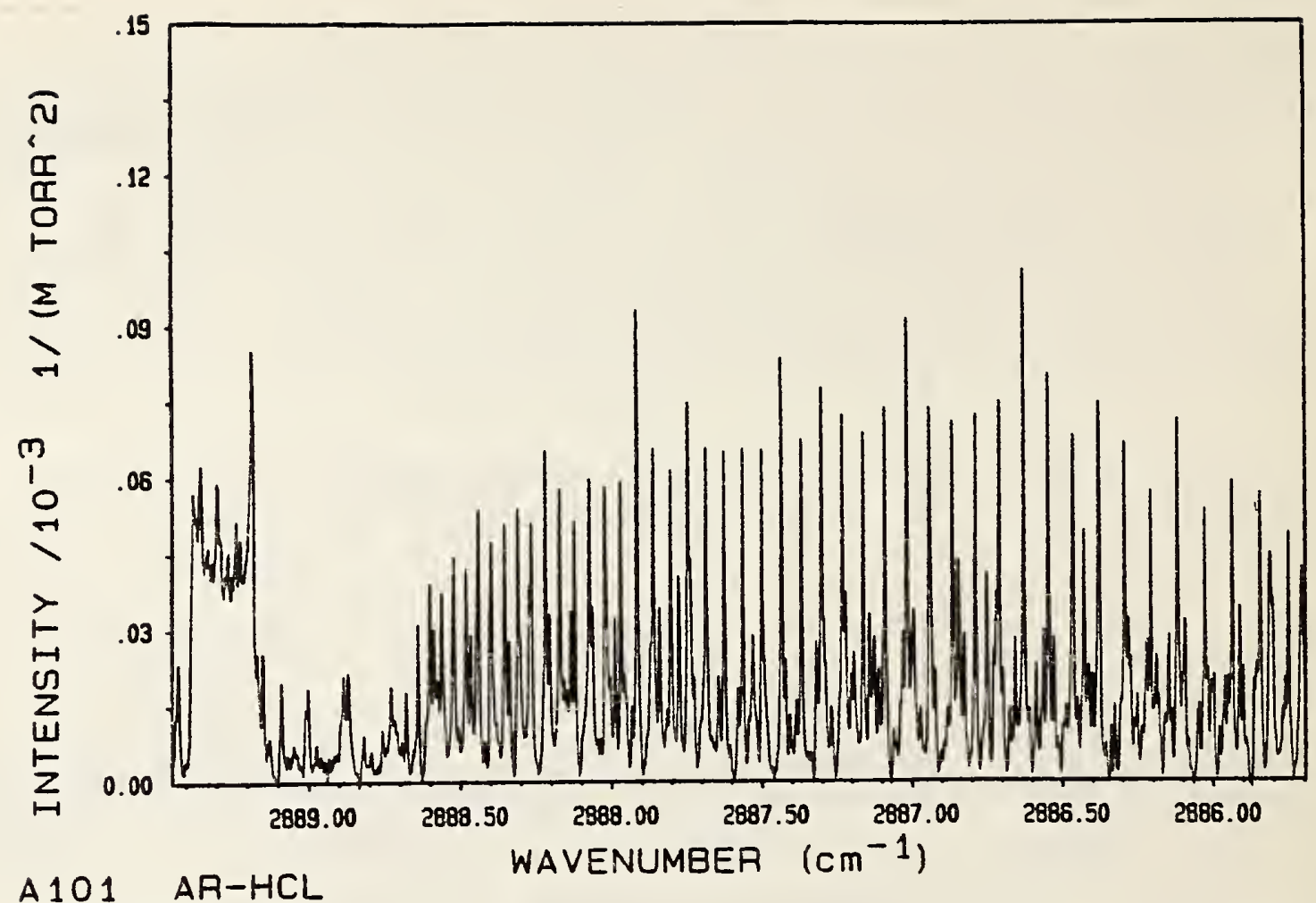

Fig. 2.5. Portion of the infrared spectrum of the Ar-HCl van der Waals complex.

4. MetaI Clusters

(A. Weber and M. Raab)

Using the apparatus completed during the previous year experiments were performed to obtain rotationally resolved spectra of sodium and lithium trimers produced in a supersonic expansion. A two step photoionization method was used employing a tunable single frequency $c w$ dye laser ( 1 ine width less than $1 \mathrm{MHz}$ ) for excitation and a $\mathrm{cw}$ fixed frequency ionizing laser. Signal detection was accomplished by a quadrupole mass spectrometer iuned to the mass peak of tine appropriate trimer. Though strong trimer signals were observed no resolved rotational structure was seen. It is not established whether this failure results from bound-free transitions, pre-dissociation, or congestion of rotational transitiors. Further work on other systems is planned.

E. Fourier Transform Spectroscopy

i. Fourier Transform Infrared Spectroscopy

(A. Weber, W. B. Olson, and W. I. Lafferty)

The BOMEM DA3.002 high resolution Fourier transform spectrometer delivered last year has, since the last report, undergone extensive tests. As presently equipped the instrument spans the spectrum between 10 and 
$8000 \mathrm{~cm}^{-1}$ and additional optics for use in the visible to 4000 A have been ordered. With a maximum optical path difference of $250 \mathrm{~cm}$ the maximum unapodized resolution is $0.0025 \mathrm{~cm}^{-1}$.

In addition to performing the usual tests with gases such as CO, OCS, $\mathrm{HCl}$ and others, all of which have simple and uncrowded spectra, we decided to do additional performance testing with cyanogen $\left(\mathrm{C}_{2} \mathrm{~N}_{2}\right)$. This is because cyanogen has a fairly dense spectrum, which more closely approximates the line density of spectra that are to be studied, and also because the spectrum can be fully understood on the basis of the Hamiltonian of a linear molecule (for a report on this work see section E.4). Tests for the relative and absolute frequency accuracy showed that in the region of $2100 \mathrm{~cm}^{-1}$ the measured frequencies are of fset from the accepted values by $+0.003 \mathrm{~cm}^{-1}$ on the average, while the precision of the measurements is about 10 times better. For high accuracy work the frequency scale must be calibrated using independently determined molecular absorption irequencies.

In addition to the work on cyanogen mentioned above, the instrument has also been used to generate spectra of hydrazine and deuterosilane. Descriptions of these activities are given in sections E.5 and E.6.

The range of use of the instrument has been enlarged through the design and construction of special transfer optical systems to permit its employ in matrix isolation studies [see section 3.E2], and for use with a 20m multipass White cell. An additional transfer optical system is being designed for use with a coolable $100 \mathrm{~m}$ multipass White cell. An independent study of the optical system of a White cell for use with Eourier transform spectrometers was undertaken. These activities are reported in sections $\mathrm{E} 2$ and $E 3$.

2. Transfer Optics Systems

(W. B. Olson)

Two transfer optics systems have been designed and constructed in order to utilize the BOMEM ETS for specialized experiment studies. The first of these is for interfacing to Marilyn jacox's apparatus for matrix isolation reflection spectroscopy (See Section 3. 22 ), and the second for coupling to a commercial Iong path multiple reflection absorption cell. For both optical transfer systems, the maximum possible throughput was determined from unavoidable geometrical and optical constraints, and ray transfer matrices were used to design the system to obtain the maximum throughput and maximum spectral wavelength coverage. In particular, we are able to work in the very far infrared region with the limiting throughput being that of the BOMEM instrument, and not that of the external apparatus.

For the interface to Dr. Jacox's apparatus the system is of a modified Czerny-Turner design to minimize aberrations. Furthermore the cold reflection mirror of the matrix isoiation apparatus is a field mirror in order to conserve all of the available lizht. While it is true that with longer wavelength optical perfection requirements are relaxed, one 
cannot be too sloppy about design if one wishes to optimize performance. An aberrated image requires a larger than necessary detector area to collect all of the radiation, and this larger detector area leads to an increase in the noise level, resulting in a loss of information. Also, for a system that is to have wide spectral coverage, the optical perfection should be based upon the shortest wavelength to be observed.

For the second system, the transfer optics to the multiple reflection absorption cell, we have used relatively long focal length imaging mirrors, which require several flat mirrors for beam folding. These flat mirrors lead to some reflection loss, but the long focal length and low off-axis angles at the reimaging mirrors lead to a good image at all foci, and to a near ideal image diameter. One discovery about the utility of ray transfer matrix design was made during the design process.

In order to conserve all available light the windows of a multiple reflection absorption cell are usually field lenses. It is usual to assume that these lenses are at the front focal plane of the multiple pass cell. In fact, they are not. We found that with a short base path cell one could get little or even no effect by using field lenses calculated for the focal plane position at the position actually required by the real geometry, i.e., at about an inch in front of the focal plane. It was found that a proper field lens focal length can be found for use at the actual required physical position, that leads to the same optical transfer function at the front focal plane of the multiple pass cell, and at the back mirrors of it, as for the ideal case.

3. White Cell Design

(i. B. Olson)

We have put together some new ideas on multipassing, throughput of a multipass cell, and astigmatism to give a new system of design for multipass absorption cells for use with circular or square apertures. This treatment provides exact criteria for the geometry required to minimize the volume of an absorption cell and astigmatism of the absorption cell (the major optical aberration limiting performance), given cnly initial conditions required by physics. A paper giving the derivation and results of this investigation has been published in Applied Optics.

Calculations show that it should be possible to reduce the volume by a factor of two to four below most "good" existing multipass cells and reduce the effect of astigmatism at the same time. A conservative estimate is that one should be able to make a multipass cell with six rows and seven columns of images on the front mirror with low reflectivity and astigmatism losses. This cell would have 78 total transits. Somewnat more optimistic would be the possibility of eight rows and nine columns of images on the front mirror for 136 transits. With 22.5 meter base path one could match the throughput of the BOMEM FTS instrument (which is relatively large) down to $55 \mathrm{~cm}^{-1}$ (a worst case) with a calculated cell transmission of 0.25 , which is not unreasonably low. 
Work was started on the analysis of the spectrum of cyanogen, $\mathrm{C}_{2} \mathrm{~N}_{2}$, as part of our testing program of the BOMEM Fourier transform spectrometer (see section E.1). Since previous work done on cyanogen was done at a resolution inferior than that available with the BOMEM instrument a new analysis of the infrared spectrum of $\mathrm{C}_{2} \mathrm{~N}_{2}$ was undertaken. Of the five fundamental vibrations only two are infrared active, the $v_{3}$ anti-symmetric stretching vibration (parallel band) and the doubly degenerate $v_{5}$ anti-symmetric bending vibration (perpendicular band). Spectra were obtained with gas pressures of 1 and 5 torr and an absorbing path length of $20 \mathrm{~cm}$. Figures 2.6 and 2.7 show the $v_{3}$-parallel band resulting from the coaddition of 25 interferograms recorded in a total time of 125 minutes. Band analyses have been performed for $v_{3}$ and the associated identified hot bands. Twenty coadded interferograms of the low frequency perpendicular band $v_{5}$ at $235 \mathrm{~cm}^{-1}$ were also recorded at full resolution in $81 / 2$ hrs. Since this band falls in a region where the black body emission from the globar source is low and where the bolometer detector is less sensitive, much more time was required for the recording of this band than was needed for $v_{3}$. Both band systems have been analyzed and preliminary results have been reported. Further work is in progress.

5. A Study of Inversion and Torsional Splittings in the Low Frequency Torsional Band of Hydrazine (N. Ohashi, W. B. Olson, and W. J. Lafferty)

From a spectroscopic viewpoint hydrazine $\left(\mathrm{NH}_{2}-\mathrm{NH}_{2}\right)$ is a fascinating molecule. It has two large amplitude vibrations. One, a $\mathrm{NH}_{2}$ wagging motion, is analogous to the umbrella motion in $\mathrm{NH}_{3}$ but with a sufficiently small barrier to inversion to produce a large splitting in the ground state energy levels. The other is a torsional vibration, with a small enough barrier to internal rotation to permit tunneling through a trans barrier; a small but observable splitting from this source has also been observed in the pure rotational spectrum. Only very recently has the microwave spectrum been completely understood (1) with the aid of a theoretical formalism based on double group theory developed in this laboratory by Hougen (2).

$\mathrm{cm}^{-1}$, has spectrum of the torsional band of $\mathrm{N}_{2} \mathrm{H}_{4}$, which is centered at 376 $\mathrm{cm}^{-1}$, has been recorded on the NBS BCMEM Fourier transform spectrometer. This band was chosen for study for a number of reasons. The torsional splitting in this band is much larger than in the ground state, and ine determination of this parameter will lead to a more accurate estimate of the barrier to internal rotation. In addition, this band is the Iowest frequency vibration in $\mathrm{N}_{2} \mathrm{H}_{4}$ and is suficiently isolated from other vibrations so that the assignment will not be complicated by the presence of accidental resonances. Also, this band fortunately is a B-type band 


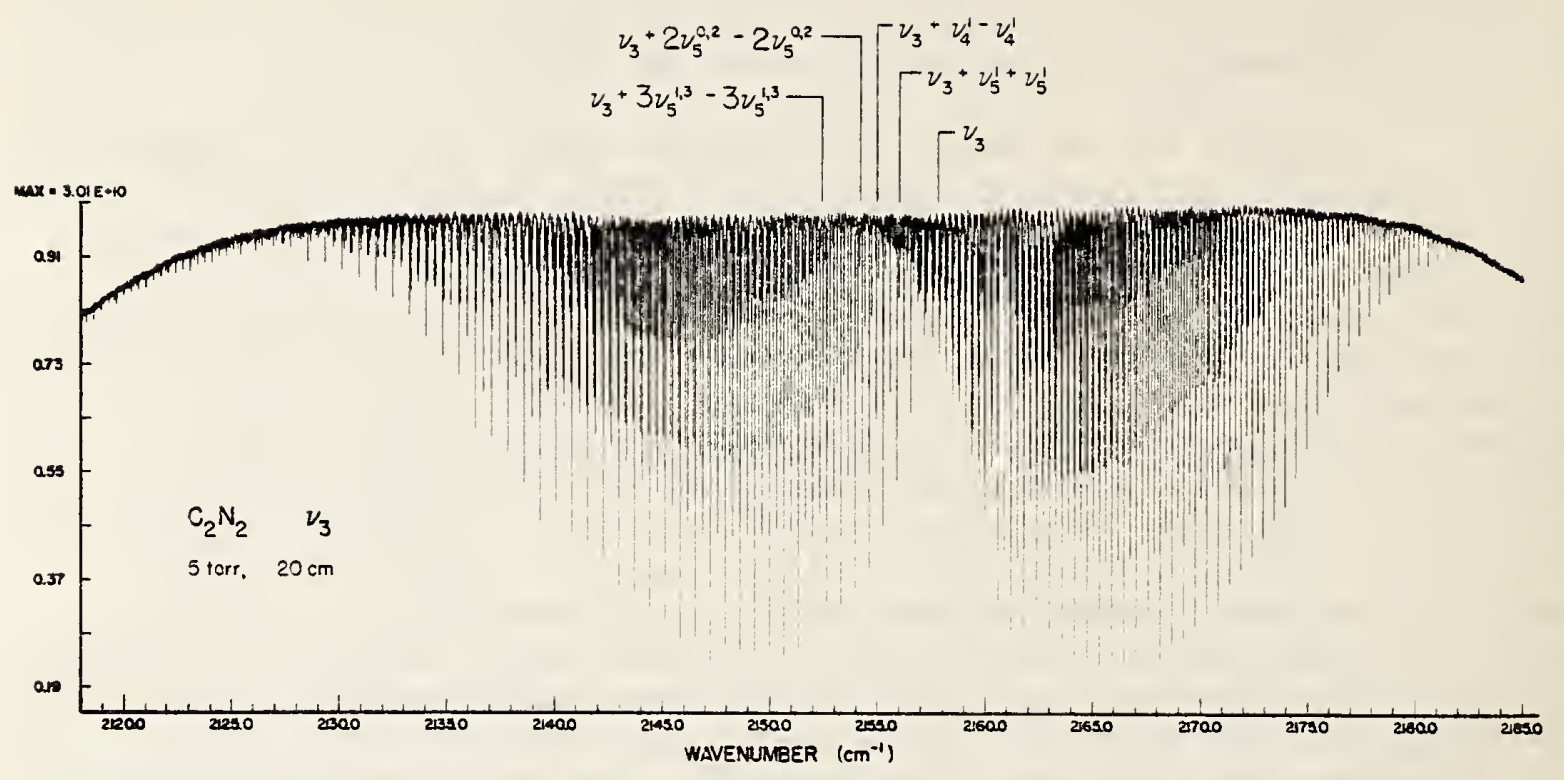

Fig. 2.6. Overview of the $v_{3}$-band of $\mathrm{C}_{2} \mathrm{~N}_{2}$ recorded at a resolution of $0.004 \mathrm{~cm}^{-1}$.

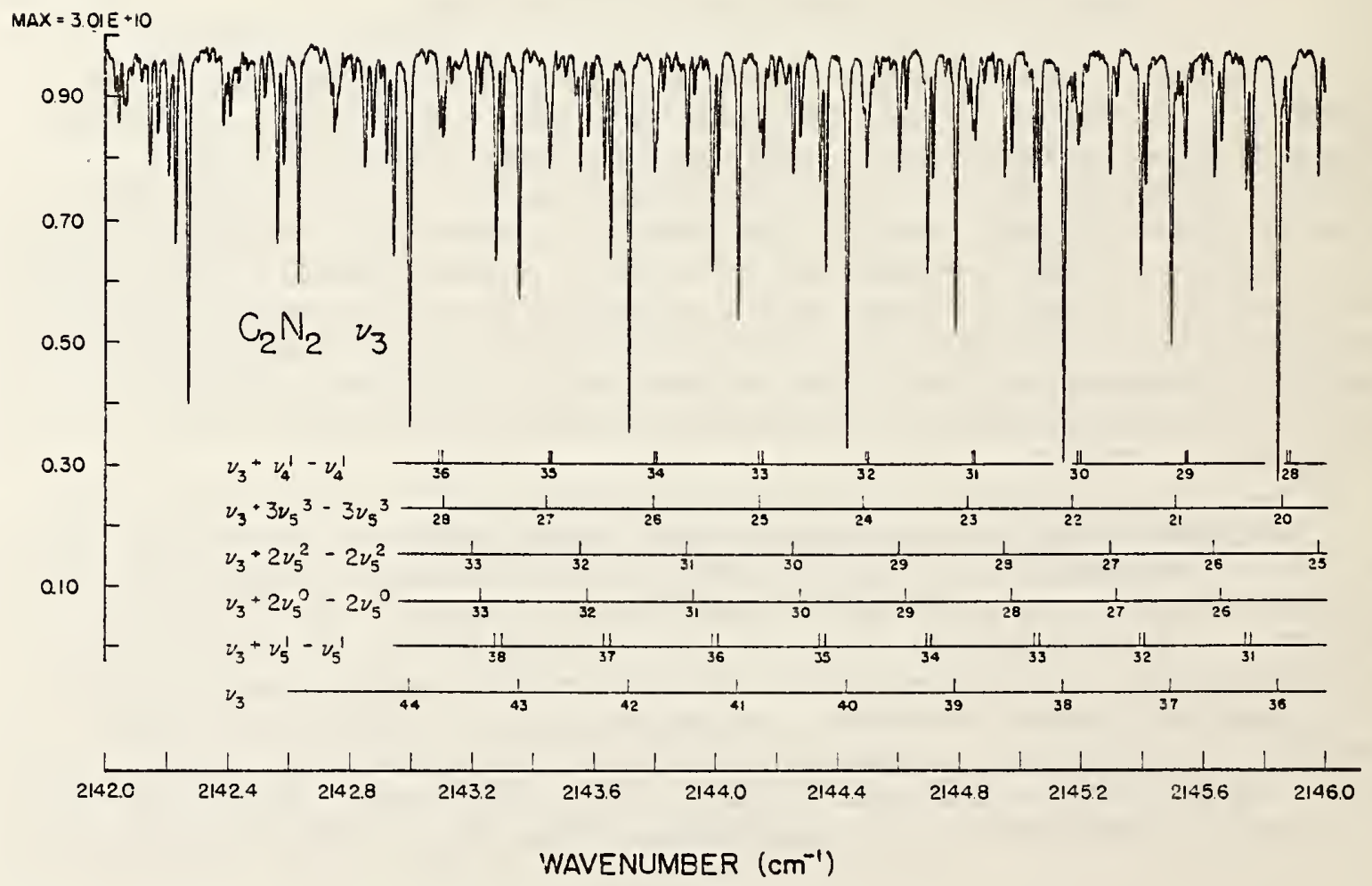

Fig. 2.7. Detail of $v_{3}$-band of $\mathrm{C}_{2} \mathrm{~N}_{2}$ with partial assignment. 
which very closely resembles a perpendicular band of a symmetric top molecule. Such bands spread over a large frequency range and ine overlapping is reduced.

Line assignment has been started and, at this point, a number of

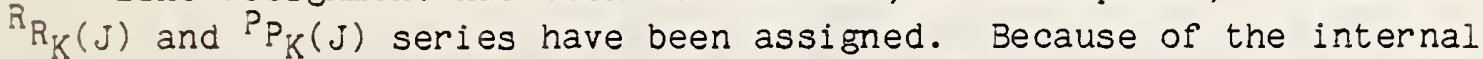
motions of $\mathrm{N}_{2} \mathrm{H}_{4}$, these transitions appear as well-resolved quartets or sextets, depending on whether $K^{\prime \prime}$ is even or odd. Except for the $K^{\prime \prime}=2$ lines, which are broadened, asymmetric rotor splitting is not observed.

Although the results are very preliminary, approximate values of both the torsional and inversion splittings have been derived. The torsional splitting is about $2050 \mathrm{MHz}$ compared to $5.796 \mathrm{MHz}$ in the ground state. The inversion splitting shows a surprising vibrational dependence, being roughly $7580 \mathrm{MHz}$, about half that of the ground state splitting (16040 $\mathrm{MHz}$ ).

(1) Tsunekawa, T. Kojima, and J. T. Hougen, J. Mol. Spectrosc. 95, 133 ( 1982$)$.

(2) J. T. Hougen, J. Mol. Spectrosc. 89, 296 (1981).

6. Fourier Transform IR Spectra of Mono- and Tri-Deuterosilane (R. W. Lovejoy and W. B. Olson)

Previously reported work $(1,2)$ on $\mathrm{SiH}_{3} \mathrm{D}$ and $\mathrm{SiHD}_{3}$ was limited to those vibration-rotation bands above $2000 \mathrm{~cm}^{-3}$ because of spectrometer considerations. With the availability of the BOMEM spectrometer, the entire infrared spectrum of these molecules can now be studied at 10 times the previously available resolution. Thus far a spectrum of the $v_{3}, v_{5}$, and $v_{6}$ bands of $\mathrm{SiH}_{3} D$ has been obtained at $0.005 \mathrm{~cm}^{-1}$ resolution by addi.ng 64 interferograms: The spectrum of $v_{2}$ has also been obtained at a resolution of $0.004 \mathrm{~cm}^{-1}$. For all these spectra the pressure was 5 Torr and the pathlength $20 \mathrm{~cm}$. These four bands, in addition to the two Si-H stretching fundamentals studied previously, complete the spectra of all of the fundamentals of this molecule.

At present most subbands of $v_{\sigma}$ have been assigned. A preliminary fit places the band origin at $784.323 \mathrm{~cm}^{-1}$. In the parallel component of the overtone $2 v_{\sigma}$ at $1557.258 \mathrm{~cm}^{1}$, ten subbands have been assigned, jut the perpendicular component has not yet been identified. The $v_{3}$ and $v_{5}$ bands are strongly Coriolis coupled and will be difficult to assign at high $J$, however the band origin of the former has been determined to be 912.993 $\mathrm{cm}^{-1}$. Six subbands of $\nu_{5}$ have been assigned. These assessments only permit an estimate of $\mathrm{R}_{0}^{5}$ at about $952.7 \mathrm{~cm}^{-1}$. The origin of $v_{2}$, the $\mathrm{Si-}$ stretching frequency, is at $1593.967 \mathrm{~cm}^{-1}$.

(1) Olson, W. B. and Lovejoy, R.W., J. Mo. Spectrosc. 66, 314-330 (1977). 
(2) Fromer, C., Lovejoy, R. W., Sams, R. L., and Olson, W.B., J. Mol. Spectrose. 89, 261-267 (1981).

7. Fourier Transform Raman Spectroscopy of Gases (A. Weber and D. E. Jennings)

Spontaneous, incoherent Raman spectroscopy of gases at high resolution has been successfully performed in the past using specially built grating spectrographs or spectrometers. The techniques based on spectrographs have been fully developed $(1,2)$ with the best resolution attained being in the neighborhood of $0.05 \mathrm{~cm}^{-1}$. Very much higher resolution $\left(-0.001 \mathrm{~cm}^{-1}\right)$ is achieved using coherent Raman scattering techniques among which the CARS (Coherent Antistokes Raman Scattering) and SRGS (Stimulated Raman Gain Spectroscopy) methods are the best known. Since the limiting resolution is in principle governed by the Doppler effect the use of high resolution Fourier transform spectrometers suggests an alternative to the coherent laser techniques for obtaining high resolution Raman spectra. Since there has been no report of the successful use of Fourier transform (FT) spectrometers in Raman spectroscopy a pilot study was undertaken in collaboration with $\mathrm{J}$. W. Brault of the Kitt Peak National Observatory.

The FT spectrometer of the McMath telescope at the Kitt Peak National Observatory was used in the present work. Its instrumental, single pass, unapodized resolution is $0.005 \mathrm{~cm}^{-1}$. The detector was an FW-130 photomuliplier. A brief description of the instrument is given in (3). The Raman scattering apparatus consisted of a cell designed for a slit spectrograph and used in the arrangement described in (4). The cell was placed both inside and outside the laser cavity, both laser polarizations were used, and the exciting radiation was the (multimode) argon $4880 \mathrm{~A}$ line. Pure rotational and rotation-vibrational spectra of $\mathrm{H}_{2}, \mathrm{D}_{2}, \mathrm{~N}_{2}, \mathrm{O}_{2}$, $\mathrm{CO}, \mathrm{CO}_{2}, \mathrm{C}_{2} \mathrm{H}_{2}$, and $\mathrm{C}_{2} \mathrm{~N}_{2}$ were obtained. An example is shown in Figures 2.8 which shows the spectrum of $D_{2}$ with a resolution of $0.10 \mathrm{~cm}^{-1}$ obtained in 3 nours at a gas pressure of 600 Torr. The signal to noise ratio in the pure rotation spectrum (top) is $150: 1$.

The present pilot study shows that, contrary to earlier expectations (5), Raman spectra of gases can be obtained with good signal to noise using Fourier transform spectrometers. Work is under way to improve the technique.

(1) A. Weber, "High Resolution Raman Studies of Gases" in The Raman Effect, A. Anderson, Editor (M. Dekker, Inc., New York, 1973), Vol. II, Chapter 9, pp. 543-757.

(2) Raman Spectroscopy of Gases and Liquids, Topics in Current Physics, Vol. II, A. Weber, Editor (Springer Verlag, Berlin-Heidelberg-New York, 1979). 

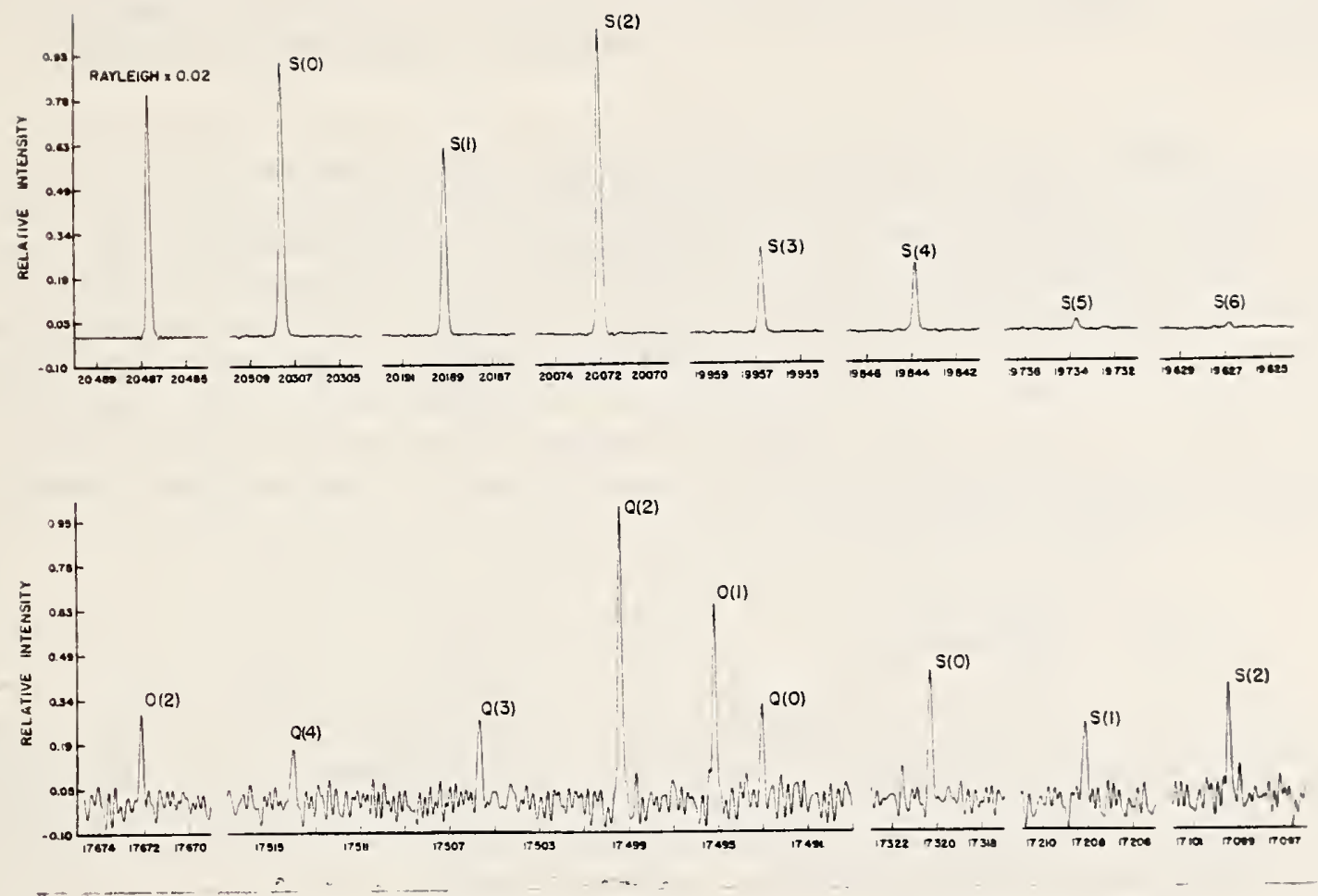

Fig. 2.8. Raman spectrum of $\mathrm{D}_{2}$ at 600 torr (Stokes side). Pure rotational spectrum (top) and fundamental band (bottom); The frequency scale is in units of $\mathrm{cm}^{-1} ; v_{0}=20486.648 \mathrm{~cm}^{-1}$.

(3) J.W. Brault, "A High Precision Fourier Spectrometer for the Visible", in Proc. ESO/CERN Conference on Auxiliary Instrumentation for Large Telescopes, S. Laustsen and A. Reiz, Editors (Geneva, 1972).

(4) A. Weber and J. Schlupf, J. Opt. Soc. Am. 62, 428 (1972).

(5) E. R. Schildkraut and T. B. Hirschfeld, "Eourier Transform Raman Spectroscopy", in Laser Raman Gas Diagnostics, M. Lapp and C. M. Penney, Editors (PIenum Press, New York, 1974) pp. 379-388.

F. Development and Applications of Theories of Molecular Spectra.

1. New Computer Programs for the Study of Asymmetric Rotor Spectra (A. G. Maki)

As a follow-up to the changes we made last year on the program that fits asymmetric rotor spectra, we have had to modisy our program that calculates synthetic spectra in order to make it compatiole with the various theoretical equations used in the fitting program. To make these modifications many of the algorithms developed for the fitting program were introduced into the structure of the spectral synthesis program. other modifications also were nade in order to make the program zore 
compatible with other computer systems. This program is essential to our studies of atmospheric molecules as it is used to calculate spectra from preliminary constants in order to extend the assignments of spectral details.

One task of our studies of atmospheric molecules has been to understand the spectrum of nitric acid between $850 \mathrm{~cm}^{-1}$ and $920 \mathrm{~cm}^{-1}$. This region includes absorption features due to two bands, $v_{5}$ and $2 v_{g}$, which are coupled through a resonance. In order to more completely understand the spectrum we have written a computer program which can be used to fit these two interacting bands. In the coming year this program will be used to help in the analysis of the spectrum of nitric acid and will also be used to help produce an atlas of observed and calculated transition frequencies and intensities for use by laboratories studying atmospheric field measurements.

2. Spin-Orbit Interaction in $\mathrm{XY}_{4}$ Molecules ( $J$. T. Hougen)

The rotational energy levels of electronic states of open shell $X_{4}$ molecules can be expected to exhibit effects due to: (i) tetrahedral splittings, (ii) spin-orbit interaction, and (iii) the Jahn-Teller effect. The effects of spin orbit interaction form the subject of the present work, which is summarized below.

Rotational energy levels in vibronic ground states of ${ }^{2} \mathrm{~A}$, ${ }^{2} E$ and. ${ }^{2} F$ electronic states of open shell $X Y_{4}$ molecules, as well as rotational line intensities for allowed transitions between such states, are discussed, including the effects of spin-orbit interaction and tetrahedral splittings. Jahn-Teller effects are assumed to be small, and are only taken into account implicitly, through their contributions to various parameters in the ef fective Hamiltonian. Qualitative information is obtained by considering several limiting-case coupling schemes among the electron spin angular momentum $S$, the electron orbital angular momentum $\underset{\sim}{L}$, and the pure rotational angular momentum $\underset{R}{\mathrm{R}}$. These limiting cases are similar in spirit to iund's coupling cases in diatomic molecules, but differ sufficiently from the latter to make detailed correspondences unhelpful. Quantitative information on rotational energy levels and line intensities is obtained numerically by diagonalizing a Hamiltonian matrix set up in a basis set characterized by uncoupled molecule-fixed projections of $S, \underset{\sim}{\mathrm{L}}$ and the total angular momentum $\mathrm{J}$, and symmetrized so that all basis set functions belong to a definite species in the subgroup $D_{2 d}$ of the true point group $T_{d}$. Hamiltonian matrix elements are determined by ladder operator techniques. Three sample spectra, corresponding to $P\left({ }^{2} F_{2}\right)-s\left({ }^{2} A_{1}\right), d\left({ }^{2} E\right)-p\left({ }^{2} E_{2}\right)$ and $d\left({ }^{2} F_{2}\right)-p\left({ }^{2} F_{2}\right)$ were caiculated. As one might expect, when the spin-orbit constant $A$ is set equal to zero, then both qualitative and quantitative aspects of the rotational-electronic problem in open-shell $\mathrm{XY}_{4}$ molecules can be mapped easily onto discussions of the rotation-vibration probiem from the $\mathrm{CH}_{4}$ literature. 
3. Group Theoretical Treatment of Internal Rotation Tunneling in the HFDimer

(J. T. Hougen and N. Ohashi)

The first phase of the work on the (HF) 2 molecule, which consists primarily of group theoretical considerations, has now been completed. It was finished in collaboration with Dr. N. Ohashi, a guest worker from Kanazawa University in Japan, and the principal results are summarized below. Further theoretical work on the spectra of $(H X)_{2}$ species is envisaged, but the precise choice of research problem and method of attack has not yet been decided upon.

The $(\mathrm{HF})_{2}$ dimer exhibits an internal rotation tunneling process between two planar but nonlinear equilibrium configurations, during which tunneling the roles of the hydrogen-bonded and the free hydrogen atom are interchanged. This process can be represented schematically with labeled atoms as $\mathrm{H}_{1} \mathrm{~F}_{2} \mathrm{H}_{2} \mathrm{~F}_{\mathrm{b}}+\mathrm{F}_{\mathrm{a}} \mathrm{H}_{1} \mathrm{~F}_{\mathrm{b}} \mathrm{H}_{2}$, and gives rise to a permutation-inversion group $G_{4}$ containing four operations. In the present work the vibration-rotation-tunneling problem in (HF) 2 is treated group theoretically in three ways: (i) by allowing tunneling only through a trans planar $C_{2 h}$ intermediate, (ii) by allowing tunneling only through a cis planar $\mathrm{C}_{2 \mathrm{v}}$ intermediate, and ( $i$ ii) by considering the trans and cis tunneling processes both to occur, though not necessarily with the same probability. The molecular symmetry groups used for these treatments are: (i) the point group $C_{2 h}$, (ii) the point group $C_{2 v}$, and (iii) a double group, which might be thought of as $\mathrm{C}_{4}{ }^{\dagger}=\mathrm{C}_{2 \mathrm{~h}^{\dagger}}{ }=\mathrm{C}_{2 \mathrm{v}}{ }^{\dagger}$. Nonplanar tunneling paths are not considered, since the internal axis method (IAM) coordinate system used here cannot easily be adapted to nonplanar internal rotation motions in this molecule. Various details of energy level diagrams, symmetry species for operators, selection rules for spectroscopic transitions, and statistical weights are presented for the (HF) 2 tunneling problem, as well as some speculation on the general question of when point groups, permutation-inversion groups, or double groups are preferable for treating large-amplitude vibrational motion problems.

4. Summary of Group Theoretical Results for Microwave and Infrared Studies of $\mathrm{H}_{2} \mathrm{O}_{2}$.

( J. T. Hougen)

Because of renewed interest in the microwave anc infrared spectra of the atmospherically important, chemically reactive species $\mathrm{H}_{2} \mathrm{O}_{2}$, and because of the study of the $v_{2}+v_{6}$ band of this molecuie by i . 3 . oIson of our laboratories, it seemed useful to collect some results from the theoretical literature on related molecules which are applicable with only minor changes to $\mathrm{H}_{2} \mathrm{O}_{2}$. These results are summarized beiow.

Group theoretical treatments found in the literature for other molecules, based on double groups of appropriate permutation-inversion groups, were modified slightly and applied to $\mathrm{H}_{2} \mathrm{O}_{2}$. This modification permits a more unified view of many theoretical results derived in rarious earlier studies of the molecule. Briefly, if no effects due to internal 
rotation tunneling were observed in the $\mathrm{H}_{2} \mathrm{O}_{2}$ spectrum, the molecule could be treated using the $\mathrm{C}_{2}$ point group of its equilibrium geometry. If effects due to internal rotation tunneling through only the trans barrier are observed (as is presently the case), the molecule should be treated using the $\mathrm{C}_{2 \mathrm{~h}}$ point group of its trans planar conformation at the top of the tunneling barrier. If effects due to internal rotation through both the trans and cis barrier can be detected, it will be necessary to treat the molecule using a double group $\mathrm{C}_{2 h} t$ of the $\mathrm{C}_{2 h}$ point group. Vibrational, torsional and rotational basis function, symmetry species, energy level diagrams, selection rules for electric dipole transitions and perturbations, etc. are derived here for the $\mathrm{C}_{2 \mathrm{~h}}{ }^{\dagger}$ double group, but these results can easily be converted to those for the $\mathrm{C}_{2 \mathrm{~h}}$ point group by dropping all $s$ and d subscripts. An empirically discovered successful fitting procedure for the ground state rotational levels, reported in a treatment of microwave and millimeter wave measurements on $\mathrm{H}_{2} \mathrm{O}_{2}$, can be rationalized on the basis of the present theoretical results.

\section{G. Microwave Spectra}

The general aim of this effort is to develop experimental and theoretical methods which can be used by other laboratories, and to make use of our unique capabilities to provide specific data cn substances of unusual interest. The current emphasis is on a) providing laboratory rest frequencies for radio telescope investigations of molecules in stellar and interstellar environments, b) senerating and investigating the spectra of weakly bound molecules to determine their structure and molecular properties, and c) developing techniques which employ microwave spectroscopy as a diagnostic tool to confirm the synthesis (existence) and structural properties of unusual (and in some cases unstable) molecular species.

\section{Millimeterwave Study of Peroxynitric Acid ( $\mathrm{HOONO}_{2}$ )}

(R. D. Suenram and F. J. Lovas)

The peroxynitric acid molecule is thought to be a temporary sirk for odd nitrogen species in the complex stratospheric chemistry. Until now no high resolution rotational or vibration-rotation spectrum has been obtained on $\mathrm{HOONO}_{2}$, thus preventing any atmospheric measurement to verify its presence and concentration. Earlier this year we began a microwave study of this species in order to characterize the rotational spectrum and determine the molecular structure.

The spectrometer employed was a Stark mocuiated millimeterwave spectrometer with a parallel plate absorption cell. Small samples of $\mathrm{HOONO}_{2}$ were produced by adding $-0.2 \mathrm{gm} 3 \mathrm{~F}_{4} \mathrm{NO}_{2}$ to about $1 \mathrm{ml}$ of 90 percent $\mathrm{H}_{2} \mathrm{O}_{2}$. This mixture was cooled to $0^{\circ} \mathrm{C}$ and was admitted directly into the absorption cell. The vafor produced in the reaction was then pumped slowly through the cell.

We expected $\mathrm{HOONO}_{2}$ to exhibit a typical a- and b-type asymetric rotor spectrum. However, we found it to be more complicated as illustrated in Fig. 2.9. For each a- or b-type transition we observed three lines, a closely spaced doutlet and a single line displaced to higher 
Prequency. We have assigned the a- and b-type doublet structure to tunneling levels of the gauche conformation of $\mathrm{HOONO}_{2}$. In addition c-type transitions which occur between rotational levels of the two tunneling states were assigned. These allowed the determination of the differences in energy of the two states. The energy difference obtained is 1779.8 $\mathrm{MHz}$. The singlet spectrum has been assigned to a low energy vibrationally excited state, most Iikely the $\mathrm{N}-0-0$ in-plane bend, which lies about 100 $\mathrm{cm}^{-1}$ higher in energy than the ground state. The tunneling splitting for the excited state transitions is too small to be resolved, however we have assigned c-type transitions for the vibration state and have determined an energy difference of $475.6 \mathrm{MHz}$ for the tunneling states.

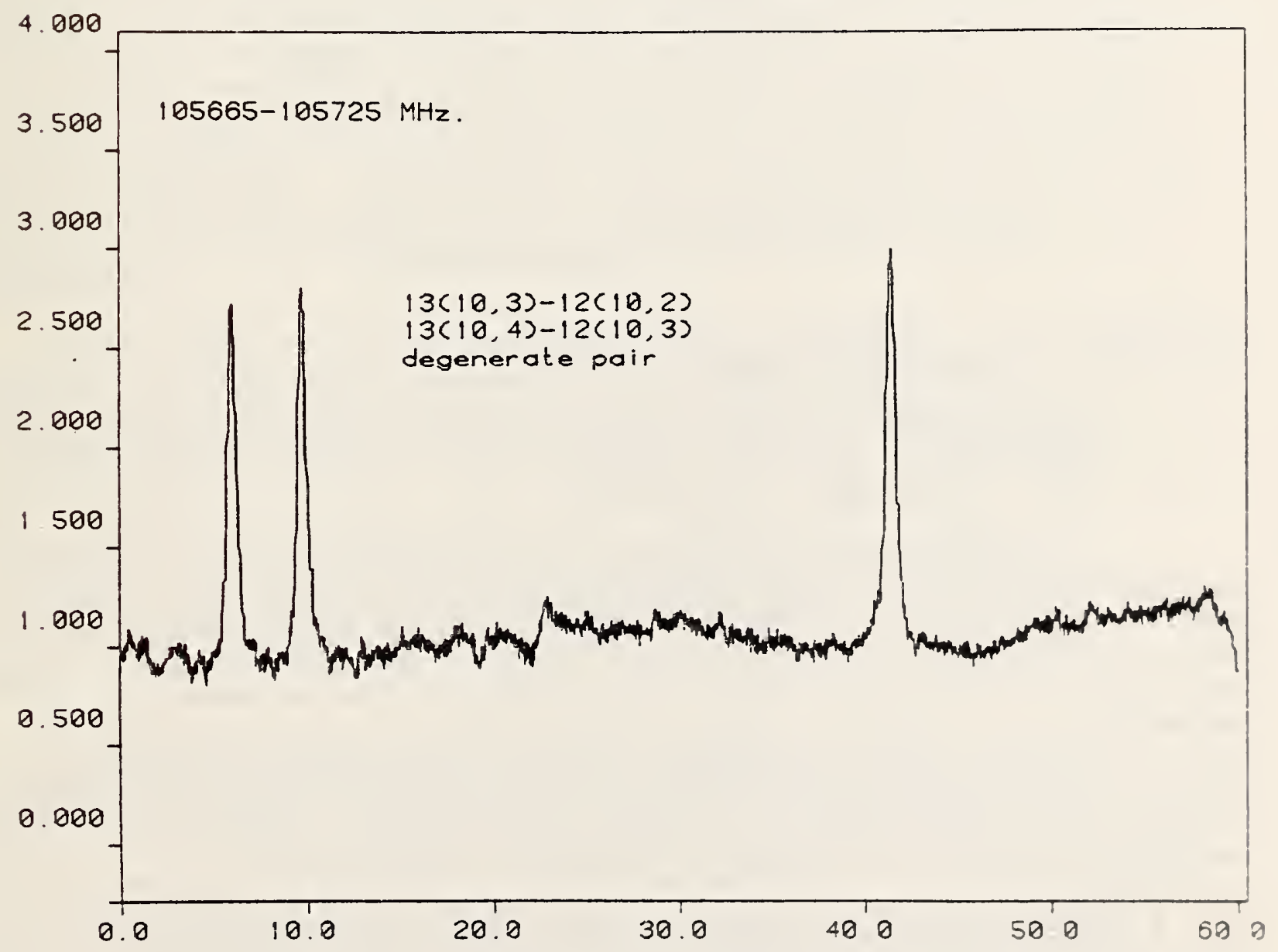

Fis. 2.9. Spectral scan of the $J=13-12, K=10$ a-type transition of peroxynitric acid. The lower frequency doublet is assigred to the ground state and the singlet to a low energy vibration. 
The qualitative molecular structure is illustrated in Fig. 2.10. All the $O$ and $N$ atoms $I$ ie in the $a, b-p l a n e$ and the proton tunnels between $a$ position above or below the $a, b$-plane, giving rise to the doubling of the rotational transitions. Studies of enriched isotopic substituted forms will be needed to obtain a precise structure.

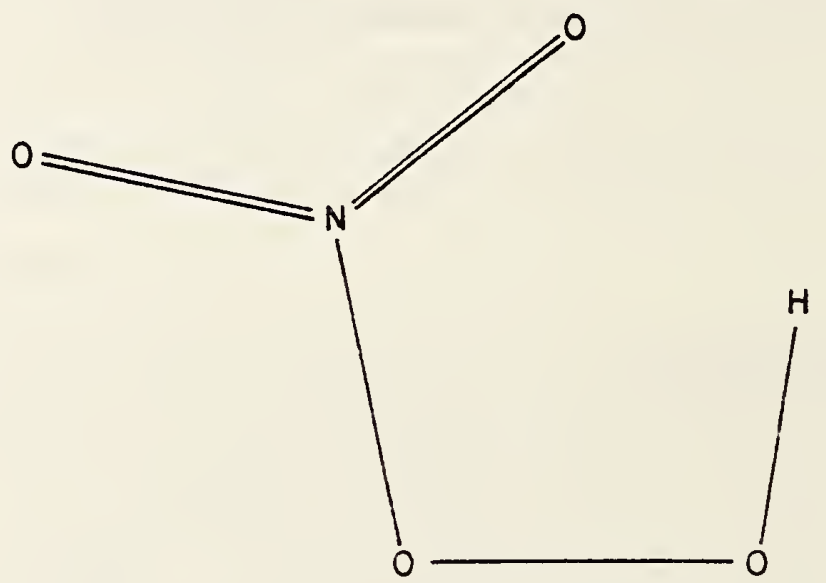

Fig. 2.10. Approximate structure of $\mathrm{HCONO}_{2}$.

2. Millimeterwave Study of Hyorogen Bonded $\mathrm{H}_{2} \mathrm{CO}-\mathrm{HF}$; The Deuterated Isotopes

(E. J. Lovas and R. D. Suenram)

Recently Baiocchi and Klemperer (1) have reported the microwave spectrum of $\mathrm{H}_{2} \mathrm{CO}-\mathrm{HF}$ from a molecular beam electric resonance study. Last year we have examined this system with a conventional waveguide starkmodulated absorption cell under thermal equilibrium conditions and obtained further information about higher energy states which are not accessible in a cooled beam experiment. With the aid of the molecular beam data we have identified numerous a-type R-branch transitions up to $\mathrm{J}=13$ and $\mathrm{K}=10$. A combined analysis of the molecular beam spectrum and the present data requires inclusion of several $\mathrm{P}^{6}, \mathrm{P}^{0}$ and $\mathrm{P}^{10}$ terms in the watson Hamiltonian in order to fit the higher $K$ transitions.

A similar study of the isotopic forms $\mathrm{H}_{2} \mathrm{CO} \cdot \mathrm{DF}$ and $\mathrm{D}_{2} \mathrm{CO} \cdot \mathrm{HF}$, which were not studied in the beam experiment, has been carried out. After correcting the rotational constants for the large changes due to the $0 \cdots \mathrm{HF}$ stretching and bending vibrations, we have derived the structure parameters given below: The geometry of the monomer units ( $\mathrm{H}_{2} \mathrm{CO}$ and $\mathrm{HF}$ ) were fixed at the values reported in the literature and only the $0 \cdots H$ distance and $\mathrm{CO} \cdot \mathrm{HF}$ angle were fitted to the corrected moments of inertia. The values obtained are: 


$$
\begin{aligned}
& r(O \cdot \cdot H)=1.780 \pm .035 \AA \\
& <C O \cdot \cdot F=104.2^{0}+2.5^{0}
\end{aligned}
$$

These may be compared to the values reported by Baiocchi and Klemperer derived from only ground state $\mathrm{H}_{2} \mathrm{CO} \cdot \mathrm{HF}: \quad r(\mathrm{O} \cdot \mathrm{H})=2.794 \mathrm{~A}$ $\angle C O \cdot F=109.5^{\circ}$

(1) F. A. Baiocchi and W. Klemperer, J. Chem. Phys. 78, 3509 (1983).

3. Pulsed Beam Fabry-Perot Cavity Fourier Transform Microwave Spectrometry.

(F. J. Lovas and R. D. Suenram)

During the past year much of our efforts have been directed at completing the construction of a Fourier Transform Microwave Spectrometer similiar to the original design by Balle and Flygare (1). Our spectrometer consists of $36 \mathrm{~cm}$ diameter spherical mirrors for the Fabry-Perot cavity which provide a loaded $Q=1-3 \times 10^{4}$ over the range of $4-18 \mathrm{GHz}$. The cavity is placed in a cylindrical vacuum chamber $56 \mathrm{~cm}$ in diameter and $1 \mathrm{~m} 10 \mathrm{ng}$. High speed pumping is attained with an $8000 \mathrm{l} / \mathrm{s}$ diffusion pump packed by a $90 \mathrm{cfm}$ roughing pump. The microwave pulse is coupled into and out of the cavity with co-axial loop antennae. All microwave components are of the co-axial type, thus eliminating the need for waveguide to co-axial transitions.

Initial tests of the spectrometer are now in progress. A time domain trace of the $J=1 \rightarrow 0$ emission signal of the 4 percent OCS in Ar carrier through a $50 \mu \mathrm{m}$ diameter nozzle (continuous beam) is shown in Fig. 2.11. The spectrum was produced from 100 microwave pulses of -0.5 us duration and digitized at 0.5 us per point for each pulse. The Fourier transformed power spectrum of the data in Fig. 2.11 is shown in Fig. 2.12. It is typical of this type of spectrometer that each transition appears as a doublet due to Doppler effects of an unskimmed beam. The true transition frequency occurs at the minimum between the two peaks. Due to slow delivery from the vendor we have not yet used the pulsed nozzle beam source.

The major direction of the studies to be carried out with this instrument will be measurement of weakly bound species, with major emphasis on hydrogen bonded molecular species. We are therefore also carrying out tests of the instrument on the van der Waals molecule Ar. $\mathrm{HCl}$ with the same nozzle employed for the OCS measurement. The Ar HC2 complex was first studied by Novick et al. $(2,3)$. It has a linear equilibrium geometry dipole moment of 0.8 Debye and dissociation energy of about 180 $\mathrm{cm}^{-1}$ ( $\left.-0.5 \mathrm{kcal} / \mathrm{mole}\right)$. We have examined the hyperfine structure of the $\mathrm{J}=2-3$ transition near $10068 \mathrm{MHz}$.

The frequency agreement of both the OCS and Ar HCl measurements with previous molecular beam electric resonance measurements is $2 \mathrm{kHz}$.

(1) T. J. Balle and W. H. Flygare, Rev. Sci. Instrum. 52, 33 (1981). 
PAUSE -- 〈Cr to continue

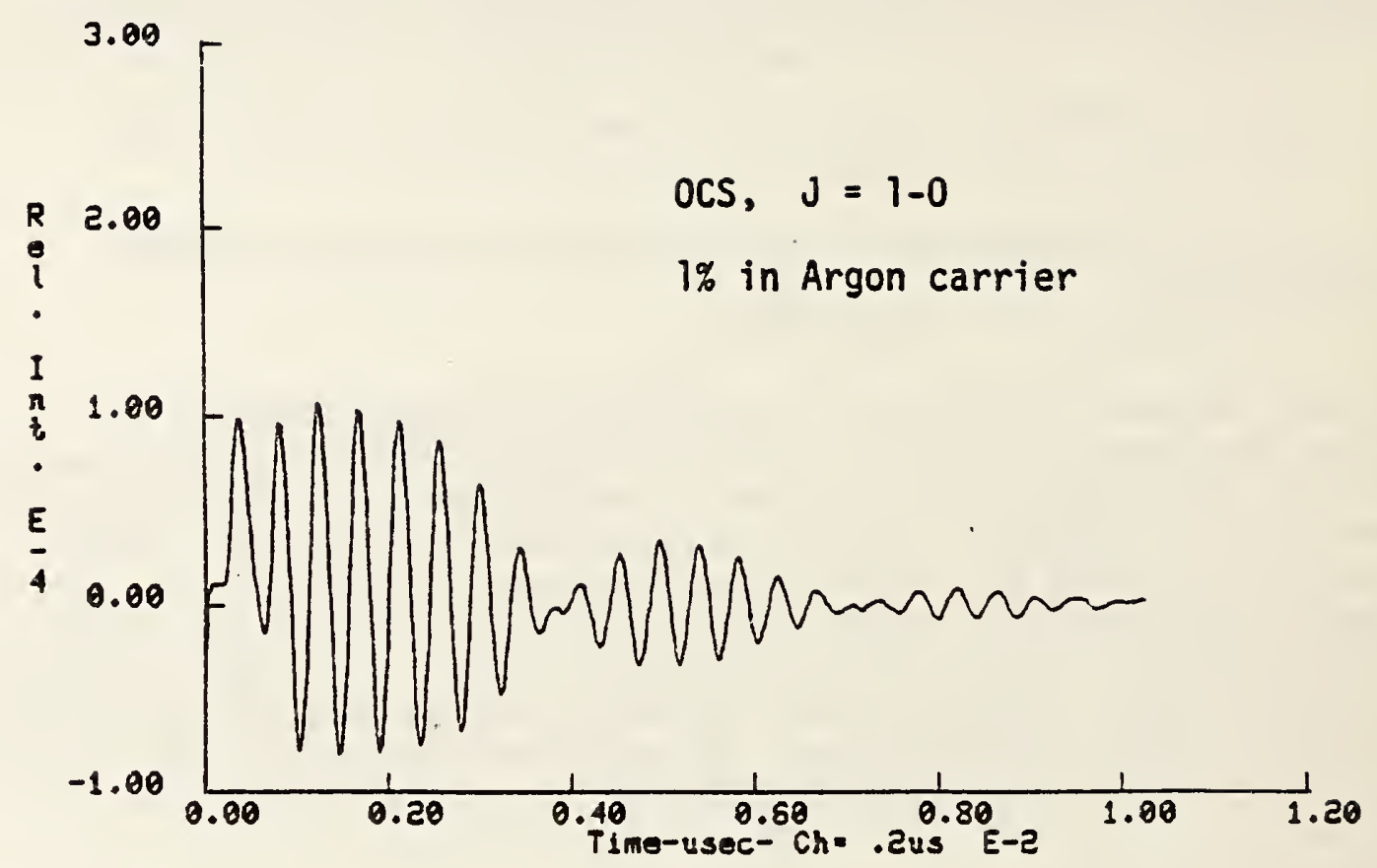

Fig, 2.11. Time domain spectrum of the $J=10$ transition of 1 percent ocS in Argon carrier gas.

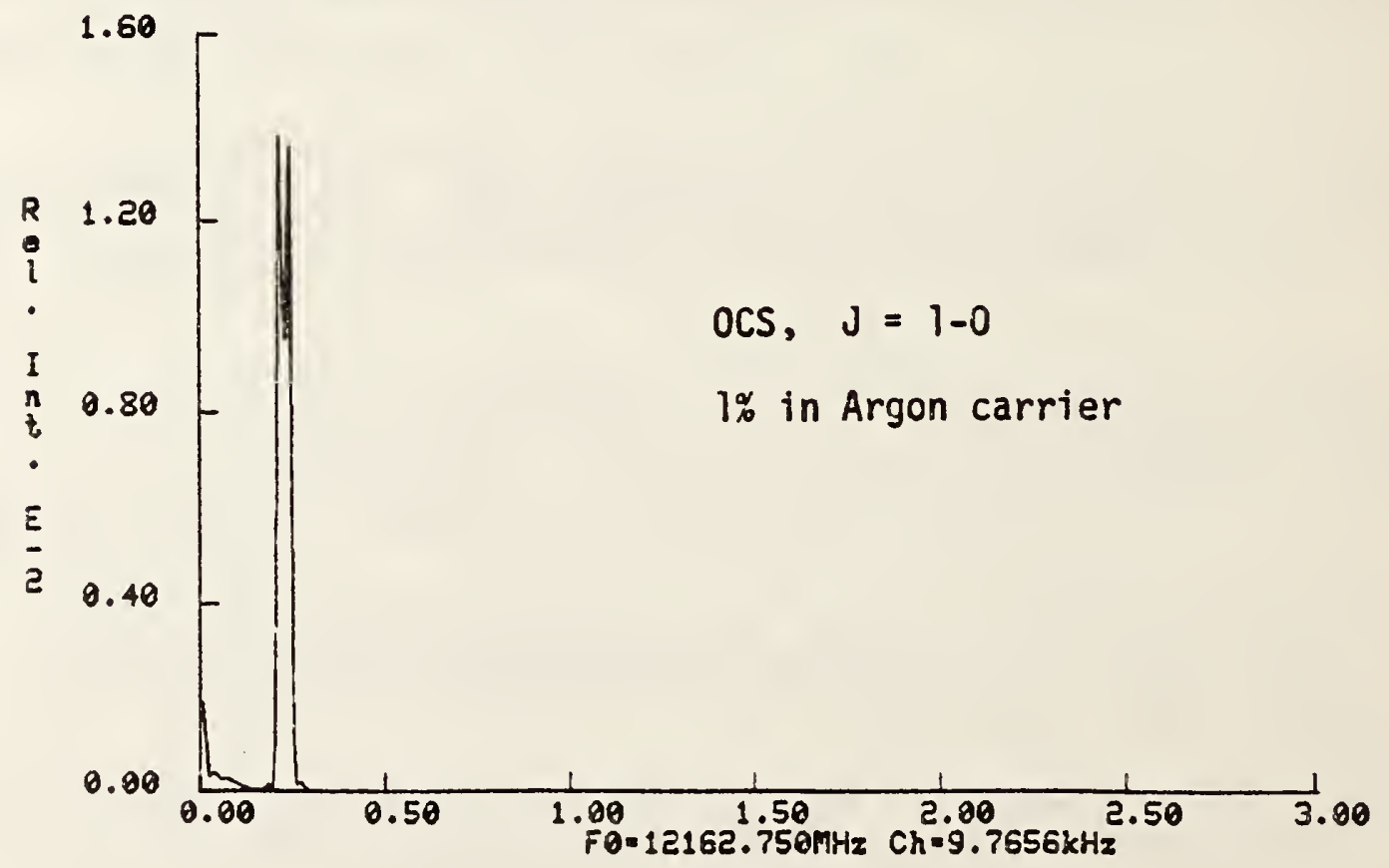

Fig. 2.12. Fourier transform of data in $\mathbf{F i g} .2 .12$, the $J=1-0$ line of ocS. 
(2) S. E. Novick, P. Davies, S. J. Harris and W. Klemperer, J. Chem. Phys. 59, 2273 (1973).

(3) S. E. Novick, K. C. Janda, S. L. Holmgren, M. Waldman and W. Klemperer, J. Chem. Phys. 65, 1114 (1976).

\section{Laboratory Microwave Studies}

Several manuscripts describing recently completed experiments on $\mathrm{HOONO}_{2}, \mathrm{H}_{2} \mathrm{CO} \cdot \mathrm{HF}$ and $\mathrm{CH}_{2} \mathrm{FOH}$ will be finished in $\mathrm{FY} 85$. Further microwave studies of isotopic forms of $\mathrm{HOONO}_{2}$ will be started and measurements of its vibrational spectrum in the far-IR region will be attempted.

Most of our laboratory effort will be directed at studies of new hydrogen bonded complexes with the Fourier transform spectrometer after completing tests with previously analyzed complexes.

H. Molecular Spectra Data Center

(F. J. Lovas, A. G. Maki, W. J. Lafferty, W. B. Olson, G. Rotter, R.

D. Suenram)

The objective of the Molecular Spectra Data Center is to collect, critically evaluate, and disseminate molecular spectra frequencies and constants obtained from molecular spectra in the microwave and infrared spectral regions. Publications of critically evaluated spectral data are designed to facilitate applications in kinetics, chemical analysis, atmospheric constituent measurement, radio astronomy and spectroscopic research. Publications of critically evaluated spectral data of both broad and specialized interests are produced in this project.

Since this project was initiated, two major compilations of critically evaluated microwave spectra data have been published. These are parts I and II of the Microwave Spectral Tables on diatomic and triatomic molecules, respectively. Other specialized critical reviews have been completed for the series entitled "Micrcwave Spectra of Molecules cf Astrophysical Interest." To date, 21 papers on 26 of the interstellar molecules have been published; approximately half of these investigations were carried out at NBS and the remainder completed under the guidance of the project. Gas-phase molecular structures have been critically evaluated. A list of recommended rest frequencies for observed intersteliar molecular lines has been prepared.

The development of irequency standards in the infrared spectral region involves both precise new laboratory measurements and cricical analysis of both microwave and infrared spectra. High resolution infrared spectroscopy is now employed in diverse technological areas, such as, industrial process monitoring, stack emission measurements, atmospheric constituent measurements, combustion studies and gas dynamics. 
1. Revision of Recommended Rest Frequency List.

In 1979 we published a paper in the Astrophysical Journal Supplement Series entitled, "Recommended Rest Frequencies for Observed Interstellar Molecular Transitions." This was received and used with enthusiasm by radio astronomers. During the past five years a dozen new species and numerous new transitions ( $>700$ ) of previously known species have been reported. Since this nearly tripled the previous information, an update of this information was deemed appropriate. This effort began with the selection of recommended frequencies and coding all the pertinent information on the new transitions. The data in the prior publication was checked for revisions, coded and added to this new data file during the current year. An extensive reference file of both laboratory measurements and astronomical reports has also been coded for eventual computer typesetting.

The final editing of these files is now in progress. In order to insure both complete coverage and accuracy, we have sent preliminary copies of the list to all the major radio astronomy groups and facilities to solicit unpublished observations to be incorporated.

\section{IR Calibration Atlas 500 to $720 \mathrm{~cm}^{-1}$.}

This project is part of our effort to develop infrared frequency standards compilations for use in calibrating tunable IR laser spectrometers. Scme very accurate data on OCS, $\mathrm{N}_{2} \mathrm{O}$, and $\mathrm{CO}_{2}$ have been obtained from literature publications and via new measurements in the longer wavelength region. Art Maki spent several weeks at the University of Culu in Finland with Dr. Kauppinen to obtain some of the new measurements on these species with the long wavelength Fourier transform spectrometer at Oulu. The analysis of both these IR data and accurate microwave measurements is underway and progressing well.

\section{Microwave Spectral Tables-Hydrocarbons}

The publication of a third part of the microwave spectral tables is planned. The fitting of the species which can be classified as normal asymmetric rotors is progressing well and should be completed by the end of the current fiscal year. We have also writien a pitting program to analyze symmetric top species of which there are several in this group. About 15-20 hydrocarbon species exhibit internal rotation splittings and thus, are more difficuit to analyze. We are currently implementing fitting codes to treat asymmetric rotors with internil rotation. Approximately 70 hydrocarbon species will be included in the review.

\section{Future Plans}

As the foregoing activity report of the High Resolution Spectroscopy Group indicates it is engaged in providing frequency standards and critically evaluated reference spectroscopic data, and in transferring forefront spectroscopic expertise to the user community. It also is 
engaged in advancing spectroscopic measurement methods and techniques, in developing models, theoretical concepts and methods for predicting molecular properties and interactions and in conducting research to understand and model fundamental chemical processes. We will continue in these endeavors during the coming years.

Specifically, the substance of the group's activities is in the areas of development of infrared frequency standards for the calibration of laser spectrometers, the critical evaluation of spectroscopic data, spectroscopy of atmospheric trace gases, spectroscopy of weakly bound molecular complexes, and development of experimental techniques and instrumentation.

The development of frequency standards suitable for the calibration of infrared laser spectrometers and the critical evaluation of spectral data will continue to play a significant role. The needs for infrared frequency standards are well known and the philosophy of our frequency standards development program has been repeatedly stated. Briefly, this is to furnish to the user community accurate absorption standards based on readily available and easy to handle molecular gases so as to obviate on the part of the user any specialized instrumentation or procedures for the calibration of his spectrometer. Such standards guarantee their most widespread use. A very detailed assessment of the present state of affairs and plans for the immediate future has been given in section $B .3$ of this report.

The Molecular Spectra Data Center is part of the National Standards Reference Data System and operates under the aegis of the Bureau's separate Office of Standard Reference Data. The activities of the Data Center are focussed on microwave spectral data and infrared frequency standards compilations.

A manuscript on the revised "Recommended Rest Frequencies for Observed Interstellar Molecular Transitions in the Microwave Region" will be completed near the beginning of FY 85. Efforts to incorporate new observations will continue up to production of the publication in order to keep the information content as current as possible.

The final analyses and manuscript of the MMicrowave Spectral Tables. part III Hydrocarbons" will be completed in early FY 85. In parallel with this publication a magnetic tape of the measured and calculated spectra of the most abundant isotopic form will be produced for distributicn to interested users on request. This is part of the long range plan to develop a microwave spectrai line atlas, so care is being iaken to keep all the coding compatible with the previous files developed. It should be noted that we continue to maintain and update the computer files on the groups of species previously published, i.e., diatomics and triatcmics.

The infrared frequency standards compilation will benefit from new microwave measurements that have just become available. These will be used to improve some of the tables. We plan to include in the compliation figures showing the spectrum in each frequency interval covered by the 
tables. Many workers feel that such figures will make it much easier to use the tables. Because of difficulties encountered in obtaining real spectra suitable for this purpose, the atlas will probably include figures that are generated from the tables by a computer plotter. Since the tables will contain all of the strong lines and most of the weak lines, such a synthetic spectrum will closely match a real spectrum. of course, we have real spectra which will be used to verify the accuracy of the synthetic spectrum. One advantage of plotting a synthetic spectrum is the flexibility in specifying several effective spectral intensities (or pressure pathlength products) in order to illustrate the wide range of intensities that we shall have in the tables. Completion of the manuscript "IR Calibration Atlas from 500-720 $\mathrm{cm}^{-1}$ " will occur in FY 85 .

Our activities in the area of trace atmospheric species are in support of other agency programs, notably those of NASA and CMA. The thrust of this work is to provide spectroscopic data for those trace atmospheric species that play a significant role in controlling and modifying the properties of the earth's atmosphere. The needs of these programs are of long duration and we expect not only to continue to provide accurate data and expertise to them but also hope to somewhat expand our efforts through additional other agency contracts.

Two years ago the group initiated a new phase of research in the high resolution spectroscopy of weakly bound molecular complexes. Our initial study on the hydrogen bonded $\mathrm{HF}$-dimer molecule was the first such work ever reported in which a rotation-vibration band of a hydrogen bonded complex was fully resolved. This work has been followed by the resolution of the HC2-dimer spectrum and of the spectrum of the Argon-HCl van der Waals complex as reported in sections $D .2$ and 3 . The availability of the high resolution Fourier transform spectrometer and the pulsed molecular beam Fabry-Perot microwave spectrometer permits us to expand our activities in the study of weakly bound complexes to the far infrared and microwave region of the spectrum. Based on this total spectroscopic capability, which spans the region fran $4000 \mathrm{~cm}^{-1}$ to $10 \mathrm{~cm}^{-1}$, we will continue in the coming years to work on the spectra of hydrogen-bonded and van der Waals molecular complexes. As part of these efforts we will continue to improve and advance our instrumental capabilities and techniques. 
M. P. Casassa, R. R. Cavanagh, L. B. Elwell, E. J. Heilweil, M. E. Jacox, D. S. King, D. A. Mantell, J. Perry, J. C. Stephenson, and A. Woodward

\section{A. Introduction}

This group primarily does quantum-state specific studies of molecular dynamics. A common factor in the research described below is the role of energy transfer in the spectroscopy and kinetics of molecules.

We have been fortunate in having ive post-doctoral iellows (Casassa, Heilweil, Mantell, Perry, Woodward) join the group in the last few months. Because of the difficulty of the picosecond studies of energy transfer, and the ultra high vacuum thermal desorption experiments, the new postdoctoral scientists are particularly important to the success of this research.

\section{B. Picosecond Studies of Molecular Energy Transfer}

(M. P. Casassa, R. R. Cavanagh, L. B. Elwell, E. J. Heilweil,

J. Perry, J. C. Stephenson, A. Woodward)

The major effort in the picosecond chemistry program has been directed at the study of vibrational energy transfer (VET). During the past year we have successfully measured the rates of energy transfer from vibrationally excited chemical bonds in liquids, solids, and in molecules bound to surfaces.

We used an infrared pump/probe method to do time-resolved measurements of the vibrational energy relaxation race $\left(T_{1}{ }^{-1}\right)$ for hydroxyl groups chemisorbed on a silica $\left(\mathrm{SiC}_{2}\right)$ surface in a variety of chemical environments. Previously, energy transfer rates have been measured for the vibrations of many molecules in the gas phase, and for a $f \in w$ molecules in liquids and low temperature solids. However, there have been no previous vibrational energy transfer measurements for vibrations on surfaces.

Our initial measurements were on the surface hydroxyls (OH) bound to dispersions of colloidal fumed silica (Degussa Aerosil 200, 120 A diameter particles, $200 \mathrm{~m}^{2} / \mathrm{gm}$ surface area) suspended in Iiquid, $\mathrm{CC}_{4}$ at room temperature. Inirared absorption in the $3000-4000 \mathrm{~cm}^{-1}$ region is due to isolated surface hydroxyl groups $\left(\mathrm{v}=0 \rightarrow \mathrm{v}=1\right.$ at $\left.3691 \mathrm{~cm}^{-1}\right)$, and physisorbed molecular water $\left(=3400 \mathrm{~cm}^{-1}\right)$.

The picosecond apparatus used in this investigation was based on a modelocked $\mathrm{Nd}^{+3}$ : YAG laser. Single $1.06 \mu \mathrm{m}$ pulses of $-35 \mathrm{~mJ}$ and $10 \mathrm{~Hz}$ repetition rate pumped a chain of three $\mathrm{LiNbO}_{3}$ crystals to parametrically zenerate tunable infrared picosecond radiation, which had a spectral bandwidth of $10 \mathrm{~cm}^{-1}$ FWHM, duration of $14 \mathrm{ps}$ FitH, and delivered about $200 \mathrm{HJ}$ of pump energy to the sample.

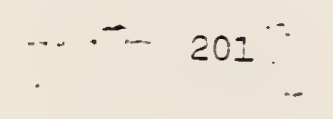


The surface hydroxyl $v=0 \rightarrow 1$ transition was excited with a strong IR pulse which transferred up to 10 percent of the hydroxyls from the $v=0$ to $\mathrm{v}=1$ level. Due to anharmonicity, $\mathrm{OH}(\mathrm{v}=1)$ does not absorb at the pump frequency, so excitation causes a transient increase in sample transmission, $T$. As population relaxes, the sample transmission returns to its equilibrium value, $T_{O}$; $\ln \left(T / T_{O}\right)$ is proportional to $\mathrm{OH}(\mathrm{V}=1)$ population. Recovery of sample transmission was monitored by a weak, time-delayed probe pulse $(-5 \mu \mathrm{J})$ derived from the saturating pulse and a variable optical delay. At each delay $\left(t_{D}\right)$, the probe pulse transmission was measured to 0.5 percent accuracy with pump excitation and with the pump blocked using a dual pyroelectric detector energy ratiometer. A typical data run is shown in Figure 3.1 .

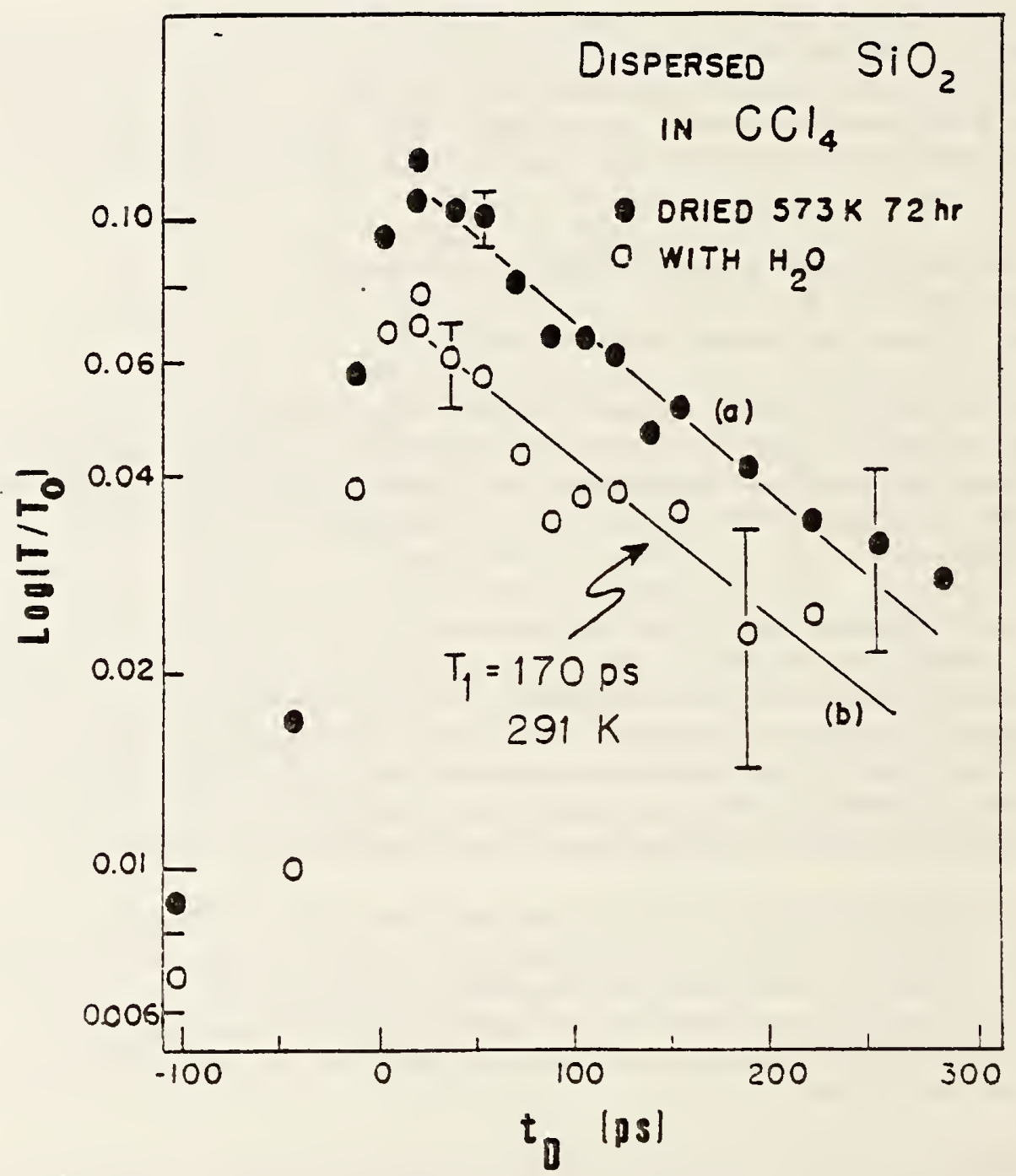

Fig. 3.1. Vibrational population relaxation $(v=1)$ of surface hydroxyl groups on silica dispersed in $\mathrm{CCl}_{4}$ at $291 \mathrm{~K}$. Typical data for (a) dried sample and (b) sample containing adsorbed water exhibit simizar lifetimes of 170 ps from least squares fits. 
The surface hydroxyl $\mathrm{v}=1$ population for silica in $\mathrm{CC}_{4}$ decayed exponentially with a characteristic time constant $\mathrm{T}_{1}=150 \pm 20 \mathrm{ps}$. For sllica suspended in the solvent $\mathrm{CF}_{2} \mathrm{Br}_{2}, \mathrm{~T}_{1}=120 \mathrm{ps}$, and for the solvent $\mathrm{CH}_{2} \mathrm{C}_{2}$ the surface hydroxyl population decayed with $\mathrm{T}_{1}=100 \mathrm{ps}$.

A pressed disc (same surface area but no solvent) of the material gave a relaxation time $T_{1}=220 \mathrm{ps}$ for the $\mathrm{OH}$ vibration $\left(3750 \mathrm{~cm}^{-1}\right)$ at the surface-vacuum interface. This same $T_{1}$ was observed for the sample at room temperature and cooled to $\mathrm{T}=-185 \mathrm{C}$.

A sample was prepared in which 70 percent of the surface OH groups were deuterated (converted to OD). $T_{1}$ for the $\mathrm{OH}(V=1)$ species in this deuterated sample suspended in $\mathrm{CC}_{4} 4$ was the same as for the ordinary non-deuterated sample. A sample with substantial coadsorbed $\mathrm{H}_{2} \mathrm{O}$ also gave this same $T_{1}$.

Although we have not yet directly determined where the $O H$ vibrational energy goes, we may draw some conclusions from the preceding observations. Most or the $3700 \mathrm{~cm}^{-1}$ of energy must be deposited in the $\mathrm{SiO}_{2}$ lattice. If this energy were to be transferred to the internal modes of the solvents, a five quantum number change $(\Delta v \geqq 5)$ would be required in $C C 2_{4}, \Delta v \geqq 4$ in $\mathrm{CF}_{2} \mathrm{Br}_{2}$, or $\Delta \mathrm{V} \geqq 2$ in $\mathrm{CH}_{2} \mathrm{Cl}_{2}$. Intermolecular VET processes requiring large $\Delta \mathrm{v}$ are expected to be improbable. However, collisions with the solvent molecules may induce energy transfer from the $\mathrm{OH}(\mathrm{V}=1)$ to the $\mathrm{SiO}_{2}$ substrate (intramolecular energy transfer). The presence (or absence, in the deuterated sample) of neighboring surface OH groups or coadsorbed $\mathrm{H}_{2} \mathrm{O}$ is apparently not important in determining $T_{1}$. The absence of a temperature dependence of $\mathrm{T}_{1}$ at the surface-vacuum interface is consistent with theories for an intramolecular vibrational relaxation $T_{1}$ process in an isolated molecule.

Pump/probe experiments on the bulk OH absorption feature of a $1 \mathrm{~mm}$ thick quartz window produced a similar degree of saturation and a 150 ps lifetime. This result is consistent with the idea that the $3700 \mathrm{~cm}^{-1}$ of $\mathrm{OH}(\mathrm{v}=1)$ energy goes to the $\mathrm{SiO}_{2}$ lattice for the surface hydroxyls.

Spectral linewidths observed in surface vibrational spectroscopy have sometimes been interpreted as $T_{1}$ uncertainty broadening. However, the width of the $\mathrm{OH}$ absorption feature must arise from site inhomogeneities or dephasing processes, since the linewidth corresponding to $T_{1}$ for surface $\mathrm{OH}$ of $-0.03 \mathrm{~cm}^{-1}$ is 2000 times smalier than observed.

The long $T_{1}$ for these surface viorations may have chemical importance. Vibrational excitation of certain bonds greatly increases the rates of specific chemical reactions, and apparently vibrational ifetimes can be comparable to or longer than the timescales for an adsorbate to reorient, hop to an adjacent site, or diffuse many Angstroms. The energy released in a reaction, rather than dissipating instantaneously into the substrate, may remain available for energizing bonds of other reactants. 
There are many experiments underway to elucidate the pathways of energy transfer in molecules on surfaces, and to identify the important physical parameters of the substrate and adsorbate which determine the rates of VET. On theoretical grounds, and by analogy to gas phase results, we expect several factors will be important, including the match between the frequencies of the substrate and adsorbate, the distance from the surface and the orientation of the excited bond, its permanent and transition dipole moment, and the presence of coadsorbed molecules. The published IR spectra of a number of high area metal oxides suggest that we may do similar experiments on substrates such as $\mathrm{TiO}_{2}, \mathrm{ZnO}, \mathrm{Al}_{2} \mathrm{O}_{3}$, etc. Likewise, many adsorbates such as $\mathrm{Si}-\mathrm{NH}_{2}\left(\nu_{\mathrm{NH}}=3525,3447 \mathrm{~cm}^{-4}\right)$ or $\mathrm{Si}-0-\mathrm{CH}_{3}\left(\mathrm{v}_{\mathrm{CH}}=3000,2958,2858 \mathrm{~cm}^{-1}\right)$ have frequencies in the $v \geqq 2800$ $\mathrm{cm}^{-1}$ range accessible to our present apparatus.

The VET rate for molecules on the surface of conducting metals is a subject of much current debate. The mechanism of relaxation via electron-hole pair formation has been predicted to be effective, and the rather large spectroscopic linewidths of viorational transitions of molecules on metals have been interpreted as $T_{1}$ broadening. We will try to measure $T_{1}$ for molecules on small metal particles in several systems where the known IR and Raman spectroscopy suggests that our approach may be successful.

In other experiments, the relaxation times of $\mathrm{HC} 2(\mathrm{v}=1)$ molecules dilute in room temperature $\mathrm{CC}_{4}$ and $\mathrm{CC}_{3} \mathrm{~F}$ solvents were determined. As in the surface experiments, the vibrations were excited by tunable IR pulses. However, the time dependent population in the HCl $(v=1)$ was monitored by spontaneous anti-Stokes Raman scattering (SPARS) using frequency doubled Id-Yag probe pulses. The IR/SPARS method is preferable in several ways to the IR/IR saturation method: for instance, it permits study of samples where the $\mathrm{V}=1 \rightarrow \mathrm{v}=2$ excited state absorptions would make the one-color $I R / I R$ experiment difficult to interpret. For $\mathrm{HCl}(\mathrm{V}=1)$ in $\mathrm{CC}_{4}, \mathrm{~T}_{1}$ was measured as $4.7 \pm .4 \mathrm{~ns}$; for $\mathrm{CCl}_{3} F, \mathrm{~T}_{1}=6.4 \pm .9 \mathrm{~ns}$.

These results may be compared to the relaxation of the surface $\mathrm{OH}$ $(\mathrm{v}=1)$ groups in $\mathrm{CC}_{4}$ solvent. The $\mathrm{HCl}(\mathrm{v}=1)$ transition in $\mathrm{CC}_{4}$ is at 2840 $\mathrm{cm}^{-1}$, while the $\mathrm{OH}(\mathrm{v}=1)$ transition in $\mathrm{CCl}_{4}$ is at $3690 \mathrm{~cm}^{-1}$. We expect the rate of VET from surface-bound $\mathrm{OH}(v=1)$ to $\mathrm{CCl}_{4}$ to be even slower than for $\mathrm{HCl}(\mathrm{v}=1)$ to $\mathrm{CC}_{4}$, since the $\mathrm{OH}$ quantum is significantly larger. ( $\Delta V \geqslant 5$ in. $\mathrm{CCl}_{4}$ is required to accept $3690 \mathrm{~cm}^{-1} ; \Delta V=4$ to accept $2840 \mathrm{~cm}^{-1}$ ).

The deactivation rate $\mathrm{T}^{-1}$ of $\mathrm{HCl}(\mathrm{V}=1)$ by $\mathrm{CCl}_{3} \mathrm{~F}$ in the liquid is just what one would obtain by the simplest possiole extrapolation of the dilute gas phase bimolecular deactivation rate constant $\mathrm{k}\left(\mathrm{cm}^{3} / \mathrm{molec} \cdot \mathrm{sec}\right)$ to the liquid phase number density $p\left(\mathrm{molec} / \mathrm{cm}^{3}\right)$ via $\mathrm{T}_{1}^{-1}$ ( 1 iquid) $=k$ (gas)*o(liquid). The apparent success of this "isolated binary collision" (IBC) model to explain liquid phase data has been observed previousiy in studies of a few other diatomic molecules (hydrogen, HX) which have high frequency vibrations. Nevertheless, the use of gas phase rate constants to oredict rates in condensed phases is difficult to justify or to understand. We hope to do several additional experiments in liquids to determine the limits of applicability of the ISC approximation. 
This research has been supported in part by the Air Force office of Sclentific Research.

C. Desorption of Molecules from Surfaces

(R. R. Cavanagh, L. B. Elwell, D. S. King and D. A. Mantell)

A dedicated experimental facility including surface sensitive analysis (e.g., mass spectrometer, argon sputtering, Auger electron analyzer), computer controlled data acquisition and sub-doppler (150 $\mathrm{MHz}$ ) laser resolution is being applied to the study of the thermal desorption of molecules chemisorbed to well characterized surfaces. Results for No desorbing from clean $\mathrm{Ru}(\mathrm{OO} 1)$ and pre-oxidized Ru, including rotational-, spin-, and lambda doublet state population distributions, molecular orientation anisotropy, and Doppler profiles have been measured and published.

Work is currently underway on No/Pt(111). Our choice of platinum was based, in part, on the following two considerations. First, No beam scattering experiments have jeen performed on $P t(111)$ and Pt foils giving an indication for $T_{\text {rot }}$ (trapping/desorbing NO) $\approx I_{\text {surface. Second, unlike }}$ the NO/Ru system, there is little or no dissociation of the surface bound No during the desorption event. In the No thermal desorption spectrum from Pt(111) there are three prominent desorption features. We have sufficient signal-to-noise to compare the energy distribution in the desorbed NO corresponding to each distinct desorption feature. The NO/Pt(111) thermal desorption spectra have been shown to exhibit dramatic effects due to changes in coverage and the presence of co-adsorbates sucin as CO. We shall follow the dynamics over a factor of 10 change in coverage and in the presence of co-adsorbed $\mathrm{CO}$ or oxygen.

Thermal desorption probes processes occurring under "equilibrium" conditions. This is to say that a heating rate of $10 \mathrm{~K} / \mathrm{sec}$ is mucr slower than energy transfer processes. We hope in the coming year to study direct desorption following very rapid laser heating, where the heating rate may exceed $10^{12} \mathrm{~K} / \mathrm{sec}$ and compete with other important energy exchange processes.

This work is supported in part by the U.S. Department of Energy.

D. Photochemistry in Molecular Beams

(L. B. Elwell, D. S. King and J. C. Stephenson)

These experiments represent, in part, an outgrowth or work supported by the U.S. Army Research Office and the U.S. Office of Naval Research to characterize the decomposition of energetic materials. The experiments resulted in the total characterization of energy disposal in the No product fragment formed by inirared multiphoton dissociation of C.3ONC 15 both gas bulb and molecular beam environments.

For methyl nitrite, $\mathrm{CH}_{3} \mathrm{CNO}$, dissociated in a pulsed molecular beam under collisionless conditions by $\mathrm{CO}_{2}$ laser pulses of well defined intensity, the only reaction was $\mathrm{CH}_{3} \mathrm{ONO} \rightarrow \mathrm{CH}_{3} \mathrm{O}\left(\mathrm{X}^{2} \bar{E}\right)+\mathrm{NO}\left(\mathrm{X}^{2} \pi\right)$, i.e. botn products being in the ground electronic state. in a typical experiment. 
the $\mathrm{CO}_{2}$ photolysis pulse was a 50ns temporal square wave of intensity $I=400 \mathrm{MW} / \mathrm{cm}^{2}$. Ultraviolet laser excited fluorescence of the gamma (A $\mathrm{X}$ ) bands of the No fragment was carried out at $0.2 \mathrm{~cm}^{-1}$ resolution to determine the vibrational, rotational, spin, and lambda doublet-state distributions of the nascent NO. The ratio of NO $(v=1)$ to NO $(v=0)$ corresponds to a vibrational temperature $T_{v}=460 \pm 50 \mathrm{~K}$. The rotational states of NO must be considered simultaneously with the two electronic spin-orbit states, ${ }^{2} \Pi_{1 / 2}\left(F_{1}\right)$ and ${ }^{2} \Pi_{3 / 2}\left(F_{2}\right)$, which are separated by $A=123$ $\mathrm{cm}^{-1}$. The low lying $(v=0)$ rotational states $\left(E_{\text {Rot }} \leqq 1000 \mathrm{~cm}^{-1}\right)$ are populated in Boltzmann-like distributions, characterized by rotational temperatures $T_{\text {Rot }}\left(F_{1}\right)=399 \pm 9 \mathrm{~K}$ and $T_{\text {Rot }}\left(F_{2}\right)=529 \pm 97 \mathrm{~K}$ (See Figure $3.2)$. The rotations of the NO $(v=1)$ level are a little colder than for the $v=0: T_{\text {Rot }}\left(F_{1}, v=1\right)=220 \mathrm{~K}$ vs. $T_{\text {Rot }}\left(F_{1}, v=0\right)=399 \mathrm{~K}$. The ratio of total population in all $F_{1}$ nuclear rotation states to population in $F_{2}$ is 2.6 , corresponding to a spin-orbit temperature of $\mathrm{T}_{\text {so }}=180 \mathrm{~K}$. These values for product energy do not depend strongly on the intensity of the

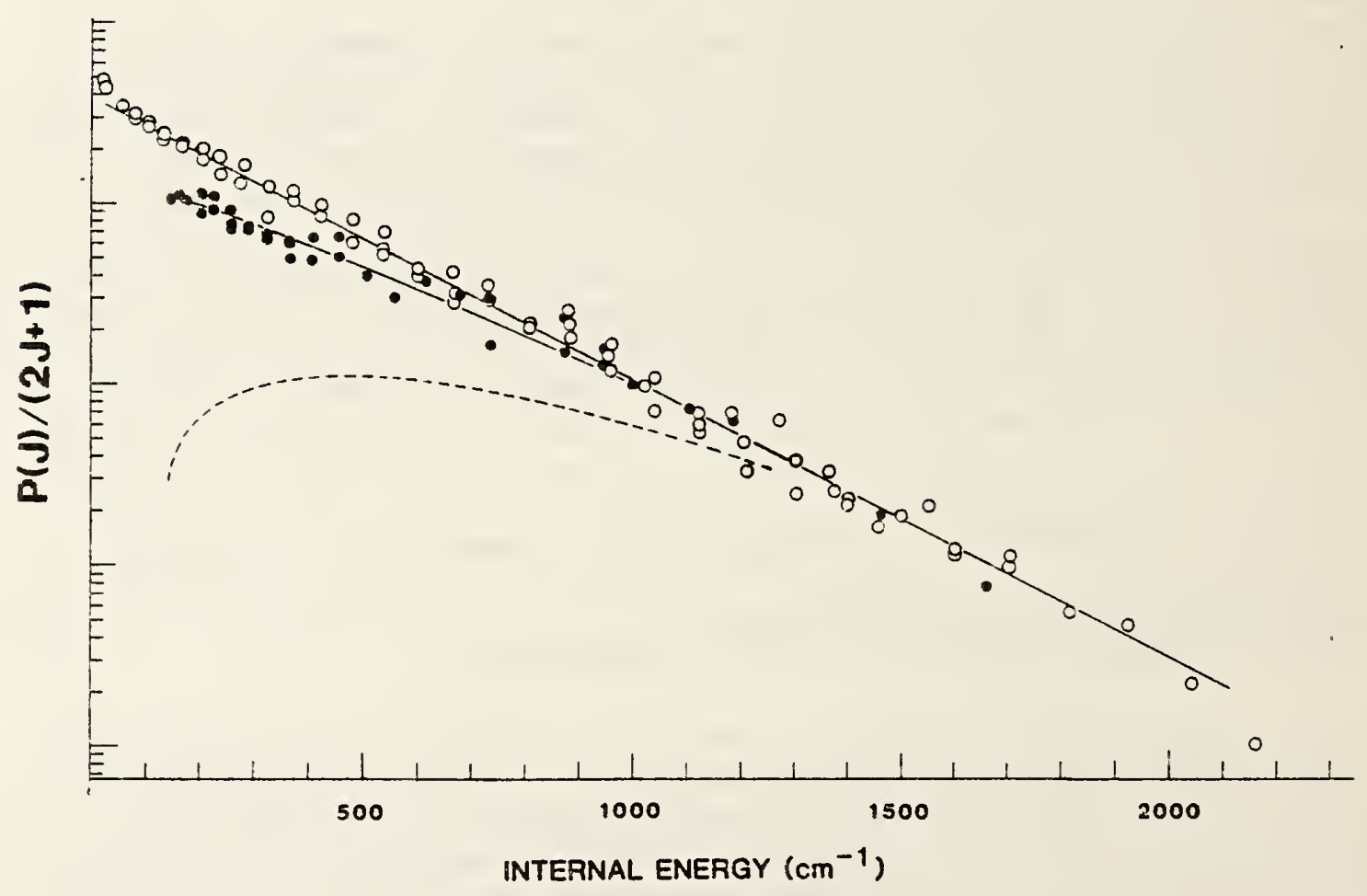

Fig. 3.2. Distribution of rotational sates in the No product formed by $\mathrm{CO}_{2}$ laser induced dissociation of $\mathrm{CH}_{3} \mathrm{ONO}$ under çollisionless conditions in a molecular beam. Open circles, $2 \Pi_{1 / 2}(\mathrm{~J})$ states; closed circles, $2 \Pi_{3 / 2}(\mathrm{~J})$ states. 
$\mathrm{CO}_{2}$ laser. Unlike the spin-orbit states, the lambda doublet states of NO are formed equally (classically, the two lambda doublets correspond to the electron density in the singly occupied pi orbital localized in, or out, of the plane of molecular rotation). Rotation of the probe laser polarization indicated an isotopic distribution of NO $\mathrm{J}$ vectors.

Experiments were also performed, in the molecular beam, at much higher resolution $\left(-0.01 \mathrm{~cm}^{-1}\right)$ to determine the Doppler widths, and hence the kinetic energy of NO initially iormed in well defined internal states. The observed spectral widths were Gaussian over two e-foldings, and when converted to center of mass translational temperatures correspond to $\mathrm{T}_{\mathrm{cm}}=$ $330 \pm 50 \mathrm{~K}$, the same for different rotational states with energy in the range $0<E_{\text {Rot }} \leqq 1300 \mathrm{~cm}^{-1}$.

These results are consistent with the usual RRKM picture of a simple bond-breaking reaction from states of internal excitation for which the laser excitation rate equals the microscopic rate of unimolecular reaction. We estimate a total of only $4 \mathrm{kcal}$ of energy (internal and kinetic) to be in the $\mathrm{CH}_{3} \mathrm{O}$ and $\mathrm{NO}$ photofragments. This is the average energy, in excess of the enthalpy required to break the $\mathrm{CH}_{3} \mathrm{O}-\mathrm{NO}$ bond, of the molecules which dissociated. A calculation consistent with the experimental Arrhenius A factor suggests an RRKM lifetime of 0.5 to 1.0 nsec for $\mathrm{CH}_{3} \mathrm{ONO}$ molecules with this excess energy. The observed statistical distribution of lambda doublets is expected for a (free rotor) transition state in which there is no distinction between cis and trans $\mathrm{CH}_{3} \mathrm{ONO}$. The observed spatially isotropic $\mathrm{J}$ distribution is expected for this process where the excitation and dissociation times greatly exceed molecular rotational times. These results contrast with many recent observations of $\mathrm{NO}$ in beam scattering and ultraviolet (prompt) photolysis experiments, where polarization and non-statisticai state distributions have been observed.

These results were for photolysis of $\mathrm{CH}_{3}$ ONO in a seeded bean where the initial internal energy of the reactant molecules was very low. The rotational temperature in the beam was $\leqq 6 \mathrm{~K}$, and the $\mathrm{CH}_{3}$ ONO was aIso significantly cooled vibrationally. However, despite this cooling, tine product energy distribution was the same for molecules photolyzed in the beam and for $\mathrm{CH}_{3}$ ONO photolyzed in a room temperature bulb, where the initial reactant rotational and vibrational energy was significantly higner. This is again in contrast to many one-photon direct dissociations where product distributions reflect initial reactant distributions. Our results suggest that the broad Boltzmann-like product energy distributions we have observed in many IRMPD reactions are not related to the initial thermal energy of the reactant.

During the coming year this apparatus, incorporating $0.01 \mathrm{~cm}^{-1}$ Iaser resolution and molecular beam source, will be employed to explore tne infrarec photodisscciation dynamics of low lying viorational levels o" van der Naals molecules such as ethylene-nitric oxide. 


\section{E. Matrix Isolation Spectroscopy}

(M. E. Jacox)

\section{Fluorine-Atom Reactions}

Studies of the vibrational spectra of the primary products of the reaction of $F$ atoms with small molecules, trapped in solid argon at 14 $K$, have continued, yielding not only the spectra of reaction intermediates but also previously inaccessible information on the mechanisms of these chemical reactions. A review paper entitled "Spectroscopy and Photochemistry of Free Radicals Formed by the Reaction of F Atcms with Small Molecules" and summarizing the results of these and other recent F-atom reaction studies is being prepared for submission to Reviews of Chemical Intermediates.

A series of experiments on the reaction of $\mathrm{F}$ atoms with $\mathrm{CH}_{2} \mathrm{~F}_{2}$ and $\mathrm{HCF}_{3}$ has been completed. Prominent infrared absorptions of $\mathrm{HF}, \mathrm{HCF}_{2}$, and of the $\mathrm{HCF}_{2} \cdots \mathrm{HF}$ hydrogen-bonded complex resulted in the $\mathrm{CH}_{2} \mathrm{~F}_{2}+\mathrm{F}$ studies. The absorptions of $D_{C F} \cdots D E$ grew substantially in intensity when $C_{2} F_{2}+E$ samples were exposed to radiation of wavelength shorter than $300 \mathrm{~nm}$, but the spectra characteristic of $\mathrm{CH}_{2} \mathrm{~F}_{2}+\mathrm{F}$ samples were unchanged on sample irradiation. This observation suggests the formation of a weak $\mathrm{CH}_{2} \mathrm{~F}_{2} \cdots \mathrm{F}$ complex which decomposes by a tunneling mechanism. Moderate yields of $\mathrm{CF}_{3}$ and $\mathrm{HF}$ resulted in the $\mathrm{HCF}_{3}+\mathrm{F}$ experiments, but there was very little evidence for the reaction of $F$ atoms with $\mathrm{DCF}_{3}$. Such isotopic selectivity is consistent with the occurrence of tunneling through a small potential barrier to the reaction. A paper presenting the results of these experiments is in preparation.

A detailed series of experiments on the $\mathrm{F}+\mathrm{CH}_{3} \mathrm{CN}$ system, including isotopic substitution, concentration dependence, and photodecomposition threshold studies, has been completed. Reaction has been found to occur much more rapidly than had been believed on the basis of the detection of the chemiluminescence of the HF product. Both H-atom abstraction and F-atom addition at the central carbon atom have been found to be important. Evidence for the addition of the $F$ atom at the terminal nitrogen atom in the primary reaction was inconclusive, although the infrared spectrum of a product which possesses an NF bond was identified. Although infrared absorptions of isolated HF implied the stabilization of $\mathrm{H}_{2} \mathrm{CCN}$, the infrared absorptions of this radical product could not be positively assigned. The diffusion of $F$ atoms through the solid also leads to the formation of the hydrogen-bonded complex of $\mathrm{HF}$ with $\mathrm{H}_{2} \mathrm{CCN}$. The behavior of the infrared absorptions of this hydrogen-bonded if demonstrated that $\mathrm{H}_{2} \mathrm{CCN}$ is photolytically stable out to $250 \mathrm{~nm}$ and that the strength of the hydrogen bond is similar to that of the $\mathrm{CH}_{3} \mathrm{CN} \cdots \mathrm{HF}$ complex. The infrared absorptions of the addition product, $\mathrm{CH}_{3} \mathrm{CF}=\mathrm{N}$, were sufficiently prominent to permit an almost complete vibrational assignment for this species, which was found to have a CF bond of typical strength and to have a photodecomposition threshold near $280 \mathrm{~nm}$. A manuscript presenting these results is in preparation. 
During the coming year, it is planned to complete a series of experiments on the reaction of $\mathrm{F}$ atoms with $\mathrm{CH}_{3} \mathrm{I}$, in which evidence has been obtained for both the stabilization of $\mathrm{CH}_{3} \mathrm{IF}$ and of $\mathrm{H}_{2} \mathrm{CI}+\mathrm{HF}$. Another important system to be studied is the $F+\mathrm{HCOOH}$ reaction, in which infrared spectroscopic evidence may be obtained for the $\mathrm{H}-\mathrm{CO}_{2}$ intermediates, as well as for cis- and trans-HOCO, previously identified in this laboratory in studies of the reaction of $\mathrm{OH}$ with a $\mathrm{CO}$ matrix. Other systems which will be of particular interest during the coming year are $\mathrm{F}+\mathrm{NH}_{3}$ and $\mathrm{F}+\mathrm{SiH}_{4}$.

With the help of Thomas Buckley, Chemical Thermodynamics Division, hardware interfacing of a microcomputer to the Ebert scanning monochromator used for ultraviolet absorption spectral observations has been completed, and software development is in progress. Priority will be given to studies of the electronic spectra of the products of the reaction of $\mathrm{F}$ atoms with $\mathrm{CH}_{3} \mathrm{NO}_{2}, \mathrm{CH}_{3} \mathrm{CHO}, \mathrm{CH}_{3} \mathrm{CN}$, and $\mathrm{CH}_{3} \mathrm{SH}$.

\section{Excited Rare Gas Atom Reactions}

Preliminary studies have suggested that a small concentration of $\mathrm{CH}_{2}$ may be stabilized when the products of collisional interaction between excited argon atoms and methyl iodide are frozen in an argon matrix. The availability of partially deuterium-substituted methyl iodide may permit the first infrared spectroscopic identification of CHD. Because of the weakness of the product absorptions, the high sensitivity of the Bomem interfercmeter (see Section E) will be important to the completion of these studies, deferred from the previous year because of the pressure of other commitments and the need to complete tests of the performance of the Bomem interferometer under high resolution conditions.

In experiments reported during the past year in which a beam of discharged neon was codeposited with an $\mathrm{Ar}: \mathrm{CF}_{3} \mathrm{NNCF}_{3}$ sample, the most prominent product absorption was that of $\mathrm{CF}_{3}$, with weaker absorptions of $\mathrm{CF}_{3}$ and of an unidentified product, possibly the requisite negatively charged species which maintains overall charge neutrality of the sample. This study provides an important test of the feasibility of detecting the infrared and electronic spectra of molecular ions produced by codepositing a beam of excited neon atoms with a suitable precursor molecule isolated in solid neon. The energy range accessible using excited neon atoms (16.6-16.8 eV) will substantially broaden the range of molecules whose ions are accessible over that covered in an earlier series of experiments in this laboratory using excited argon atoms, permitiing a search for such important ions as $\mathrm{HCO}^{+}, \mathrm{H}_{2} \mathrm{O}^{+}, \mathrm{NH}_{3}^{+}$, and $\mathrm{CH}_{4}^{+}$. The transition to $\exists$ neon matrix will permit the use of a larger pinhole, minimizing wall deactivation of excited neon atoms as they exit ircm the cischarge tube and enhancing the product yield. The use of a neon matrix will also Eliminate spectral perturbations due to charge transfer interaction between molecular cations and the matrix, important in solid arzon. In order to pursue neon matrix studies, a continuous-transfer liquid helium cell (Helitran) has been acquired, and construction of a systen to permit its use with the Bomem interferometer is in progress. The high sensitivity of this instrument will further ennance the pcssibility of 
detecting vibrational and low-lying electronic transitions of molecular ions. During the coming year, experiments should proceed, with high priority to be given to the search for the molecular ions already cited.

3. Vibrational Energy Levels of Transient Molecules

Several months of intensive work were necessary in order to complete and prepare for publication the comprehensive compilation of the vibrational energy levels of ground-state transient molecules possessing from 3 to 16 atoms. This conpilation, which includes almost 500 molecules, will appear in the Journal of Physical and Chemical Reference Data later in 1984. It is planned to maintain an up-to-date digital record of this material and to publish periodic supplements, in order to assist studies of the chemistry and spectroscopy of transient molecules throughout the scientisic community.

\section{Vibrational Fundamentals of Ground-State Diatomic Molecules}

Although the spectra of diatomic molecules have been more exhaustively studied than have been those of more complicated molecules, even for diatomics there are many gaps in our knowledge of the ground-state vibrational frequencies. These have frequently been reported in inert matrices but not in the gas phase. A systematic comparison of gas-phase and inert matrix data is needed. Under conditions in which the matrix shifts are relatively small, matrix data can aid in the assignment of emission spectra and in the development of infrared laser probes, as well as provide a test for the validity of various approximations in ab initio calculations. Approximately 225 pairs of gas-phase and rare gas or nitrogen matrix observations of ground-state vibrational frequencies of diatomic molecules spanning the entire Periodic Table have provided a consistent sample for such a comparison. Conclusions of this study are:

(1) Matrix shifts tend to increase in the order $\mathrm{Ne}<\mathrm{Ar}<\mathrm{Kr}<\mathrm{Xe}<$ $\mathrm{N}_{2}$.

(2) Group Ia and IIIa halides have large red shifts, which may be correlated with the relatively low ionization potential of the Group Ia and IIIa metals. (Very few data are available for compounds of calcium, strontium, and barium, which also have relatively low ionization potentials.)

(3) The one intra-ionic vibration observed, that of $\mathrm{C}_{2}^{-}$, behaves normally.

(4) van der Waals molecules have large blue shifts.

(5) Most other diatcmic molecules--even heavy-metal oxides and fluorides-have argon matrix shifts of less than 2 percent.

The results of this survey are being prepared for publication. 
5. Reaction Intermediates in the Decomposition of Energetic Materials

With support from the Army Research Office and from the Office of Naval Research, a series of experiments has been conducted to determine the role of free radicals in the first stage of the decomposition of energetic materials (e.g., propellants, explosives). The objectives of these studies, for which support terminated at the end of March, included the identification of free radicals formed in the decomposition of such materials, a task to which matrix isolation observations are especially well suited, and the probing of their energy distributions using the techniques of gas-phase laser spectroscopy.

During the three-year pericd of this support, emphasis was placed on determining processes which are important in the initial stages of the decomposition of nitromethane and methyl nitrite. A key study in this project has been that of the photodecomposition of isotopically substituted nitromethane in an argon matrix, completed and published during this past year. An earlier study (1958) of this system, with much less extensive isotopic substitution, suggested that the photodecomposition products in a matrix environment might differ from the gas-phase products. However, several prominent absorptions in that study remained unassigned, and two peaks which were initially believed to have been contributed by HNO have since been found to be contributed by other, unidentified products. In the early stages of the photolysis, infrared absorptions of cis- and trans-CH $\mathrm{CHO}_{3} \mathrm{Orew}$ in intensity. On somewhat longer photolysis, these absorptions diminished in intensity, and there was continued growth in the infrared absorptions now assigned to the $\mathrm{H}_{2} \mathrm{CO} \cdots$ HNO hydrogen-bonded complex. Most of the previously unidentified peaks, as well as the two peaks previously attributed to HNO, continued to grow at this stage of the experiment. These peaks can now be assigned to the cis- and transrotamers of the nitrosomethanol, recently discovered by Dr. R. P. Muller and Prof. J. R. Huber at the University of Zurich. (In October, Dr. M. E. Jacox visited their laboratory to discuss this reassigmment and other topics of mutual scientific interest.) In the later stages of the photolysis, absorptions of $\mathrm{CO}$, NO, and HNCO and of the $\mathrm{H}_{2} \mathrm{O} \cdots$ HNCO hydrogen-bonded complex became increasingly prominent. When the enhanced role of cage recombination in the argon matrix is taken into account, the overall decomposition mechanism is compatible with that determined from gas-phase studies of the photolysis of nitromethane. Because the high probability of cage recombination and of secondary decomposition characteristic of the argon-matrix photolysis experiments is common to the high pressure and condensed phase decomposition of nitromethane, the matrix resulis are of more genera? importance.

Support has been received frcm the Army Research Office for continuaticn and extension of the matrix isolation observations. During the term of this new support, emphasis will ce placed on the infrared and uItrav:0let spectroscopic characterization of reaction intermediates derived ircm the decomposition of benzene and nitrobenzene, in order to provide a 1 irm base for later studies of more complicated arcmatic molecules. Spectroscopic identifications will be sought for a number of other free radical 3. 
including $\mathrm{H}_{2} \mathrm{CN}, \mathrm{CH}_{3} \mathrm{~N},\left(\mathrm{CH}_{3}\right)_{2} \mathrm{~N}$, and $\mathrm{HCN}_{2}$, which also play important roles in the decomposition of explosive or propellant molecules. During the coming year, attempts will be made to obtain a more complete infrared and ultraviolet spectroscopic characterization of the phenyl and phenoxy radicals and to obtain the infrared and ultraviolet absorption spectra of $\mathrm{H}_{2} \mathrm{CN}$, a potential intermediate in the decomposition of RDX and HMX, and of $\mathrm{HCN}_{2}$, believed to be important not only in the decomposition and combustion of energetic materials but also in systems as diverse as the chemistry of the Titan atmosphere and the formation of "prompt" NO in combustion.

\section{F. Future Plans}

There are many experiments planned in the area of vibrational relaxation of molecules in condensed phases. The rates and pathways of energy transfer in molecules on surfaces and in ordinary liquids are not well known. Through our experiments, we hope to understand how the rates are related to structural molecular parameters such as vibrational frequencies, bond lengths and orientations, permanent and transition multipole moments, etc. VET rates for many molecules on metal oxide surfaces will be measured. For instance, on $\mathrm{SiO}_{2}$ surfaces the relaxation of groups such as $\mathrm{OD}, \mathrm{OCH}_{3}, \mathrm{NH}_{2}$ bound to surface $\mathrm{Si}$ atoms will be studied. Other metal oxide substrates such as $\mathrm{TiO}_{2}, \mathrm{ZnO}, \mathrm{BeO}$, and $\mathrm{Al}_{2} \mathrm{O}_{3}$ appear to be suitable for study with our present IR/IR or IR/SPARS methods. Crystalline systems like $\mathrm{OH}^{-}$in the conduction plane of $\beta$-aluminas may permit better definition of bond lengths, angles, and substrate vibrations than the amorphous materials studied so far. We will try to measure VET rates for molecules bound to conducting metals. Metal sols (e.g., Ag, Ar, Rh) adsorb certain molecules (e.g., pyridine) so that infrared and Raman spectra characteristic of surface bound (as opposed to bulk/solution) molecules are prominent, and such samples may permit VET rates at the metal surfaces to be measured with our present apparatus. Alternatively, metal clusters (e.8., 10-30ß diameter) supported on dielectrics (e.g., Pt on $n$ alumina) adsorb many simple species, and offer the high surface area required for our present experimental apparatus to work. However, success in any of these experiments cannot be guaranteed. We note our earlier failures to measure VET rates in molecules bound to dispersed supported metals (i.e., $\mathrm{CH}_{3} \mathrm{NC}$ bound to isolated $\mathrm{Rh}$ atoms on $\mathrm{Al}_{2} \mathrm{O}_{3}$ ). We will try to directly determine where the vibrational energy goes during the relaxation process by measuring the time dependent excitation in different vibrational modes. Either IR absorption spectroscopy using a second independently tunable psec probe pulse (e.8., pump one of the $\mathrm{NH}$ donds in $\mathrm{Si}-\mathrm{NH}_{2}$, probe the other), or IR/SPARS may be used.

A better understanding of the relation between energy transfer and the observed vibrational spectra of molecules in condensed phases (e.g., on surfaces) is desirable. Our initial result for the relaxation of $\mathrm{OH}$ $(\mathrm{v}=1)$ on $\mathrm{SiO}_{2}$ showed that one would have underestimated the $\mathrm{T}_{1}$ time by 2000 if the observed linewidth had been identified as $T_{1}$ broadening. For all our samples we would like to measure the spectrum (IR, Raman), the vibrational relaxation time, and the homogeneous linewidtin $\left(\mathrm{T}_{2}{ }^{-1}\right)$. We may 
estimate the latter through a picosecond CARS experiment using our synchronousiy pumped tunable dye lasers, or perhaps with an IR pump-probe hole-burning experiment.

Although the preceding discussion has focussed on the kinetics of surface-bound molecules, there is also very little known about energy plow in molecules in solution, or in large isolated (gas phase) molecules. For instance there are no obvious experiments in any phase which are comparable to our initial results for $\mathrm{OH}$ on $\mathrm{SiO}_{2}$. We expect to measure VET rates and pathways in certain molecules in solution with the IR/SPARS approach that we used earlier to study the relaxation of $\mathrm{HCl}(\mathrm{v}=1)$. Molecules that may help us understand the surface VET results, e.g., molecules with vibrational frequencies comparable to the substrate modes, will have top priority.

Several years ago we undertook a joint research project involving staff and expertise of the Molecular Spectroscopy and Surface Science Divisions of the Center for Chemical Physics to examine in greater detail molecular dynamics at the gas-surface interface. The thrust of this program was to use laser based, state and velocity selective diagnostics to study molecules being thermally desorbed frcm well-characterized single crystal metal surfaces in an ultra high vacuum environment. The result of our work on NO/Ru(001) has been published and reviewed herein in past years. We are currently performing measurements on the No/Pt(111) system. Our preliminary results indicate significant differences in the molecular dynamics important in even this simple bond breaking process for the flat, basal planes of Pt vs Ru. What is important about these experiments is that they are extremely, perhaps uniquely, sensitive to the interactions between gas molecules and different metallic surfaces.

The future directions of this research effort can be broken up into two branches. First, in view of the marked differences for NO/Pt vs Ru we shall explore thermal desorption as a means of studying the dynamics of related systems. We shall probe No desorption from surfaces such as Pt where there is only simple molecular desorption, from Ru where decompcsition competes with desorption and Ir which exhibits aton-aton recombination, leading to the desorption of No. Similarly we shail look at gaseous partners exhibiting different chemistries such as $\mathrm{CO}$ and $\mathrm{H}_{2}$. These studies are only now becoming practical with the advances being made on vacuum ultraviolet laser development. Potassium has shown to be an important promoter in hydrocarbon chemistry. It has also been observed that small amounts of $K$ induces large shifts in the thermal desorption spectra for CO from Ni. This is one system we hope to follow to explore the role of reaction promoters or inhibitors.

Thermal desorption studies are sensitive to the molecular dyanics and energy flow in the region of the top of the chemisorption potential energy well - a region important in the making and breaking of chemica? bonds. In the thermal desorption experiment the heating rates traditionally used are much slower $(e .8 . i 0 \mathrm{~K} / \mathrm{sec}$ ) than other molecular relaxation processes which is good if one's goal is to study the dynamics of a h!gh!y 
energetic system under "equilibrium" conditions. However, it is impossible under these conditions to learn about energy transfer at lower levels of molecular excitation at clean metallic surfaces. The second branch of our project will use rapid pulse laser heating to directly desorb transient species at metal surfaces. Specifically, we now have available tunable picosecond lasers (developed through the Picosecond Canpetences Program) which will enable us to vibrationally excite adsorbate species such as $\mathrm{NO}, \mathrm{CO}, \mathrm{OH}$, etc.to their $\mathrm{v}=1$ or 2 states. At some variable time delay (i.e., 1 to 100 ps) a second intense, non-resonant picosecond pulse will heat the surface (at a rate of $-10^{14} \mathrm{~K} / \mathrm{sec}$ ) to a temperature of -1000 $K$ from which the adsorbate will be directly desorbed. Those surviving in the $v=1$ state during the time period $\Delta t$ between pump and heating laser pulses will be defected by laser fluorescence or ionization techniques in the gas phase as they leave the surface. These experiments will provide the first direct measurements of vibrational lifetime of adsorbates on single crystal metals. Determination of the rates and mechanisms for energy transfer at these low levels are important for understanding heterogeneous reactions and the role of catalysts or reaction promoters/inhibitors in such processes. Although many spectroscopic studies of adsorbates on metals exhibit linewidths consistent with sub-picosecond uncertainty broadening our results for $\mathrm{OH}$ on the surface of $\mathrm{SiO}_{2}$ show that vibrational relaxation rates may be much slower than that predicted from linewidth analysis. The proposed rapid desorption technique should allow the successful extension of their work into the realm of ordered metallic surfaces.

The completion of the matrix isolation setup for use with the Bomem interferometer will open up new vistas for studies of reaction intermediates. The much improved sensitivity of this instrument will make possible the study of weak product absorptions, previously below the threshold for detection. Two important classes of molecule--carbon-13 substituted molecules present in natural abundance and products of 0-atom reactions in discharge sampling experiments--will become accessible under these conditions. Observations of the carbon-13 species will aid in product identification and will provide valuable data for infrared spectral analysis. Since water and oxygen impurities generally lead to the formation of 0 atoms in discharge sampling experiments, the greater sensitivity of this interferometer will both facilitate and mandate a series of studies of the reactions of 0 atoms with small molecules. Studies of the products of the reaction of 0 atoms with benzene and with nitrobenzene form an important part of the current program of research being supported by the Army Research Office. The interferometer also will provide access to the $4000-12,000 \mathrm{~cm}^{-1}$ spectral region, for the irst time permitting study of the near infrared absorption spectra of such important free radicals as $\mathrm{C}_{2}^{-}, \mathrm{HC}_{2}$, and $\mathrm{HO}_{2}$ in an inert matrix environment.

Empinasis will be placed on the studies of the interaction of excited neon atoms with small molecules, to be implemented during FY 85 . It is hoped that these studies will provide much previously inaccessible information on the vibrational and electronic spectra of molecular ions, a topic of great current scientific interest. Since the study of F-atom 
reactions has proved to be an extremely versatile tool, it will continue, but with increasing emphasis on the identification of the electronic spectra of free radicals formed in $\mathrm{F}$-atom reaction systems.

The need for more complete characterization of the energy levels of new free radicais and molecular ions stabilized in studies of the reactions of $O$ and $F$ atoms and of excited neon atoms with small molecules will mandate the construction of a laser-excited fluorescence setup for matrix isolation studies. Recent improvements in laser sources of ultraviolet radiation will make possible the detection of previously inaccessible low-lying molecular electronic states. Fluorescence and phosphorescence may result when these states are pumped by cage recombination of the molecular fragments formed on laser photodissociation of the matrix-isolated molecule.

The compilation of the ground-state vibrational energy levels of transient molecules will be kept current, and from time to time supplements will be issued. It is also planned to initiate a compilation of excited electronic states and their vibrational energy levels for transient molecules. 


\section{QUANTUM CHEMISTRY GROUP}

M. Krauss, H. Basch, P. S. Julienne, F. H. Mies,

M. Rosenkrantz, S. B. Sears, and W. J. Stevens

\section{A. Introduction}

Atomic and molecular electronic structure and scattering calculations are applied to chemical and spectroscopic problems where experiments are difficult to perform or so complicated that the theory is needed to model the results. The electronic structure problems are now drawn from biotechnology areas. Quantum chemistry methods are being applied to energetic, conformational, and spectroscopic approacnes to biochemical problems. Applications of scattering theory have been made to areas such as high temperature materials, photodissociation of high temperature species, and pressure broadening and depolarization of atomic radiation. The summary of activities for the past year is divided into two areas: electronic structure and scattering. Several collaborative activities with other groups were undertaken during the past year. These are described in sections B. $1 . \mathrm{b}, 3-6$ and $C .6,7$.

B. Electronic Structure

(M. Krauss, W. J. Stevens, H. Basch (NBS Research Associate)

1. Biomolecule Calculations

Fundamental studies are underway on the electronic structure at active sites of enzymes and for the determination of the interaction energies and spectroscopic behavior of metal complexes interacting with DNA and RNA by using suitably chosen model systems. Ab initio quantum mechanical techniques are used and relativistic effective potential methods permit the calculation of the electronic structure and properties of metalloorganic molecules with metals from anywhere in the periodic table.

\section{a. Compact Effective Potentials}

Compact effective potentials (CEP) and their concomitant expansion bases have been developed to reduce the cost of ab initio molecular orbital calculations and make the inclusion of heavy metals reasible. It is now possible to carry out double zeta (or better) basis set calculations on large biomolecule fragments. This is necessary since the interactions between such fragments are modelled from their charge distributions which, up till now, have been obtained with small, possibly inaccurate, basis sets. The polarization of the fragments is also found to be important and it is possible that this effect is underrated because the small basis set calculations are not flexible enough to account for the magnitude of the polarization. 
Excellent comparisons between CEP and all-electron single and multi-configuration self-consistent-field calculations have been obtained for a variety of molecules in the first two rows of the periodic table for both binding energies and spectroscopic constants. Relativistic potentials are being derived for all elements beyond the second row, and the spin-orbit potential is also being obtained. To date only selected metal potentials have been considered as needed, but we plan to construct potentials for the entire periodic table. A paper describing the algorithm for generating the potentials and tabulations for the first tio rows of the periodic table has been prepared.

\section{b. Binding of Pt complexes to DNA \\ Binding energies of the $\mathrm{P} \leftarrow\left(\mathrm{NH}_{3}\right) 3_{3}^{2+}$ complex to the bases,} $\mathrm{H}_{2} \mathrm{O}, \mathrm{NH}_{3}$, imidazole, pyrimidine, 2-pyrimidone, 4-pyrimidone, guanine, cytosine, adenine, and thymine, were calculated using the CEP at the SCF level. The results show that the apparent binding to the different sites in the DNA bases is likely to be determined by enthalpic and not kinetic effects. The binding is much larger to guanine and cystosine sites than to the other bases.

Experimentally it is known that the platinum complex forms covalent, and often bifunctional bonds with guanine, cytosine, and adenine. The preferred binding has been deduced experimentally to be intrastrand and primarily to guanine bases. However, little is known about the strength of the binding to various sites, whether the binding preference is determined by thermodynamic or kinetic factors, or what disruption of the DNA structure occurs in order to accommodate the complex. Large-scale quantum mechanical self-consistent-field calculations were undertaken to quantify the binding energies of platinum ammonia complexes to the various base ligands listed above. Since platinum forms four-coordinate square planar complexes, a triamine complex, Pt $\left(\mathrm{NH}_{3}\right)_{2}^{+}$, was used to examine the binding to the individual bases. +2 For exampIe, Figure 4.1 shows the oreferred orientation of $\mathrm{Pt}\left(\mathrm{NH}_{3}\right)_{3}^{+2}$ interacting along the lone pain of $\mathrm{N} 7$ in guanire.

The stability order for binding to varicus sites in the free bases is calculated to be $N 7(G)>N 3(C)>02(C)>06(G)>N 3(A)>N 7(A)>04(I)>$ $02(T)$. Binding $N 7(G)$ is favored by $10-15 \mathrm{Kcal} / \mathrm{mole}$ over $\mathrm{N} 3(\mathrm{C}), 02(\mathrm{C})$ and $06(G)$ and by more than $30 \mathrm{Kcal} / \mathrm{mole}$ over sizes in adenine and thymine. Calcuiations of the optimum Pt-base ligand distances and the energy variation as a furction of bending the molecular plane of the bases were determined in order to characterize the essential features of the interaction enerzy surface. 


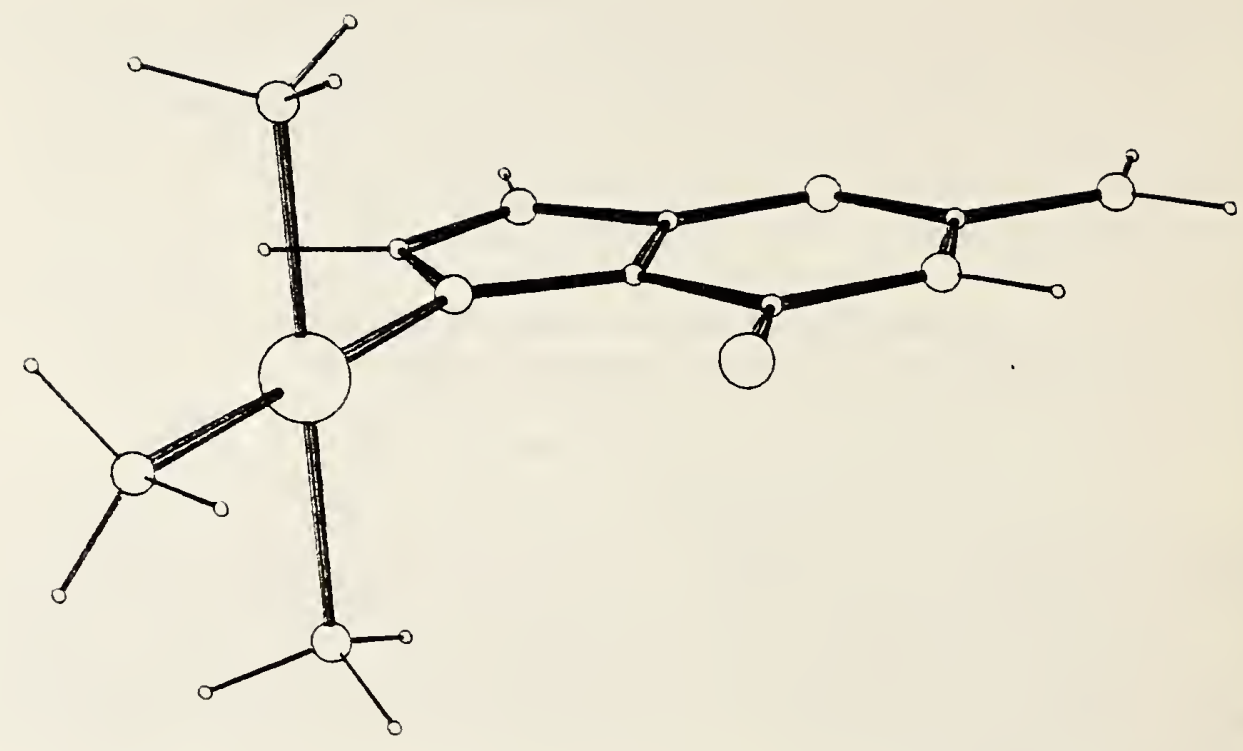

Fig. 4.1. Preferred orientation of the $\mathrm{Pt}\left(\mathrm{NH}_{3}\right)_{3}^{+2}$ complex interacting with
guanine.

The quantum mechanically determined binding energies and potential surface features were used to develop model DNA structures which could accommodate intrastrand binding of $\mathrm{Pt}\left(\mathrm{NH}_{3}\right)_{2}^{+2}$ to adjacent bases. Molecular mechanical modeling of DNA was carried out in collaboration with Prof. $K$. J. Miller and E. R. Taylor of the Renssalaer Polytechnic Institute Department of Chemistry. Principal findings include a low-energy kinked structure of poly $(d G) \cdot p o l y(d C)$, as shown in Figure 4.2, which can easily accommodate binding of the platinum complex to adjacent guanines in a nearly optimal conformation in the major groove. In addition, a novel binding site in poly (dGpdC) has been proposed in which ar anti $\rightarrow$ syn rotation of cytosine allows the binding of the platinum ccmplex between $N 7(G)$ and and N3(C) on adjacent bases. Several other structures, including binding to non-adjacent base pairs and binding in the minor groove, were examined and found to be energetically unfavorable.

c. Energetics and Conformations of cis and trans Pt $\left(\mathrm{NH}_{3}\right)_{2} \mathrm{AB}$ ( $A, B=\mathrm{Cl}$ or $\mathrm{A}=\mathrm{NH}_{3}, \mathrm{H}_{2} \mathrm{O}, \mathrm{OH}^{-} ; \mathrm{B}=\mathrm{H}_{2} \mathrm{O}, \mathrm{OH}^{-}$).

These studies were undertaken to learn more about the drug compounds that directly interact with DNA since little is known experimentally about the $\mathrm{H}_{2} \mathrm{O}$ and $\mathrm{OH}^{-}$systems. Using energy gradient methods, the isomeric energies and conformations were calculated for all 

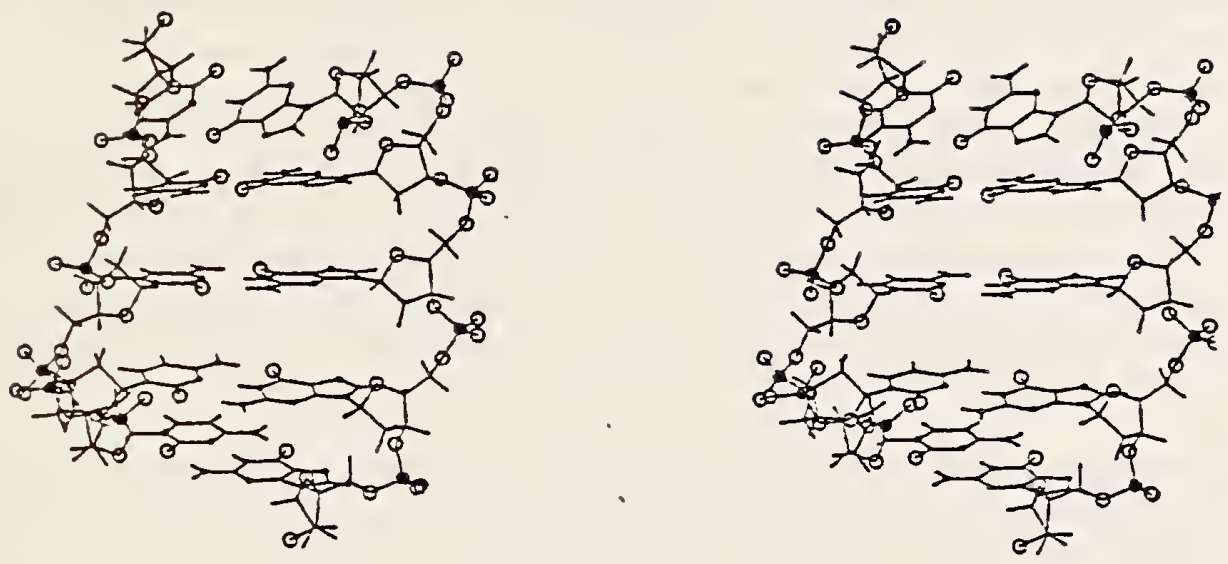

Eig. 4.2. Stereo image of poly $(d G) \cdot \operatorname{poly}(d C)$ kinked by $40^{\circ}$ to allow pt-complex binding in the major groove.

the molecules. The energy surface for cis-trans isomerization for the $\mathrm{Pt}\left(\mathrm{NH}_{3}\right)_{2}^{+2}$ complex was also examined. The calculated geometries were used to quantify the 'trans influence' ligand ordering. The variation in bond lengths as a function of trans substitutions produced an order $\mathrm{OH}^{-}>\mathrm{Cl}>$ $\mathrm{NH}_{3}>\mathrm{H}_{2} \mathrm{O}$. This ordering agrees with the conventional one except for $\mathrm{OH}^{-}$ which is usually considered to be similar to $\mathrm{H}_{2} \mathrm{O}$. The discrepancy is probably connected with the difficulty in experimentally characterizing hydroxo complexes.

Strong hydrogen bonds are obtained with the ionic lizands for favorable geometric configurations. When these $\mathrm{H}$-jonds are formed, the energy difference between the hydroxy containing isomers is very small.

d. Cation Effect on H-bonds and Tautomeric Energies

In the local vicinity of the cation (bare or complex), the binding energies and conformations of nearby hydrogen bonds and the proton or substituent positions can be suostantialiy altered. A set of modeI calculations on $H$ bonding effects was done for a pair of hydrogen-bonded imidazoles. Binding energies, equilibrilum bond lengths, and barrier heights are all substantially affected by the perturbations. Detailed analysis of the perturbations in the charge distributions due to cation binding is still underway. 
2. Electronic Structure of Metal Oxides

(M. Krauss and W. J. Stevens)

This work represents the completion of research supported by the Air Force Office of Scientific Research. Complete active space (CAS) multi-configuration self-consistent-field (MC-SCF) calculations were performed for 12 valence electrons distributed among the sigma, two pi, and one delta molecular orbitals for a large number of electronic states of FeO and RuO. Spectroscopic constants and spin-orbit coupling constants were evaluated and the results used to analyze binding in transition metal oxides. The accurate spin-orbit coupling constants show that the CAS-MC-SCF wave functions give a qualitatively correct description of the electronic structure but the spectroscopic constants are far more accurate for the Ruo states than for FeO.

Calcuiations on the comparative behavior of $\mathrm{Nd}$ and $\mathrm{U}$ oxides is still continuing. Of particular interest is the calculation of $\mathrm{sf}^{3}(5 \mathrm{I})$ and $s f^{2} d(5 K)$ configurations (states) of almost the same energy for the ground state of UO. In the case of $\mathrm{NCO}$ the $\mathrm{sf}^{2} \mathrm{~d}$ configurations are considerably higher in energy than the sf $^{3}$. The comparable ion configurations have also been calculated and intermediate spin-orbit coupling has been included in the analysis of the ions.

3. $\mathrm{Ab}$ initio study of $\mathrm{Mn}^{+}$Reacting with $\mathrm{H}_{2}$ (with M. Dupuis and $W$. Lester, Lawrence Berkeley Laboratory)

Using the ion beam mass spectrometry technique, Armentrout and coworkers ( $U$. of Cal. Berkeley) have measured the bond strengths of atomic metal ions with the species $\mathrm{H}, 0$, and $\mathrm{CH}_{n}(n=0-3)$. Bond energies of the metal hydride ions are obtained from the reaction cross sections for

$$
\mathrm{M}^{+}+\mathrm{H}_{2} \longrightarrow \mathrm{MH}^{+}+\mathrm{H}
$$

A plot of the cross section vs. energy for (1) shows two peaks for $\mathrm{Mn}^{+}$, unlike other metal ions studied, which is attributed to different reactivity of the $7_{\mathrm{S}}$ and ${ }^{5} \mathrm{~S}$ states of $\mathrm{Mn}^{+}$. The ${ }^{7} \mathrm{~S}$ spin configuration is believed to be essentially nonreactive with $\mathrm{H}_{2}$. The ${ }^{5} \mathrm{~S}$ configuration is presumed to account for $\mathrm{MnH}^{+}$.

We have characterized the essential features of the potential energy surfaces which govern the reactions of $\mathrm{Mn}^{+}\left({ }_{\mathrm{S}},{ }_{\mathrm{S}} \mathrm{S}\right)$ with $\mathrm{H}_{2}$. The reactants, transition states, and products have been accurately characterized by ab initio quantum mechanical methods to provide insight on the factors responsible for the different reactivity of the above states of $\mathrm{Mn}^{+}$. Although the analysis is not yet complete, our results do not support the proposed different reactivities of the $7_{S}$ and ${ }^{5}$ states of $\mathrm{Mn}^{+}$. 
The calculations have been carried out with compact effective potentials (CEP) replacing the core electrons on manganese. Test calculations on $\mathrm{MnH}^{+}$using all-electron methods have compared extremely well with the CEP results.

4. Electronic Structure of Low-Lying States of NaK (with D. Konowalow and L. Ratcliff, SUNY Binghamton)

We have calculated the potential energy curves for all molecular states of $\mathrm{NaK}$ which may be obtained from the interactions $\mathrm{Na}(3 \mathrm{~s})+\mathrm{K}(4 \mathrm{~s}), \mathrm{Na}(3 \mathrm{~s})+\mathrm{K}(4 \mathrm{p}), \mathrm{Na}(3 \mathrm{p})+\mathrm{K}(4 \mathrm{~s}), \mathrm{Na}(3 \mathrm{~s})+\mathrm{K}(5 \mathrm{~s}), \mathrm{Na}(3 \mathrm{~s})+\mathrm{K}(3 \mathrm{~d})$, and for the $\Delta$ states corresponding to the interactions $\mathrm{Na}(3 \mathrm{~d})+K(4 \mathrm{~s})$ and $\mathrm{Na}(4 p)+K(4 p)$ by full-valence configuration interaction computations which utilize effective core potentials to describe the core electrons, the core-valence orthogonality constraints, and the core-valence correlation (CVC) energy. The differences between our computed curves and those deduced from experimental spectra are generally small and can be accounted for by: (1) the modest size of the basis set, which is insufficiently diffuse to describe $\mathrm{Na}^{-}$and $\mathrm{K}^{-}$resonances associated with excited charge transfer interactions and related molecular Rydberg character, and (2) the approximate way in which the CVC interaction is included.

These accurate potential curves for the 22 lowest states of Nak will be of considerable use in the analysis of molecular. spectra and molecular beam scattering. A tabulation of dipole transition moments for ali allowed transitions among these states is now in preparation.

5. Effect of Electron Correlation on $\mathrm{e}-\mathrm{N}_{2}$ Scattering (with J. Rumble, NBS and D. Truhlar, U. of Minn.)

Most electron scattering calculations are carried out using Hartree-Fock target wavefunctions. Although this approximation may be valid for target molecules near their equilibrium geometries, electron correlation can be important for vibrationaliy excited targets. To test this possibility, elastic scattering cross sections for electron scattering by $\mathrm{N}_{2}$ have been calculated in the static-plus-local-exchange model using two different target wavefunctions: Hartree Fock (HF) and Multiconfiguration SElf-Consistent-Field First-Order-Conf iguration-Interaction (MCSCF-FOCI). The range of impact energy considered is 1.5 to $30 \mathrm{eV}$. Inclusion of electron correlation in the target wavefunction changes the position of the $\pi_{\mathrm{g}}$ low-energy resonance by about $0.5 \mathrm{eV}$. Nonresonant partial cross sections typically change by 2 percent at the equilibrium internuclear separation but sometimes more (up to 26 percent at the equilibrium internuclear distance and even more at stretched secmetries).

6. Damped Dispersion Interaction Energies

(Ph.D thesis of M. Rosenkrantz, SUNY, Binghamton, directed by M. Krauss)

A theory has been developed for the calculation of the dispersion energy for overlapping polyatomic fragments. A computer code has been written and applied to $\mathrm{ArH}_{2}$, ArHE, and $\mathrm{ArHC}$. The present 
calculations provide the first theoretical data on the anisotropy of the dispersion interaction. Critical analyses of the previous theoretical treatments shows considerable differences which are attributed to the semi-empirical nature of such treatments and suggests a caution in the use of such empirical methods.

\section{c. Scattering}

1. Nonadiabatic Theory of Dissociative Molecular States (P. S. Julienne and F. H. Mies)

Given current access to powerful quantum chemistry codes which provide interaction potentials, electronic wavefunctions and coupling matrix elements for diatomic molecules, and given the very stable numerical close-coupling codes that are available, we can generate multichannel continuum wavefunctions $\Psi_{i}^{+}$for the diatom $A B$ of almost arbitrary accuracy. If we couple these numerical capabilities with the rigorous analytic description of the diatomic wavefunctions provided by multichannel quantum defect analysis (MCQDA), especially in the vicinity of dissociation thresholds, we are in a position to describe a wide variety of diatom phenomena with a great deal of confidence, and with renewed insights.

There are a number of examples of interesting phenomena associated with the interaction of light with colliding atoms, represented by the reaction

$$
[A+B]_{i}+n \hbar \omega_{0} \rightarrow\left[A^{\prime}+B^{\prime}\right]_{f}+(n \pm 1) \hbar \omega_{0}
$$

For example, if the $A^{\prime}+A$ transition is dipole allowed and the perturber does not change state, equation (1) represents ordinary atomic line broadening. Such a collision has been called an optical collision. If the $A^{\prime}+A$ transition is forbidden, (1) represents a collision-induced transition. If both $A$ and $B$ change states, (1) represents a radiative collision, sometimes called LICET. From the viewpoint of molecular spectroscopy these are all simply diatomic continuum-continuum transitions with a radiation scattering matrix element given by

$$
S_{f i}=-2 \pi i \mid\left\langle\psi_{f}^{-} \varepsilon_{0} \hat{e}_{0} \cdot \vec{\mu} \mid \Psi_{i}^{+}\right\rangle \text {. }
$$

Even in this weak-field, linear dipole $\vec{\mu}$, approximation, we require proper multichannel continuum wavefunctions for the $\Psi_{i}^{+}$incoming complex $[A+B]_{i}$ and the $\Psi_{f}^{-}$outgoing collision complex $\left[A^{\prime}+B^{\prime}\right]_{f}{ }^{i}$ in Eq. (1). These incorporate the non-adiabatic effects which determine the internal state distributions of the atomic fragments.

We have developed a close-coupled theory of atomic collisions in the presence of radiation coupling which yields a direct determination of $S_{f i}$ and may be used to calculate the distribution of final atomic states which 
results from absorption of polarized light during a collision. The radiation field is assumed to be weak enough that the transition probabilities remain linear in field intensity $\varepsilon_{0}$. The molecular picture is emphasized in which the wavefunction is expanded in a basis of field-pree molecular states and the hamiltonian is blocked in accordance with molecular quantum numbers.

In addition, using MCQDA, we have shown how Eq. (2) can orten be iactored as follows,

$$
S_{\rho i}=\left(\bar{N}_{\hat{f} \hat{f}}^{-}\right)^{*} S_{\hat{f} \hat{i}}^{B O}\left(N_{\hat{i}}^{+}\right)
$$

the 'incoming' halp-collision matrix $\mathrm{N}_{1}^{+}$tracks the incident collision complex from infinity, where it enters in channel state $\psi_{i}$, into distances near $\hat{R}_{0}$, where the system 'coalesces' into the selected electronic rotational state we designate as $\dot{\psi} \hat{i}$, such that

$$
\phi_{\hat{i}}=\sum_{i}, \psi_{i}, \tilde{m}_{i}, \hat{i}\left(R_{0}\right)
$$

The transformation matrix $\mathrm{m}\left(\mathrm{R}_{0}\right)$ is orthogonal, in $=1^{\circ}$, and depines a molecular state which we postulate is the dominant source of absorption at the prescribed photon frequency. Further, we assume that only a single, photon-selected upper state $\phi_{f}$ is accessed at $R=R_{0}$,

$$
\phi_{\hat{f}}=\sum_{\hat{f}}, \psi_{\rho}, \bar{x}_{f^{\prime}, f}\left(g_{0}\right)
$$

The MCQDA defined 'OLtgoing' halp-collision matrix $\mathrm{N}^{-}$follows the subsequent dissociation of this quasi-molecule as $i t$ proceeds to $\mathrm{R}=\infty$ where the atoms depart into channel $\psi_{f}$. Thus $\mathrm{N}^{+}$and $\mathrm{N}^{-}$isolate and summarize all the inelastic and non-adiabatic coupiing efpects operative among the incident and final channels respectively. These foctors are bridged jy the usual Born-Oppenheimer ( 30 ) matrix element $S$ BO wich approximates the transient total molecular states in the vicinity of ${ }_{0}$ as simple products of an electronic-rotational state and a radial wavefunction.

We have applied the half-coliisicn theory to the analysis of the $\mathrm{Sr}\left({ }^{1} \mathrm{P}\right)+\mathrm{Ar}$ depolarization and ind that we can reproduce much of the close-coupled data with this simple model. First order corrections sugzested by MCQDA brings us into aimost quantitative agreement with the exact cuantal results. 
Many more calculations need to be performed on a variety of systems to fully test the half-collision analysis. However, even if there are occasional quantitative failures, the qualitative insights that are offered give us good direction in choosing parameters for the full quantal calculations. Further, we must explore the applications in other branches of continuum spectroscopy. Note that if we substitute a bound state for the initial continuum state $\psi_{j}^{+}$in $\mathrm{Eq}$. (2) we would obtain a bound-continuum transition matrix element in place of $S_{f i}$ which would involve the same $\mathrm{N}^{-}$ half-collision matrix. Thus our analysis of $\mathrm{N}^{-}$and the implications of unitarity and frame transformations should find equal application to photodissociation (and predissociation).

2. The Thermodynamic Effects of Inelastic Collisions (F. H. Mies)

The accurate evaluation of thermodynamic properties at elevated temperatures requires proper analysis of the dissociated states of molecules. These continuum states make substantial contributions to the molecular partition functions and result in significant modifications of the equation of states and equilibrium constants for high temperature gases. Conventional spectroscopic analysis of the partition functions, as employed in the JANAF Tables, generally tend to overestimate the density of states for diatomic molecules at high energies, and therefore underestimate the degree of dissociation into atomic fragments. Alternately, explicit summations which exclude energy states which lie above the diatom dissociation limit will exaggerate the extent of dissociation.

Rigorous analysis of the molecular partition function can only be derived from the quantum mechanical treatment of the continuum states using molecular scattering theory. These continua are synonymous with the scattering wavefunctions which define the elastic and inelastic collisions experienced by the departing fragments. This approach yields unambiguous interpretations of metastable, or predissociating states, as well as proper descriptions of second virial coefficients and imperfect gas effects at high temperatures. Great calculational simplifications can be achieved using classical phase integrals, but such techniques must first be certified by comparison to rigorous quantum results.

Using formal scattering theory to characterize the continuum wavefunctions associated with molecular fragmentation we derive rigorous expressions for the molecular partition function $Q$. In particular the analysis includes the effect of inelastic collisions which are introduced by the off-diagonal elements of the scattering matrix $\underline{S}(E)$ at each total energy. Expressions are reduced to a simple form using the eigenphases of the associated $Y(E)$ matrix derived from our recent multichannel quantum defect analysis (MCQDA) of molecular dissociation. The partition function $Q=Q_{e l}+Q_{i n e l}$ can be separated into distinct elastic $Q_{e l}$ and inelastic

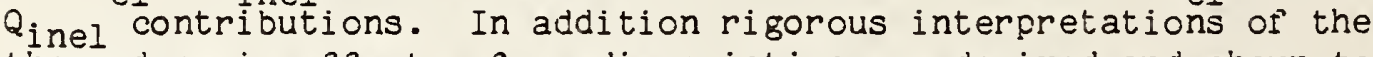
thermodynamic effects of predissociation are derived and shown to have negligible consequences on equilibrium properties. 
Using previously evaluated $Y(E)$ matrix elements, we would have to conclude that the neglect of inelastic coupling in the calculation of thermodynamic functions is entirely reasonable. There are a number of subtleties associated with thresholds that could modify the partition functions, but these effects would probably be of Iimited importance, especially at elevated temperatures.

The biggest limitation on the general validity of our conclusion is that all our numerical evaluations were devoted to simple two-channel couplings. It is possible that there are some fortuitous cancellations that are not present in the more general cases, and it remains for us to explore more complicated inelastic events.

3. Analyses of Pseudopotentials and their Applications to Multichannel Couplings in Atoms

(F. H. Mies and S. B. Sears)

Previously we have calculated norm-conserving pseudopotentials which accurately reproduce the elastic scattering of a Rydberg electron in the static-exchange limit for collisions with both closed $\mathrm{Na}^{+}\left(1 s^{2}, 2 s^{2}, 2 p^{6}\right)$ and open $\mathrm{He}^{+}(1 \mathrm{~s})$ shell ion core states. We have shown that these potentials, obtained from accurate numerical Hartree-Fock atomic wavefunctions for the lower Rydberg states, such as the He(1s,np') 1 p states, can be scaled to obtain reliable representations of the correlated Rydberg wavefunction throughout the higher members of the Rydberg series and into the ionization continuum.

Our present studies are devoted to extending the use of exchange and correlation localized potentials to the inelastic scattering effects associated with the configuration-interaction between various open-shell Rydberg series. Specifically we are using the well studied four channel coupling in the $\mathrm{He}^{+}+e^{-}$system to test our development of appropriate off-diagonal pseudopotentials,

$$
\begin{aligned}
\mathrm{He}^{+}(1 \mathrm{~s})+\mathrm{e}^{-}(2=1) & \rightleftharpoons \mathrm{He}^{+}(2 \mathrm{~s})+\mathrm{e}^{-}(2=1) \\
& \rightleftharpoons \mathrm{He}^{+}(2 \mathrm{p})+\mathrm{e}^{-}(l=0,2) .
\end{aligned}
$$

First, we have derived new pseudopotentials from the ${ }^{1} \mathrm{P}$ states of $\mathrm{He}\left(2 \mathrm{~s}, \mathrm{np} \mathrm{p}^{\prime}\right) \mathrm{He}\left(2 \mathrm{p}, \mathrm{ns}^{\prime}\right)$ and $\mathrm{He}\left(2 \mathrm{p}, \mathrm{nd}^{\prime}\right)$. These, combined with our previously determined He(1s,np') potential gives us an accurate representation of the elastic scattering in each of the four channels which contribute to Eq. (1). We have also obtained the various combinations of configuration

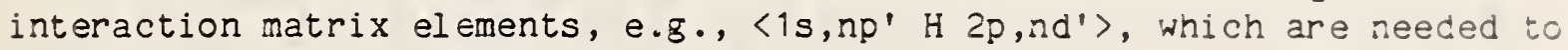
develop our off-diagonal potentials. In addition we have modified our close-coupled scattering codes to handle Coulcmb scattering boundary conditions and have already performed accurate calculations of the inelastic scattering and the autoionization of the core-excited kycber 3 levels in the complete absence of exchange. Jur success in developir.g useful algorithms to handle off-diagonal excharge remains to be iested. 
4. Adiabatic Representations of Distant Perturbations in Molecular Spectroscopy

(F. H. Mies)

We have considered the strong coupling of a given molecular state $\psi_{0}(r, R)$ to an energetically distant state $\psi_{1}(r, R)$ such that the total wavefunction at total energy $E$ must be represented as follows,

$$
|E\rangle=\psi_{0}(r, R) F(E, R)+\psi_{1}(r, R) G(E, R) .
$$

A particular radial coordinate $R$ in the molecular Hamiltonian $H(r, R)$ has been isolated for special consideration, and $r$ is meant to represent the composite of all remaining degrees of freedom. Thus $\psi_{0}$ and $\psi_{1}$ are but two of an infinite set of states which span the space of $r$. We generate a pair of coupled equations for $F$ and $G$ by imposing the condition (H-E) $E>=$ $O$ and operating from the left with $\psi_{0}$ and $\psi_{1}$ followed by an integration over $r$,

$$
\begin{aligned}
& {\left[T=V_{00}(R)-E\right] F+V_{01}(R) G=0} \\
& {\left[T+V_{11}(R)-E\right] G+V_{10}(R) F=0 .}
\end{aligned}
$$

Note that the functions $\psi_{0}$ and $\psi_{1}$ may contain an implicit dependence on $R$ and will not in general commute with the radial kinetic energy operator $T$ $=-\partial^{2} / \partial R^{2}$. Thus the 'potentials' may actually contain momentum operators which are required to operate on the $F$ and $G$ functions. For the present purposes it is adequate to assume that we can neglect such radial Born-Oppenheimer couplings, and thus we can also assume that the off-diagonal coupling $V_{01}=V_{10}$ is both real and symmetric.

We have previously discussed the role of adiabatic electronicrotational (AER) states in diatomic spectroscopy, where $\psi_{0}$ and $\psi_{1}$ represent pure electronic-rotational states constructed in some appropriate Hund's coupling scheme. Further we have already made explicit application of AER theory to describe the effects of distant perturbations in atomic scattering. In that case $E$ is continuous since it exceeds the dissociation energy limit $E>V_{O O}(\infty)$. Here we want to explicitly apply AER theory to pure bound state spectroscopy where $E<V_{0 O}(\infty)$ and both channels are closed. Also we have relaxed any restriction to diatomic molecules and have applied AER theory to vibronic coupling in polyatomic systems.

Our major concern is the applicability of AER theory to strongly perturbed systems. For this purpose we consider the strong vibronic couplings and resultant intensity borrowings analyzed by Innes and others for systems such as formaldehyde and propynal. As implied in Eq. (1) we are only allowing a single active vibrational mode, with displacement $R$, to couple the two states. Both $\psi_{0}$ and $\psi_{1}$ now contain a common set of frozen vibrational wavefunctions with identical quantum numbers and we 
have merely treated the polyatom as a pseudo-diatom. We could of course unfreeze two coordinates, say $R_{1}$ and $R_{2}$, and postulate a set of interaction potentials $V_{00}\left(R_{1}, R_{2}\right), V_{11}\left(R_{1}, R_{2}\right)$ and $V_{01}\left(R_{1}, R_{2}\right)$, together with a pair of kinetic energy operators $T_{1}\left(R_{1}\right)$ and $T_{2}\left(R_{2}\right)$. This would give rise to the Duschinsky effect and we believe the AER theory could be applied with equal force to this problem. However, we shall only consider the simple one-mode coupling represented by Eqs. (1), (2), and (3).

In the absence of any $V_{01}$ coupling we woufd simply replace Eq. (1) with a pure Born-Oppenheimer wavefunction $\psi_{0} \cdot f_{n}^{0}\left(E_{O n}, R\right)$ where $f_{n}$ belongs to the set of diabatic vibrational wavefunctions generated by ${ }^{n_{O O}}$ at the unperturbed eigenvalue $\mathrm{E}_{\text {On }}$,

$$
\left(T+V_{00}\right) f_{n}^{0}=E_{0 n} f_{n}^{0}
$$

If the energy is scaled such that the zero-point energy $E_{00}$ of the $n=0$ ground vibrational level exactly equals $1 / 2$, and if we approximate $V_{00}=$ $R^{2} / 4$ as a pure harmonic potential the unperturbed eigenvalues are located at $E_{O n}=(n+1 / 2)$. The levels, and the associated quantum numbers are displayed on curve (a) of Fig. 4.3. These levels are perturbed by a state with an identical harmonic potential $V_{11}=R^{2} / 4+\Delta$ which is merely displaced by a constant energy

$$
\Delta=V_{11}-V_{00}
$$

This simple model is not a necessary ingredient in the adiabatic theory but does expedite the evaluation of the exact wavefunctions and eigenvalues that we require to compare to the AER results. For the propynal potential (curve b) $\Delta=25.313$, while the formaldehyce potential (curve c) was simulated with $\Delta=24.920$. The associated of $f$-diagonal coupling is taken to be of the form $\mathrm{V}_{01}=\lambda$. with $\lambda=2.1197$ and $\lambda=$ 2.7890 for curves (b) and (c) respectively. These curves represent the lower adiabatic potential given by

$$
V_{\alpha \alpha} \equiv v_{00}+\frac{\Delta}{2}\left\{1-\sqrt{ } 1+y^{2}\right\}+(d \phi / d R)^{2}
$$




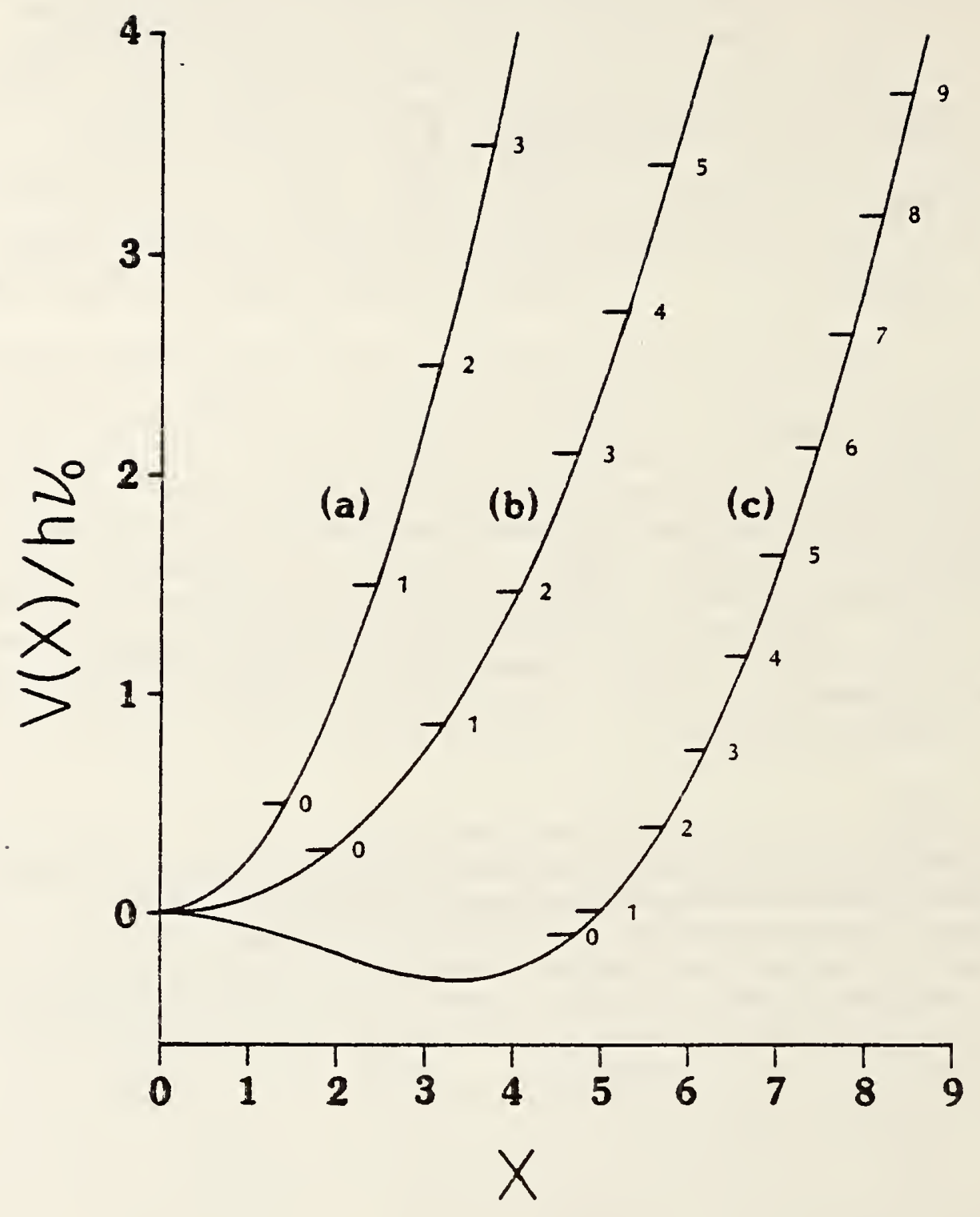

Fig, 4.3 curve (a) Pure harmonic potential in reduced units $V_{00}$ $=R^{2} / 4$ such that eigenvalues exist at $E_{n}=$ ( $n+$ $1 / 2)$.

curve (b) Adiabatic potential for perturbed $v_{10}$ mode of ${ }^{1} A "\left(S_{1}\right)$ state of propynal vibronically coupled to distant $\mathrm{A}^{\prime}\left(\mathrm{S}_{3}\right)$ state. In reduced units $\Delta=$ $25.3 i 3, \lambda=2.1197$ in Eq. (5).

curve (c) Adiabatic potential for perturbed $v_{4}$ mode of ${ }^{1} A_{2}\left(S_{1}\right)$ staţe of formaldehyde vibronically coupled to distant $B_{2}\left(S_{2}\right)$ state. In reduced units $\Delta=$ $24.920, \lambda=2.7890$ in Eq. (5). 
where,

$$
y=2 V_{01} / \Delta=\tan 2 \phi(R) .
$$

This potential is associated with the adiabatic wavefunction

$$
\left.E_{\alpha, n}\right\rangle=\left\{\cos \phi(R) \psi_{0}-\sin \phi(R) \psi_{1}\right\} \cdot \rho_{n}^{\alpha}\left(E_{\alpha, n}, R\right)
$$

where

$$
\left\{-\frac{\partial^{2}}{\partial R^{2}}+v_{\alpha \alpha}\right\} f_{n}^{\alpha}=E_{\alpha, n} f_{n}^{\alpha}
$$

The eigenvalues defined by Eq. (8) are shown in Fig. 4.3 and are found to reproduce the exact eigenvalues to a very high degree. Noticeable deviations $(= \pm 0.001)$ begin to occur at $\Xi \geqq 3.000$. However this accuracy is only attained if the adiabatic radial Born-Oppenheimer term $(\mathrm{d} \phi / d R)^{2}$ is retained in $E q$. (5). Actually this correction is only important for the lowest eigenvalues, and merely contributes about +0.008 to the $n=0$ eigenvalue for both systems.

Comparing Eq. (7) to Eq. (1) we see that the radial components of the exact two state wavefunction are approximated as follows,

$$
\begin{aligned}
& F_{n}=\cos (R) f_{n}^{\alpha}(R) \\
& G_{n}=-\sin (R) f_{n}^{\alpha}(R)
\end{aligned}
$$

These approximations have been tested numerically by evaluating the overlap of the exact and the adiabatic functions with the set of unperturbed harmonic functions in Eq. (4). The two sets of overlap integrals agree to within \pm 0.003 for $E_{n}<3.00$. This is more than adequate to insure that the adiabatic wavefunctions will reproduce any measurable vibronic intensity borrowing in the Propynal and Eormaldehyce spectra to within 5 percent.

5. Predissociation of the Schumann-Runge bands of $\mathrm{O}_{2}$ (P. S. Julienne)

The abscrption of solar ultraviolet radiation by the Schumann-Range bands of molecular oxygen control the transmission of the solar flux in the 200-175 $\mathrm{nm}$ range through the upper stratosphere and is 3 process of fundamental importance to stratospheric photochemistry.

Construction of an accurate solar transmission model has been hampered by experimental uncertainties in the oscillator strengths and predissociation linewidths of these bands. We have therefore constructed a theoretical model for the indivicual predissociation linewidths for the 
vibrational-rotational fine structure sublevels. This represents an extension of our earlier work on this problem (Julienne and Krauss, J. Mol. Spectrosc. 56, 270 (1975); Julienne, J. Mol. Spectrosc. 63, 50 $(1976))$. The main ingredient of this new work is to recalculate the spin-orbit coupling matrix elements between the predissociation $\mathrm{B}^{3} \Sigma_{\mathrm{u}}^{-}$ state and the ${ }^{1} \Pi u,{ }^{3} \Pi u,{ }^{5} \Pi u$, and $3 \Sigma_{u}^{+}$repulsive continuum states, since the original work required using an atomic approximation for the necessary matrix element. The new calculation gives very accurate spin-orbit matrix elements based on CAS-CI ab initio wavefunctions and the AESOP technique. The new spin-orbit matrix elements differ from the old ones by less than 25 percent. Thus, we obtain a quantitative improvement in line widths, but no major qualitative changes. This work was supported by NASA-Headquarters.

6. Nonadiabatic Line Broadening Calculations (P. S. Julienne)

A collaborative effort has been undertaken with $\mathrm{Dr}$. Linda Vahala, Old Dominion University, Norfolk, VA to use our nonadiabatic theory of atomic line broadening and our computer codes for scattering in a radiation field to calculate the distribution of pine structure states when sodium ${ }^{2} \mathrm{p}_{j}$ is excited by absorption in the far wings of the ${ }^{2} \mathrm{P}-{ }^{2} \mathrm{~S}$ resonance line perturbed by collisions with $\mathrm{He}, \mathrm{Ne}$, or Ar. The theoretical calculations were compared with experimental data by Dr. Mark Harvey, also at Old Dominion, and good agreement was found. Typical results are illustrated in Figure 4.4 .

7. Classical and Quantal Calculations of Radiative Decay Rates (P. S. Julienne)

This is a collaborative effort with Dr. Joel Tellinghuisen of Vanderbilt University. Dr. Tellinghuisen has discovered that the expectation value of

$$
A(R)=\frac{64 \pi^{4}}{3 h}\left[V^{\prime}(R)-V^{\prime \prime}(R)\right]^{3} \mu^{2}(R)
$$

for an upper state bound vibrational state is excellent approximation to the total spontaneous radiative decay rate for the state. Here $V$ ' and $V^{\prime \prime}$ represent the respective upper and lower molecular potentials and $\mu$ is the transition dipole. This result bears a strong relation to earlier work by ?. Julienne on collision-induced emission, for which a similar expression was utilized based on a classical path integral of the R-dependent spontaneous emission rate. We have shown that the theories of total emission rates for bound and free states are closely related, and both can be evaluated by classical, as well as quantal, expectation values. The approximate quantal and classical sum rule integrals were compared to the exact sums for several test cases involving bound levels and were found to be in excellent agreement with each other. Thus, molecular radiative lifetimes can be accurately evaluated in terms of single expectation values instead of difficult sums over all final states. A manuscript has been prepared for submission to the Journal of Chemical Physics. 


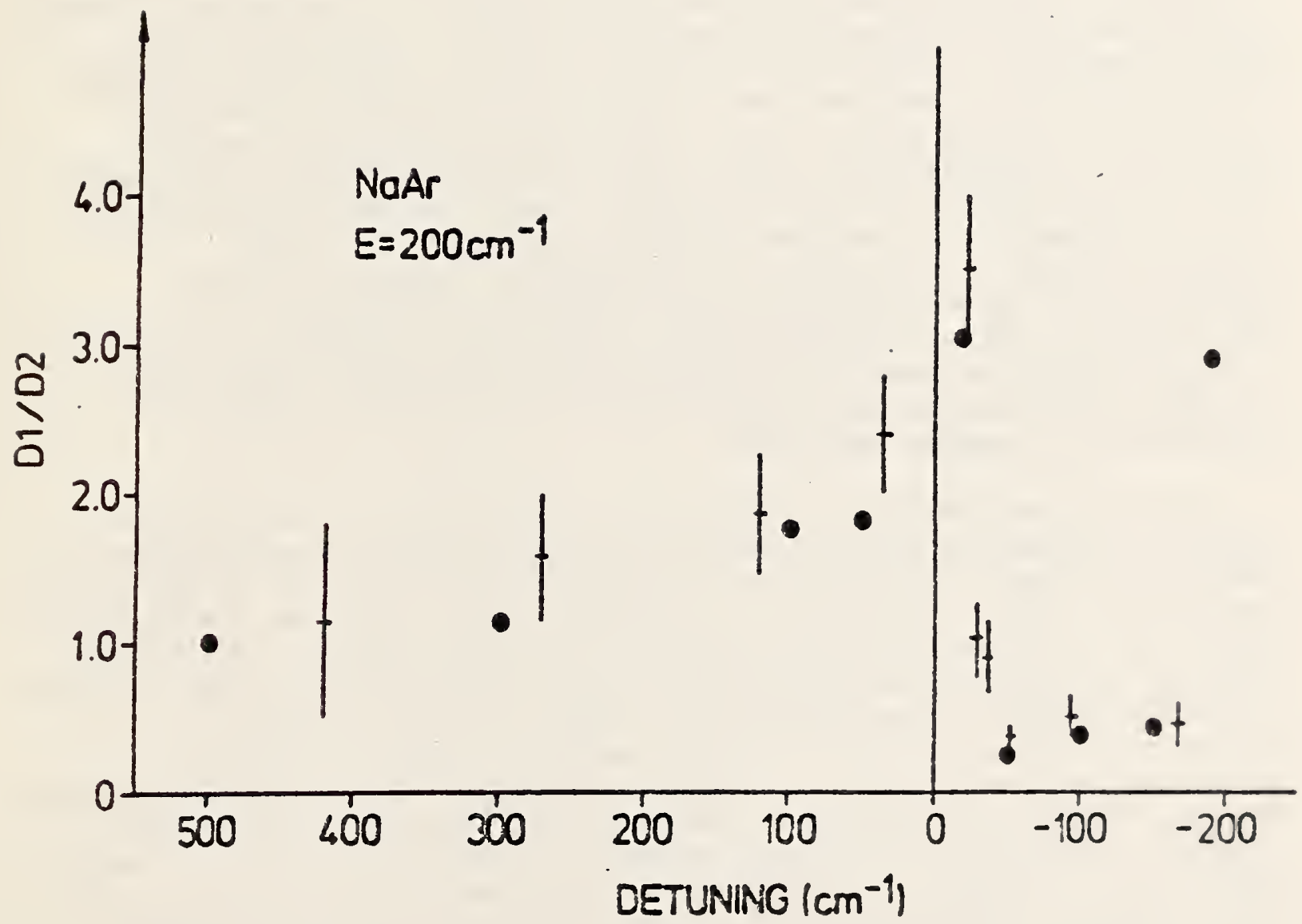

Fig. 4.4 Measured (+, error bars) and calculated ( $\cdot$, points) $\mathrm{Na}$ ${ }^{2} \mathrm{P}_{1 / 2}{ }^{2} \mathrm{P}_{3 / 2}$ Pluorescence branching ratios when $\mathrm{Na}$ is exclted by laser radiation during a collision with an argon perturber atom. The abscissa is the detuning irom the atcmic trans1tion frequency. 
In the development of microscopic theories of biochemical processes there is often a need for quantitative molecular data that are difficult or impossible to elucidate experimentally. Such data can be as simple as the strength of a bond between two interacting molecules or as complex as the nature of the transition state in an enzyme-catalyzed reaction. Interaction energies, conformational stabilities, potential energy surfaces, reaction coordinates, electronic charge distributions, and many other molecular properties may be determined theoretically using the computational methods of Quantum Chemistry. Recent advances in methodology, particularly in the algorithms for characterizing multidimensional potential energy surfaces, and the continued advancement of computer technology have allowed the application of accurate ab initio Quantum Chemistry methods to biochemical problems. Although direct quantum mechanical calculations are restricted to modest-sized fragments of three or four dozen atoms, more complex systems may be studied by hybrid methods which treat local sites quantum mechanically and the environment surrounding the sites, such as solvent molecules and distant molecular components, via semi-classical "reaction-field" Hamiltonians. It is also possible to use quantum mechanical calculations of fragment-fragment interactions to parameterize classical two-body potentials for use in more approximate "molecular mechanics" investigations of the secondary and tertiary structures and interactions of larger molecules such as polypeptide and poly-nucleic acids. Such potertials may also be used in Monte Carlo or molecular dynamic simulations of complex solutions.

The Quantum Chemistry Group has been developing new theories and computer programs which allow accurate quantum mechanical calculations on increasingly complex systems, including heavy metal atoms from anywhere in the periodic table. Our goal is to develop methods that are applicable to the study of interactions and reactions in large molecules, particularly proteins and enzymes containing metal atoms. To this end we will initiate a research program to develop hybrid "reaction-field" methods for determining the influence of solvents and surroundings on fragment structures and reactivities. We will also continue studying the influence of metal cations on polypeptide conformations, hydrogen bondings, and tautomer stabilities. In collabcration with other groups, we will begin generating a library of classicai atom-atom potentials for the interactions of polypeptides and residues with the inclusion of metal cation interactions. This will provide a database for future molecular dynamical and Monte Carlo simulations on systems of interest.

We will continue in our development of the multichannel quantum defect analysis (MCQDA) of molecular dissociation and explore its many possible applications in molecular spectroscopy and atomic scattering theory. In particular we shall exploit the half-collision analysis of non-adiabatic effects in dissociation continua as it applies to atomic lineshapes, photodissociation and predissociation. An important element of our research in the coming year will be extensive code development 
which will incorporate photodissociation and bound state capabilities into our close-coupled scattering programs. Our initial applications will be to diatonic systems, but we are approaching the code development wi th the intent of ultimately treating polyatomic moiecules, especially for loosely bound van der iaals and hydrogen-bonded systems. 


\section{PUBLICATIONS}

\section{(a) Publications of Past Year}

Aliev, M. R. and Hougen, J. T., "The Effects of Vibration-Rotation Interaction on the Quadrupole Hyperfine Structure of Molecular Rotational Levels," J. Mol. Spectrose. 106, 110 (1984).

Birnbaum, G., Frommhold, L., and Krauss, M., "Collision Induced Dipoles of Rare Gas Mixtures," J. Chem. Phys. 80, 2269 (1984).

Cavanagh, R. R. and King, D. S., Molecule-Surface Interactions and Dynamics,"J. Vac. Sci. Tech. A2, 1036 (1984).

Dang-Nhu, M., Pine, A. S., and Lafferty, W. J., "Les Intensities dans les Bandes $v_{5}, v_{7}$, et $v_{8}+v_{11}$ de 1 'Ethane ${ }^{12} \mathrm{C}_{2} \mathrm{H}_{6}$, "Can. J. Phys., 62, 512-519 (1984).

Ho, P.-L., Lee, C. H., Stephenson, J. C., and Cavanagh, R. R., "A Diamond Opto-electronic Switch," Optics Communication 46, 202 (1983).

Henry, L., Valentin, A., Lafferty, W. J., Hougen, J. T., Devi Malathy, V., Das,'P. P., and Rao Narahari, K., "Analysis of High Resolution Fourier Transform and Diode Laser Spectra of the $\nu_{9}$ Band of Ethane," J. Mol. Spectrose. 100, 260-289 (1983).

Hougen, J. T., "Some Effects of Spin-Orbit Interaction and Rotational Levels and Rotational Line Intensities in Vibrationally Unexcited ${ }_{A},{ }_{E}$ and ${ }^{2}$ Electronic States of $X Y_{4}$ Molecules," J. Mol. Spectrosc. $106,134-154(1984)$.

Jacox, M. E., "The Reaction of $F$ Atoms with Methyl Nitrite. Infrared Spectroscopic Evidence for the Stabilization of FON in an Argon Matrix," J. Phys. Chem. 87, 4950-4945 (1983).

Jacox, M. E., "Infrared Spectrum of the $\mathrm{C}_{2} F_{5}$ Free Radical Trapped in Solid Argon in Discharge Sampling Experiments," J. Phys. Chem. 88, 445-448 (1984).

Jacox, M. E., "Matrix Isolation Study of the Decomposition of $\mathrm{CF}_{3} \mathrm{NNCF}_{3}$ by Photons and by Excited Rare Gas Atom Bombardment of Energies Between 4.9 and $16.8 \mathrm{eV}, "$ Chem. Phys. 83, 171-180 (1984).

Jacox, M. E., "Photodecomposition of Nitromethane Trapped in Solid Argon," J. Phys. Chem. 88, 3373-3379 (1984).

Julienne, P. S. and Mies, F. H., "Nonadiabatic Theory of Atomic Line Broadening: Final State Distributions and the Polarization of Redistribution Radiation," Phys. Rev. A30, (1984). 
Knudtson, T. K. and Stephenson, J. C., "Vibrational Relaxation of HCl in Dilute $\mathrm{CCl}_{4}$ and $\mathrm{CCl}_{3} \mathrm{~F}$ Solutions," Chem. Phys. Letts. 107, 385 $(1984)$.

Krauss, M. and Stevens, W. J., "Effective Potentials in Molecular Quantum Chemistry," Ann. Rev. Phys. Chem. 35, 357 (1984).

Mak1, A. G. and Sams, R. L., "Diode Laser Spectra of Cis - HONO Near $850 \mathrm{~cm}^{-1}$ and trans-4ONO Near $1700 \mathrm{~cm}^{-1}$," J. Mol. Struct. 100, 215-221 (1983).

Maki, A. G., "Infrared Tunable Diode Laser Spectra of Lithium Fluoride at High Temperatures," J. Mol. Spect. 102, 361-367 (1983).

Mantell, D. A., Ryali, S. B., and Halier, G. L., "Time Resolved Infrared Emission Studies at $\mathrm{CO}_{2}$ Formed by $\mathrm{CO}$ Oxidation on $\mathrm{Pt}$ and $\mathrm{Pd}$, Chem. Phys. Lett. 102, 37 (1983).

Mies, F. H., "A Multichannel Quantum Defect Analysis of Diatomic Predissociation and Inelastic Atomic Scattering," J. Chem. Phys. 80, 2514-2525 (1984).

Mies, F. H. and Julienne, P. S., "A Multichannel Quantum Defect Analysis of Two-State Couplings in Diatomic Molecules," J. Chem. Phys. 80 , 2526-2536 (1984).

Nazemi, S., Javan, A., and Pine, A. S., "Collisional Effects on the Rovibrational Transitions of the HD Fundamental Band," J. Chem. Phys. 78, 4797-4805 (1983).

Ohashi, N. and Pine, A. S., "High Resolution Spectrum of the HCI Dimer," J. Chem. Phys., 8, $73 \cdot(1984)$.

0lson, W. B., "Minimization of Volume and Astigmatism in white Cells for use with Circular Sources and Apertures," Appi. Opt. 23, 1580 (1984).

Petersen, F. R., Wells, J.S., Siemsen, K. J., Robinson, A. M., and Maki, A. G., "Heterodyne Frequency Measurements and Analysis of $\mathrm{CO}_{2}$ Laser Hot Band Transitions," J. Mol. Spectrosc. 105, 324-330 (1984).

Pine, A. S., Coulombe, M. J., Camy-Peyret, C., and Flaud, J. M., "Atlas of the High Temperature Water Vapor Spectrum in the 3000 to $4000 \mathrm{~cm}^{-1}$ Region," J. Phys. Chem. Ref. Data, 12, 413-465 (1983).

Pine, A. G., Maki, A. G., Robiette, A. G., Krohn, 3. J., Watson, J. K. G., and Urbanek, Th.; "Tunable Laser Spectra of the Infrared-Active Fundamentals of Cubane," J. Am. Chem. Soc. 106, 391-897 (1984). 
Pollock, C. R., Petersen, F. R., Jennings, D. A., Wells, J. S., and Maki, A. G., "Absolute Frequency Measurements of the 2-0 Band of $C 0$ at 2.3um; Calibration Standard Frequencies from High Resolution Color Center Laser Spectroscopy," J. Mol. Spectrosc. 99, 357-368 (1983).

Rosasco, G. J., Hurst, W. S., and Lempert, W., "Three-beam Phase-modulation Technique for Coherent Raman Spectroscopy," Opt. Letters, Vol. 9, No. 1 (1983).

Singbeil, H. E. Gillis, Anderson, W. D., Davis Wellington, R., Gerry, M. C. L., Cohen, E. A., Pickett, H. M., Lovas, F. J., and Suenram, R: D:, "The Microwave and Millimeter-wave Spectra of Hypochlorous Acid;" J. Mol. Spectrose., 103, 466-485 (1984).

Stephenson, J. C., Blazy, J.A., and King, D.,S., "Spectroscopy and Collisional Quenching Rates for $\overline{\mathrm{A}} \mathrm{C}_{2} \mathrm{H}_{2}\left(\nabla_{3}=0,1,2\right)$, " Chem. Phys. 85,31 (1984).

Stevens, W. J. and Krauss, M., "Finite Field SCF Calculations of the Dipole Polarizabilities of Heavy Atoms Using Relativistic Effective Potentials," J. Phys. B16, 2921 (1983).

Suenram, R. D. and F. J. Lovas, "Millimeter Wave Spectrum of Chlorine Nitrate," J. Mol: Spectros., 105, 351-359 (1984).

Weber, A., Jennings, D. E., and Brault, J. W., "High Resolution Raman Spectroscopy of Gases with a Fourier Transform Spectrometer," Proc. IXth International Conference on Raman Spectroscopy, Tokyo 1984. 


\section{(b) Publications in Progress}

Basch, H., Stevens, W. J., and Krauss, M., "Electronic States of $\mathrm{Al}_{2}, "$ Chem: Phys. Lett. (in press).

Blazy, J. A., Stephenson, J. C., and King, D. S., "Infrared Multiphoton

Dissociation of Methyl Nitrite: Under Collisionless Conditions:

Product Yield and Energy Partitioning in the Primary Frangments," to be published in "Lasers as Reactants and Probes in Chemistry," William Jackson, Editor, (Howard University Press, 1984).

Cavanagh, R..R. and King, D. S., "Laser Studies of Surface Chemical Reactions," Chemical and Physics of Solid Surfaces, Vol. 5, (Springer-Verlag) (in press).

Heilweil, E. J., Casassa, M. P., Cavanagh, R. R., and Stephenson, J. C., "Picosecond Vibrational Energy Relaxation of Surface Hydroxyl Groups on Colloidal Silica," J. Chem. Phys. (in press).

Hougen, J. T. and Ohashi, N., "Group Theoretical Treatment of the Planar Internal Rotation Problem in $(\mathrm{HF})_{2}, " \mathrm{~J}$. Mol. Spectrosc. (in press).

Hougen, J. T., "Summary of Group Theoretical Results for Microwave and Infrared Studies of $\mathrm{H}_{2} \mathrm{O}_{2}, "$ Can. J. Phys. (in press).

Jacox, M. E., "Ground-State Vibrational Energy Levels of Polyatomic Transient Molecules ( 3 to 16 Atoms)," J. Phys. Chem. Ref. Data, (in press).

King, D. S. and Stephenson, J. C., "Kinetic Energy Disposal in the Unimolecular IRMPD of Methyl Nitrite," Chem. Phys. Lett. (submitted).

King, D. S. and Stephenson, J. C., "Infrared Multiphoton Dissociation of Methyl Nitrite in a Molecular Beam; Internal States of the Nitric Oxide Fragment," Chem. Phys. Letts. (submitted).

Petersen, F. R., Wells, J. S., Jennings, D. A., and Maki, A. C., "Improved Deuterium Bromide 1-0.Band Molecular Constants from Heterodyne Frequency Measurements," J. Mol. Spectrosc. (in press).

Pine, A. S., Lafferty, W. J., and Howard, B. J., "Vibrational Predissociation, Tunneling and Rotational Saturation in the HF and DE Dimers," J. Chem. Phys. (in press).

Pine, A. S., "Tunable Laser Spectroscopy of Upper Atmospheric Molecules," CMA "Norkshop, edited by A. Weber.

Pine, A. S., Fried, A., and Elkins, J. N., "Spectral Intensities in the Fundamental Bands of $\mathrm{HF}$ and $\mathrm{HCl}, " J$. Mol. Spectrosc. (submitted). 
Pollock, C. P., Petersen, F. R., Jennings, D. E., Wells, J. S., and Maki, A. G., "Absolute Frequency Measurements of the $00^{\circ} 2-00^{\circ} 0$, 2001-00॰ Bands of $\mathrm{N}_{2} \mathrm{O}$ by Heterodyne Spectroscopy," J. Mol. Spectrosc. (in press).

Rumble, J. R., Stevens, W. J., and Truhlar, D. G., "Effect of Electron Correlation in the Target Wavefunction on Electron-Molecule Scattering," J. Phys. B (in press).

Stevens, W. J., Basch, H., and Krauss, M., "Compact Effective Core Potentials and Efficient Shared-Exponent Basis Sets for the First- and Second-Row Atoms, Li-Ar," J. Chem. Phys. (submitted). 
(C) Recent Publications of New Staff Members: (Casassa, Heilweil, Mantell, Perry, and Woodward) Describing Work in Previous Positions

Casassa, M. P., Western, C. M., Celii, F. G., Brinza, D. E., and Janda, K. C., "Infrared Photodissociation of Hydrogen-bonded Clusters: $\mathrm{C}_{2} \mathrm{H}_{4} \cdot \mathrm{HF}$ and $\mathrm{C}_{2} \mathrm{H}_{4} \cdot \mathrm{HCl}, " \mathrm{~J}$. Chem. Phys. 79, 3227 (1983).

Casassa, M. P., Western, C. M., and Janda, K. C., "Effects of Inhomogeneity in the Infrared Photodissociation Spectra of $\left(\mathrm{C}_{2} \mathrm{H}_{4}\right)_{2}$, $\mathrm{C}_{2} \mathrm{H}_{4} \cdot \mathrm{HF}$ and $\mathrm{C}_{2} \mathrm{H}_{4} \cdot \mathrm{HCl}, " \mathrm{~J}$. Chem. Phys. (in press).

Celi1, F. G., Casassa, M. P., and Janda, K. C., "Photodesorption of Weakly'Bound Molecules," Surface Science'(submitted).

Chupka, W. A., Woodward, A. M., and Colson, S. D., "Electron Photodetachment from Transient Negative Ions in the Multiphoton Ionization of $\mathrm{CH}_{3} \mathrm{I}, " \mathrm{~J}$. Chem. Phys. (in press).

Doany, F. E., Heilweil, E. J., Moore, R., and Hochstrasser, R. M., "Picosecond Study of an Intermediate in the Trans to Cis Isomerization Pathway of Stiff Stilbene," J. Chem. Phys. 80, 201 (1984).

Mantell, D. A., Ryali, S. B., Haller, G. L., and Fenn, J. B., "The Dynamics of CO Oxidation of Pt Deduced from Translational, Rotational and Vibrational Excitation in the Product $\mathrm{CO}_{2}$," Surface Science (submitted).

Moll, D. J., Perry, J. W., Kuppermann, A., and Zewail, A. H., "High-Energy Overtone Photoacoustic Spectroscopy of Some Deuterated Methanes I. Rotational Analysis," J. Chem. Phys., (submitted).

Perry, J. W., Scherer, N. F., and Zewail, A. H., "Picosecond Pump-Probe Multiphoton Ionization of Isolated Molecules: IVR and Coherence," Chem. Phys. Lett. 103, 1 (1983).

Perry, J. W. and Zewail, A. H., "High-Energy CH-overtone Spectra of Benzene at $1.8 \mathrm{~K}, " \mathrm{~J}$. Chem. Phys. 80, 5333 (1984).

Perry, J. W., Moll, D. J., Zewail, A. H., and Klippermann, A., "High Energy Overtone Photoacoustic Spectroscopy of Some Deuterated Methanes II. Vibratinal Analysis," J. Chem. Phys., (submitted).

Western, C. M., Casassa, M. P., and Janda, K. C., "Infrared Photodissociation of the Hindered Internal hotors $\mathrm{Ne} \cdot \mathrm{C}_{2} \mathrm{H}_{4}$ and $\mathrm{Ar} \cdot \mathrm{C}_{2} \mathrm{H}_{4}, "$ J. Chem. Phys. 80, 4781 (1984).

Woodward, A. M., Chupka, W. A., and Colson, S. D., "T'wo-Color Laser Studies of the Multiphoton Ionization and Dissociation of trans 1,3-Butadience," J. Phys. Chem. (in press). 



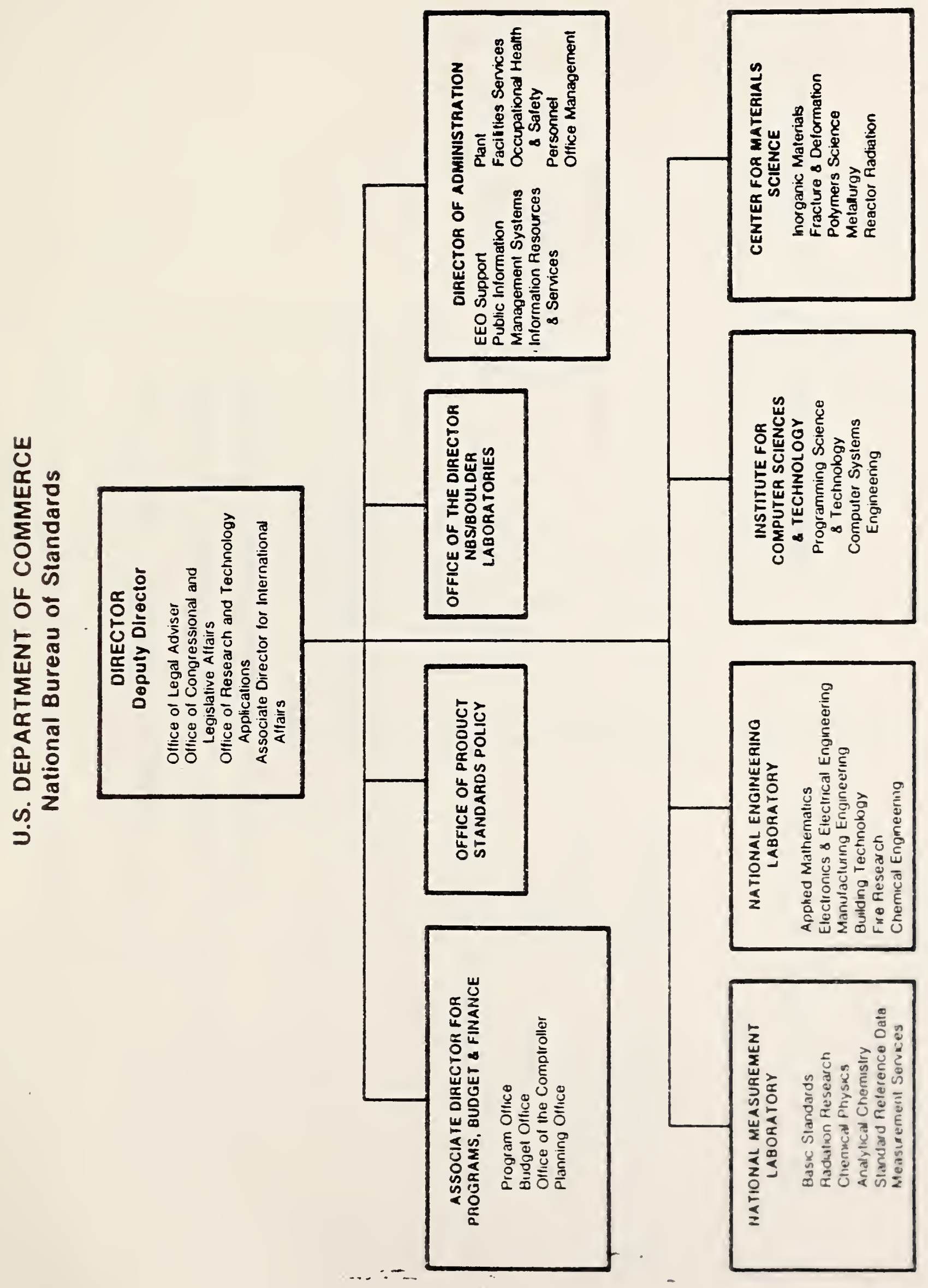





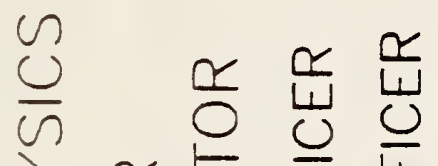

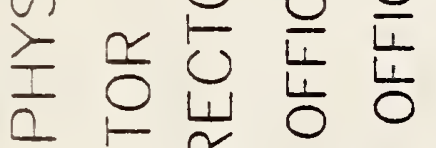

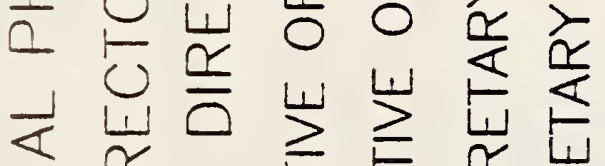

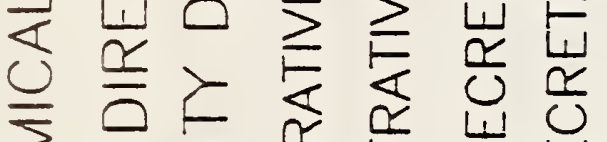

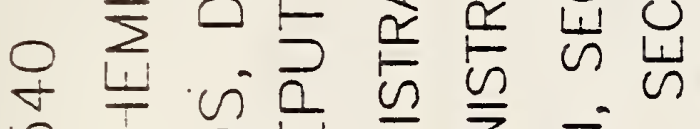

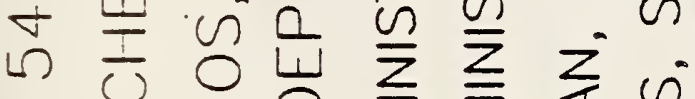

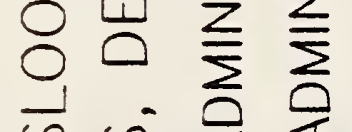

吕的食定出

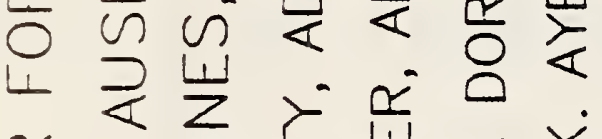

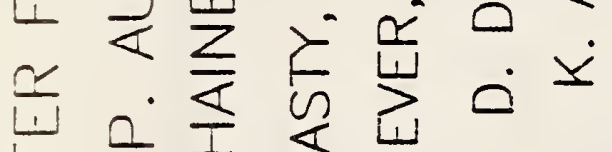

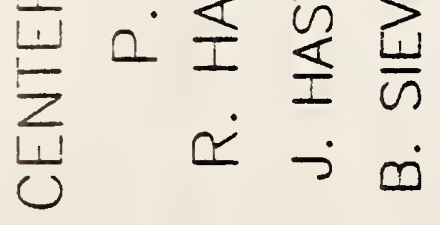

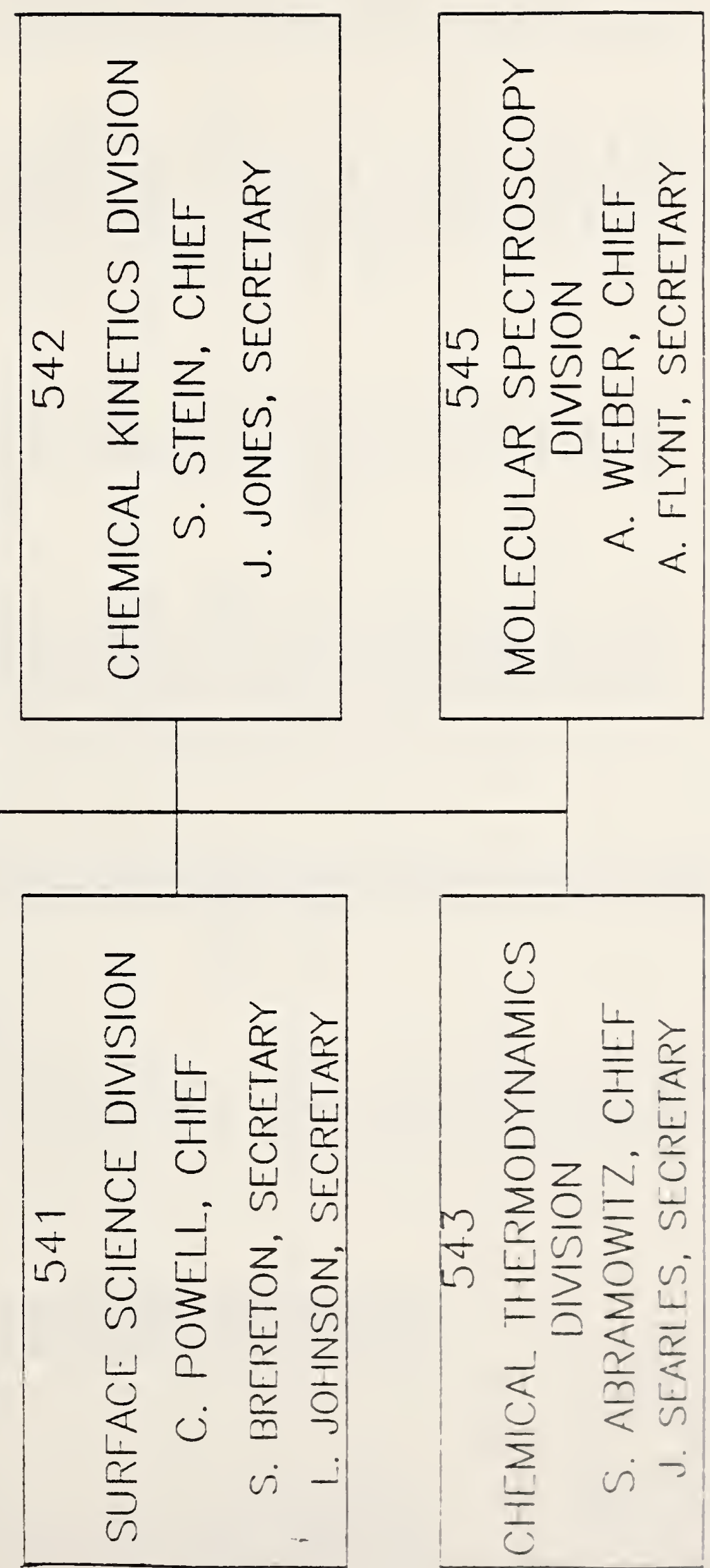



NBS-114A (REV. 2-9C)

U.S. OEPT. OF COMM.

BIBLIOGRAPHIC DATA

SHEET (See instructions) 1. PUBLICATION OR
REPORT NO.

NBSIR $84-2982$
2. Performing Organ. Report Nof 3. Publication Dase

October, 1984

4. TITLE AND SUBTITLE

ANNUAL REPORT - CENTER FOR CHEMICAI PEYSICS

5. $A U T H O R(S)$

P. J. Ausloos and R. A. Faines

6. PERFORMING ORGANIZATION (If joint or other thon NBS, see instructions)

7. Contract/Grant No.

MATIOMAL BUREAU OF STANDARDS

DEPARTHENT OF COMMERCE

WASHINGTON. D.C. 20234

8. Type of Report \& Period Covered

9. SPONSORING ORGANIZATION NAME AND COMPLETE ADDRESS (Street, City, SlOte, ZIP)

10. SUPPLEMENTARY NOTES

Document describes a computer orogram: SF-185. FIPS Software Summary, is attached.

11. ABSTRACT (A 200-word or less fociual summory of most significont information. If document includes o significont bibliography or literature survey. mention it here)

This report summarizes research projects, neasurement method derelcpment, testing and data evaluation activities carried out during Fiscal Year 1984 in the NBS Center for Chemical Physics. These actitities fall in the areas of surface science, chemical kinetics, chemical thermodynamics and molecular spectroscopy.

12. KEY INOROS (Six to twelve entries; alphabetical order: capitolize only prooer names; ond separate key words ty semicolons) Chemical kinetics; chemicaI Jhysics; nolecular spectroscopy; surtace science; themodinamics-

13. AVAILABIL!TY

Unlimited

For Official Distribution. Co Not Release to NTIS

Order From Superıntencent of Documents, U.S. Government Prining Office, Wasnington. D.C. 20402.

E Order From National Technical Information Service (NTIS), Springrield, VA. Z2!6l
14. NO. OF PRINTED PAGES 255

15. Price 22. 00 


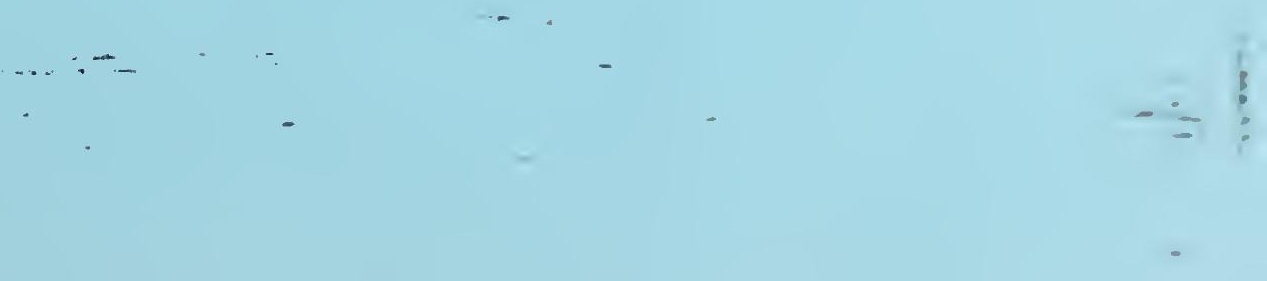


